\title{
Catalytic, Enantioselective Syn-Oxyamination of Alkenes
}

\author{
Emily M. Mumford,${ }^{\dagger}$ Brett N. Hemric, ${ }^{\dagger}$ and Scott E. Denmark* \\ Department of Chemistry, University of Illinois at Urbana-Champaign, Urbana, Illinois 61801

\section{SUPPORTING INFORMATION}

TABLE OF CONTENTS

PAGE

General Experimental

$S 2$

Literature Preparations

S5

Experimental Procedures

$\underline{\text { Preparation of Substrates }}$

General Procedure I: Synthesis for 1,2-Oxyamination of Alkenes

S11

$\underline{\text { Survey of } \mathrm{N} \text {-Sulfonamide Benzamides (Table 2) }}$

$S 12$

$\underline{\text { Survey of Chiral Diselenide Catalysts (Table 3) }}$

$S 14$

Determination of Absolute Configuration for Oxazoline Products

$S 15$

Effect for $\mathrm{NaF}$ on the Alkene 1,2-Oxyamination Reaction

$S 15$

$\underline{\text { General Procedure for Catalytic Oxyamination }}$

$S 17$

$\underline{\text { Substrates Displaying Poor Conversion to the Desired Oxazoline }}$

S79

$\underline{\text { Reaction Kinetics Comparison for trans-4-Octene (Figure 3) }}$

581

$\underline{\text { References }}$

583

NMR Spectra and CSP-HPLC/SFC Traces

$S 85$ 


\section{General Experimental}

General Procedures: Glassware and stir bars were either flame-dried or dried in an oven at $60{ }^{\circ} \mathrm{C}$ overnight. Optimization and small-scale screens were performed in 1 Dram glass vials with Teflon-coated micro stir bar. Descriptive runs were conducted in $20 \mathrm{~mL}$ scintillation vials with Teflon-coated stir bar. All other reactions were performed in round-bottom flasks with rubber septa and Teflon-coated stir bars, unless otherwise noted. Plastic syringes were used for the transfer of pure solvents, while glass pipets were used for transfer of crude reaction solutions.

NMR Spectroscopy: ${ }^{1} \mathrm{H}$ and ${ }^{13} \mathrm{C}\left[{ }^{1} \mathrm{H}\right]$ Nuclear Magnetic Resonance spectra were recorded on a Bruker $500 \mathrm{MHz}$ spectrometer $\left(500 \mathrm{MHz},{ }^{1} \mathrm{H} ; 126 \mathrm{MHz},{ }^{13} \mathrm{C}\right)$ at $21{ }^{\circ} \mathrm{C}$ unless otherwise noted. Chemical shifts are reported in parts per million and referenced to residua protium in $\mathrm{CDCl}_{3}(\delta=$ $\left.7.26 \mathrm{ppm},{ }^{1} \mathrm{H} ; 77.16 \mathrm{ppm},{ }^{13} \mathrm{C}\left[{ }^{1} \mathrm{H}\right]\right) .{ }^{19} \mathrm{~F}$ and ${ }^{31} \mathrm{P}$ Nuclear Magnetic Resonance spectra were recorded on a Varian $500 \mathrm{MHz}$ spectrometer $\left(471 \mathrm{MHz},{ }^{19} \mathrm{~F} ; 161.97 \mathrm{MHz},{ }^{31} \mathrm{P}\right)$ at $21{ }^{\circ} \mathrm{C}$ unless otherwise noted. Chemical shifts are reported in parts per million and referenced to hexafluorobenzene $\left(\mathrm{C}_{6} \mathrm{~F}_{6}\right)$ and triphenylphosphine oxide $\left(\mathrm{Ph}_{3} \mathrm{PO}\right)$, respectively in $\mathrm{CDCl}_{3}(\delta=-$ $\left.161.64 \mathrm{ppm},{ }^{19} \mathrm{~F} ; 29.45 \mathrm{ppm},{ }^{31} \mathrm{P}\right)$. NMR values are reported as follows: chemical shift, multiplicity $(\mathrm{s}=$ singlet, $\mathrm{d}=$ doublet, $\mathrm{t}=$ triplet, $\mathrm{q}=$ quartet, $\mathrm{pent}=$ pentet, $\mathrm{m}=$ multiplet $)$, coupling constant (Hz), integration, and signal assignment. ${ }^{1} \mathrm{H}$ and ${ }^{13} \mathrm{C}$ assignments are corroborated through 2-D NMR experiments (COSY, HSQC, HMBC).

Infrared Spectroscopy: Infrared (IR) spectra were recorded on a Perkin-Elmer FTIRATR system as thin films. Peaks are reported in $\mathrm{cm}^{-1}$ with indicated relative intensities: $\mathrm{s}$ (strong, 0-33\% T); m (medium, 34-66\% T), w (weak, 67-100\% T), and br (broad).

Mass Spectrometry: Mass spectrometry (MS) was performed by the University of Illinois Mass Spectrometry Laboratory. Electron Impact (EI+) spectra were performed at $70 \mathrm{eV}$ using 
methane as the carrier gas, with either a double focusing sector field (DFSF) or time-of flight (TOF) mass analyzer. Chemical Ionization $(\mathrm{CI}+)$ spectra were performed with methane reagent gas, with either a double focusing sector field (DFSF) or time-of-flight (TOF) mass analyzer. Electrospray Ionization (ESI+) spectra were performed using a time-of-flight (TOF) mass analyzer. Data are reported in the form of $\mathrm{m} / \mathrm{z}$ (intensity relative to the base peak $=100$ ).

Melting Points: Melting points (mp) were determined on a Thomas-Hoover capillary melting point apparatus in vacuum-sealed capillary tubes and are corrected.

Elemental Analysis: Elemental analysis was performed by the University of Illinois Microanalysis Laboratory. Reported data is the average of at least 2 runs.

Distillation: Bulb-to-bulb distillation was performed on a Kugelrohr, with boiling points (b.p.) corresponding to uncorrected air-bath temperatures (ABT). A vacuum of $10^{-5} \mathrm{mmHg}$ was achieved using a BOC Edwards SI100 diffusion pump.

Chromatography: Analytical thin-layer chromatography was performed on Merck silica gel 60 F254 plates. TLC plates were visualized by exposure to ultraviolet light and treatment with either vanillin or $\mathrm{KMnO}_{4}$ stain. Retention factor $\left(\mathrm{R}_{\mathrm{f}}\right)$ values reported were measured using a $10 \times$ $2 \mathrm{~cm}$ TLC plate in a developing chamber containing the solvent system described. Flash column chromatography was performed using Silicycle SiliaFlash ${ }^{\circledR} \mathrm{P} 60$ (40-63 $\mu \mathrm{m}$ particle size, $230-400$ mesh) $\left(\mathrm{SiO}_{2}\right)$. Unless otherwise specified, "silica" refers to P60 grade silica gel. Analytical chiral stationary phase high pressure liquid chromatography (CSP-HPLC) was performed with a UV detector (220 nm) using Supelco Astec Cellulose, Regis $R, R$-Whelk-O1, Daicel Chiralcel OJ-H, and Daicel Chiralpak IB-3 columns. Analytical chiral stationary phase supercritical fluid chromatography (CSP-SFC) was performed with a UV detector (220 nm) using Chiralpak AD-H column. 
Solvents: Reaction solvents tetrahydrofuran (THF) (Fisher, HPLC grade), ether $\left(\mathrm{Et}_{2} \mathrm{O}\right)$ (Fisher, BHT stabilized ACS grade), and dichloromethane $\left(\mathrm{CH}_{2} \mathrm{Cl}_{2}\right)$ (Fisher, unstabilized HPLC grade) were dried by percolation through two columns packed with neutral alumina under a positive pressure of argon. Reaction solvent toluene (ACS grade) was dried by percolation through a column packed with neutral alumina and a column packed with Q5 reactant (supported copper catalyst for scavenging oxygen) under a positive pressure of argon. Reaction solvent dimethylformamide (DMF) (Fischer, ACS grade) was dried by percolation through two columns of activated molecular sieves. Reaction solvent acetonitrile $\left(\mathrm{CH}_{3} \mathrm{CN}\right)$ (Fisher, amylene stabilized, ACS grade) was distilled from $\mathrm{CaH}_{2}$. Reaction solvent 1,2-dichloroethane $\left(\mathrm{ClCH}_{2} \mathrm{CH}_{2} \mathrm{Cl}\right)$ (Millipore Sigma, ACS grade) was dried over $3 \AA$ molecular sieves for 1 day prior to use. Reaction solvent ethanol (absolute, Decon Laboratories) was used as received. Solvents for filtration, transfers, chromatography, and recrystallization were benzene (ACS grade), dichloromethane $\left(\mathrm{CH}_{2} \mathrm{Cl}_{2}\right)$ (amylene stabilized, ACS grade), ether $\left(\mathrm{Et}_{2} \mathrm{O}\right)$ (BHT stabilized, ACS grade), ethyl acetate (EtOAc) (ACS grade), hexane (HPLC grade), ethanol (EtOH) (ACS grade), methanol $(\mathrm{MeOH})\left(\mathrm{ACS}\right.$ grade), pentane (ACS grade), and petroleum ether $\left(35-60^{\circ} \mathrm{C}, \mathrm{ACS}\right.$ grade).

Quantitative ${ }^{1} \mathbf{H}$ NMR: To a solution of the compound in question (9.8-13.9 mg, 0.028$0.037 \mathrm{mmol})$ in $\mathrm{CDCl}_{3}(500 \mu \mathrm{L})$ was added 1,1,2,2-tetrachloroethane (3.4-6.2 mg, 0.020-0.036 mmol). The ${ }^{1} \mathrm{H}$ NMR was obtained using 16 scans with a d1 (relaxation delay) of 30 seconds. The integration value of any non-overlapping $(1 \mathrm{H}$, single proton) signal of the compound in question (typically in the 6.0-4.0 ppm region) was integrated and normalized to 1.00 . The ${ }^{1} \mathrm{H}$ signal of the internal standard (singlet, $5.95 \mathrm{ppm}, 2 \mathrm{H}$ ) was integrated. These values were used in the following equations, following the guidelines in Organic Synthesis' "instructions for authors" (http://www.orgsyn.org/instructions.aspx). 


$$
\begin{gathered}
\text { Molar ratio }=\frac{\left[\frac{\text { integral of cmpd signal }}{\# \text { of protons in cmpd signal }]}\right.}{\left[\frac{\text { integral of std signal }}{\# \text { of protons in std signal }}\right]} \\
\text { purity of cmpd }=\frac{(m g \text { of std })(M W \text { of cmpd })(\text { Molar ratio })}{(m g \text { of cmpd })(M W \text { of } \text { std })}
\end{gathered}
$$

Commercial Reagents: Diphenyl diselenide $\left(\mathrm{Ph}_{2} \mathrm{Se}_{2}\right)$ (TCI, 97\%), trans- $\beta$-methylstyrene (2a, TCI, 97\%), (E)-cinnamyl chloride (2f, Alfa-Aesar, 95\%), (E)-4-octene (2k, GFS, 96\%), transanethole (2m, Millipore Sigma, 99\%), \%), trans-stilbene (2r, Millipore Sigma, 96\%), sodium borohydride (Millipore Sigma, 98\%), di-tert-butyl decarbonate (Oakwood), and N,N'dimethylethylenediamine (Millipore Sigma, 98\%) were used as received. 1-Fluoro-2,4,6trimethylpyridinium tetrafluoroborate $\left(2,4,6-\mathrm{Me}_{3} \mathrm{PyF}^{+} \mathrm{BF} 4^{-}\right)(\mathrm{TCI}$ or Millipore Sigma, 95\%) and cesium carbonate (Alfa Aesar, 99\%) were stored in the glove box and used as received. Methyl trifluoromethanesulfonate (Millipore Sigma, 98\%) and diphenylphosphine (Millipore Sigma, 98\%) were transferred to Schlenk flasks under argon and used as received. Diisobutylaluminum hydride solution (Millipore Sigma, 1.0 $\mathrm{M}$ in hexanes) was stored at $-20^{\circ} \mathrm{C}$ and used as received. Copper(I) iodide was purified as previously described. ${ }^{1}$

\section{Literature Preparations}

The following compounds were synthesized according to previously reported literature preparations: (1S,1'S)-Diselanediylbis(7-methoxy-1,2,3,4-tetrahydronaphthalene-8,1-diyl)bis(2naphthoate) (3a), ${ }^{2}$ 2-methyl- $N$-tosylbenzamide (1f), ${ }^{3}$ 2-bromo- $N$-tosylbenzamide $(\mathbf{1 k}),{ }^{4}$ 2-fluoro$N$-tosylbenzamide (11), ${ }^{5} \quad N$-cinnamyl-4-methyl- $N$-phenylbenzenesulfonamide $\quad(\mathbf{2 c}),{ }^{6} \quad 2$ cinnamylisoindoline-1,3-dione (2d) ${ }^{7}$ cinnamyl benzyl ether $(\mathbf{2 g}),{ }^{8}(E)-3$-(3-benzyloxy)prop-1-en1-yl)thiophene $\quad(\mathbf{2 o}),{ }^{9} \quad(E)-2-(3-(b e n z y l o x y) p r o p-1-e n-1-y l) n a p h t h a l e n e \quad(2 \mathbf{p}),{ }^{2} \quad(E)-1-(3-$ (benzyloxy)prop-1-en-1-yl)naphthalene $(\mathbf{2 q}),{ }^{10}$ and (E)-1,2-bis(3-methoxyphenyl)ethene (2s). ${ }^{11}$ 
The authors would like to thank Dr. Zhonglin Tao and Dr. Bradley Gilbert for the preparation of the following substrates: (E)-(3-methylbut-1-en-1-yl)benzene (2b), ${ }^{12}$ (E)-2-(4phenylbut-3-en-1-yl)isoindoline-1,3-dione $(\mathbf{2 e}),{ }^{13}$ methyl $(E)-4$-phenylbut-3-enoate $(\mathbf{2 h}),{ }^{14}(E)-2$ methyl-2-(4-phenylbut-3-en-1-yl)-1,3-dioxolane (2i), (E)-1-bromo-2-(prop-1-en-1-yl)benzene (2n), ${ }^{15}$ and (E)-1-methoxy-4-(4-(trifluoromethyl)styryl)benzene (2t) ${ }^{2}$ 


\section{Experimental Procedures}

\section{Preparation of Substrates}

\section{Preparation of (E)-2-Methyl-2-(4-phenylbut-3-en-1-yl)-1,3-dioxolane (2i)}

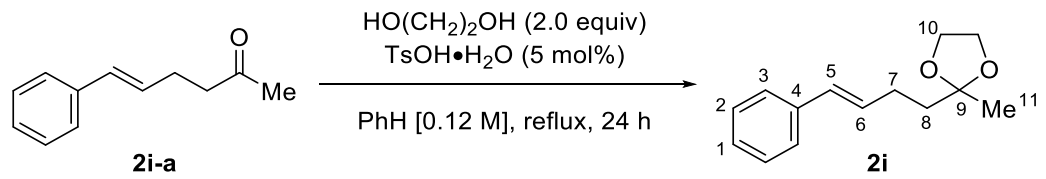

To a solution of $(E)-6$-phenylhex-5-en-2-one $(\mathbf{2 i - a})^{16}(1.22 \mathrm{~g}, 7.0 \mathrm{mmol})$ and ethylene glycol $(0.87 \mathrm{~g}, 14.0 \mathrm{mmol}, 2.0$ equiv $)$ in benzene $(60 \mathrm{~mL})$ in a $100 \mathrm{~mL}$ round-bottom flask under argon with an appended Dean-Stark apparatus was added $p$-toluenesulfonic acid monohydrate (66.6 mg, 0.05 equiv, $0.35 \mathrm{mmol}$ ). The mixture was heated at reflux for $24 \mathrm{~h}$ then cooled to $21{ }^{\circ} \mathrm{C}$ and concentrated under reduced pressure by rotary evaporation $\left(30{ }^{\circ} \mathrm{C}, \sim 20 \mathrm{~mm} \mathrm{Hg}\right)$. Column chromatography (100 g silica gel, $5.5 \mathrm{~cm}$ column) eluting with hexanes/ethyl acetate, 98:2 (15-mL fractions) yielded $1.53 \mathrm{~g}(100 \%)$ of $\mathbf{2} \mathbf{i}$ as a clear, slightly yellow oil. Spectral data was consistent with previous literature report. ${ }^{17}$

\section{Data for 2i:}

${ }^{1} \mathrm{H}$ NMR: $\quad\left(500 \mathrm{MHz}, \mathrm{CDCl}_{3}\right)$

$\delta 7.34(\mathrm{~d}, J=7.4 \mathrm{~Hz}, 2 \mathrm{H}, \mathrm{HC}(3)), 7.29$ (t, $J=7.7 \mathrm{~Hz}, 2 \mathrm{H}, \mathrm{HC}(2)), 7.19$ (t, $J=$ $7.2 \mathrm{~Hz}, 1 \mathrm{H}, \mathrm{HC}(1)), 6.41$ (d, $J=15.9 \mathrm{~Hz}, 1 \mathrm{H}, \mathrm{HC}(5)), 6.24$ (dt, $J=15.7,6.8$ $\mathrm{Hz}, 1 \mathrm{H}, \mathrm{HC}(6)), 4.00-3.93$ (m, $\left.4 \mathrm{H}, \mathrm{H}_{2} \mathrm{C}(10)\right), 2.33$ (dtd, $J=8.4,6.7,1.5 \mathrm{~Hz}, 2$ $\left.\mathrm{H}, \mathrm{H}_{2} \mathrm{C}(7)\right), 1.86-1.79\left(\mathrm{~m}, 2 \mathrm{H}, \mathrm{H}_{2} \mathrm{C}(8)\right), 1.37$ (s, $\left.3 \mathrm{H}, \mathrm{H}_{3} \mathrm{C}(11)\right)$.

${ }^{13} \mathrm{C} \mathrm{NMR:} \quad\left(126 \mathrm{MHz}, \mathrm{CDCl}_{3}\right)$

$\delta 137.8(\mathrm{C}(4)), 130.5(\mathrm{C}(6)), 129.7(\mathrm{C}(5)), 128.4(\mathrm{C}(2)), 126.8(\mathrm{C}(1)), 125.9$ (C(3)), $109.7(\mathrm{C}(9)), 64.7(\mathrm{C}(10)), 38.7(\mathrm{C}(8)), 27.6(\mathrm{C}(7)), 24.0(\mathrm{C}(11))$. 


\section{IR: (thin-film)}

$3025(w), 2982(w), 2948(w), 2879(w), 1651(w), 1598(w), 1577(w), 1496$

(w), 1447 (w), $1375(\mathrm{~m}), 1218(\mathrm{~m}), 1134(\mathrm{~m}), 1114(\mathrm{~m}), 1057(\mathrm{~s}), 964(\mathrm{~m}), 948$

(m), $866(\mathrm{~m}), 825(\mathrm{w}), 787(\mathrm{w}), 743(\mathrm{~m}), 693(\mathrm{~s}), 620(\mathrm{w}), 568(\mathrm{w}), 535(\mathrm{w})$

HRMS: $\quad\left(\mathrm{ESI}^{+}\right)$Cald for $\mathrm{C}_{14} \mathrm{H}_{19} \mathrm{O}_{2}{ }^{+}: 219.1385$, found: 219.1390 .

\section{Preparation of (E)-1-(Prop-1-en-yl)-4-(trifluoromethyl)benzene (2l)}

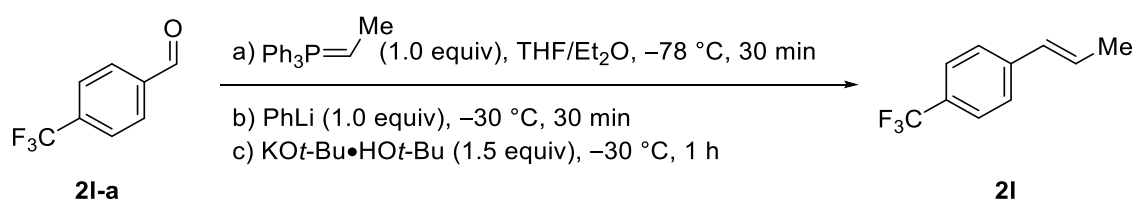

To an anhydrous solution of ethyl triphenylphosphonium bromide (3.79 g, $10.2 \mathrm{mmol}, 1.02$ equiv) in tetrahydrofuran $(22.0 \mathrm{~mL})$ and diethyl ether $(11.0 \mathrm{~mL})$ at $21^{\circ} \mathrm{C}$ was added phenyllithium (5.56 mL, $1.8 \mathrm{M}$ in hexanes, $10.0 \mathrm{mmol}$ ). The resulting solution was stirred at $21^{\circ} \mathrm{C}$ for $30 \mathrm{~min}$, then was cooled in a $-78{ }^{\circ} \mathrm{C}$ dry ice/iso-propanol bath. 4-(Trifluoromethyl)benzaldehyde (1.37 $\mathrm{mL}, 10.0 \mathrm{mmol}, 1.0$ equiv) was added dropwise and the mixture was stirred at $-78{ }^{\circ} \mathrm{C}$ for $30 \mathrm{~min}$. Phenyllithium (5.56 mL, 1.8 M in hexanes, $10.0 \mathrm{mmol})$ was added and the reaction warmed to $30{ }^{\circ} \mathrm{C}$ and was then stirred at $-30^{\circ} \mathrm{C}$ for $30 \mathrm{~min}$. A complex of potassium tert-butoxide/tert-butanol ( $2.80 \mathrm{~g}, 15.0 \mathrm{mmol}, 1.5$ equiv) was added and the mixture was stirred at $-30{ }^{\circ} \mathrm{C}$ for $1 \mathrm{~h}$, then 21 ${ }^{\circ} \mathrm{C}$ for $30 \mathrm{~min}$. The mixture was diluted with water $(50.0 \mathrm{~mL})$, transferred to a $250-\mathrm{mL}$ separatory funnel and extracted with ethyl acetate $(100 \mathrm{~mL} \mathrm{X} \mathrm{3).} \mathrm{The} \mathrm{combined} \mathrm{organic} \mathrm{layers} \mathrm{were} \mathrm{washed}$ with brine $(50 \mathrm{~mL})$, dried over magnesium sulfate $(5 \mathrm{~g})$, filtered, and concentrated under reduced pressure by rotary evaporation $\left(30^{\circ} \mathrm{C}, \sim 20 \mathrm{~mm} \mathrm{Hg}\right)$. Column chromatography (50 g silica gel, 4 $\mathrm{cm}$ column) eluting with hexanes (50-mL fractions) yielded $1.35 \mathrm{~g}(72 \%)$ of $2 \mathrm{I}$ as a clear oil. Spectral data was consistent with previous reports. ${ }^{18}$ 


\section{Preparation of \pm trans-5-((Benzyloxy)methyl)-4-phenyl-2-(o-tolyl)-4,5-dihydrooxazole $( \pm 4 \mathrm{~g})$}
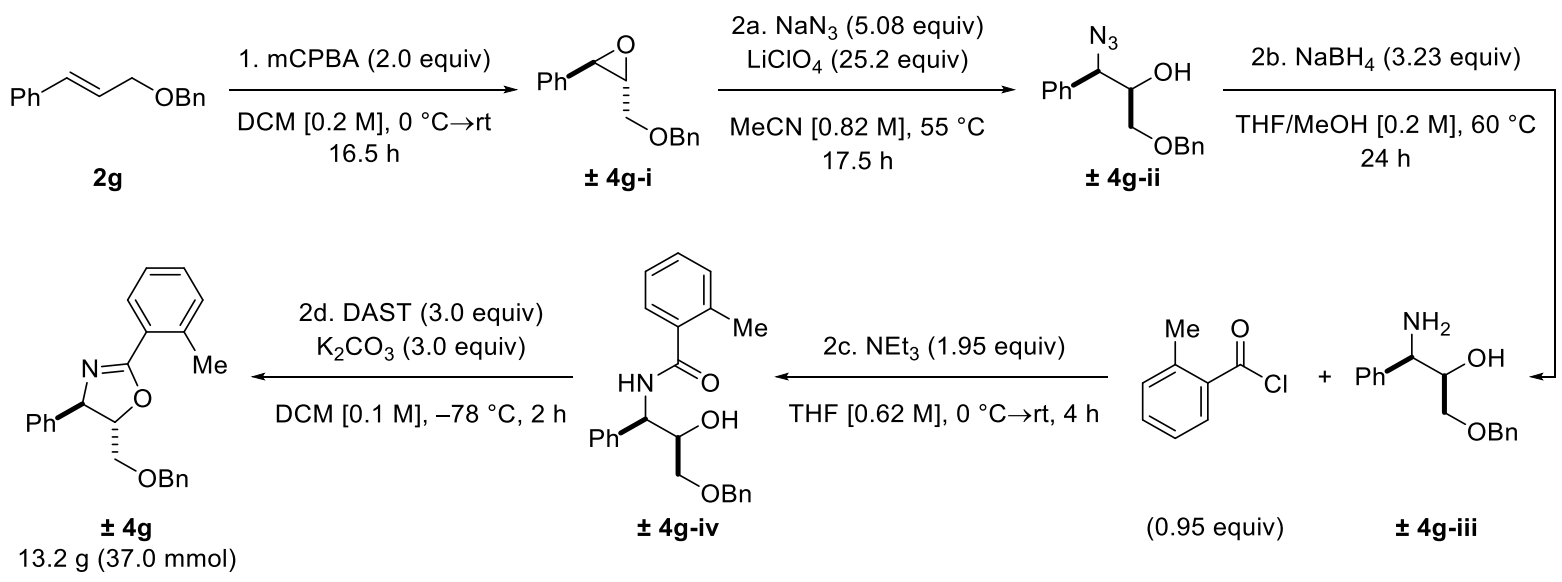

To a solution of cinnamyl benzyl ether $(\mathbf{2 g})(17.9 \mathrm{~g}, 80.0 \mathrm{mmol})$ in dichloromethane (400 $\mathrm{mL})$ in a 1 -L round-bottomed flask at $0{ }^{\circ} \mathrm{C}$ was added meta-chloroperbenzoic acid $(27.6 \mathrm{~g}, 77 \%$, $160.0 \mathrm{mmol}, 2.0$ equiv) portion-wise over $2 \mathrm{~min}$. The resulting slurry was stirred at $0{ }^{\circ} \mathrm{C}$ for 10 min, then was warmed to $21^{\circ} \mathrm{C}$ and stirred for $16.5 \mathrm{~h}$. The reaction was quenched with a sat. aq. solution of sodium bicarbonate $(150 \mathrm{~mL})$. The mixture was transferred to a 1-L separatory funnel where the layers were separated and the aqueous layer was extracted with dichloromethane ( $2 \mathrm{X}$ $200 \mathrm{~mL})$. The combined organic layers were washed with brine $(200 \mathrm{~mL})$, dried over sodium sulfate $(5 \mathrm{~g})$, filtered, and concentrated under reduced pressure by rotary evaporation $\left(30^{\circ} \mathrm{C}, \sim 20\right.$ mm Hg). Column chromatography (300 g silica gel, $5.5 \mathrm{~cm}$ column) eluting with a gradient from hexanes to hexanes/ethyl acetate, $19: 1$ (50-mL fractions) to yield $14.92 \mathrm{~g}(78 \%)$ of 2((benzyloxy)methyl)-3-phenyloxirane $( \pm \mathbf{4 g - i})$ as a clear oil. Spectral data was consistent with previous reports. ${ }^{19}$

To an anhydrous solution of $\pm \mathbf{4 g - i}(11.8 \mathrm{~g}, 49.2 \mathrm{mmol})$ in acetonitrile $(250 \mathrm{~mL})$ in a 500 mL 3-necked, round-bottomed flask was added lithium perchlorate (132.0 g, 1.24 mol, 25.2 equiv). Sodium azide (16.3 g, $250.1 \mathrm{mmol}, 5.1$ equiv) was added, followed by additional acetonitrile (60 $\mathrm{mL}$ ). The mixture was heated at $55^{\circ} \mathrm{C}$ for $17.5 \mathrm{~h}$ in an oil bath. After cooling to $21^{\circ} \mathrm{C}$, the mixture 
was decanted into a separatory funnel, rinsing the reaction flask with water $(6 \mathrm{X} 50 \mathrm{~mL})$ and diethyl ether ( 3 X $100 \mathrm{~mL})$. The layers were separated and the aqueous layer was extracted with diethyl ether $(3$ X $50 \mathrm{~mL})$. The combined organic layers were dried over magnesium sulfate $(10 \mathrm{~g})$ and concentrated under reduced pressure by rotary evaporation $\left(30^{\circ} \mathrm{C}, \sim 20 \mathrm{~mm} \mathrm{Hg}\right)$, then high vacuum $\left(21^{\circ} \mathrm{C}, \sim 0.2 \mathrm{mmHg}\right)$. The crude material of $\pm \mathbf{4 g}$-ii was used without further purification.

To an anhydrous solution of the crude $\pm \mathbf{4 g}$-ii (13.3 g, $46.8 \mathrm{mmol})$ in tetrahydrofuran (109 $\mathrm{mL}$ ) in a 500-mL 3-necked, round-bottomed flask was added sodium borohydride (5.72 g, 151.3 mmol, 3.23 equiv) under nitrogen. The mixture was heated to $60{ }^{\circ} \mathrm{C}$ in an oil bath. A methanol/tetrahydrofuran solution $(95.0: 23.8 \mathrm{~mL}-118.8 \mathrm{~mL}$ total) was added dropwise through an addition funnel over $3.5 \mathrm{~h}$. After $24 \mathrm{~h}$, the reaction was cooled to $21{ }^{\circ} \mathrm{C}$, transferred to a $1-\mathrm{L}$ separatory funnel, and was extracted with dichloromethane (3 X $50 \mathrm{~mL})$. The combined organic layers were dried over sodium sulfate ( $15 \mathrm{~g})$, filtered, and concentrated under reduced pressure by rotary evaporation $\left(30^{\circ} \mathrm{C}, \sim 20 \mathrm{~mm} \mathrm{Hg}\right)$. The crude material of $\pm \mathbf{4 g}$-iii was used without further purification.

To an anhydrous solution of the crude $\pm \mathbf{4 g - i i i ~ ( 1 1 . 9 7 ~ g , ~} 46.8 \mathrm{mmol})$ in tetrahydrofuran (71.6 $\mathrm{mL})$ in a $250-\mathrm{mL}$ 2-necked round-bottomed flask at $0{ }^{\circ} \mathrm{C}$ was added triethylamine $(12.7 \mathrm{~mL}, 91.3$ mmol, 1.95 equiv) and (2-methyl)benzoyl chloride ( $5.8 \mathrm{~mL}, 44.5 \mathrm{mmol}, 0.95$ equiv). The resulting milky white suspension was stirred at $0{ }^{\circ} \mathrm{C}$ for $5 \mathrm{~min}$, then stirred at $21^{\circ} \mathrm{C}$ for $4 \mathrm{~h}$. The mixture was concentrated, taken up in dichloromethane $(300 \mathrm{~mL})$, and transferred to a 1-L separatory funnel. Water $(150 \mathrm{~mL})$ was added and the layers were separated. The aqueous layer was extracted with dichloromethane $(2 \times 50 \mathrm{~mL})$. The combined organic layers were washed with brine (100 $\mathrm{mL})$, dried over sodium sulfate (15 g), filtered, and concentrated under reduced pressure by rotary evaporation $\left(30{ }^{\circ} \mathrm{C}, \sim 20 \mathrm{~mm} \mathrm{Hg}\right)$. The crude material of $\pm \mathbf{4 g}$-iv was used without further 
purification.

To an anhydrous solution of the crude $\pm \mathbf{4 g}$-iv ( $16.7 \mathrm{~g}, 44.5 \mathrm{mmol})$ in dichloromethane (445 $\mathrm{mL}$ ) in a $1 \mathrm{~L}$ round-bottomed flask cooled to $-78{ }^{\circ} \mathrm{C}$ in a dry ice/iso-propanol bath was added (diethylamino)sulfur trifluoride (17.6 $\mathrm{mL}, 133.5 \mathrm{mmol}, 3.0$ equiv). The mixture was stirred at -78 ${ }^{\circ} \mathrm{C}$ for $2 \mathrm{~h}$, after which potassium carbonate (18.45 g, $133.5 \mathrm{mmol}, 3.0$ equiv) was added. The slurry was stirred at $-78{ }^{\circ} \mathrm{C}$ for $10 \mathrm{~min}$, then was warmed to $21{ }^{\circ} \mathrm{C}$. A sat. aq. solution of sodium bicarbonate $(300 \mathrm{~mL})$ was added very slowly (rapid gas evolution observed beginning $\sim 30 \mathrm{sec}$ after initial addition). The mixture was transferred to a 1-L separatory funnel and the layers were separated. The aqueous layer was extracted with dichloromethane (2 X $100 \mathrm{~mL})$. The combined organic layers were washed with brine $(200 \mathrm{~mL})$, dried over sodium sulfate $(15 \mathrm{~g})$, filtered, and concentrated under reduced pressure by rotary evaporation $\left(30{ }^{\circ} \mathrm{C}, \sim 20 \mathrm{~mm} \mathrm{Hg}\right)$. Column chromatography (300 g silica gel, $5.5 \mathrm{~cm}$ column) eluting with a gradient from hexanes to ethyl acetate/hexanes, 21:4 (50-mL fractions) to yield $13.2 \mathrm{~g} \mathrm{(75 \%} \mathrm{over} 4$ steps, $37.0 \mathrm{mmol})$ of \pm trans5-((benzyloxy)methyl)-4-phenyl-2-(o-tolyl)-4,5-dihydrooxazole $(\mathbf{\pm 4 g})$ as a clear, colorless oil. Spectral data was consistent with our own internal data for the enantioenriched material (4g).

\section{General Procedure I: Synthesis for 1,2-Oxyamination of Alkenes}

A $20-\mathrm{mL}$, oven-dried reaction vial equipped with a magnetic stir bar and a septum cap was charged with 2-methyl- $N$-tosylbenzamide $(289 \mathrm{mg}, 1.00 \mathrm{mmol})$, diselenide catalyst $3 \mathbf{a}(82.1 \mathrm{mg}$, $0.10 \mathrm{mmol}, 0.10$ equiv), and alkene (if solid) (1.20 mmol, 1.20 equiv). The vial was subsequently taken into the glove box, and 1-fluoro-2,4,6-trimethylpyridinium tetrafluoroborate $(295.1 \mathrm{mg}, 1.30$ mmol, 1.30 equiv) was added. The vial was sealed and removed from the glove box. Alkene (if liquid) (1.20 mmol, 1.20 equiv) was added as a solution in $5 \mathrm{~mL}$ of 1,2-DCE or as a neat liquid 
following addition of 1,2-DCE. The reaction mixture was stirred for $24 \mathrm{~h}$ at $21^{\circ} \mathrm{C}$, then was diluted with $3 \mathrm{~mL}$ of dichloromethane and was concentrated under reduced pressure by rotary evaporation $\left(25-30^{\circ} \mathrm{C}, \sim 20 \mathrm{~mm} \mathrm{Hg}\right)$. The crude product was purified by silica gel column chromatography and subsequent diffusion pump distillation or recrystallization to afford analytically pure products.

\section{Survey of $\mathrm{N}$-Sulfonamide Benzamides (Table 2)}

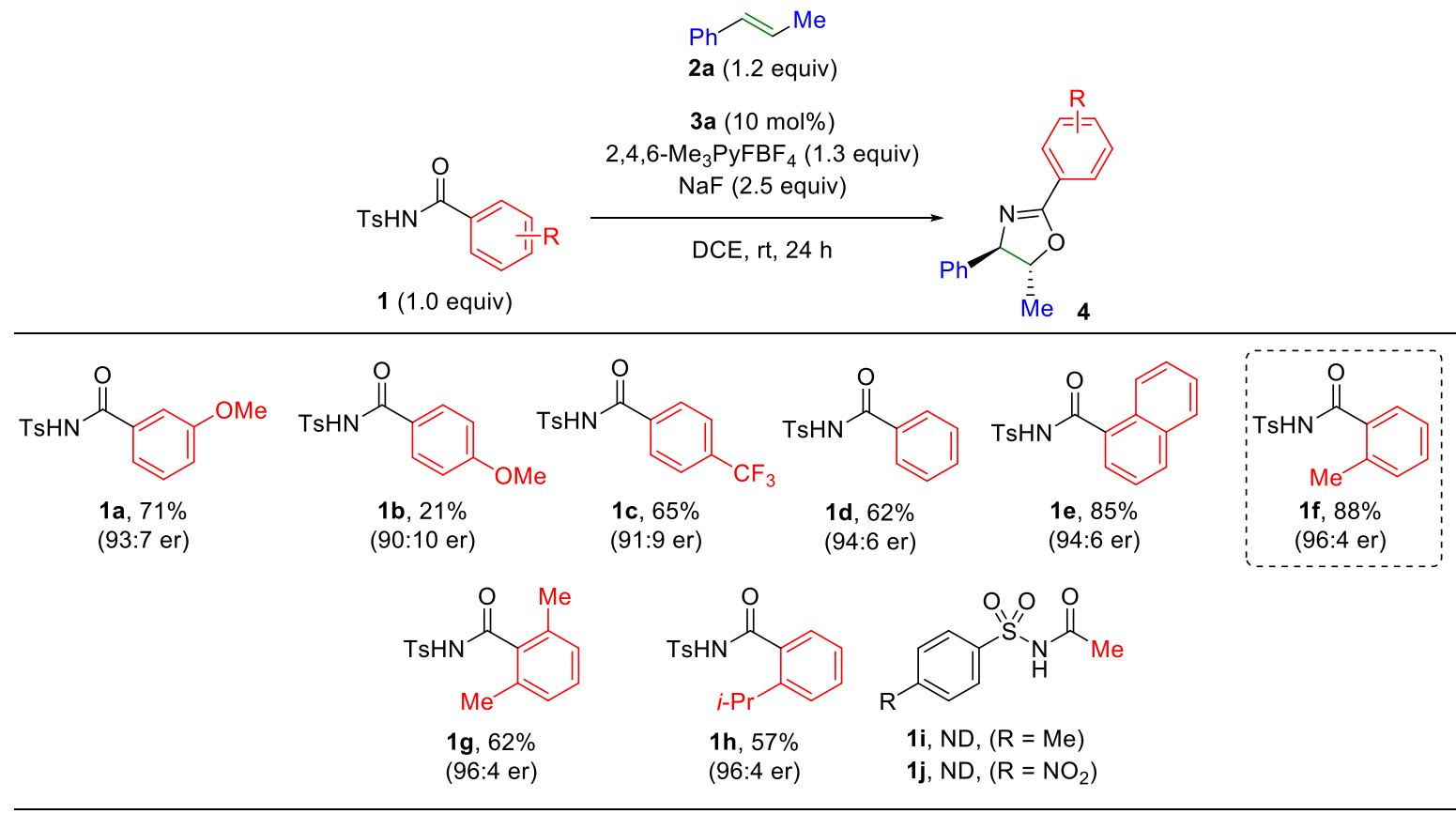

Following a modification of General Procedure I, $N$-tosylbenzamide $(0.20 \mathrm{mmol})$, diselenide catalyst 3a (16.4 mg, $0.02 \mathrm{mmol}, 0.10$ equiv), 2,4,6- $\mathrm{Me}_{3} \mathrm{PyF}^{+} \mathrm{BF}_{4}^{-}$(59.0 mg, $0.26 \mathrm{mmol}$, 1.30 equiv), sodium fluoride (21.0 mg, $0.5 \mathrm{mmol}, 2.5$ equiv), and $\beta$-methylstyrene (28.4 mg, 0.24 mmol, 1.20 equiv) were combined in 1,2-DCE $(1.0 \mathrm{~mL}, 0.20 \mathrm{M})$ (alkene added neat) and allowed to stir for $24 \mathrm{~h}$ at $21^{\circ} \mathrm{C}$. The resulting suspension was worked up as described in the General Procedure.

For 1a: Purified by column chromatography (30 g silica gel, $2 \mathrm{~cm}$ column) eluting with hexanes/ethyl acetate, 8:1 (20-mL fractions) to yield $37.7 \mathrm{mg}(71 \%)$ as a yellow oil. CSP-HPLC 
run with Regis $R, R$-Whelk-O1, hexanes/i-PrOH, 70:30, $220 \mathrm{~nm}, 24{ }^{\circ} \mathrm{C}$.

For 1b: Purified by column chromatography (30 g silica gel, $2 \mathrm{~cm}$ column) eluting with hexanes/diethyl ether, 4:1 (10-mL fractions). Prep TLC run with 1000 micron plate and dichloromethane. Prep TLC run with 1000 micron plate and hexanes/ethyl acetate to yield 11.2 $\operatorname{mg}(21 \%)$.

For 1c: Purified by column chromatography (30 g silica gel, $2 \mathrm{~cm}$ column) eluting with hexanes/ethyl acetate, 8:1 (10-mL fractions). Further purified by column chromatography (30 g silica gel, $2 \mathrm{~cm}$ column) eluting with chloroform/ethyl acetate, 99:1 (10-mL fractions). Prep TLC run with 1000 micron plate and hexanes/ethyl acetate, $97: 3$ to yield $39.5 \mathrm{mg}(65 \%)$ as a white solid. CSP-HPLC run with Regis $R, R$-Whelk-O1, hexanes $/ i-\mathrm{PrOH}, 70: 30,220 \mathrm{~nm}, 24{ }^{\circ} \mathrm{C}$.

For 1d: Purified by column chromatography (30 g silica gel, $2 \mathrm{~cm}$ column) eluting with hexanes/ethyl acetate, 8:1 (10-mL fractions). Prep TLC run with 1000 micron plate and dichloromethane to yield $29.5 \mathrm{mg}(62 \%)$ as a clear oil. CSP-HPLC run with Regis $R, R$-Whelk-O1, hexanes/i-PrOH, 70:30, $220 \mathrm{~nm}, 24{ }^{\circ} \mathrm{C}$.

For 1e: Purified by column chromatography on Teledyne ISCO Combi-Flash (silica gel, 25 g Luknova SuperSep column) eluting with a gradient of hexanes/ethyl acetate, 95:5 to 90:10 (20-mL fractions). Prep TLC run with hexanes/ethyl acetate, 8:1. Prep TLC run with chloroform/ethyl acetate, $97: 3$ to yield $48.8 \mathrm{mg}(85 \%)$ as a yellow oil. CSP-HPLC run with Supelco Astec Cellulose, hexanes/i-PrOH, 70:30, $220 \mathrm{~nm}, 24^{\circ} \mathrm{C}$.

For 1f: Purified by column chromatography on Teledyne ISCO Combi-Flash (silica gel, 25 g Luknova SuperSep column) eluting with a gradient of hexanes/ethyl acetate, 95:5 to 90:10 (20$\mathrm{mL}$ fractions). Prep TLC run with dichloromethane to yield $44.0 \mathrm{mg}(88 \%)$. CSP-HPLC run with Regis $R, R$-Whelk-O1, hexanes/i-PrOH, 70:30, $220 \mathrm{~nm}, 24^{\circ} \mathrm{C}$. 
For 1g: Purified by column chromatography (45 g silica gel, $2 \mathrm{~cm}$ column) eluting with chloroform/ethyl acetate, 97:3 (10-mL fractions). Prep TLC run with dichloromethane to yield $32.7 \mathrm{mg}(62 \%)$.

For 1h: Purified by column chromatography on Teledyne ISCO Combi-Flash (silica gel, 25 g Luknova SuperSep column) eluting with a gradient from hexanes to hexanes/ethyl acetate, 95:5 (20-mL fractions) to yield $31.8 \mathrm{mg}(57 \%)$ of a clear oil.

\section{Survey of Chiral Diselenide Catalysts (Table 3)}

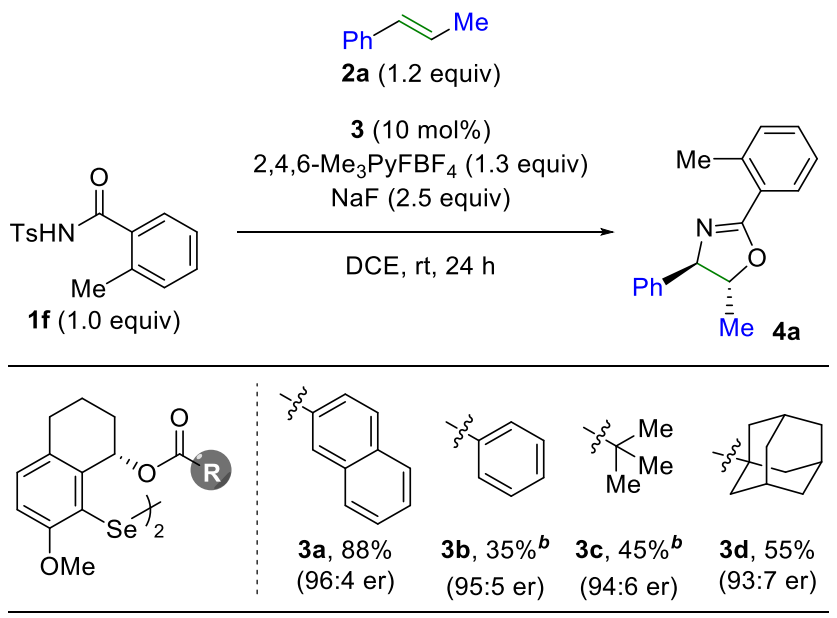

Following a modification of General Procedure I, 2-methyl- $N$-tosylbenzamide $(57.9 \mathrm{mg}$, $0.20 \mathrm{mmol}$ ), diselenide catalyst 3 (0.02 mmol, 0.10 equiv), $2,4,6-\mathrm{Me}_{3} \mathrm{PyF}^{+} \mathrm{BF}_{4}^{-}(59.0 \mathrm{mg}, 0.26$ mmol, 1.30 equiv), sodium fluoride (21.0 mg, $0.5 \mathrm{mmol}, 2.5$ equiv), and $\beta$-methylstyrene (28.4 mg, $0.24 \mathrm{mmol}, 1.20$ equiv) were combined in 1,2-DCE (1.0 mL, $0.20 \mathrm{M})$ (alkene added neat) and allowed to stir for $24 \mathrm{~h}$ at $21{ }^{\circ} \mathrm{C}$. The resulting suspension was worked up as described in the General Procedure and purified by column chromatography on Teledyne ISCO Combi-Flash (silica gel, 4 g Luknova SuperSep column) eluting with a gradient from hexanes to hexanes/ethyl acetate, 95:5 (8-mL fractions) to yield $\mathbf{4 a}$ as a clear, colorless oil. 


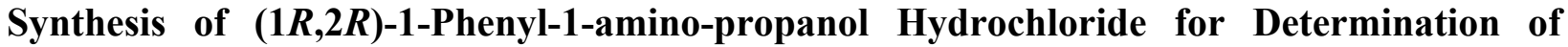

\section{Absolute Configuration of the Oxazoline Products.}

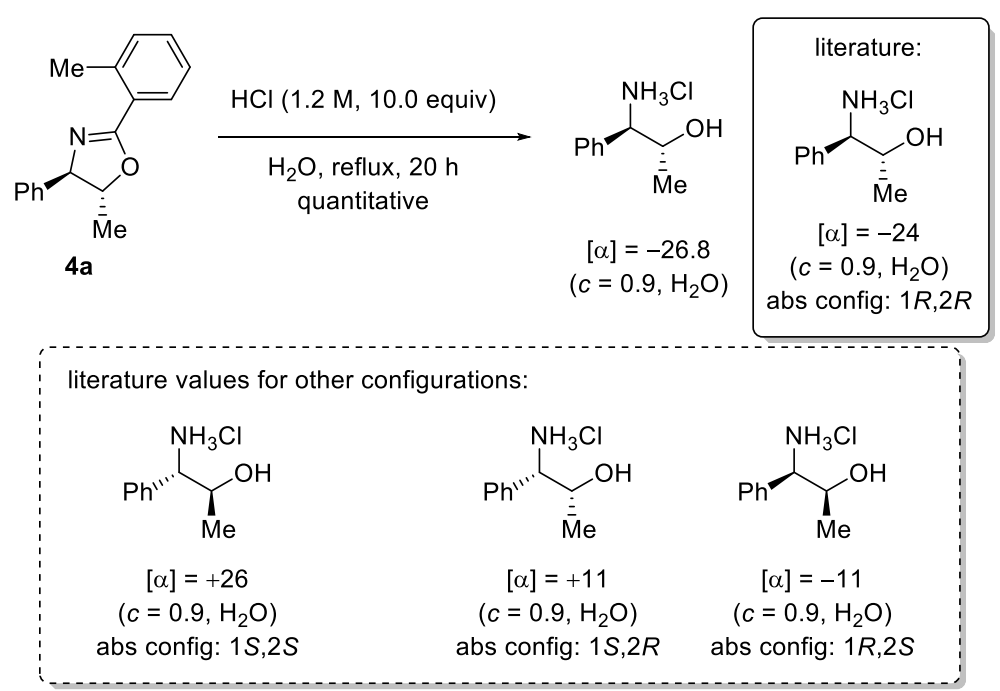

Following a protocol for a related hydrolysis, ${ }^{20}$ (4R,5R)-5-methyl-4-phenyl-2-(2methylphenyl)-4,5-dihydrooxazole (4a) (50.3 mg, $0.2 \mathrm{mmol}, 96: 4$ er) was treated with aqueous hydrochloric acid (1.2 M, $2.0 \mathrm{mmol}, 10.0$ equiv). The resulting mixture was heated at $115^{\circ} \mathrm{C}$ for $20 \mathrm{~h}$. After cooling to room temperature, a white precipitate formed and was filtered off, washing the precipitate with water $(3 \mathrm{X} 1 \mathrm{~mL})$. The filtrate was washed sequentially with ethyl acetate $(5$ $\mathrm{mL})$, diethyl ether $(5 \mathrm{~mL})$, and dichloromethane $(5 \mathrm{~mL})$. The aqueous layer was concentrated under reduced pressure by rotary evaporation $\left(40{ }^{\circ} \mathrm{C}, \sim 20 \mathrm{~mm} \mathrm{Hg}\right)$, then high vacuum $\left(75^{\circ} \mathrm{C}, \sim 0.2\right.$ $\mathrm{mmHg}$ ) to yield $(1 R, 2 R)$-1-phenyl-1-amino-propanol hydrochloride (39.9 mg).

The optical rotation of a solution of $(1 R, 2 R)$-1-phenyl-1-amino-propanol hydrochloride $(18.0 \mathrm{mg})$ in water $(2 \mathrm{~mL})$ was measured to be $[\alpha]_{\mathrm{D}}^{23}=-26.8\left(\mathrm{c}=0.9, \mathrm{H}_{2} \mathrm{O}\right)$ which is similar to the reported value of $[\alpha]_{\mathrm{D}}{ }^{23}=-24\left(\mathrm{c}=0.9, \mathrm{H}_{2} \mathrm{O}\right)$ for $1 R, 2 R .^{21}$ 


\section{Effect for NaF on the Alkene 1,2-Oxyamination Reaction}
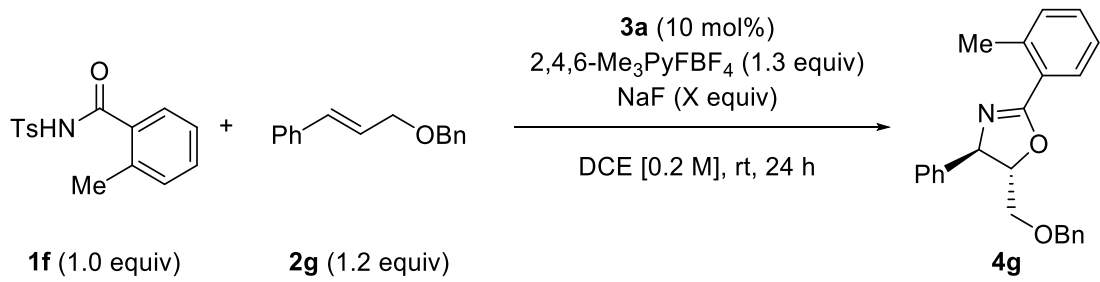

Following a modification of General Procedure I, 2-methyl- $N$-tosylbenzamide $(57.9 \mathrm{mg}$, $0.20 \mathrm{mmol}$ ), diselenide catalyst 3a (16.4 mg, $0.02 \mathrm{mmol}, 0.10$ equiv), $2,4,6-\mathrm{Me}_{3} \mathrm{PyF}^{+} \mathrm{BF}_{4}^{-}(59.0$ mg, 0.26 mmol, 1.30 equiv), sodium fluoride (corresponding amount below), and cinnamyl benzyl ether $(53.8 \mathrm{mg}, 0.24 \mathrm{mmol}, 1.20$ equiv) were combined in 1,2-DCE (1.0 mL, $0.20 \mathrm{M})$ (alkene added as a solution in DCE) and allowed to stir for $24 \mathrm{~h}$ at $21{ }^{\circ} \mathrm{C}$. The resulting suspension was worked up as described in the General Procedure. 1,1,2,2-Tetrachloroethane $(21.1 \mu \mathrm{L}, 0.20 \mathrm{mmol}$, 1.00 equiv) was added and the sample was analyzed for yield by ${ }^{1} \mathrm{H}$ NMR by comparison of peak integrations (8 scans, 5 second relaxation delay).

\begin{tabular}{ccc}
\hline entry & NaF (equiv) & Yield (\%) \\
\hline 1 & 0.0 & 60 \\
2 & 0.5 & 64 \\
3 & 1.0 & 62 \\
4 & 1.5 & 65 \\
5 & 2.5 & 64 \\
6 & 5.0 & 63 \\
\hline
\end{tabular}

\section{Kinetics plot}

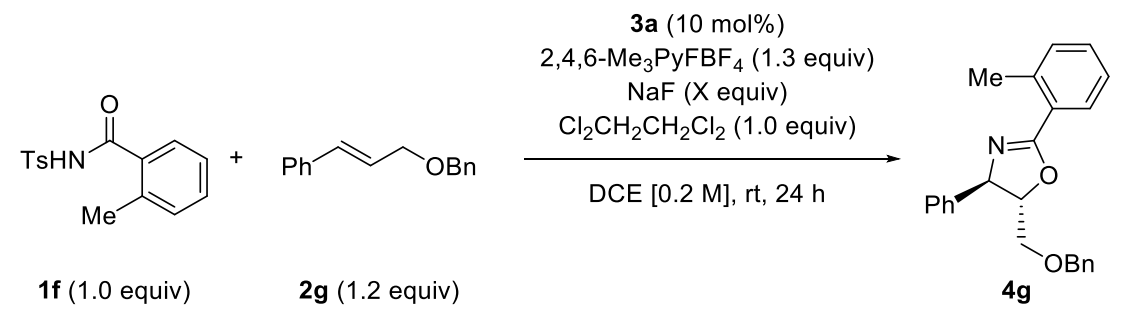

Following a modification of General Procedure I, 2-methyl- $N$-tosylbenzamide $(57.9 \mathrm{mg}$, $0.20 \mathrm{mmol}$ ), diselenide catalyst $3 \mathrm{a}$ (16.4 mg, $0.02 \mathrm{mmol}, 0.10$ equiv), $2,4,6-\mathrm{Me}_{3} \mathrm{PyF}^{+} \mathrm{BF}_{4}^{-}(59.0$ 
mg, 0.26 mmol, 1.30 equiv), sodium fluoride (corresponding amount below), 1,1,2,2tetrachloroethane $(21.1 \mu \mathrm{L}, 0.20 \mathrm{mmol}, 1.00$ equiv), and cinnamyl benzyl ether $(53.8 \mathrm{mg}, 0.24$ mmol, 1.20 equiv) were combined in 1,2-DCE $(1.0 \mathrm{~mL}, 0.20 \mathrm{M})$ (alkene added as a solution in DCE) and allowed to stir for $24 \mathrm{~h}$ at $21^{\circ} \mathrm{C}$. At the marked timepoints, a $50 \mu \mathrm{L}$ aliquot was removed from the reaction, added to $0.4 \mathrm{~mL} \mathrm{CDCl}_{3}$ and analyzed for yield by ${ }^{1} \mathrm{H} \mathrm{NMR}$ by comparison of peak integrations (solvent suppression of DCE peak, 10 satpwr, 8 scans, 5 s relaxation delay).

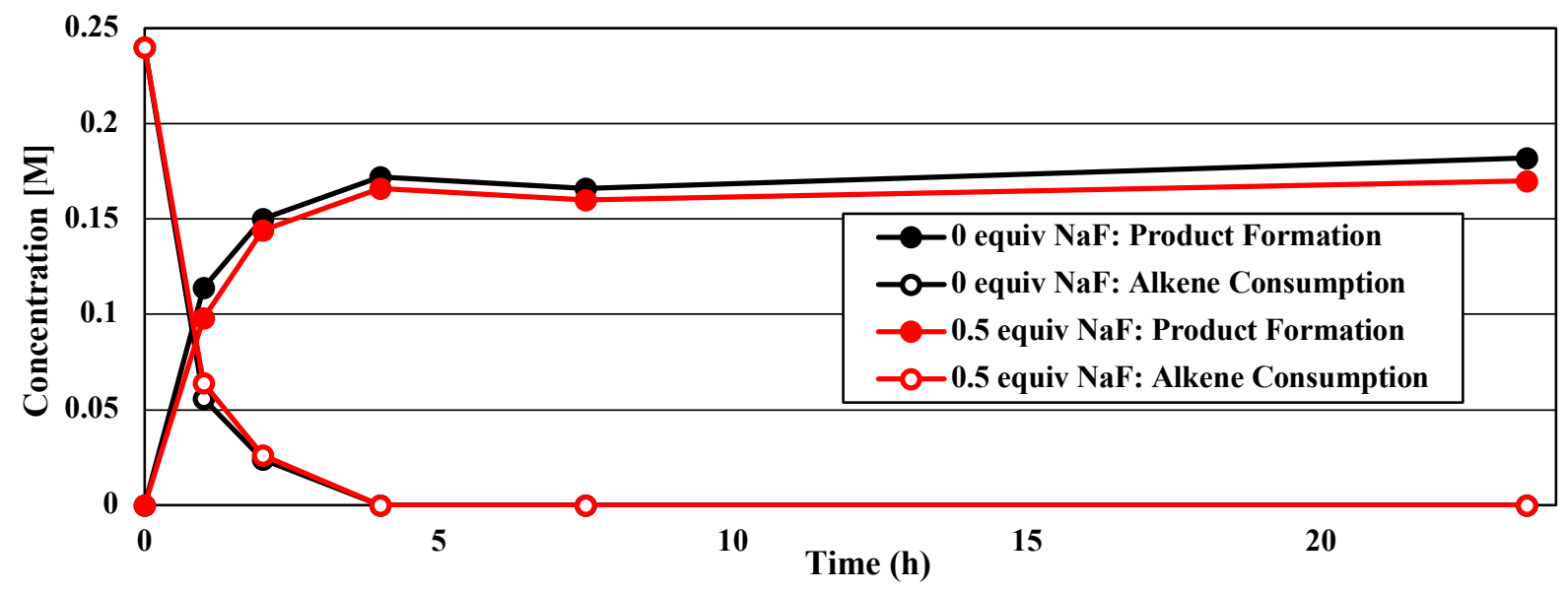

\section{General Procedure for Catalytic Oxyamination}

\section{Preparation of (4R,5R)-5-Methyl-4-phenyl-2-(2-methylphenyl)-4,5-dihydrooxazole (4a).}

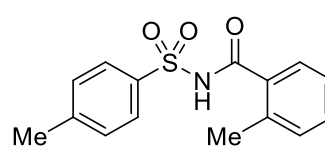

$1 f$ ( 1.0 equiv)

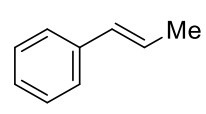

2a (1.2 equiv)

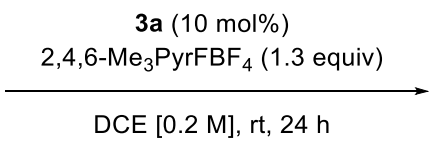

DCE [0.2 M], rt, $24 \mathrm{~h}$
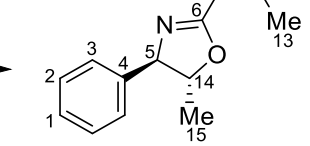

$4 \mathrm{a}$

Following General Procedure I, 2-methyl- $N$-tosylbenzamide (289 mg, $1.00 \mathrm{mmol})$, diselenide catalyst 3a $\left(82.1 \mathrm{mg}, 0.10 \mathrm{mmol}, 0.10\right.$ equiv), 2,4,6- $\mathrm{Me}_{3} \mathrm{PyF}^{+} \mathrm{BF}_{4}^{-}$(295 mg, $1.30 \mathrm{mmol}$, 1.30 equiv), and trans- $\beta$-methylstyrene (156 $\mu \mathrm{L}, 1.20 \mathrm{mmol}, 1.20$ equiv) were combined in 1,2DCE $\left(5.0 \mathrm{~mL}, 0.20 \mathrm{M}\right.$ ) (alkene was added as a neat liquid) and allowed to stir for $24 \mathrm{~h}$ at $21{ }^{\circ} \mathrm{C}$. 
The resulting suspension was worked up as described in the General Procedure and purified by column chromatography on Teledyne ISCO Combi-Flash (silica gel, 120 g Luknova SuperSep column) eluting with a gradient from hexanes to hexanes/ethyl acetate, 95:5 (20-mL fractions) to yield $171 \mathrm{mg}(68 \%)$ of $\mathbf{4 a}$ as a clear, colorless oil. A $50.0 \mathrm{mg}$ portion was further purified by Kugelrohr distillation $\left(68{ }^{\circ} \mathrm{C}, 2.9 \times 10^{-5} \mathrm{~mm} \mathrm{Hg}\right)$ to afford $49.1 \mathrm{mg}$ of a clear colorless oil $(98 \%$ recovery).

Data for 4a:

b.p. $68^{\circ} \mathrm{C}\left(2.9 \times 10^{-5} \mathrm{~mm} \mathrm{Hg}\right)$

1H NMR: $\quad\left(500 \mathrm{MHz}, \mathrm{CDCl}_{3}\right)$

$\delta 7.89(\mathrm{dd}, J=7.8,1.4 \mathrm{~Hz}, 1 \mathrm{H}, \mathrm{HC}(8))$, 7.39-7.34 (m, $3 \mathrm{H}, \mathrm{HC}(2,10))$, 7.347.29 (m, $3 \mathrm{H}, \mathrm{HC}(1,3)) ; 7.29-7.23$ (m, $2 \mathrm{H}, \mathrm{HC}(9,11)), 4.89$ (d, J = 7.3 Hz, $1 \mathrm{H}$, $\mathrm{HC}(5)), 4.54$ (dq, $J=7.3,6.2 \mathrm{~Hz}, 1 \mathrm{H}, \mathrm{HC}(14)), 2.66$ (s, $\left.3 \mathrm{H}, \mathrm{H}_{3} \mathrm{C}(13)\right), 1.57$ (d, $\left.J=6.1 \mathrm{~Hz}, 3 \mathrm{H}, \mathrm{H}_{3} \mathrm{C}(15)\right)$.

${ }^{13} \mathrm{C} \mathrm{NMR}: \quad\left(126 \mathrm{MHz}, \mathrm{CDCl}_{3}\right)$

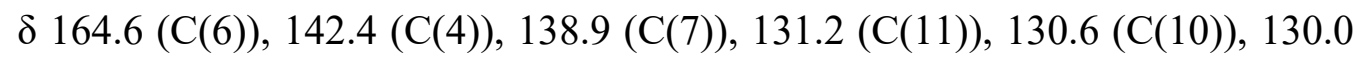
(C(8)), $128.7(\mathrm{C}(2)), 127.5(\mathrm{C}(1)), 127.3(\mathrm{C}(12)), 126.5(\mathrm{C}(3)), 125.6(\mathrm{C}(9)), 83.3$ (C(14)), $77.5(\mathrm{C}(5)), 21.9(\mathrm{C}(13)), 20.9(\mathrm{C}(15))$.

IR: (neat)

$3062(w), 3028(w), 2971(w), 2925(w), 1637(s), 1602(w), 1574(w), 1494(m)$, 1453 (m), 1381 (w), 1342 (w), 1317 (w), 1303 (m), 1277 (m), 1233 (m), 1201 (w), $1162(w), 1138(w), 1122(w), 1043(s), 1006(m), 942(w), 912(w), 890$ (w), $872(\mathrm{~m}), 837(\mathrm{w}), 776(\mathrm{~m}), 752(\mathrm{~m}), 728(\mathrm{~s}), 698(\mathrm{~s}), 632(\mathrm{w}), 561(\mathrm{w}), 538$ (w), $504(\mathrm{w}), 471(\mathrm{w})$ 
HRMS: $\quad\left(\mathrm{ESI}^{+}\right)$Cald for $\mathrm{C}_{17} \mathrm{H}_{18} \mathrm{NO}^{+}: 252.1388$, found: 252.1376.

Analysis: $\mathrm{C}_{17} \mathrm{H}_{17} \mathrm{NO}$

Calcd: C, 81.24\%; H, 6.82\%; N, 5.57\%

Found: $\mathrm{C}, 81.00 \% ; \mathrm{H}, 6.80 \% ; \mathrm{N}, 5.82 \%$

TLC: $\quad R_{f}=0.47(16: 1$, hexanes/ethyl acetate $)[\mathrm{UV}]$

CSP-HPLC: $\quad(R, R)-\mathbf{4 a} t_{R}=4.96 \min (96.1 \%),(S, S)-\mathbf{4 a} t_{R}=6.23 \mathrm{~min}(3.9 \%)($ Regis $R, R$-WhelkO1, hexanes/i-PrOH, 70:30, $\left.1.0 \mathrm{~mL} / \mathrm{min} .220 \mathrm{~nm}, 24^{\circ} \mathrm{C}\right)$.

Opt. Rot.: $[\alpha]_{\mathrm{D}}^{23} 22.7\left(c=0.64, \mathrm{CHCl}_{3}\right)$

Preparation of (4R,5R)-5-(2-Methylethyl)-2-(2-methylphenyl)-phenyl-4,5-dihydrooxazole (4b).

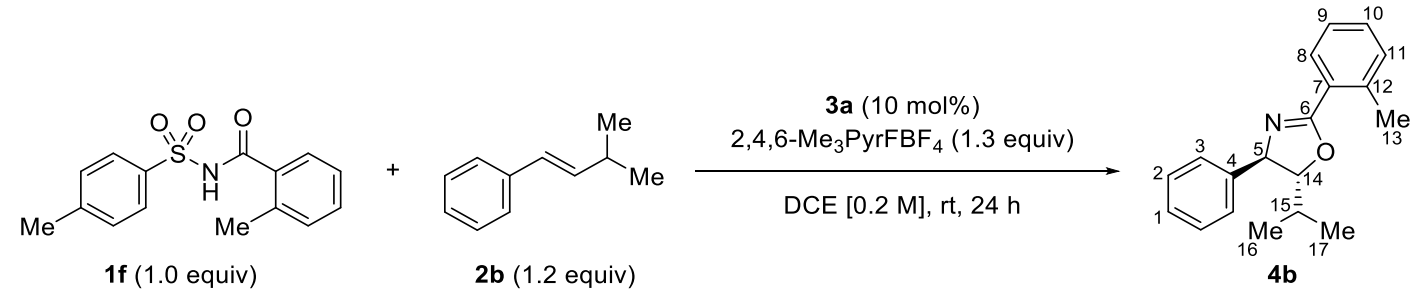

Following General Procedure I, 2-methyl- $N$-tosylbenzamide (289 mg, $1.00 \mathrm{mmol}$ ), diselenide catalyst $3 \mathbf{a}\left(82.1 \mathrm{mg}, 0.10 \mathrm{mmol}, 0.10\right.$ equiv), 2,4,6- $\mathrm{Me}_{3} \mathrm{PyF}^{+} \mathrm{BF}_{4}^{-}(295 \mathrm{mg}, 1.30 \mathrm{mmol}$, 1.30 equiv), and (E)-(3-methylbut-1-en-1-yl)benzene (175 mg, $1.20 \mathrm{mmol}, 1.20$ equiv) were combined in 1,2-DCE $(5.0 \mathrm{~mL}, 0.20 \mathrm{M})$ (alkene was added as a neat liquid) and allowed to stir for $24 \mathrm{~h}$ at $21^{\circ} \mathrm{C}$. The resulting suspension was worked up as described in the General Procedure and purified by column chromatography (160 g silica gel, $5.5 \mathrm{~cm}$ column) eluting with a gradient of hexanes/ethyl acetate, $97: 3$ to $94: 6(20-\mathrm{mL}$ fractions) to yield $234 \mathrm{mg}(84 \%)$ of $\mathbf{4 b}$ as a clear, colorless oil. A $100.0 \mathrm{mg}$ portion was further purified by Kugelrohr distillation $\left(76{ }^{\circ} \mathrm{C}, 3.4 \times 10^{-5}\right.$ $\mathrm{mm} \mathrm{Hg}$ ) to afford $99.5 \mathrm{mg}$ of a clear colorless oil (99\% recovery). 


\section{Data for $4 \mathbf{b}$ :}

b.p. $76^{\circ} \mathrm{C}\left(3.4 \times 10^{-5} \mathrm{~mm} \mathrm{Hg}\right)$

${ }^{1} \mathrm{H}$ NMR: $\quad\left(500 \mathrm{MHz}, \mathrm{CDCl}_{3}\right)$

$\delta 7.97(\mathrm{~d}, J=7.7 \mathrm{~Hz}, 1 \mathrm{H}, \mathrm{HC}(8)), 7.40-7.33(\mathrm{~m}, 3 \mathrm{H}, \mathrm{HC}(2,10)), 7.32-7.24(\mathrm{~m}$,

$5 \mathrm{H}, \mathrm{HC}(1,3,8,9,11)), 5.05(\mathrm{~d}, J=6.4 \mathrm{~Hz}, 1 \mathrm{H}, \mathrm{HC}(5)), 4.31(\mathrm{t}, J=6.3 \mathrm{~Hz}, 1$

H, HC(14)), 2.66 (s, 3 H, H $\left.{ }_{3} \mathrm{C}(13)\right), 2.03$ (hex, $J=6.7$ Hz, $\left.1 \mathrm{H}, \mathrm{HC}(15)\right), 1.09$ (d, $J=6.7 \mathrm{~Hz}, 3 \mathrm{H}, \mathrm{H}_{3} \mathrm{C}(16$ or 17$\left.)\right), 1.06\left(\mathrm{~d}, J=6.7 \mathrm{~Hz}, 3 \mathrm{H}, \mathrm{H}_{3} \mathrm{C}(16\right.$ or 17$\left.)\right)$.

${ }^{13} \mathrm{C} \mathrm{NMR:} \quad\left(126 \mathrm{MHz}, \mathrm{CDCl}_{3}\right)$

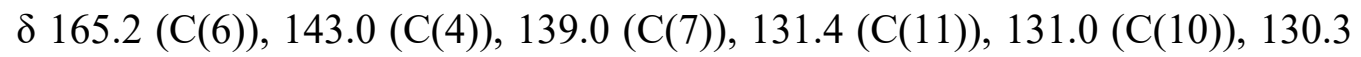

(C(8)), $128.8(\mathrm{C}(2)), 127.5(\mathrm{C}(1)), 126.8(\mathrm{C}(3,12)), 125.7(\mathrm{C}(9)), 92.1(\mathrm{C}(14))$ $72.8(\mathrm{C}(5)), 32.7(\mathrm{C}(15)), 22.0(\mathrm{C}(13)), 17.9(\mathrm{C}(16$ or 17$)), 17.4(\mathrm{C}(16$ or 17$))$.

IR: (neat)

$3062(w), 3029(w), 2962(w), 2927(w), 2874(w), 1639(s), 1602(w), 1575(w)$, 1493 (m), 1453 (m), 1389 (w), 1369 (w), 1346 (w), 1329 (w), 1308 (w), 1272 (m), $1227(\mathrm{w}), 1200(\mathrm{w}), 1177(\mathrm{w}), 1162(\mathrm{w}), 1138(\mathrm{w}), 1122(\mathrm{w}), 1075(\mathrm{w})$, $1050(\mathrm{~s}), 980(\mathrm{~m}), 930(\mathrm{w}), 919(\mathrm{w}), 895(\mathrm{w}), 860(\mathrm{w}), 841(\mathrm{w}), 755(\mathrm{~m}), 727(\mathrm{~s})$, $697(\mathrm{~s}), 681(\mathrm{~m}), 626(\mathrm{w}), 607(\mathrm{w}), 566(\mathrm{w}), 525(\mathrm{w}), 456(\mathrm{w})$

HRMS: $\quad\left(\mathrm{ESI}^{+}\right)$Cald for $\mathrm{C}_{19} \mathrm{H}_{22} \mathrm{NO}^{+}: 280.1701$, found: 280.1704 .

Analysis: $\mathrm{C}_{19} \mathrm{H}_{21} \mathrm{NO}$

Calcd: C, 81.68\%; H, 7.58\%; N, 5.01\%

Found: C, $81.66 \% ; \mathrm{H}, 7.62 \% ; \mathrm{N}, 5.31 \%$

$\underline{\text { TLC: }} \quad R_{f}=0.29(16: 1$, hexanes/ethyl acetate $)[\mathrm{UV}]$

CSP-HPLC: $\quad(R, R)-4 \mathbf{b} t_{R}=4.45 \min (96.6 \%),(S, S)-4 \mathbf{b} t_{R}=6.15 \min (3.43 \%)($ Regis $R, R-$ 
Whelk-O1, hexanes $\left./ i-\mathrm{PrOH}, 70: 30,1.0 \mathrm{~mL} / \mathrm{min} .220 \mathrm{~nm}, 24^{\circ} \mathrm{C}\right)$.

Opt. Rot.: $[\alpha]_{\mathrm{D}}^{23} 61.8\left(c=1.15, \mathrm{CHCl}_{3}\right)$

Preparation of 4-Methyl- $N$-phenyl- $N-(((4 R, 5 R)-2-(2-m e t h y l p h e n y l)-4-p h e n y l-4,5-$ dihydrooxazol-5-yl)methyl)benzenesulfonamide (4c).

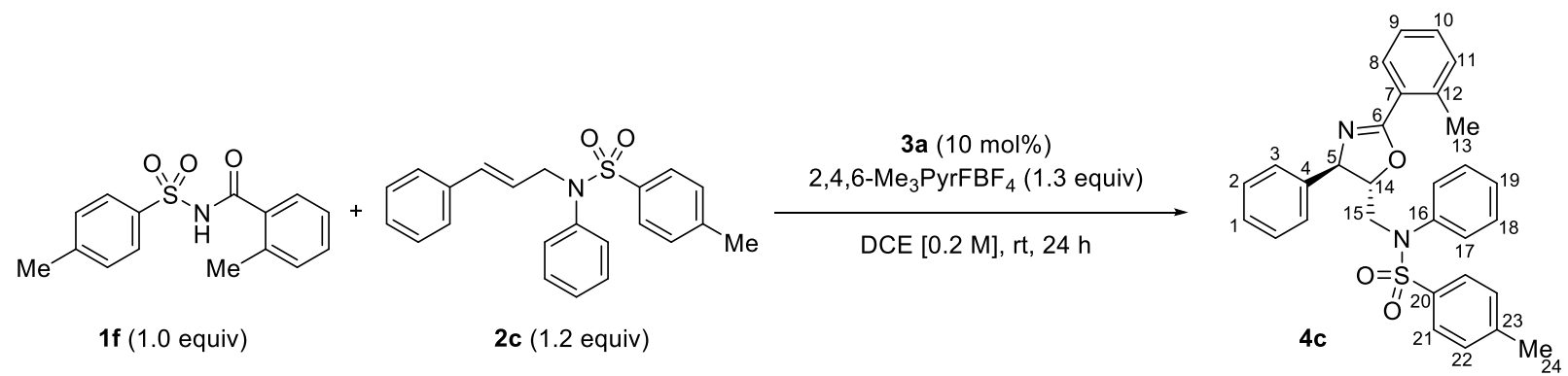

Following General Procedure I, 2-methyl- $N$-tosylbenzamide (289 mg, $1.00 \mathrm{mmol}$ ), diselenide catalyst 3a (82.1 mg, 0.10 mmol, 0.10 equiv), 2,4,6- $\mathrm{Me}_{3} \mathrm{PyF}^{+} \mathrm{BF}_{4}^{-}(295 \mathrm{mg}, 1.30 \mathrm{mmol}$, 1.30 equiv), and $N$-cinnamyl-4-methyl- $N$-phenylbenzenesulfonamide (436 mg, $1.20 \mathrm{mmol}, 1.20$ equiv) were combined in 1,2-DCE $(5.0 \mathrm{~mL}, 0.20 \mathrm{M})$ and allowed to stir for $24 \mathrm{~h}$ at $21{ }^{\circ} \mathrm{C}$. The resulting suspension was worked up as described in the General Procedure and purified by column chromatography (150 g silica gel, $5.5 \mathrm{~cm}$ column) eluting with a gradient from hexanes to hexanes/ethyl acetate, 80:20 (50-mL fractions) to yield $454 \mathrm{mg}(91 \%)$ of $\mathbf{4 c}$ as a white solid. The solid was purified to analytical purity through precipitation by dissolving in a minimal amount of ethyl acetate and adding hexanes to precipitation. The white powder precipitate was filtered off and dried.

Data for 4c:

m.p. $147^{\circ} \mathrm{C}$ (hexane) 
래 NMR: $\quad\left(500 \mathrm{MHz}, \mathrm{CDCl}_{3}\right)$

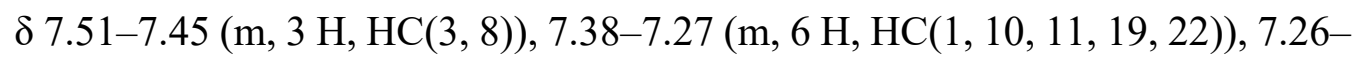
7.20 (m, $6 \mathrm{H}, \mathrm{HC}(17,18,21)), 7.13(\mathrm{t}, J=7.6 \mathrm{~Hz}, 1 \mathrm{H}, \mathrm{HC}(9))$, 7.06-7.02 (m, 2 H, $\mathrm{HC}(2)), 5.33$ (d, $J=6.5 \mathrm{~Hz}, 1 \mathrm{H}, \mathrm{HC}(5)), 4.52$ (q, $J=5.8 \mathrm{~Hz}, 1 \mathrm{H}, \mathrm{HC}(14)) 3.96$ (d, $\left.J=5.7 \mathrm{~Hz}, 2 \mathrm{H}, \mathrm{H}_{2} \mathrm{C}(15)\right), 2.56$ (s, $\left.3 \mathrm{H}, \mathrm{H}_{3} \mathrm{C}(13)\right), 2.42$ (s, $\left.3 \mathrm{H}, \mathrm{H}_{3} \mathrm{C}(24)\right)$.

${ }^{13} \mathrm{C}$ NMR: $\quad\left(126 \mathrm{MHz}, \mathrm{CDCl}_{3}\right)$

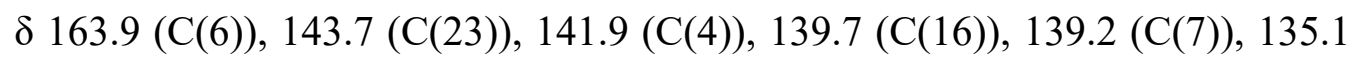
(C(20)), $131.2(\mathrm{C}(11)), 130.7(\mathrm{C}(10)), 130.1(\mathrm{C}(8)), 129.5(\mathrm{C}(22)), 129.1(\mathrm{C}(17$ or 18 or 21$)), 128.9(\mathrm{C}(17$ or 18 or 21$)), 128.7(\mathrm{C}(2)), 128.1(\mathrm{C}(19)), 127.8(\mathrm{C}(17$ or 18 or 21$)), 127.6(\mathrm{C}(12)), 126.7(\mathrm{C}(3)), 126.4(\mathrm{C}(1)), 125.4(\mathrm{C}(9)), 84.1$ (C(14)), $73.5(\mathrm{C}(5)), 53.7(\mathrm{C}(15)), 22.1(\mathrm{C}(13)), 21.6(\mathrm{C}(24))$.

IR: (thin-film)

3030 (w), 2925 (w), 1642 (m), 1597 (w), 1492 (m), 1453 (m), 1275 (m), 1228 (w), $1163(\mathrm{~s}), 1091(\mathrm{~m}), 1073(\mathrm{~m}), 1043$ (s), 908 (m), 872 (w), 815 (m), 767 (m), $727(\mathrm{~s}), 697$ (s), $656(\mathrm{~s}), 597$ (w), $572(\mathrm{~s}), 547$ (s).

HRMS: $\quad\left(\mathrm{ESI}^{+}\right.$) Calcd for $\mathrm{C}_{30} \mathrm{H}_{29} \mathrm{~N}_{2} \mathrm{O}_{3} \mathrm{~S}^{+}: 497.1899$, found: 497.1896.

Analysis: $\mathrm{C}_{30} \mathrm{H}_{28} \mathrm{~N}_{2} \mathrm{O}_{3} \mathrm{~S}$

Calcd: C, 72.55\%; H, 5.68\%; N, 5.64\%

Found: C, $72.30 \%, \mathrm{H}, 5.71 \%$;, $5.72 \%$

TLC: $\quad R_{f}=0.41\left(80: 20\right.$ hexanes/ethyl acetate) $\left[\mathrm{UV} / \mathrm{KMnO}_{4}\right]$

CSP-HPLC: $\quad(S, S)-\mathbf{4 c} t_{R}=16.4 \min (2.8 \%),(R, R)-4 \mathbf{c} t_{R}=19.2 \min (97.2 \%)$ (Supelco Astec, hexanes $\left./ i-\mathrm{PrOH}, 90: 10,1.0 \mathrm{~mL} / \mathrm{min} ; 220 \mathrm{~nm}, 24^{\circ} \mathrm{C}\right)$.

Opt. Rot.: $[\alpha]_{\mathrm{D}}^{23} 4.4\left(c=0.98, \mathrm{CHCl}_{3}\right)$ 
Preparation of 2-((4R,5R)-2-(2-Methylphenyl)-4-phenyl-4,5-dihydrooxazol-5yl)methyl)isoindoline-1,3-dione (4d).

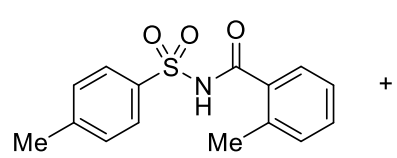

$1 f$ (1.0 equiv)

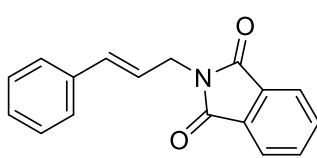

2d (1.2 equiv)

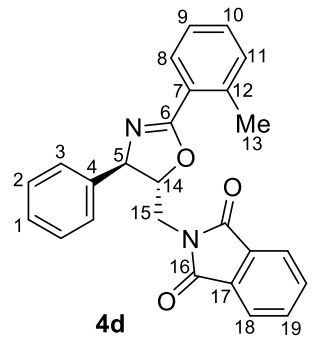

DCE $[0.2 \mathrm{M}], \mathrm{rt}, 24 \mathrm{~h}$

Following General Procedure I, 2-methyl- $N$-tosylbenzamide (289 mg, $1.00 \mathrm{mmol})$, diselenide catalyst $3 \mathbf{a}\left(82.1 \mathrm{mg}, 0.10 \mathrm{mmol}, 0.10\right.$ equiv), 2,4,6- $\mathrm{Me}_{3} \mathrm{PyF}^{+} \mathrm{BF}_{4}^{-}(295 \mathrm{mg}, 1.30 \mathrm{mmol}$, 1.30 equiv), and 2-cinnamylisoindoline-1,3-dione (316 $\mathrm{mg}, 1.20 \mathrm{mmol}, 1.20$ equiv) were combined in 1,2-DCE $(5.0 \mathrm{~mL}, 0.20 \mathrm{M})$ and allowed to stir for $24 \mathrm{~h}$ at $21{ }^{\circ} \mathrm{C}$. The resulting suspension was worked up as described in the General Procedure and purified by column chromatography (150 g silica gel, $5.5 \mathrm{~cm}$ column) eluting with a gradient from hexanes to hexanes/ethyl acetate, 80:20 (50-mL fractions) to yield $368 \mathrm{mg}(93 \%)$ of $\mathbf{4 d}$ as a white solid. A $56.7 \mathrm{mg}$ portion was further purified by Kugelrohr distillation $\left(145^{\circ} \mathrm{C}, 2.6 \times 10^{-5} \mathrm{~mm} \mathrm{Hg}\right)$ to afford $55.0 \mathrm{mg}$ of a clear colorless oil (97\% recovery).

Data for 4d:

m.p. $\quad 87-89^{\circ} \mathrm{C}$ (hexanes)

b.p. $145^{\circ} \mathrm{C}\left(2.6 \times 10^{-5} \mathrm{~mm} \mathrm{Hg}\right)$

${ }^{1} \mathrm{H}$ NMR: $\quad\left(500 \mathrm{MHz}, \mathrm{CDCl}_{3}\right)$

$\delta$ 7.91-7.85 (m, 3 H, HC(8, 18)), 7.75 (dd, $J=5.5,3.0$ Hz, 2 H, HC(19)), 7.38-

$7.32(\mathrm{~m}, 5 \mathrm{H}, \mathrm{HC}(2,3,10)), 7.32-7.23(\mathrm{~m}, 3 \mathrm{H}, \mathrm{HC}(1,9,11)), 5.22(\mathrm{~d}, J=6.7$

$\mathrm{Hz}, 1 \mathrm{H}, \mathrm{HC}(5)), 4.80(\mathrm{td}, J=6.9,4.4 \mathrm{~Hz}, 1 \mathrm{H}, \mathrm{HC}(14)), 4.19$ (dd, $J=14.2,7.2$

$\left.\mathrm{Hz}, 1 \mathrm{H}, \mathrm{H}_{2} \mathrm{C}(15)\right), 4.07\left(\mathrm{dd}, J=14.2,4.4 \mathrm{~Hz}, 1 \mathrm{H}, \mathrm{H}_{2} \mathrm{C}(15)\right), 2.64$ (s, $3 \mathrm{H}$, 
$\left.\mathrm{H}_{3} \mathrm{C}(13)\right)$.

${ }^{13} \mathrm{C} \mathrm{NMR:} \quad\left(126 \mathrm{MHz}, \mathrm{CDCl}_{3}\right)$

$\delta 168.1(\mathrm{C}(16)), 164.2(\mathrm{C}(6), 141.5(\mathrm{C}(4)), 139.2(\mathrm{C}(7)), 134.2(\mathrm{C}(19)), 131.8$ (C(17)), $131.2(\mathrm{C}(11)), 130.8(\mathrm{C}(10)), 130.2(\mathrm{C}(8)), 128.8(\mathrm{C}(2)), 127.7(\mathrm{C}(12))$, $126.5(\mathrm{C}(1)), 126.4(\mathrm{C}(3)), 125.6(\mathrm{C}(9)), 123.5(\mathrm{C}(18)), 83.3(\mathrm{C}(14)), 73.8(\mathrm{C}(5))$, 41.2 (C(15)), 22.0 (C(13)).

IR: (thin-film) 3207 (w), $1774(\mathrm{~m}), 1752$ (s), $1716(\mathrm{~s}), 1643(\mathrm{w}), 1388(\mathrm{~m}), 1306(\mathrm{~m}), 1055(\mathrm{w})$, 715 (m).

HRMS: $\quad\left(\mathrm{ESI}^{+}\right)$Calcd for $\mathrm{C}_{25} \mathrm{H}_{21} \mathrm{~N}_{2} \mathrm{O}_{3}{ }^{+}: 397.1552$, found: 397.1550 .

Analysis: $\quad \mathrm{C}_{25} \mathrm{H}_{20} \mathrm{~N}_{2} \mathrm{O}_{3}$

Calcd: C, 75.74\%; H, 5.09\%; N, 7.07\%

Found: C, 75.53\%, H, 5.04\%; N, 7.13\%

TLC: $\quad R_{f}=0.15(80: 20$ hexanes/ethyl acetate $)\left[\mathrm{UV} / \mathrm{KMnO}_{4}\right]$

CSP-HPLC: $\quad(S, S)-\mathbf{4 d} t_{R}=18.0 \min (1.9 \%),(R, R)-4 \mathbf{d} t_{R}=20.4 \min (98.1 \%)$ (Supelco Astec, hexanes/i-PrOH, 90:10, 1.0mL/min; $\left.220 \mathrm{~nm}, 24{ }^{\circ} \mathrm{C}\right)$.

Opt. Rot.: $[\alpha]_{\mathrm{D}}^{23} 2.6\left(c=0.80, \mathrm{CHCl}_{3}\right)$ 
Preparation of 2-(2-((4R,5R)-2-(2-Methylphenyl)-4-phenyl-4,5-dihydrooxazol-5yl)ethyl)isoindoline-1,3-dione (4e).

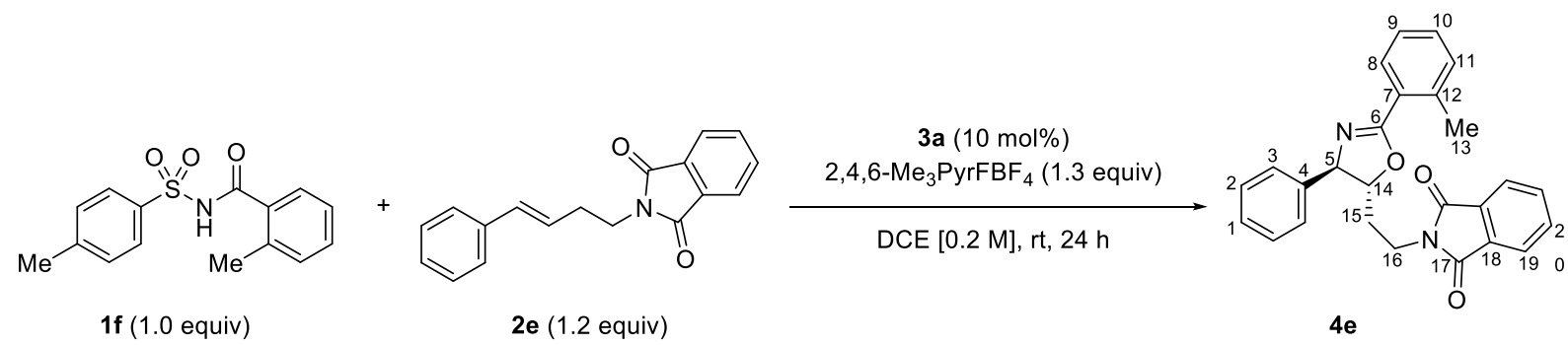

Following General Procedure I, 2-methyl- $N$-tosylbenzamide (289 mg, $1.00 \mathrm{mmol}$ ), diselenide catalyst $3 \mathbf{a}(82.1 \mathrm{mg}, 0.10 \mathrm{mmol}, 0.10$ equiv $), 2,4,6-\mathrm{Me}_{3} \mathrm{PyF}^{+} \mathrm{BF}_{4}^{-}(295 \mathrm{mg}, 1.30 \mathrm{mmol}$, 1.30 equiv), and (E)-2-(4-phenylbut-3-en-1-yl)isoindoline-1,3-dione (333 mg, $1.20 \mathrm{mmol}, 1.20$ equiv) were combined in 1,2-DCE $(5.0 \mathrm{~mL}, 0.20 \mathrm{M})$ (alkene added as a solution in DCE) and allowed to stir for $24 \mathrm{~h}$ at $21{ }^{\circ} \mathrm{C}$. The resulting suspension was worked up as described in the General Procedure and purified by column chromatography (150 g silica gel, $5.5 \mathrm{~cm}$ column) eluting with a gradient from hexanes to hexanes/ethyl acetate, 80:20 (50-mL fractions) to yield $229 \mathrm{mg}(56 \%)$ of $4 \mathrm{e}$ as a white foam. A $57.4 \mathrm{mg}$ portion was further purified by Kugelrohr distillation $\left(145-155^{\circ} \mathrm{C}, 3.4 \times 10^{-5} \mathrm{~mm} \mathrm{Hg}\right)$ to afford $56.4 \mathrm{mg}$ of a clear, glassy solid (98\% recovery).

$\underline{\text { Data for 4e: }}$

b.p. $\quad 145-155^{\circ} \mathrm{C}\left(3.4 \times 10^{-5} \mathrm{~mm} \mathrm{Hg}\right)$

${ }^{1} \mathrm{H}$ NMR: $\quad\left(500 \mathrm{MHz}, \mathrm{CDCl}_{3}\right)$

$\delta 7.85(\mathrm{dd}, J=5.5,3.1 \mathrm{~Hz}, 2 \mathrm{H}, \mathrm{HC}(19)), 7.74-7.70(\mathrm{~m}, 3 \mathrm{H}, \mathrm{HC}(8,20)), 7.37$ (t, $J=7.5 \mathrm{~Hz}, 2 \mathrm{H}, \mathrm{HC}(2)), 7.34-7.28(\mathrm{~m}, 4 \mathrm{H}, \mathrm{HC}(1,3,10)), 7.30(\mathrm{~d}, J=7.6 \mathrm{~Hz}, 1$ H, HC(11)), 7.12 (t, $J=7.6 \mathrm{~Hz}, 1 \mathrm{H}, \mathrm{HC}(9)), 4.99$ (d, $J=7.5 \mathrm{~Hz}, 1 \mathrm{H}, \mathrm{HC}(5))$, 4.53-4.47 (m, $1 \mathrm{H}, \mathrm{HC}(14)), 4.00$ (dt, $\left.J=14.3,7.3 \mathrm{~Hz}, 1 \mathrm{H}, \mathrm{H}_{2} \mathrm{C}(16)\right), 3.93$ (dt, 
$\left.J=13.8,6.9 \mathrm{~Hz}, 1 \mathrm{H}, \mathrm{H}_{2} \mathrm{C}(16)\right), 2.65$ (s, $\left.3 \mathrm{H}, \mathrm{H}_{3} \mathrm{C}(13)\right), 2.31$ (dt, $J=16.2,7.2$

$\left.\mathrm{Hz}, 1 \mathrm{H}, \mathrm{H}_{2} \mathrm{C}(15)\right), 2.27-2.19$ (m, $\left.\mathrm{H}_{2} \mathrm{C}(15)\right)$.

${ }^{13} \mathrm{C} \mathrm{NMR:} \quad\left(126 \mathrm{MHz}, \mathrm{CDCl}_{3}\right)$

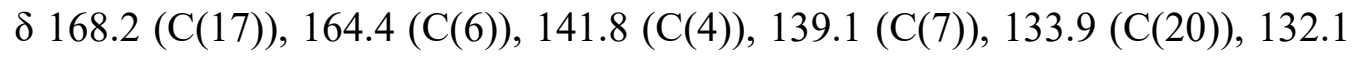

(C(18)), $131.2(\mathrm{C}(11)), 130.6(\mathrm{C}(10)), 130.0(\mathrm{C}(8)), 128.7(\mathrm{C}(2)), 127.7(\mathrm{C}(12))$

$126.7(\mathrm{C}(1)), 126.6(\mathrm{C}(3)), 125.4(\mathrm{C}(9)), 123.2(\mathrm{C}(19)), 84.8(\mathrm{C}(14)), 76.3(\mathrm{C}(5))$,

$35.1(\mathrm{C}(16)), 34.0(\mathrm{C}(15)), 22.0(\mathrm{C}(13))$.

IR: (thin-film)

$1772(w), 1711(\mathrm{~s}), 1641(\mathrm{w}), 1399(\mathrm{w}), 1049(\mathrm{w}), 720(\mathrm{w})$.

HRMS: $\quad\left(\mathrm{ESI}^{+}\right)$Calcd for $\mathrm{C}_{26} \mathrm{H}_{23} \mathrm{~N}_{2} \mathrm{O}_{3}{ }^{+}: 411.1709$, found: 411.1717 .

Analysis: $\quad \mathrm{C}_{26} \mathrm{H}_{22} \mathrm{~N}_{2} \mathrm{O}_{3}$

Calcd: C, 76.08\%; H, 5.40\%; N, 6.82\%

Found: C, 75.83\%; H, 5.37\%; N, 6.94\%

TLC: $\quad R_{f}=0.48(80: 20$ hexanes/ethyl acetate $)\left[\mathrm{UV} / \mathrm{KMnO}_{4}\right]$

CSP-HPLC: $\quad(S, S)-4 \mathrm{e} t_{R}=8.5 \mathrm{~min}(3.4 \%),(R, R)-4 \mathrm{e} t_{R}=10.5 \mathrm{~min}(96.6 \%)$ (Supelco Astec, hexanes/i-PrOH, 70:30, $\left.1.0 \mathrm{~mL} / \mathrm{min} ; 220 \mathrm{~nm}, 24{ }^{\circ} \mathrm{C}\right)$.

Opt. Rot.: $[\alpha]_{\mathrm{D}}^{23} 4.5\left(c=0.71, \mathrm{CHCl}_{3}\right)$ 


\section{Preparation of (4R,5S)-5-(Chloromethyl)-2-(2-methylphenyl)-4-phenyl-4,5-dihydrooxazole}

(4f).
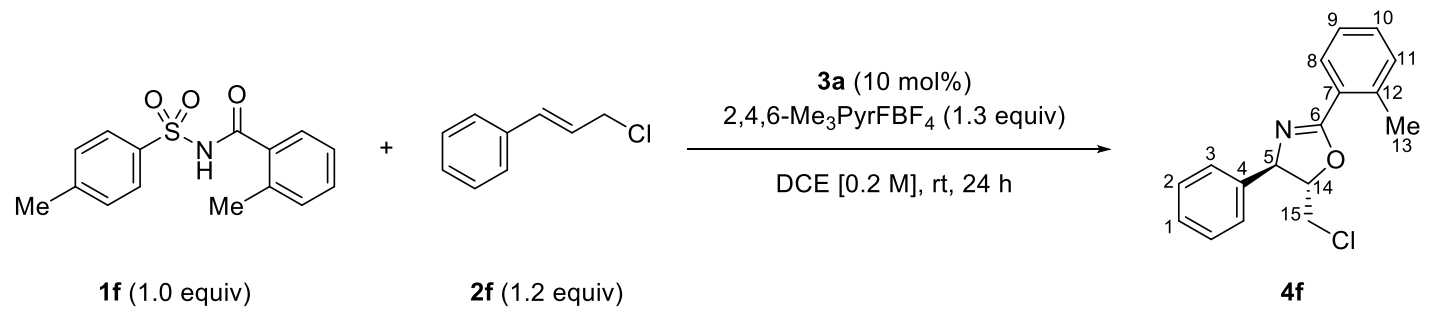

Following General Procedure I, 2-methyl- $N$-tosylbenzamide (289 mg, $1.00 \mathrm{mmol}$ ), diselenide catalyst $3 \mathbf{a}\left(82.1 \mathrm{mg}, 0.10 \mathrm{mmol}, 0.10\right.$ equiv), 2,4,6- $\mathrm{Me}_{3} \mathrm{PyF}^{+} \mathrm{BF}_{4}^{-}(295 \mathrm{mg}, 1.30 \mathrm{mmol}$, 1.30 equiv), and cinnamyl chloride (183 mg, $167 \mu \mathrm{L}, 1.20 \mathrm{mmol}, 1.20$ equiv) were combined in 1,2-DCE (5.0 mL, 0.20 M) (alkene added neat) and allowed to stir for $24 \mathrm{~h}$ at $21^{\circ} \mathrm{C}$. The resulting suspension was worked up as described in the General Procedure and purified by column chromatography (150 g silica gel, $5.5 \mathrm{~cm}$ column) eluting with hexanes/ethyl acetate gradient, 98:2 to $95: 5$ (20-mL fractions) to yield $231 \mathrm{mg}(81 \%)$ of $\mathbf{4 f}$ as a clear oil. A $50.0 \mathrm{mg}$ portion was further purified by Kugelrohr distillation $\left(78^{\circ} \mathrm{C}, 2.9 \times 10^{-5} \mathrm{~mm} \mathrm{Hg}\right)$ to afford $45.2 \mathrm{mg}$ of a clear oil $(90 \%$ recovery).

Data for 4f:

b.p. $78^{\circ} \mathrm{C}\left(2.9 \times 10^{-5} \mathrm{~mm} \mathrm{Hg}\right)$

${ }^{1} \mathrm{H}$ NMR: $\quad\left(500 \mathrm{MHz}, \mathrm{CDCl}_{3}\right)$

$\delta 7.91(\mathrm{dd}, J=7.8,1.5 \mathrm{~Hz}, 1 \mathrm{H}, \mathrm{HC}(8)), 7.41-7.36(\mathrm{~m}, 3 \mathrm{H}, \mathrm{HC}(2,10)), 7.34$

$7.25(\mathrm{~m}, 5 \mathrm{H}, \mathrm{HC}(1,3,9,11)), 5.26(\mathrm{~d}, J=6.1 \mathrm{~Hz}, 1 \mathrm{H}, \mathrm{HC}(5)), 4.66(\mathrm{q}, J=5.5$

$\mathrm{Hz}, 1 \mathrm{H}, \mathrm{HC}(14)), 3.83$ (dd, $\left.J=11.6,5.5 \mathrm{~Hz}, 1 \mathrm{H}, \mathrm{H}_{2} \mathrm{C}(15)\right), 3.80$ (dd, $J=11.6$, $\left.5.1 \mathrm{~Hz}, 1 \mathrm{H}, \mathrm{H}_{2} \mathrm{C}(15)\right), 2.67$ (s, $\left.3 \mathrm{H}, \mathrm{H}_{3} \mathrm{C}(13)\right)$. 
${ }^{13} \mathrm{C} \mathrm{NMR:} \quad\left(126 \mathrm{MHz}, \mathrm{CDCl}_{3}\right)$

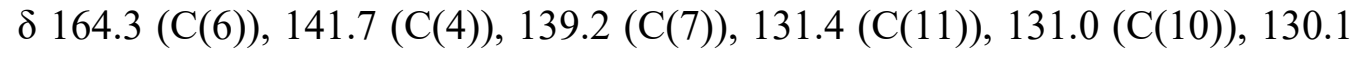
(C(8)), $128.8(\mathrm{C}(2)), 127.8(\mathrm{C}(1)), 126.6(\mathrm{C}(3)), 126.5(\mathrm{C}(12)), 125.7(\mathrm{C}(9)), 84.7$ (C(14)), $73.9(\mathrm{C}(5)), 45.2(\mathrm{C}(15)), 22.0(\mathrm{C}(13))$.

IR: (neat)

$3062(\mathrm{w}), 3029(\mathrm{w}), 2956(\mathrm{w}), 2925(\mathrm{w}), 1642(\mathrm{~s}), 1603(\mathrm{w}), 1574(\mathrm{w}), 1493(\mathrm{~m})$, $1454(\mathrm{~m}), 1427$ (w), 1383 (w), 1340 (w), 1317 (m), $1300(\mathrm{w}), 1268(\mathrm{~m}), 1236$ (w), $1200(\mathrm{w}), 1179(\mathrm{w}), 1162(\mathrm{w}), 1136(\mathrm{w}), 1121(\mathrm{w}), 1071(\mathrm{~m}), 1043(\mathrm{~s}), 1018$ (m), 1002 (m), $977(\mathrm{~m}), 946(\mathrm{w}), 898(\mathrm{w}), 880(\mathrm{w}), 852(\mathrm{w}), 757(\mathrm{~m}), 726(\mathrm{~s})$, $697(\mathrm{~s}), 623(\mathrm{w}), 568(\mathrm{~m}), 546(\mathrm{w}), 522(\mathrm{w}), 466(\mathrm{w})$

HRMS: $\quad\left(\mathrm{ESI}^{+}\right)$Calcd for $\mathrm{C}_{17} \mathrm{H}_{17} \mathrm{ClNO}^{+}: 286.0999$, found: 286.0986

Analysis: $\mathrm{C}_{17} \mathrm{H}_{16} \mathrm{ClNO}$

Calcd: C, 71.45\%; H, 5.64\%; N, 4.90\%

Found: C, 71.12\%; H, 5.61\%; N, 5.18\%

TLC: $\quad R_{f}=0.37(16: 1$, hexanes/ethyl acetate $)[\mathrm{UV}]$

CSP-HPLC: $\quad(R, S)-\mathbf{4 f} t_{R}=5.19 \min (98.0 \%),(S, R)-\mathbf{4 f} t_{R}=6.22 \min (2.0 \%)($ Regis $R, R$-WhelkO1, hexanes/i-PrOH, 70:30, $\left.1.0 \mathrm{~mL} / \mathrm{min} .220 \mathrm{~nm}, 24^{\circ} \mathrm{C}\right)$.

Opt. Rot.: $[\alpha]_{\mathrm{D}}{ }^{23} 32.0\left(c=1.24, \mathrm{CHCl}_{3}\right)$ 
Preparation of $\quad(4 R, 5 S)-5-((B e n z y l o x y) m e t h y l)-2-(2-m e t h y l p h e n y l)-4-p h e n y l-4,5-$ dihydrooxazole (4g).

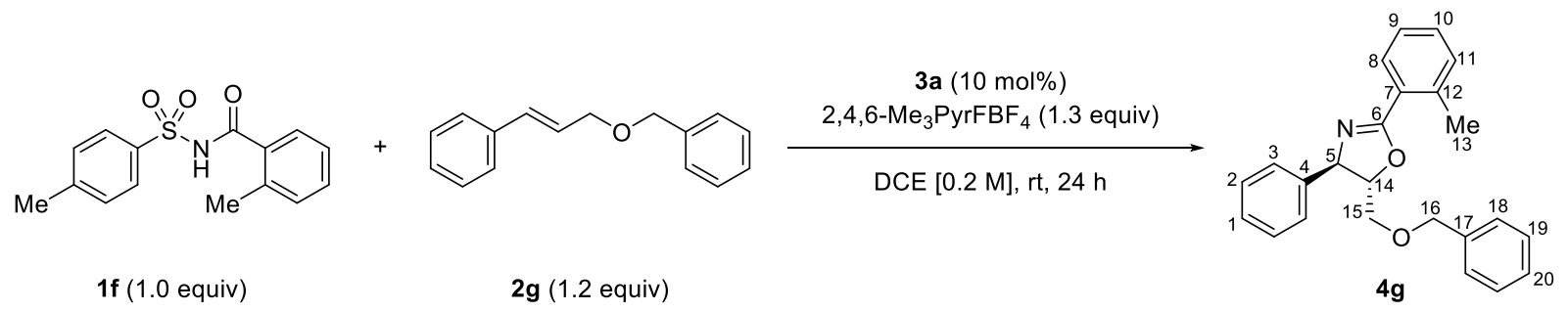

Following General Procedure I, 2-methyl- $N$-tosylbenzamide (289 mg, $1.00 \mathrm{mmol}$ ), diselenide catalyst $\mathbf{3 a}\left(82.1 \mathrm{mg}, 0.10 \mathrm{mmol}, 0.10\right.$ equiv), 2,4,6- $\mathrm{Me}_{3} \mathrm{PyF}^{+} \mathrm{BF}_{4}^{-}$(295 mg, $1.30 \mathrm{mmol}$, 1.30 equiv), and cinnamyl benzyl ether (269 mg, $1.20 \mathrm{mmol}, 1.20$ equiv) were combined in 1,2DCE $(5.0 \mathrm{~mL}, 0.20 \mathrm{M})$ (alkene added as a solution in DCE) and allowed to stir for $24 \mathrm{~h}$ at $21{ }^{\circ} \mathrm{C}$. The resulting suspension was worked up as described in the General Procedure and purified by column chromatography ( $150 \mathrm{~g}$ silica gel, $5.5 \mathrm{~cm}$ column) eluting with a gradient from hexanes to hexanes/ethyl acetate, $88: 12(50-\mathrm{mL}$ fractions) to yield $286 \mathrm{mg}(80 \%)$ of $\mathbf{4 g}$ as a clear colorless oil. A $61.3 \mathrm{mg}$ portion was further purified by Kugelrohr distillation $\left(125-135^{\circ} \mathrm{C}, 4.6 \times 10^{-5} \mathrm{~mm}\right.$ $\mathrm{Hg})$ to afford $60.4 \mathrm{mg}$ of a clear colorless oil (99\% recovery).

\section{Data for $\mathbf{4 g}$ :}

b.p. $\quad 125-135^{\circ} \mathrm{C}\left(4.6 \times 10^{-5} \mathrm{~mm} \mathrm{Hg}\right)$

${ }^{1} \mathrm{H}$ NMR: $\quad\left(500 \mathrm{MHz}, \mathrm{CDCl}_{3}\right)$

$\delta 7.85$ (dd, $J=7.7,1.5 \mathrm{~Hz}, 1 \mathrm{H}, \mathrm{HC}(8)), 7.33-7.29$ (m, $7 \mathrm{H}, \mathrm{HC}(2,11,18,19))$, 7.27-7.18 (m, $6 \mathrm{H}), \mathrm{HC}(1,3,9,10,20)), 5.13$ (d, $J=7.1 \mathrm{~Hz}, 1 \mathrm{H}, \mathrm{HC}(5)), 4.61$ (s, $\left.2 \mathrm{H}, \mathrm{H}_{2} \mathrm{C}(16)\right), 4.56(\mathrm{dt}, J=7.1,4.9 \mathrm{~Hz}, 1 \mathrm{H}, \mathrm{HC}(14)), 3.75$ (dd, $J=10.6,4.5$ $\left.\mathrm{Hz}, 1 \mathrm{H}, \mathrm{H}_{2} \mathrm{C}(15)\right), 3.73\left(\mathrm{dd}, J=10.6,5.4 \mathrm{~Hz}, 1 \mathrm{H}, \mathrm{H}_{2} \mathrm{C}(15)\right), 2.61$ (s, $3 \mathrm{H}$, $\left.\mathrm{H}_{3} \mathrm{C}(13)\right)$. 
${ }^{13} \mathrm{C} \mathrm{NMR:} \quad\left(126 \mathrm{MHz}, \mathrm{CDCl}_{3}\right)$

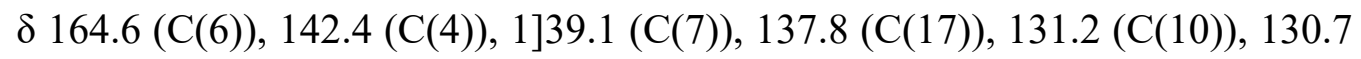

(C(11)), $130.1(\mathrm{C}(8)), 128.7(\mathrm{C}(2)), 128.4(\mathrm{C}(19)), 127.8$ (C(12)), 127.7 (C(18)),

$127.5(\mathrm{C}(20)), 127.0(\mathrm{C}(1)), 126.6(\mathrm{C}(3)), 125.6(\mathrm{C}(9)), 85.2(\mathrm{C}(14)), 73.5$

(C(16)), $72.7(\mathrm{C}(5)), 71.1(\mathrm{C}(15)), 22.0(\mathrm{C}(13))$.

IR: (thin-film)

$3029(\mathrm{w}), 2859$ (w), 1642 (s), 1603 (w), 1494 (m), 1453 (m), 1303 (w), 1275 (m),

$1124(\mathrm{~m}), 1094(\mathrm{~m}), 1046(\mathrm{~s}), 885(\mathrm{w}), 731$ (s), $698(\mathrm{~s})$.

HRMS: $\quad\left(\mathrm{ESI}^{+}\right)$Calcd for $\mathrm{C}_{24} \mathrm{H}_{24} \mathrm{NO}_{2}{ }^{+}: 358.1807$, found: 358.1794 .

Analysis: $\mathrm{C}_{24} \mathrm{H}_{23} \mathrm{NO}_{2}$

Calcd: C, 80.64\%; H, 6.49\%; N, 3.92\%

Found: C, $80.58 \% ; \mathrm{H}, 6.43 \% ; \mathrm{N}, 4.27 \%$

TLC: $\quad R_{f}=0.48(80: 20$ hexanes/ethyl acetate $)\left[\mathrm{UV} / \mathrm{KMnO}_{4}\right]$

CSP-HPLC: $\quad(R, S)-\mathbf{4 g} t_{R}=6.0 \min (95.1 \%),(S, R)-\mathbf{4 g} t_{R}=7.6 \min (4.9 \%)($ Regis $R, R$-WhelkO1, hexanes/i-PrOH, 70:30, $\left.1.0 \mathrm{~mL} / \mathrm{min} ; 220 \mathrm{~nm}, 24^{\circ} \mathrm{C}\right)$.

Opt. Rot.: $[\alpha]_{\mathrm{D}}^{23} 8.9\left(c=1.00, \mathrm{CHCl}_{3}\right)$

\section{Preparation of Methyl 2-((4R,5R)-2-(2-Methylphenyl)-4-phenyl-4,5-dihydrooxazol-5- yl)acetate (4h).}

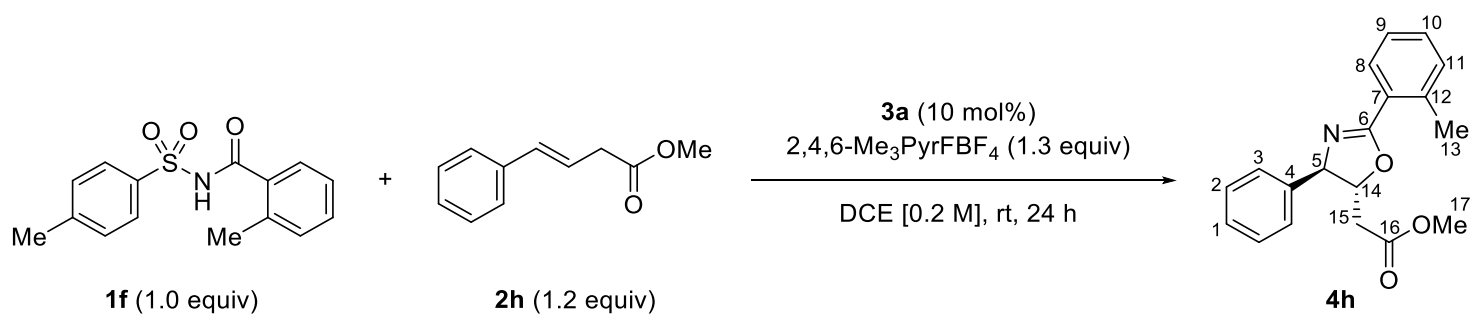

Following General Procedure I, 2-methyl- $N$-tosylbenzamide (289 mg, $1.00 \mathrm{mmol}$ ), 
diselenide catalyst $3 \mathbf{a}\left(82.1 \mathrm{mg}, 0.10 \mathrm{mmol}, 0.10\right.$ equiv), 2,4,6- $\mathrm{Me}_{3} \mathrm{PyF}^{+} \mathrm{BF}_{4}^{-}(295 \mathrm{mg}, 1.30 \mathrm{mmol}$, 1.30 equiv), and methyl (E)-4-phenylbut-3-enoate (212 mg, $1.20 \mathrm{mmol}, 1.20$ equiv) were combined in 1,2-DCE $(5.0 \mathrm{~mL}, 0.20 \mathrm{M})$ (alkene added as a solution in DCE) and allowed to stir for $24 \mathrm{~h}$ at $21^{\circ} \mathrm{C}$. The resulting suspension was worked up as described in the General Procedure and purified by column chromatography (150 g silica gel, $5.5 \mathrm{~cm}$ column) eluting with a gradient from hexanes to hexanes/ethyl acetate, $80: 20$ (50-mL fractions) to yield $254 \mathrm{mg}(82 \%)$ of $\mathbf{4 h}$ as a clear, lightly yellow oil. A $58.3 \mathrm{mg}$ portion was further purified by Kugelrohr distillation (105$110^{\circ} \mathrm{C}, 4.6 \times 10^{-5} \mathrm{~mm} \mathrm{Hg}$ ) to afford $49.7 \mathrm{mg}$ of a clear colorless oil ( $85 \%$ recovery).

\section{Data for 4h:}

b.p. $\quad 105-110^{\circ} \mathrm{C}\left(4.6 \times 10^{-5} \mathrm{~mm} \mathrm{Hg}\right)$

1H NMR: $\quad\left(500 \mathrm{MHz}, \mathrm{CDCl}_{3}\right)$

$\delta 7.82(\mathrm{dd}, J=7.8,1.5 \mathrm{~Hz}, 1 \mathrm{H}, \mathrm{HC}(8)), 7.45-7.23$ (m, $8 \mathrm{H}, \mathrm{HC}(1-3,9-11))$, $4.99(\mathrm{~d}, J=6.4 \mathrm{~Hz}, 1 \mathrm{H}, \mathrm{HC}(5)), 4.78(\mathrm{dt}, J=7.4,5.9 \mathrm{~Hz}, 1 \mathrm{H}, \mathrm{HC}(14)), 3.66$ (s, $\left.3 \mathrm{H}, \mathrm{H}_{3} \mathrm{C}(17)\right), 2.83(\mathrm{dd}, J=15.7,7.5 \mathrm{~Hz}, 1 \mathrm{H}, \mathrm{HC}(15)), 2.75(\mathrm{dd}, J=15.7,5.7$ $\mathrm{Hz}, 1 \mathrm{H}, \mathrm{HC}(15)), 2.57$ (s, $\left.3 \mathrm{H}, \mathrm{H}_{3} \mathrm{C}(13)\right)$.

${ }^{13} \mathrm{C} \mathrm{NMR:} \quad\left(126 \mathrm{MHz}, \mathrm{CDCl}_{3}\right)$

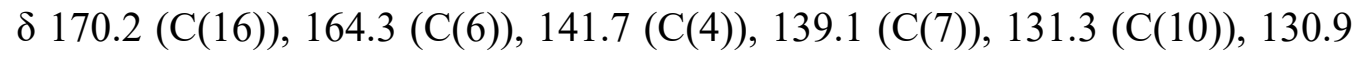
(C(11)), $130.1(\mathrm{C}(8)), 128.7(\mathrm{C}(2)), 127.8$ (C(12)), $126.7(\mathrm{C}(1)), 126.6(\mathrm{C}(3))$, $125.6(\mathrm{C}(9)), 82.3(\mathrm{C}(14)), 75.6(\mathrm{C}(5)), 51.9(\mathrm{C}(17)), 39.8(\mathrm{C}(15)), 21.9(\mathrm{C}(13))$.

IR: (thin-film)

2953 (w), 1739 (s), 1641 (s), 1603 (w), 1494 (w), 1454 (m), 1437 (m), $1384(\mathrm{w})$, 1343 (m), 1233 (m), 1200 (m), 1168 (m), 1048 (s), 990 (m), 887 (w), 759 (m), $729(\mathrm{~m}), 700(\mathrm{~m})$. 
HRMS: $\quad\left(\mathrm{ESI}^{+}\right)$Calcd for $\mathrm{C}_{19} \mathrm{H}_{20} \mathrm{NO}_{3}^{+}: 310.1443$, found: 310.1449 .

Analysis: $\quad \mathrm{C}_{19} \mathrm{H}_{19} \mathrm{NO}_{3}$

Calcd: C, 73.77\%; H, 6.19\%; N, 4.53\%

Found: C, 73.68\%; H, 6.17\%; N, 4.84\%

TLC: $\quad R_{f}=0.29(80: 20$ hexanes/ethyl acetate $)\left[\mathrm{UV} / \mathrm{KMnO}_{4}\right]$

CSP-HPLC: $\quad(R, R)-\mathbf{4 h} t_{R}=7.8 \mathrm{~min}(96.2 \%),(S, S)-\mathbf{4 h} t_{R}=10.8 \mathrm{~min}(3.8 \%)$ (Regis $R, R$-Whelk-

O1, hexanes/i-PrOH, 70:30, $\left.1.0 \mathrm{~mL} / \mathrm{min} ; 220 \mathrm{~nm}, 24^{\circ} \mathrm{C}\right)$.

Opt. Rot.: $[\alpha]_{\mathrm{D}}^{23} 1.8\left(c=1.00, \mathrm{CHCl}_{3}\right)$

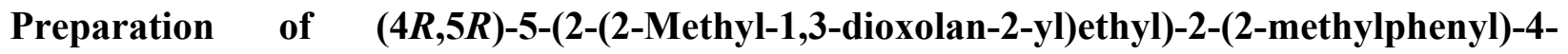
phenyl-4,5-dihydrooxazole (4i).

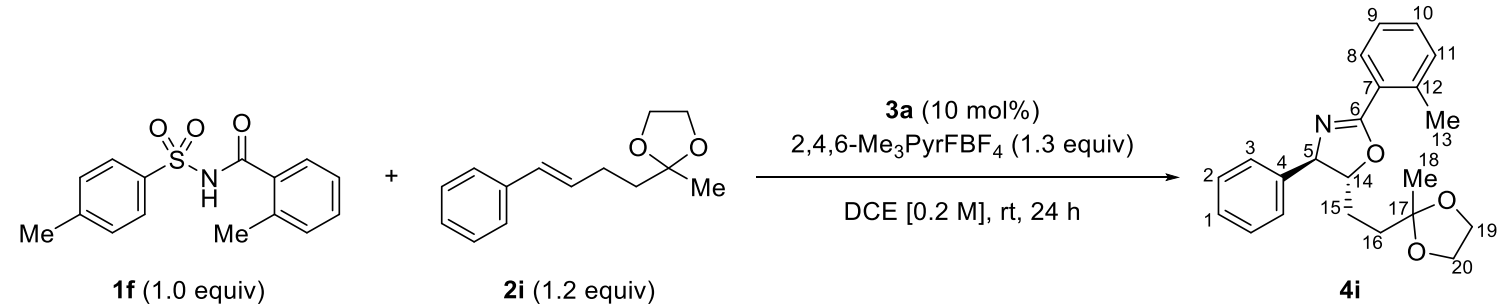

Following General Procedure I, 2-methyl- $N$-tosylbenzamide (289 mg, $1.00 \mathrm{mmol}$ ), diselenide catalyst $3 \mathbf{a}\left(82.1 \mathrm{mg}, 0.10 \mathrm{mmol}, 0.10\right.$ equiv), 2,4,6- $\mathrm{Me}_{3} \mathrm{PyF}^{+} \mathrm{BF}_{4}^{-}(295 \mathrm{mg}, 1.30 \mathrm{mmol}$, 1.30 equiv), and (E)-2-methyl-2-(4-phenylbut-3-en-1-yl)-1,3-dioxolane (262 mg, $1.20 \mathrm{mmol}, 1.20$ equiv) were combined in 1,2-DCE $(5.0 \mathrm{~mL}, 0.20 \mathrm{M})$ (alkene added as a solution in DCE) and allowed to stir for $24 \mathrm{~h}$ at $21{ }^{\circ} \mathrm{C}$. The resulting suspension was worked up as described in the General Procedure and purified by column chromatography (150 g silica gel, $5.5 \mathrm{~cm}$ column) eluting with a gradient from hexanes to hexanes/ethyl acetate, 80:20 (50-mL fractions) to yield $263 \mathrm{mg}(75 \%)$ of $4 \mathbf{i}$ as a clear, lightly yellow oil. A $57.5 \mathrm{mg}$ portion was further purified by Kugelrohr distillation $\left(105^{\circ} \mathrm{C}, 4.4 \times 10^{-5} \mathrm{~mm} \mathrm{Hg}\right)$ to afford $54.9 \mathrm{mg}$ of a clear colorless oil $(95 \%$ 
recovery). Quantitative ${ }^{1} \mathrm{H}$ NMR was conducted as described (page 54 ) with $9.8 \mathrm{mg}$ of $\mathbf{4 i}$ and 3.4 mg of 1,1,2,2-tetrachloroethane (Molar ratio $=1.31$ ) to yield a purity of $95 \%$.

Data for 4i:

b.p. $105^{\circ} \mathrm{C}\left(4.4 \times 10^{-5} \mathrm{~mm} \mathrm{Hg}\right)$

1H NMR: $\quad\left(500 \mathrm{MHz}, \mathrm{CDCl}_{3}\right)$

$\delta 7.85(\mathrm{~d}, J=7.7 \mathrm{~Hz}, 1 \mathrm{H}, \mathrm{HC}(8)), 7.32-7.26(\mathrm{~m}, 3 \mathrm{H}, \mathrm{HC}(2,10)), 7.25-7.16(\mathrm{~m}$, $5 \mathrm{H}, \mathrm{HC}(1,3,9,11)), 4.89(\mathrm{~d}, J=7.0 \mathrm{~Hz}, \mathrm{HC}(5)), 4.40(\mathrm{q}, J=6.9 \mathrm{~Hz}, 1 \mathrm{H}$, $\mathrm{HC}(14)), 3.92-3.82$ (m, $\left.4 \mathrm{H}, \mathrm{H}_{2} \mathrm{C}(19-20)\right), 2.58$ (s, $\left.3 \mathrm{H}, \mathrm{H}_{3} \mathrm{C}(13)\right), 1.93-1.84$ (m, $\left.3 \mathrm{H}, \mathrm{H}_{2} \mathrm{C}(15-16)\right), 1.75$ (tdd, $\left.\left.J=11.2,6.4,2.7 \mathrm{~Hz}, 1 \mathrm{H}\right), \mathrm{H}_{2} \mathrm{C}(15)\right), 1.28$ (s, $3 \mathrm{H}$, $\left.\mathrm{H}_{3} \mathrm{C}(18)\right)$.

${ }^{13} \mathrm{C}$ NMR: $\quad\left(126 \mathrm{MHz}, \mathrm{CDCl}_{3}\right)$

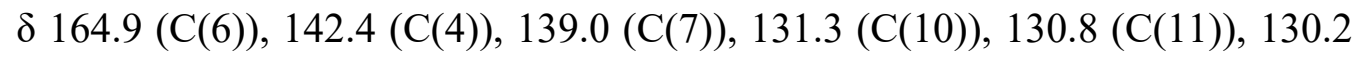
(C(8)), $128.7(\mathrm{C}(2)), 127.6(\mathrm{C} 12)), 127.0(\mathrm{C}(3)), 126.6(\mathrm{C}(3)), 125.6(\mathrm{C}(9))$, $109.5(\mathrm{C}(17)), 87.1(\mathrm{C}(14)), 75.6(\mathrm{C}(5)), 64.7(\mathrm{C}(19$ or 20$)), 64.7(\mathrm{C}(19$ or 20$))$, $34.8(\mathrm{C}(15)), 29.9(\mathrm{C}(16)), 24.0(\mathrm{C}(18)), 22.0(\mathrm{C}(13))$.

IR: (thin-film)

$2955(w), 2880(w), 1639(m), 1603(w), 1494(w), 1453(m), 1377(w), 1307$ (w), $1222(\mathrm{~m}), 1144(\mathrm{w}), 1050(\mathrm{~s}), 948(\mathrm{w}), 900(\mathrm{w}), 866(\mathrm{w}), 759(\mathrm{~m}), 730(\mathrm{~m})$, $700(\mathrm{~m})$.

HRMS: $\quad\left(\mathrm{ESI}^{+}\right.$) Calcd for $\mathrm{C}_{22} \mathrm{H}_{26} \mathrm{NO}_{3}{ }^{+}: 352.1913$, found: 352.1909 .

TLC: $\quad R_{f}=0.21\left(80: 20\right.$ hexanes/ethyl acetate) $\left[\mathrm{UV} / \mathrm{KMnO}_{4}\right]$

CSP-HPLC: $\quad(S, S)-4 \mathbf{i} t_{R}=5.8 \min (2.9 \%),(R, R)-4 \mathbf{i} t_{R}=6.5 \mathrm{~min}(97.1 \%)$ (Supelco Astec, hexanes/i-PrOH, 90:10, $\left.1.0 \mathrm{~mL} / \mathrm{min} ; 220 \mathrm{~nm}, 24^{\circ} \mathrm{C}\right)$. 
Opt. Rot.: $[\alpha]_{\mathrm{D}}^{23} 16.7\left(c=1.00, \mathrm{CHCl}_{3}\right)$

Preparation of (4R,5S)-5-((Benzyloxy)methyl)-2-(2-bromophenyl)-4-phenyl-4,5dihydrooxazole $(4 \mathbf{j})$.

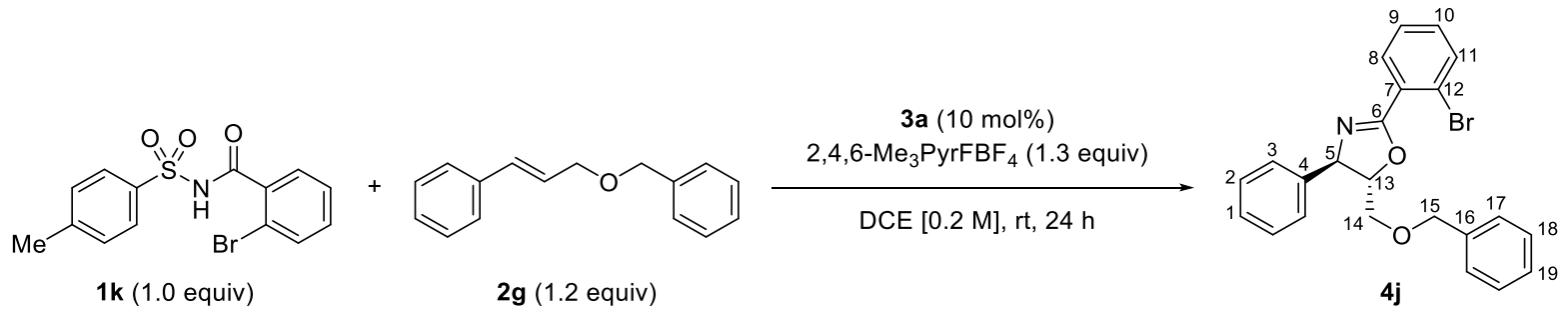

Following General Procedure I, 2-bromo- $N$-tosylbenzamide (354 mg, $1.00 \mathrm{mmol}$ ), diselenide catalyst $3 \mathbf{a}\left(82.1 \mathrm{mg}, 0.10 \mathrm{mmol}, 0.10\right.$ equiv), 2,4,6- $\mathrm{Me}_{3} \mathrm{PyF}^{+} \mathrm{BF}_{4}^{-}(295 \mathrm{mg}, 1.30 \mathrm{mmol}$, 1.30 equiv), and cinnamyl benzyl ether (269 mg, $1.20 \mathrm{mmol}, 1.20$ equiv) were combined in 1,2DCE (5.0 mL, 0.20 M) (alkene added as a solution in DCE) and allowed to stir for $24 \mathrm{~h}$ at $21{ }^{\circ} \mathrm{C}$. The resulting suspension was worked up as described in the General Procedure and purified by column chromatography ( $150 \mathrm{~g}$ silica gel, $5.5 \mathrm{~cm}$ column) eluting with a gradient from hexanes to hexanes/ethyl acetate, 80:20 (50-mL fractions) to yield $370 \mathrm{mg}(88 \%)$ of $\mathbf{4} \mathbf{j}$ as a clear, colorless oil. An $83.8 \mathrm{mg}$ portion was further purified by Kugelrohr distillation $\left(140^{\circ} \mathrm{C}, 3.8 \times 10^{-5} \mathrm{~mm} \mathrm{Hg}\right)$ to afford $79.3 \mathrm{mg}$ of a clear colorless oil ( $95 \%$ recovery).

Data for 4j:

b.p. $140{ }^{\circ} \mathrm{C}\left(3.8 \times 10^{-5} \mathrm{~mm} \mathrm{Hg}\right)$

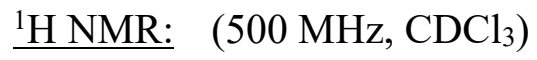

$\delta 7.78(\mathrm{~d}, J=7.7 \mathrm{~Hz}, 1 \mathrm{H}, \mathrm{HC}(8)), 7.66(\mathrm{~d}, J=8.0 \mathrm{~Hz}, 1 \mathrm{H}, \mathrm{HC}(11)), 7.39-7.26$

(m, $12 \mathrm{H}, \mathrm{HC}(1-3,9-10,17-19)), 5.20(\mathrm{~d}, J=7.2 \mathrm{~Hz}, 1 \mathrm{H}, \mathrm{HC}(5)), 4.70$ (dt, $J=$ 7.4, 4.9 Hz, $1 \mathrm{H}, \mathrm{HC}(13)), 4.67$ (d, $\left.J=11.8 \mathrm{~Hz}, 1 \mathrm{H}, \mathrm{H}_{2} \mathrm{C}(15)\right), 4.64$ (d, $J=12.1$ 
$\left.\mathrm{Hz}, 1 \mathrm{H}, \mathrm{H}_{2} \mathrm{C}(15)\right), 3.81\left(\mathrm{~d}, J=3.8 \mathrm{~Hz}, 2 \mathrm{H}, \mathrm{H}_{2} \mathrm{C}(14)\right)$.

${ }^{13} \mathrm{C} \mathrm{NMR:} \quad\left(126 \mathrm{MHz}, \mathrm{CDCl}_{3}\right)$

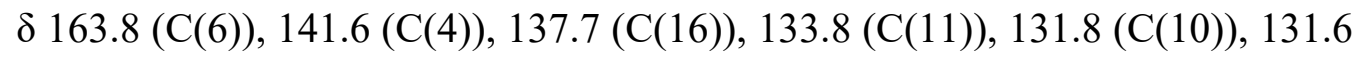
(C(8)), $129.5(\mathrm{C}(7)), 128.7(\mathrm{C}(2)), 128.4(\mathrm{C}(18)), 127.8(\mathrm{C}(9)), 127.7(\mathrm{C}(17))$, 127.7 (C(19)), 127.1 (C(1)), 126.7 (C(3)), 122.0 (C(12)), $86.4(\mathrm{C}(13)), 73.5$ (C(15)), $72.4(\mathrm{C}(5)), 70.7(\mathrm{C}(14))$.

IR: (thin-film)

$3062(\mathrm{w}), 3029(\mathrm{w}), 2859(\mathrm{w}), 1652$ (m), $1590(\mathrm{w}), 1566(\mathrm{w}), 1495(\mathrm{w}), 1474$ (w), $1453(\mathrm{~m}), 1433(\mathrm{w}), 1329(\mathrm{w}), 1301(\mathrm{~m}), 1258(\mathrm{w}), 1127(\mathrm{~m}), 1095(\mathrm{~s}), 1025$

(s), $976(\mathrm{w}), 736(\mathrm{~s}), 698(\mathrm{~s}), 646(\mathrm{w}), 610(\mathrm{w})$.

HRMS: $\quad\left(\mathrm{ESI}^{+}\right)$Calcd for $\mathrm{C}_{23} \mathrm{H}_{21} \mathrm{BrNO}_{2}{ }^{+}$: 422.0756, found: 422.0753 .

Analysis: $\quad \mathrm{C}_{23} \mathrm{H}_{20} \mathrm{BrNO}_{2}$

Calcd: C, 65.41\%; H, 4.77\%; N, 3.32\%

Found: C, 65.33\%; H, 4.68\%; N, 3.68\%

TLC: $\quad R_{f}=0.25(80: 20$ hexanes/ethyl acetate $)\left[\mathrm{UV} / \mathrm{KMnO}_{4}\right]$

CSP-HPLC: $\quad(R, S)-\mathbf{4 j} t_{R}=10.3 \min (6.6 \%),(S, R)-\mathbf{4 j} t_{R}=12.7 \min (93.4 \%)$ (Supelco Astec, hexanes/i-PrOH, 90:10, 1.0mL/min; $\left.220 \mathrm{~nm}, 24{ }^{\circ} \mathrm{C}\right)$.

Opt. Rot.: $[\alpha]_{\mathrm{D}}{ }^{23} 47.7\left(c=0.86, \mathrm{CHCl}_{3}\right)$ 


\section{Preparation of (4R,5R)-2-(2-Methylphenyl)-4,5-dipropyl-4,5-dihydrooxazole (4k).}

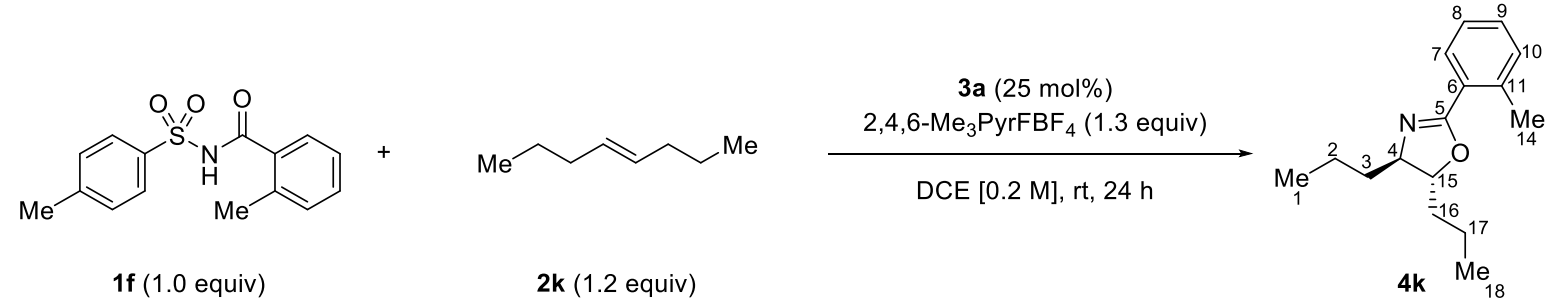

$\underline{3.0 \mathrm{mmol} \text { reaction on racemic starting material for characterization }}$

From a slight modification of General Procedure I, 2-methyl- $N$-tosylbenzamide $(868 \mathrm{mg}$, $3.0 \mathrm{mmol}$ ), diphenyl diselenide (375 mg, $1.2 \mathrm{mmol}, 0.4$ equiv), 2,4,6- $\mathrm{Me}_{3} \mathrm{PyF}^{+} \mathrm{BF}_{4}^{-}(885 \mathrm{mg}, 3.9$ mmol, 1.30 equiv), and trans-4-octene (404 mg, $565 \mu \mathrm{L}, 3.60 \mathrm{mmol}, 1.20$ equiv) were combined in 1,2-DCE $(15.0 \mathrm{~mL}, 0.20 \mathrm{M})$ (alkene added neat) and allowed to stir for $24 \mathrm{~h}$ at $21{ }^{\circ} \mathrm{C}$. The resulting suspension was worked up as described in the General Procedure and purified by column chromatography (50 g silica gel, $3.5 \mathrm{~cm}$ column) eluting with a gradient from hexanes to hexanes/ethyl acetate, 95:5 (25-mL fractions) to yield $214 \mathrm{mg}$. This material was then subjected to preparatory TLC (2000 micron plate) with hexanes/ethyl acetate, 85:15 to afford $171 \mathrm{mg}$. Column chromatography of this material (150 g silica gel, $5.0 \mathrm{~cm}$ column) eluting with a gradient from hexanes to hexanes/ethyl acetate 95:5 to afford $47 \mathrm{mg}$ of a colorless oil. Purification by Kugelrohr distillation $\left(75^{\circ} \mathrm{C}, 6.5 \times 10^{-5} \mathrm{~mm} \mathrm{Hg}\right)$ to afford $39 \mathrm{mg}$ of a clear oil $(5 \%)$.

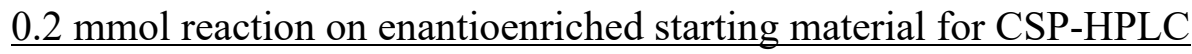

From a slight modification of General Procedure I, 2-methyl- $N$-tosylbenzamide $(58 \mathrm{mg}$, $0.20 \mathrm{mmol}$ ), diselenide catalyst 3a (41 mg, $0.05 \mathrm{mmol}, 0.25$ equiv), 2,4,6- $\mathrm{Me}_{3} \mathrm{PyF}^{+} \mathrm{BF}_{4}^{-}$(59 mg, $0.26 \mathrm{mmol}, 1.30$ equiv), and trans-4-octene ( $27 \mathrm{mg}, 38 \mu \mathrm{L}, 0.24 \mathrm{mmol}, 1.20$ equiv) were combined in 1,2-DCE $(1.0 \mathrm{~mL}, 0.20 \mathrm{M})$ (alkene added neat) and allowed to stir for $24 \mathrm{~h}$ at $21{ }^{\circ} \mathrm{C}$. The resulting suspension was worked up as described in the General Procedure and purified by column chromatography (150 g silica gel, $2.0 \mathrm{~cm}$ column) eluting with chloroform/ethyl acetate, 99:1 (8- 
$\mathrm{mL}$ fractions) to afford $40.1 \mathrm{mg}$. This material was then subjected to preparatory TLC (1000 micron plate) with hexanes/ethyl acetate, $92: 8$ to afford $5.2 \mathrm{mg}$. Column chromatography of this material ( $1 \mathrm{~g}$ silica gel, $0.5 \mathrm{~cm}$ column) eluting with dichloromethane (8-mL fractions) afforded $4.3 \mathrm{mg}$ of a colorless oil ( $9 \%)$.

$\underline{\text { Data for } 4 \mathbf{k}:}$

b.p. $75^{\circ} \mathrm{C}\left(6.5 \times 10^{-5} \mathrm{~mm} \mathrm{Hg}\right)$

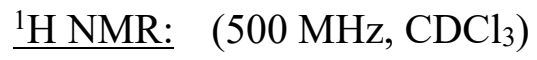

$\delta 7.76(\mathrm{~d}, J=7.8 \mathrm{~Hz}, 1 \mathrm{H}, \mathrm{HC}(7)), 7.30(\mathrm{t}, J=7.5 \mathrm{~Hz}, 1 \mathrm{H}, \mathrm{HC}(9)), 7.23-7.18$ (m, $2 \mathrm{H}, \mathrm{HC}(8,10)), 4.23(\mathrm{q}, J=6.3 \mathrm{~Hz}, 1 \mathrm{H}, \mathrm{HC}(15)), 3.85$ (q, $J=6.3 \mathrm{~Hz}, 1 \mathrm{H}$, HC(4)), 2.57 (s, 3 H, HC(4)), 1.74-1.42 (m, 8 H, H 2 C(2-3, 14-15)), 0.99 (ddd, J $\left.=7.1,6.9,2.6 \mathrm{~Hz}, 6 \mathrm{H}, \mathrm{H}_{3} \mathrm{C}(1,16)\right)$.

${ }^{13} \mathrm{C} \mathrm{NMR:} \quad\left(126 \mathrm{MHz}, \mathrm{CDCl}_{3}\right)$

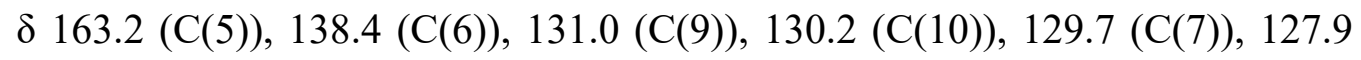
(C11)), 125.4 (C(8)), 84.1 (C(13)), 71.9 (C(4)), 38.3 (C(14)), 37.6 (C(3)), 21.5 (C(12)), $18.9(\mathrm{C}(15)), 18.6(\mathrm{C}(2)), 14.1(\mathrm{C}(16)), 14.0(\mathrm{C}(1))$.

IR: (thin-film) $2958(\mathrm{~m}), 2930(\mathrm{~m}), 2873(\mathrm{w}), 1642(\mathrm{~s}), 1603(\mathrm{w}), 1575(\mathrm{w}), 1493(\mathrm{w}), 1456(\mathrm{~m})$, $1381(\mathrm{w}), 1335(\mathrm{w}), 1320(\mathrm{w}), 1307(\mathrm{w}), 1286(\mathrm{w}), 1261(\mathrm{~m}), 1162(\mathrm{w}), 1138$ (w), $1122(\mathrm{w}), 1070(\mathrm{~m}), 1043(\mathrm{~s}), 984(\mathrm{w}), 966(\mathrm{w}), 937(\mathrm{~m}), 904(\mathrm{w}), 840(\mathrm{w})$, $775(\mathrm{~m}), 727(\mathrm{~s}), 684(\mathrm{w}), 665(\mathrm{w})$.

HRMS: $\quad\left(\mathrm{ESI}^{+}\right)$Calcd for $\mathrm{C}_{16} \mathrm{H}_{24} \mathrm{NO}^{+}: 246.1858$, found: 246.1854 .

TLC: $\quad R_{f}=0.46\left(90: 10\right.$ hexanes/ethyl acetate) $\left[\mathrm{UV} / \mathrm{KMnO}_{4}\right]$

CSP-HPLC: $\quad(S, S)-4 \mathbf{k} t_{R}=6.29 \min (6.8 \%),(R, R)-4 \mathbf{k} t_{R}=9.24 \min (93.2 \%)$ (Supelco Astec, 
hexanes/i-PrOH, 99:1, $\left.1.0 \mathrm{~mL} / \mathrm{min} .220 \mathrm{~nm}, 24^{\circ} \mathrm{C}\right)$.

Preparation of (4R,5R)-5-Methyl-2-(2-methylphenyl)-4-(4-(trifluoromethyl)phenyl)-4,5dihydrooxazole (4I).

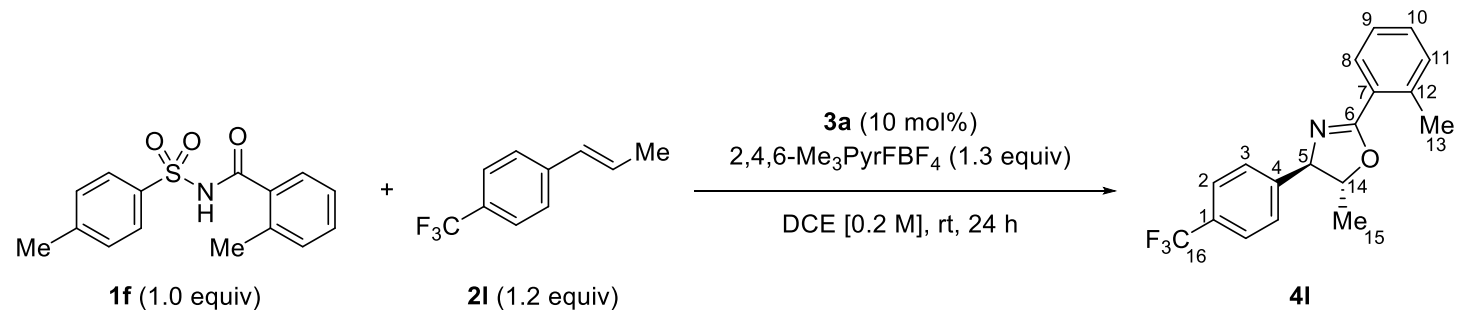

Following General Procedure I, 2-methyl- $N$-tosylbenzamide (289 mg, $1.00 \mathrm{mmol}$ ), diselenide catalyst 3a (82.1 mg, $0.10 \mathrm{mmol}, 0.10$ equiv), 2,4,6- $\mathrm{Me}_{3} \mathrm{PyF}^{+} \mathrm{BF}_{4}^{-}(295 \mathrm{mg}, 1.30 \mathrm{mmol}$, 1.30 equiv), and (E)-1-(prop-1-en-yl)-4-(trifluoromethyl)benzene (223 mg, $1.20 \mathrm{mmol}, 1.20$ equiv) were combined in 1,2-DCE (5.0 mL, $0.20 \mathrm{M})$ (alkene added neat) and allowed to stir for 24 $\mathrm{h}$ at $21{ }^{\circ} \mathrm{C}$. The resulting suspension was worked up as described in the General Procedure and purified by column chromatography ( $150 \mathrm{~g}$ silica gel, $5.5 \mathrm{~cm}$ column) eluting with a hexanes/ethyl acetate gradient, 99:1 to $95: 5$ (50-mL fractions) to yield $194 \mathrm{mg}(61 \%)$ of $\mathbf{4 I}$ as a clear oil. A 50.0 mg portion was further purified by Kugelrohr distillation $\left(65^{\circ} \mathrm{C}, 2.9 \times 10^{-5} \mathrm{~mm} \mathrm{Hg}\right)$ to afford 48.6 mg of a clear oil ( $97 \%$ recovery).

Data for 4l:

b.p. $65^{\circ} \mathrm{C}\left(2.9 \times 10^{-5} \mathrm{~mm} \mathrm{Hg}\right)$

내 NMR: $\quad\left(500 \mathrm{MHz}, \mathrm{CDCl}_{3}\right)$

$\delta 7.89(\mathrm{dd}, J=7.8,1.5 \mathrm{~Hz}, 1 \mathrm{H}, \mathrm{HC}(8)), 7.63(\mathrm{~d}, J=8.1 \mathrm{~Hz}, 2 \mathrm{H}, \mathrm{HC}(2)), 7.44$

(d, $J=8.1 \mathrm{~Hz}, 2 \mathrm{H}, \mathrm{HC}(3)), 7.38$ (td, $J=7.5,1.5 \mathrm{~Hz}, 1 \mathrm{H}, \mathrm{HC}(10)), 7.30-7.24$

(m, $2 \mathrm{H}, \mathrm{HC}(9,11)), 4.96(\mathrm{~d}, J=7.3 \mathrm{~Hz}, 1 \mathrm{H}, \mathrm{HC}(5)), 4.51$ (dq, $J=7.6,6.3 \mathrm{~Hz}$, 
$1 \mathrm{H}, \mathrm{HC}(14)), 2.66$ (s, $\left.3 \mathrm{H}, \mathrm{H}_{3} \mathrm{C}(13)\right), 1.59$ (d, J=6.2 Hz, $\left.3 \mathrm{H}, \mathrm{H}_{3} \mathrm{C}(15)\right)$.

${ }^{13} \mathrm{C}$ NMR: $\quad\left(126 \mathrm{MHz}, \mathrm{CDCl}_{3}\right)$

$\delta 165.2(\mathrm{C}(6)), 146.3(\mathrm{C}(4)), 139.1(\mathrm{C}(7)), 131.4(\mathrm{C}(11)), 130.9(\mathrm{C}(10)), 130.0$ (C(8)), $129.9\left(\mathrm{q},{ }^{2} J_{C-F}=32.4 \mathrm{~Hz}, \mathrm{C}(1)\right), 126.9(\mathrm{C}(12)), 126.8(\mathrm{C}(3)), 125.7$ (q, $\left.{ }^{3} J_{C-F}=3.8 \mathrm{~Hz}, \mathrm{C}(2)\right), 125.7(\mathrm{C}(9)), 124.1\left(\mathrm{q},{ }^{1} J_{C-F}=271.5 \mathrm{~Hz}, \mathrm{C}(16)\right), 83.0$ (C(14)), $77.1(\mathrm{C}(5)), 22.0(\mathrm{C}(13)), 20.9$ (C(15)).

${ }^{19} \mathrm{~F} \mathrm{NMR:} \quad\left(471 \mathrm{MHz}, \mathrm{CDCl}_{3}\right)$

$\delta-62.4$

IR: (neat)

$2974(\mathrm{w}), 2928(\mathrm{w}), 1925(\mathrm{w}), 1637(\mathrm{~m}), 1620(\mathrm{~m}), 1575(\mathrm{w}), 1494(\mathrm{w}), 1456$ (w), $1418(\mathrm{w}), 1382(\mathrm{w}), 1322(\mathrm{~s}), 1278(\mathrm{w}), 1235(\mathrm{w}), 1201(\mathrm{w}), 1162(\mathrm{~m}), 1120$ (s), 1112 (s), 1066 (s), 1046 (s), 1017 (m), 954 (w), 941 (w), 894 (w), 877 (w), $822(\mathrm{~m}), 778(\mathrm{~m}), 766(\mathrm{~m}), 728(\mathrm{~s}), 694(\mathrm{w}), 668(\mathrm{w}), 653(\mathrm{w}), 603(\mathrm{~m}), 560(\mathrm{w})$, $532(\mathrm{w}), 502(\mathrm{w}), 474(\mathrm{w})$

HRMS: $\quad\left(\mathrm{ESI}^{+}\right)$Calcd for $\mathrm{C}_{18} \mathrm{H}_{17} \mathrm{~F}_{3} \mathrm{NO}^{+}:$320.1262, found: 320.1256 .

Analysis: $\mathrm{C}_{18} \mathrm{H}_{16} \mathrm{~F}_{3} \mathrm{NO}$

Calcd: C, 67.70\%; H, 5.05\%; N, 4.39\%

Found: C, 67.64\%; H, 5.14\%; N, 4.53\%

TLC: $\quad R_{f}=0.38(16: 1$ hexanes/ethyl acetate) [UV]

CSP-HPLC: $\quad(R, R)-4 \mathbf{l} t_{R}=12.1 \mathrm{~min}(94.0 \%),(S, S)-4 \mathbf{l} t_{R}=13.9 \min (6.0 \%)$ (Supelco Astec, hexanes/i-PrOH, 98:2, 0.5mL/min; $220 \mathrm{~nm}, 24{ }^{\circ} \mathrm{C}$ )

Opt. Rot.: $[\alpha]_{\mathrm{D}}^{23} 7.0\left(c=1.15, \mathrm{CHCl}_{3}\right)$ 
Preparation of $\quad(4 R, 5 R)-4-(4-M e t h o x y p h e n y l)-5-m e t h y l-2-(2-m e t h y l p h e n y l)-4,5-$ dihydrooxazole $(4 \mathrm{~m})$.

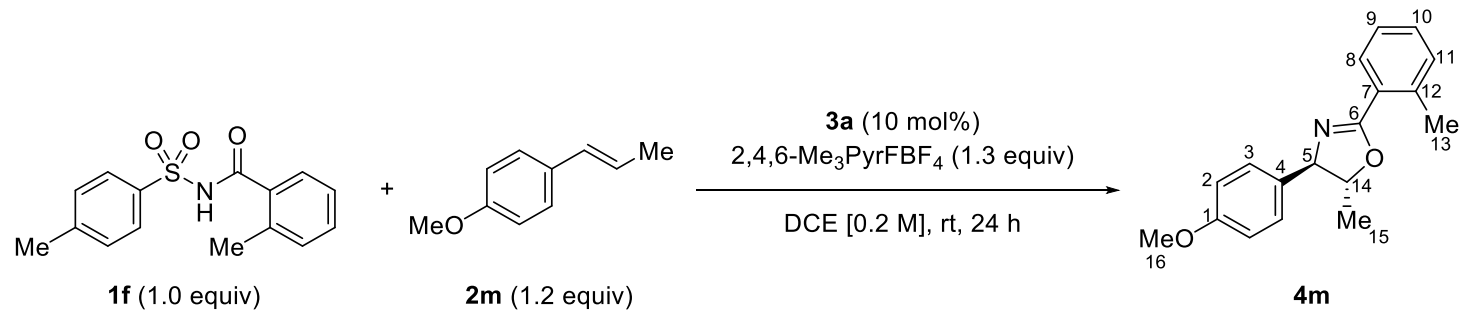

Following General Procedure I, 2-methyl- $N$-tosylbenzamide (289 mg, $1.00 \mathrm{mmol}$ ), diselenide catalyst $\mathbf{3 a}(82.1 \mathrm{mg}, 0.10 \mathrm{mmol}, 0.10$ equiv $), 2,4,6-\mathrm{Me}_{3} \mathrm{PyF}^{+} \mathrm{BF}_{4}^{-}(295 \mathrm{mg}, 1.30 \mathrm{mmol}$, 1.30 equiv), and trans-anethole (178 mg, $178 \mu \mathrm{L}, 1.20 \mathrm{mmol}, 1.20$ equiv) were combined in 1,2DCE $(5.0 \mathrm{~mL}, 0.20 \mathrm{M})$ (alkene added neat) and allowed to stir for $24 \mathrm{~h}$ at $21{ }^{\circ} \mathrm{C}$. The resulting suspension was worked up as described in the General Procedure and purified by column chromatography (150 g silica gel, $5.5 \mathrm{~cm}$ column) eluting a hexanes/ethyl acetate gradient, 98:2 to 92:8 (20-mL fractions) to yield $189 \mathrm{mg}(67 \%)$ of $\mathbf{4 m}$ as a yellow oil. A $50.1 \mathrm{mg}$ portion was further purified by Kugelrohr distillation $\left(94{ }^{\circ} \mathrm{C}, 3.0 \times 10^{-5} \mathrm{~mm} \mathrm{Hg}\right)$ to afford $46.2 \mathrm{mg}$ of a clear oil (92\% recovery).

Data for 4m:

b.p. $94{ }^{\circ} \mathrm{C}\left(3.0 \times 10^{-5} \mathrm{~mm} \mathrm{Hg}\right)$

${ }^{1} \mathrm{H}$ NMR: $\quad\left(500 \mathrm{MHz}, \mathrm{CDCl}_{3}\right)$

$\delta 7.88(\mathrm{dd}, J=7.8,1.4 \mathrm{~Hz}, 1 \mathrm{H}, \mathrm{HC}(8)), 7.36(\mathrm{td}, J=7.5,1.5 \mathrm{~Hz}, 1 \mathrm{H}, \mathrm{HC}(10))$, 7.27-7.21 (m, $2 \mathrm{H}, \mathrm{HC}(9,11)), 7.23$ (d, $J=8.7 \mathrm{~Hz}, 2 \mathrm{H}, \mathrm{HC}(3)), 6.90$ (d, $J=8.6$ $\mathrm{Hz}, 2 \mathrm{H}, \mathrm{HC}(2)), 4.84$ (d, $J=7.3 \mathrm{~Hz}, 1 \mathrm{H}, \mathrm{HC}(5)), 4.50$ (pent, $J=6.5 \mathrm{~Hz}, 1 \mathrm{H}$, $\mathrm{HC}(14)), 3.81$ (s, $\left.3 \mathrm{H}, \mathrm{H}_{3} \mathrm{C}(16)\right), 2.65$ (s, $\left.3 \mathrm{H}, \mathrm{H}_{3} \mathrm{C}(13)\right), 1.54$ (d, J = 6.2 Hz, $3 \mathrm{H}$, $\left.\mathrm{H}_{3} \mathrm{C}(15)\right)$. 
${ }^{13} \mathrm{C} \mathrm{NMR:} \quad\left(126 \mathrm{MHz}, \mathrm{CDCl}_{3}\right)$

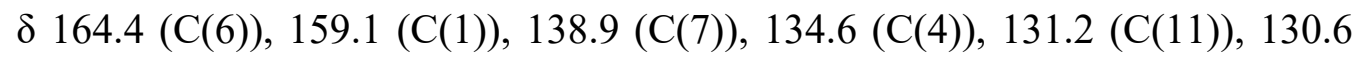
(C(10)), $130.0(\mathrm{C}(8)), 127.6(\mathrm{C}(3)), 127.4(\mathrm{C}(12)), 125.6(\mathrm{C}(9)), 114.1(\mathrm{C}(2))$, $83.4(\mathrm{C}(14)), 77.2(\mathrm{C}(5)), 55.3(\mathrm{C}(16)), 21.9(\mathrm{C}(13)), 20.8(\mathrm{C}(15))$.

IR: (neat) $2969(\mathrm{w}), 2927(\mathrm{w}), 2835(\mathrm{w}), 1636(\mathrm{~m}), 1613(\mathrm{~m}), 1584(\mathrm{w}), 1511(\mathrm{~s}), 1455(\mathrm{~m})$, 1442 (m), $1381(\mathrm{w}), 1337(\mathrm{w}), 1302$ (m), $1277(\mathrm{~m}), 1244(\mathrm{~s}), 1174(\mathrm{~m}), 1138$ (w), $1113(\mathrm{w}), 1041(\mathrm{~s}), 1002(\mathrm{w}), 946(\mathrm{w}), 892(\mathrm{w}), 875(\mathrm{~m}), 851(\mathrm{w}), 825(\mathrm{~m})$, $772(\mathrm{~m}), 728(\mathrm{~s}), 677(\mathrm{w}), 585(\mathrm{w}), 561(\mathrm{w}), 544(\mathrm{w})$

HRMS: $\quad\left(\mathrm{ESI}^{+}\right)$Calcd for $\mathrm{C}_{18} \mathrm{H}_{20} \mathrm{NO}_{2}^{+}:$282.1494, found: 282.1494.

Analysis: $\mathrm{C}_{18} \mathrm{H}_{19} \mathrm{NO}_{2}$

Calcd: C, 76.84\%; H, 6.81\%; N, 4.98\%

Found: C, 76.66\%; H, 6.79\%; N, 5.11\%

TLC: $\quad R_{f}=0.26(8: 1$ hexanes/ethyl acetate $)[\mathrm{UV}]$

CSP-HPLC: $\quad(R, R)-\mathbf{4 m} t_{R}=6.56 \min (86.4 \%),(S, S)-4 m t_{R}=10.30 \min (13.6 \%)($ Regis $R, R-$ Whelk-O1, hexanes/i-PrOH, 70:30, $\left.1.0 \mathrm{~mL} / \mathrm{min} .220 \mathrm{~nm}, 24^{\circ} \mathrm{C}\right)$.

Opt. Rot.: $[\alpha]_{\mathrm{D}}^{23} 24.1\left(c=0.47, \mathrm{CHCl}_{3}\right)$ 
Preparation of $\quad(4 R, 5 R)-4-(2-B r o m o p h e n y l)-5-m e t h y l-2-(2-m e t h y l p h e n y l)-4,5-$ dihydrooxazole (4n).

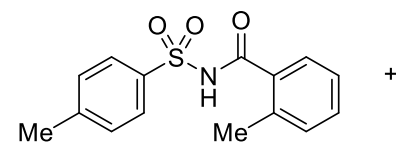

1f (1.0 equiv)

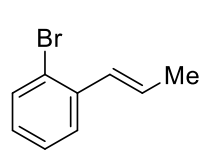

2n (1.2 equiv)

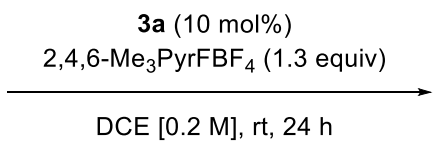

DCE [0.2 M], rt, $24 \mathrm{~h}$

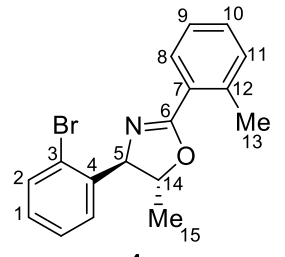

$4 n$

Following General Procedure I, 2-methyl- $N$-tosylbenzamide (289 mg, $1.00 \mathrm{mmol}$ ), diselenide catalyst $3 \mathbf{a}\left(82.1 \mathrm{mg}, 0.10 \mathrm{mmol}, 0.10\right.$ equiv), 2,4,6- $\mathrm{Me}_{3} \mathrm{PyF}^{+} \mathrm{BF}_{4}^{-}(295 \mathrm{mg}, 1.30 \mathrm{mmol}$, 1.30 equiv), and (E)-1-bromo-2-(prop-1-en-1-yl)benzene (223 mg, $1.20 \mathrm{mmol}, 1.20$ equiv) were combined in 1,2-DCE $(5.0 \mathrm{~mL}, 0.20 \mathrm{M})$ (alkene added neat) and allowed to stir for $24 \mathrm{~h}$ at $21{ }^{\circ} \mathrm{C}$. The resulting suspension was worked up as described in the General Procedure and purified by column chromatography (150 g silica gel, $5.5 \mathrm{~cm}$ column) eluting a chloroform/ethyl acetate gradient from pure chloroform to pure ethyl acetate $(20-\mathrm{mL}$ fractions) to yield $176 \mathrm{mg}(53 \%)$ of 4n as a clear oil. A $102.8 \mathrm{mg}$ portion was further purified by Kugelrohr distillation $\left(89^{\circ} \mathrm{C}, 3.0 \mathrm{x}\right.$ $10^{-5} \mathrm{~mm} \mathrm{Hg}$ ) to afford $97.7 \mathrm{mg}$ of a clear oil (95\% recovery).

$\underline{\text { Data for 4n: }}$

b.p. $89^{\circ} \mathrm{C}\left(3.0 \times 10^{-5} \mathrm{~mm} \mathrm{Hg}\right)$

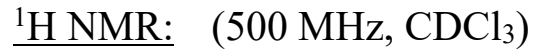

$\delta 7.93(\mathrm{dd}, J=7.7,1.5 \mathrm{~Hz}, 1 \mathrm{H}, \mathrm{HC}(8)), 7.58(\mathrm{dd}, J=7.9,1.1 \mathrm{~Hz}, 1 \mathrm{H}, \mathrm{HC}(2))$, $7.39(\mathrm{td}, J=7.5,1.5 \mathrm{~Hz}, 1 \mathrm{H}, \mathrm{HC}(10)), 7.32-7.25(\mathrm{~m}, 4 \mathrm{H}, \mathrm{HC}(3,9,11,16,17))$, 7.14 (ddd, $J=8.0,6.7,2.3 \mathrm{~Hz}, 1 \mathrm{H}, \mathrm{HC}(1)), 5.37$ (d, $J=5.5 \mathrm{~Hz}, 1 \mathrm{H}, \mathrm{HC}(5))$, 4.52 (pent, $J=6.2 \mathrm{~Hz}, 1 \mathrm{H}, \mathrm{HC}(14)), 2.69$ (s, $\left.3 \mathrm{H}, \mathrm{H}_{3} \mathrm{C}(13)\right), 1.66$ (d, $J=6.3 \mathrm{~Hz}$, $\left.3 \mathrm{H}, \mathrm{H}_{3} \mathrm{C}(15)\right)$. 
${ }^{13} \mathrm{C} \mathrm{NMR:} \quad\left(126 \mathrm{MHz}, \mathrm{CDCl}_{3}\right)$

$\delta 165.6(\mathrm{C}(6)), 141.5(\mathrm{C}(4)), 139.1(\mathrm{C}(7)), 132.8(\mathrm{C}(2)), 131.4(\mathrm{C}(11)), 130.8$ (C(10)), $130.1(\mathrm{C}(8)), 128.9(\mathrm{C}(16)), 128.2(\mathrm{C}(1)), 127.8(\mathrm{C}(17)), 127.1(\mathrm{C}(12))$, $125.7(\mathrm{C}(9)), 122.9(\mathrm{C}(3)), 82.9(\mathrm{C}(14)), 75.6(\mathrm{C}(5)), 22.1(\mathrm{C}(13)), 21.3(\mathrm{C}(15))$.

IR: (neat)

$3062(\mathrm{w}), 2975(\mathrm{w}), 2926(\mathrm{w}), 1925(\mathrm{w}), 1635(\mathrm{~s}), 1601(\mathrm{w}), 1566(\mathrm{w}), 1492(\mathrm{w})$, 1465 (m), 1455 (m), 1439 (m), 1375 (w), 1329 (m), 1303 (w), 1276 (m), 1254 (w), $1233(\mathrm{~m}), 1198(\mathrm{w}), 1161(\mathrm{w}), 1138(\mathrm{w}), 1120(\mathrm{w}), 1083(\mathrm{w}), 1042(\mathrm{~s}), 1004$ (m), $948(\mathrm{w}), 892(\mathrm{w}), 876(\mathrm{~m}), 828(\mathrm{w}), 777(\mathrm{~m}), 747$ (s), $726(\mathrm{~s}), 690(\mathrm{~m}), 671$ (m), $625(\mathrm{w}), 563(\mathrm{w}), 544(\mathrm{w}), 504(\mathrm{w}), 490(\mathrm{w})$

HRMS: $\quad\left(\mathrm{ESI}^{+}\right)$Calcd for $\mathrm{C}_{17} \mathrm{H}_{17} \mathrm{BrNO}^{+}:$330.0494, found: 330.0496 .

Analysis: $\mathrm{C}_{17} \mathrm{H}_{16} \mathrm{BrNO}$

Calcd: C, 61.83\%; H, 4.88\%; N, 4.24\%

Found: $61.92 \% ; H, 4.79 \% ; \mathrm{N}, 4.48 \%$

TLC: $\quad R_{f}=0.21(16: 1$, hexanes/ethyl acetate $)[\mathrm{UV}]$

CSP-HPLC: $\quad(R, R)-4 \mathbf{n} t_{R}=6.46 \min (97.2 \%),(S, S)-4 \mathbf{n} t_{R}=8.25 \min (2.85 \%)($ Regis $R, R-$ Whelk-O1, hexanes/i-PrOH, 95:5, $\left.1.0 \mathrm{~mL} / \mathrm{min} .220 \mathrm{~nm}, 24^{\circ} \mathrm{C}\right)$.

Opt. Rot.: $[\alpha]_{\mathrm{D}}^{23}-84.6\left(c=1.40, \mathrm{CHCl}_{3}\right)$ 
Preparation of $(4 R, 5 S)-5-((B e n z y l o x y) m e t h y l)-2-(2-m e t h y l p h e n y l)-4-(t h i o p h e n-3-y l)-4,5-$ dihydrooxazole (4o).
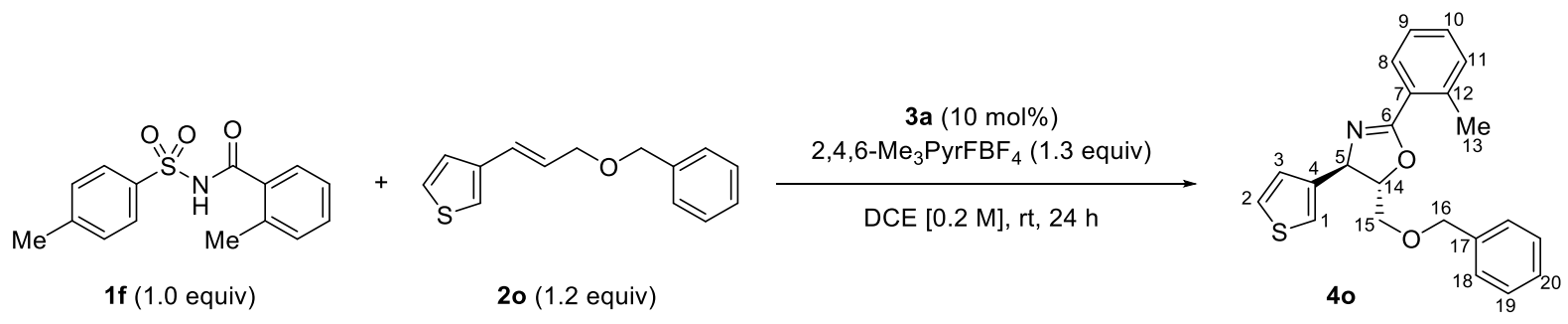

Following General Procedure I, 2-methyl- $N$-tosylbenzamide (289 mg, $1.00 \mathrm{mmol}$ ), diselenide catalyst 3a $(82.1 \mathrm{mg}, 0.10 \mathrm{mmol}, 0.10$ equiv $), 2,4,6-\mathrm{Me}_{3} \mathrm{PyF}^{+} \mathrm{BF}_{4}^{-}(295 \mathrm{mg}, 1.30 \mathrm{mmol}$, 1.30 equiv), and (E)-3-(3-benzyloxy)prop-1-en-1-yl)thiophene (276 mg, $1.20 \mathrm{mmol}, 1.20$ equiv) were combined in 1,2-DCE $(5.0 \mathrm{~mL}, 0.20 \mathrm{M})$ (alkene added as a solution in DCE) and allowed to stir for $24 \mathrm{~h}$ at $21^{\circ} \mathrm{C}$. The resulting suspension was worked up as described in the General Procedure and purified by column chromatography (150 g silica gel, $5.5 \mathrm{~cm}$ column) eluting with a gradient from hexanes to hexanes/ethyl acetate, 89:11 (50-mL fractions) to yield $192 \mathrm{mg}(53 \%)$ of $4 \mathrm{o}$ as a clear, colorless oil. A $64.0 \mathrm{mg}$ portion was further purified by Kugelrohr distillation $\left(110-120^{\circ} \mathrm{C}, 4.4 \times 10^{-5} \mathrm{~mm} \mathrm{Hg}\right)$ to afford $62.4 \mathrm{mg}$ of a clear colorless oil (98\% recovery). Quantitative ${ }^{1} \mathrm{H}$ NMR was conducted as described (page 54 ) with $11.9 \mathrm{mg}$ of $\mathbf{4 0}$ and $5.7 \mathrm{mg}$ of 1,1,2,2-tetrachloroethane (Molar ratio $=0.93$ ) to yield a purity of $97 \%$.

Data for 4o:

b.p. $\quad 110-120^{\circ} \mathrm{C}\left(4.4 \times 10^{-5} \mathrm{~mm} \mathrm{Hg}\right)$

${ }^{1} \mathrm{H}$ NMR: $\quad\left(500 \mathrm{MHz}, \mathrm{CDCl}_{3}\right)$

$\delta 7.87(\mathrm{dd}, J=7.8,1.5 \mathrm{~Hz}, 1 \mathrm{H}, \mathrm{HC}(8)), 7.37-7.32(\mathrm{~m}, 5 \mathrm{H}, \mathrm{HC}(2,11,19,20))$, 7.32-7.27 (m, 2 H, HC(18)), 7.27-7.22 (m, 2 H, HC(9, 10)), 7.20-7.18 (m, $1 \mathrm{H}$, $\mathrm{HC}(1)), 7.03$ (dd, $J=4.9,1.3 \mathrm{~Hz}, 1 \mathrm{H}, \mathrm{HC}(3)), 5.26$ (d, $J=7.0 \mathrm{~Hz}, 1 \mathrm{H}, \mathrm{HC}(5))$, 
4.65-4.60 (m, $1 \mathrm{H}, \mathrm{HC}(14)), 4.64$ (s, $\left.2 \mathrm{H}, \mathrm{H}_{2} \mathrm{C}(16)\right), 3.77$ (d, $J=5.0 \mathrm{~Hz}, 2 \mathrm{H}$, $\left.\mathrm{H}_{2} \mathrm{C}(15)\right), 2.64\left(\mathrm{~s}, 3 \mathrm{H}, \mathrm{H}_{3} \mathrm{C}(13)\right)$.

${ }^{13} \mathrm{C} \mathrm{NMR:} \quad\left(126 \mathrm{MHz}, \mathrm{CDCl}_{3}\right)$

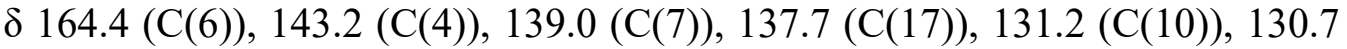

(C(11)), $130.0(\mathrm{C}(8)), 128.4(\mathrm{C}(19)), 127.8$ (C(20)), 127.7 (C(18)), 126.9

(C(12)), $126.5(\mathrm{C}(2)), 125.9(\mathrm{C}(3)), 125.6(\mathrm{C}(9)), 121.1(\mathrm{C}(1)), 84.3(\mathrm{C}(14)), 73.5$

(C(16)), 70.9 (C(15)), $68.8(\mathrm{C}(5)), 21.9$ (C(13)).

IR: (thin-film)

$2860(\mathrm{w}), 1642(\mathrm{~s}), 1495(\mathrm{w}), 1454(\mathrm{~m}), 1275(\mathrm{~m}), 1124(\mathrm{~m}), 1046(\mathrm{~s}), 839(\mathrm{w})$,

$781(\mathrm{~m}), 736(\mathrm{~s}), 698(\mathrm{~m})$.

HRMS: $\quad\left(\mathrm{ESI}^{+}\right)$Calcd for $\mathrm{C}_{22} \mathrm{H}_{22} \mathrm{NO}_{2} \mathrm{~S}^{+}: 364.1371$, found: 364.1371 .

TLC: $\quad R_{f}=0.16(90: 10$ hexanes/ethyl acetate $)\left[\mathrm{UV} / \mathrm{KMnO}_{4}\right]$

CSP-HPLC: $\quad(S, R)-40 \quad t_{R}=7.2 \mathrm{~min}(7.3 \%),(R, S)-40 t_{R}=9.3 \mathrm{~min}(92.7 \%)$ (Supelco Astec, hexanes/i-PrOH, 90:10, 1.0mL/min; $\left.220 \mathrm{~nm}, 24{ }^{\circ} \mathrm{C}\right)$.

Opt. Rot.: $[\alpha]_{\mathrm{D}}^{23} 30.2\left(c=1.00\right.$ in $\left.\mathrm{CHCl}_{3}\right)$

Preparation of (4R,5S)-5-((Benzyloxy)methyl)-2-(2-methylphenyl)-4-(naphthalen-2-yl)-4,5dihydrooxazole (4p).

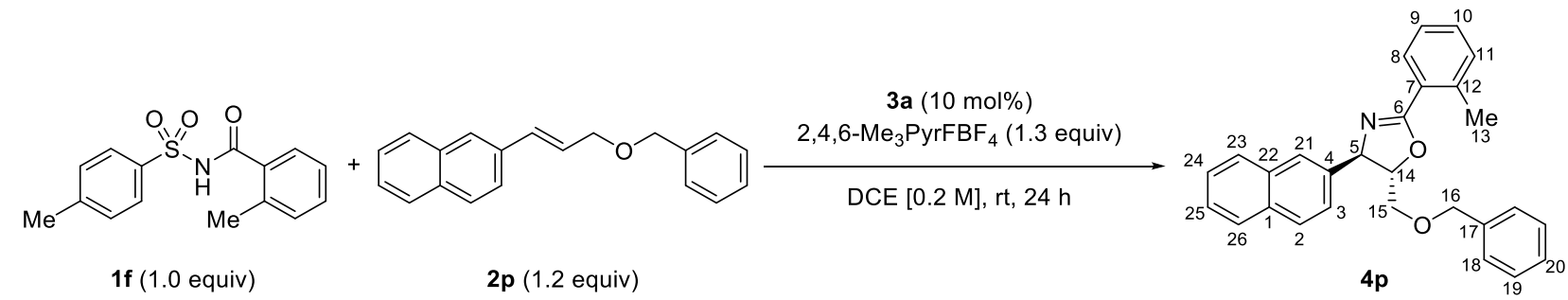

Following General Procedure I, 2-methyl- $N$-tosylbenzamide (289 mg, $1.00 \mathrm{mmol}$ ), diselenide catalyst $\mathbf{3 a}\left(82.1 \mathrm{mg}, 0.10 \mathrm{mmol}, 0.10\right.$ equiv), 2,4,6- $\mathrm{Me}_{3} \mathrm{PyF}^{+} \mathrm{BF}_{4}^{-}$(295 mg, $1.30 \mathrm{mmol}$, 
1.30 equiv), and (E)-2-(3-(benzyloxy)prop-1-en-1-yl)naphthalene (329 mg, $1.20 \mathrm{mmol}, 1.20$ equiv) were combined in 1,2-DCE $(5.0 \mathrm{~mL}, 0.20 \mathrm{M})$ (alkene added as a solution in DCE) and allowed to stir for $24 \mathrm{~h}$ at $21{ }^{\circ} \mathrm{C}$. The resulting suspension was worked up as described in the General Procedure and purified by column chromatography (150 g silica gel, $5.5 \mathrm{~cm}$ column) eluting with a gradient from hexanes to hexanes/ethyl acetate, 90:10 (50-mL fractions) to yield $326 \mathrm{mg}(80 \%)$ of $4 \mathbf{p}$ as a clear, colorless oil. A $56.1 \mathrm{mg}$ portion was further purified by Kugelrohr distillation $\left(120-145{ }^{\circ} \mathrm{C}, 4.0 \times 10^{-5} \mathrm{~mm} \mathrm{Hg}\right)$ to afford $54.8 \mathrm{mg}$ of a clear colorless oil $(98 \%$ recovery).

Data for $\mathbf{4 p}$ :

b.p. $\quad 120-145^{\circ} \mathrm{C}\left(4.0 \times 10^{-5} \mathrm{~mm} \mathrm{Hg}\right)$

1H NMR: $\left(500 \mathrm{MHz}, \mathrm{CDCl}_{3}\right)$

$\delta 7.97(\mathrm{dd}, J=7.7,1.5 \mathrm{~Hz}, 1 \mathrm{H}, \mathrm{HC}(8))$, 7.87-7.80 (m, $3 \mathrm{H}, \mathrm{HC}(19,20)), 7.77$ (d, $J=1.8 \mathrm{~Hz}, 1 \mathrm{H}, \mathrm{HC}(21)), 7.50-7.45$ (m, $2 \mathrm{H}, \mathrm{HC}(24,25)), 7.44-7.35$ (m, 6 $\mathrm{H}, \mathrm{HC}(2,3,10,18,23,26)), 5.38(\mathrm{~d}, J=7.1 \mathrm{~Hz}, 1 \mathrm{H}, \mathrm{HC}(5)), 4.72-4.65$ (m, 3 H, $\left.\mathrm{HC}(14), \mathrm{H}_{2} \mathrm{C}(16)\right), 3.86$ (dd, $\left.J=10.6,4.5 \mathrm{~Hz}, 1 \mathrm{H}, \mathrm{H}_{2} \mathrm{C}(15)\right), 3.84$ (dd, $J=$ 10.6, $\left.5.3 \mathrm{~Hz}, 1 \mathrm{H}, \mathrm{H}_{2} \mathrm{C}(15)\right), 2.70$ (s, $\left.3 \mathrm{H}, \mathrm{H}_{3} \mathrm{C}(13)\right)$.

${ }^{13} \mathrm{C} \mathrm{NMR}: \quad\left(126 \mathrm{MHz}, \mathrm{CDCl}_{3}\right)$

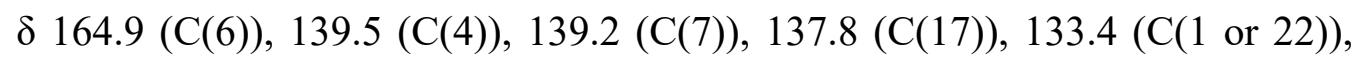
$132.9(\mathrm{C}(1$ or 22$)), 131.3(\mathrm{C}(11)), 130.8(\mathrm{C}(10)), 130.2(\mathrm{C}(8)), 128.7(\mathrm{C}(2))$, 128.5 (C19)), $127.9(\mathrm{C}(3,24$, or 25$)), 126.9$ (C(12)), 126.2 (C(24 or 25$)), 125.9$ (C(24 or 25$)), 125.6(\mathrm{C}(21)), 125.4(\mathrm{C}(9)), 124.5(\mathrm{C}(3,24$, or 25$)), 85.1(\mathrm{C}(14))$, 73.5 (C(16)), $72.8(\mathrm{C}(5)), 71.0(\mathrm{C}(15)), 22.0(\mathrm{C}(13))$. 
IR: (thin-film)

$3057(\mathrm{w}), 2859(\mathrm{w}), 1641(\mathrm{~s}), 1602(\mathrm{w}), 1495(\mathrm{w}), 1454(\mathrm{~m}), 1365(\mathrm{w}), 1303(\mathrm{w})$,

$1275(\mathrm{~m}), 1124(\mathrm{~m}), 1077$ (m), 1046 (s), $891(\mathrm{w}), 818(\mathrm{~m}), 739(\mathrm{~m}), 6986(\mathrm{~m})$, $478(\mathrm{~m})$.

HRMS: $\left(\mathrm{ESI}^{+}\right)$Calcd for $\mathrm{C}_{28} \mathrm{H}_{26} \mathrm{NO}_{2}^{+}$: 408.1964, found: 408.1962.

Analysis: $\quad \mathrm{C}_{28} \mathrm{H}_{25} \mathrm{NO}_{2}$

Calcd: C, $82.53 \% ; H, 6.18 \% ; \mathrm{N}, 3.44 \%$

Found: C, 82.38\%; H, 6.15\%; N, 3.72\%

TLC: $\quad R_{f}=0.69(80: 20$ hexanes/ethyl acetate $)\left[\mathrm{UV} / \mathrm{KMnO}_{4}\right]$

CSP-HPLC: $\quad(S, R)-\mathbf{4 p} t_{R}=9.4 \mathrm{~min}(6.0 \%),(R, S)-\mathbf{4 p} t_{R}=12.6 \mathrm{~min}(94.0 \%)$ (Supelco Astec, hexanes/i-PrOH, 80:20, 1.0mL/min; $\left.220 \mathrm{~nm}, 24^{\circ} \mathrm{C}\right)$.

Opt. Rot.: $[\alpha]_{\mathrm{D}}^{23} 25.2\left(c=1.00, \mathrm{CHCl}_{3}\right)$

Preparation of $(4 R, 5 S)-5-((B e n z y l o x y) m e t h y l)-2-(2-m e t h y l p h e n y l)-4-(n a p h t h a l e n-1-y l)-2-(2-$ methylphenyl)-4,5-dihydrooxazole (4q).

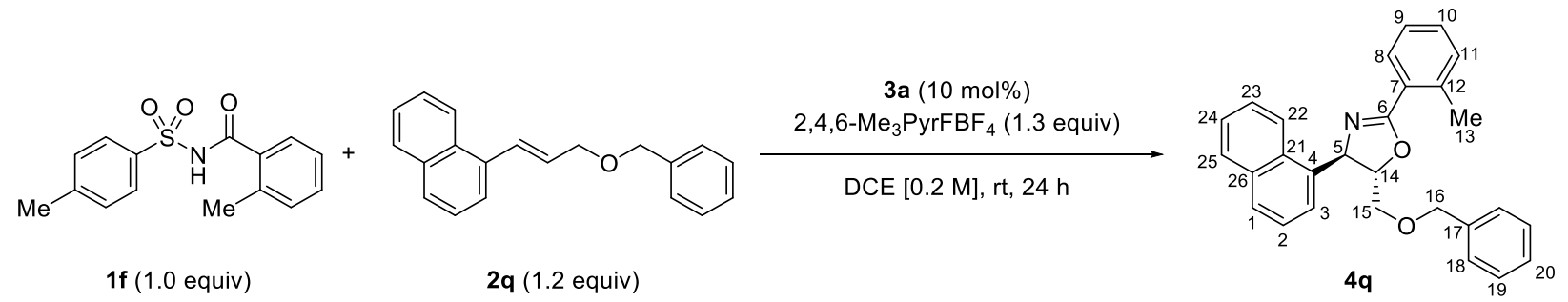

Following General Procedure I, 2-methyl- $N$-tosylbenzamide (289 mg, $1.00 \mathrm{mmol}$ ), diselenide catalyst 3a $(82.1 \mathrm{mg}, 0.10 \mathrm{mmol}, 0.10$ equiv $), 2,4,6-\mathrm{Me}_{3} \mathrm{PyF}^{+} \mathrm{BF}_{4}^{-}(295 \mathrm{mg}, 1.30 \mathrm{mmol}$, 1.30 equiv), and (E)-1-(3-(benzyloxy)prop-1-en-1-yl)naphthalene (329 mg, $1.20 \mathrm{mmol}, 1.20$ equiv) were combined in 1,2-DCE $(5.0 \mathrm{~mL}, 0.20 \mathrm{M})$ (alkene added as a solution in DCE) and allowed to stir for $24 \mathrm{~h}$ at $21{ }^{\circ} \mathrm{C}$. The resulting suspension was worked up as described in the 
General Procedure and purified by column chromatography (150 g silica gel, $5.5 \mathrm{~cm}$ column) eluting with a gradient from hexanes to hexanes/ethyl acetate, 89:11 (50-mL fractions) to yield $341 \mathrm{mg}(84 \%)$ of $\mathbf{4 q}$ as a clear, colorless oil. A $55.3 \mathrm{mg}$ portion was further purified by Kugelrohr distillation $\left(115-145{ }^{\circ} \mathrm{C}, 4.0 \times 10^{-5} \mathrm{~mm} \mathrm{Hg}\right)$ to afford $51.5 \mathrm{mg}$ of a clear colorless oil $(93 \%$ recovery).

Data for 4q:

b.p. $\quad 115-145^{\circ} \mathrm{C}\left(4.0 \times 10^{-5} \mathrm{~mm} \mathrm{Hg}\right)$

1H NMR: $\quad\left(500 \mathrm{MHz}, \mathrm{CDCl}_{3}\right)$

$\delta 8.09(\mathrm{~d}, J=8.5 \mathrm{~Hz}, 1 \mathrm{H}, \mathrm{HC}(22)), 7.99(\mathrm{~d}, J=7.7 \mathrm{~Hz}, 1 \mathrm{H}, \mathrm{HC}(8)), 7.88(\mathrm{~d}, J=$ $8.2 \mathrm{~Hz}, 1 \mathrm{H}, \mathrm{HC}(25)), 7.79$ (dd, $J=7.6,1.9 \mathrm{~Hz}, 1 \mathrm{H}, \mathrm{HC}(1)), 7.50-7.43$ (m, $3 \mathrm{H}$, $\mathrm{HC}(3,20,24)), 7.43-7.35(\mathrm{~m}, 6 \mathrm{H}, \mathrm{HC}(2,9,10,19,23)), 7.35-7.26(\mathrm{~m}, 3 \mathrm{H}$, $\mathrm{HC}(11,18)), 6.06(\mathrm{~d}, J=6.2 \mathrm{~Hz}, \mathrm{HC}(5)), 4.73\left(\mathrm{~d}, J=12.0 \mathrm{~Hz}, 1 \mathrm{H}, \mathrm{H}_{2} \mathrm{C}(16)\right)$, 4.69-4.63 (m, $\left.2 \mathrm{H}, \mathrm{HC}(14), \mathrm{H}_{2} \mathrm{C}(16)\right), 3.92$ (dd, $J=10.6,4.4 \mathrm{~Hz}, 1 \mathrm{H}, \mathrm{H}_{2} \mathrm{C}(15)$ ), $3.88\left(\mathrm{dd}, J=10.6,4.8 \mathrm{~Hz}, 1 \mathrm{H}, \mathrm{H}_{2} \mathrm{C}(15)\right), 2.72\left(\mathrm{~s}, 3 \mathrm{H}, \mathrm{H}_{3} \mathrm{C}(13)\right)$.

${ }^{13} \mathrm{C}$ NMR: $\quad\left(126 \mathrm{MHz}, \mathrm{CDCl}_{3}\right)$

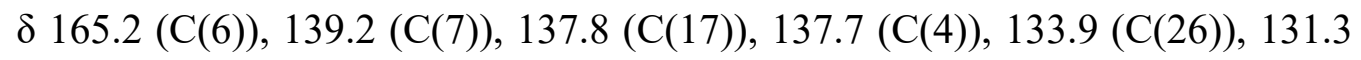
(C(11)), 130.8 (C(10)), $130.8(\mathrm{C}(21)), 130.2(\mathrm{C}(8)), 128.9$ (C(25)), 128.5 (C(19)), $128.1(\mathrm{C}(1)), 127.9(\mathrm{C}(2,18)), 126.9(\mathrm{C}(12)), 126.1(\mathrm{C}(23)), 125.7$ (C(20 or 24$)), 125.6(\mathrm{C}(20$ or 24$)), 125.6(\mathrm{C}(9)), 124.2(\mathrm{C}(3)), 123.1$ (C(22)), 84.6 (C(14)), $73.6(\mathrm{C}(16)), 70.6(\mathrm{C}(15)), 68.5(\mathrm{C}(5)), 22.1(\mathrm{C}(13))$.

IR: (thin-film) $2860(w), 1642(\mathrm{~s}), 1597(\mathrm{w}), 1511(\mathrm{w}), 1495(\mathrm{w}), 1454(\mathrm{~m}), 1360(\mathrm{w}), 1323(\mathrm{w})$, $1121(\mathrm{~m}), 1044$ (s), $889(\mathrm{w}), 799(\mathrm{~m}), 776(\mathrm{~s}), 729$ (s), $698(\mathrm{~m})$. 
HRMS: $\quad\left(\mathrm{ESI}^{+}\right)$Calcd for $\mathrm{C}_{28} \mathrm{H}_{26} \mathrm{NO}_{2}^{+}: 408.1964$, found: 408.1955 .

Analysis: $\quad \mathrm{C}_{28} \mathrm{H}_{25} \mathrm{NO}_{2}$

Calcd: C, 82.53\%; H, 6.18\%; N, 3.44\%

Found: C, 82.36\%; H, 6.18\%; N, 3.71\%

TLC: $\quad R_{f}=0.70(80: 20$ hexanes/ethyl acetate $)\left[\mathrm{UV} / \mathrm{KMnO}_{4}\right]$

CSP-HPLC: $\quad(R, S)-\mathbf{4 q} t_{R}=9.5 \min (88.0 \%),(S, R)-\mathbf{4 q} t_{R}=26.4 \min (12.0 \%)$ (Supelco Astec, hexanes/i-PrOH, 80:20, $\left.1.0 \mathrm{~mL} / \mathrm{min} ; 220 \mathrm{~nm}, 24{ }^{\circ} \mathrm{C}\right)$.

Opt. Rot.: $[\alpha]_{\mathrm{D}}^{23}-6.0\left(c=1.00, \mathrm{CHCl}_{3}\right)$

Preparation of (4R,5R)-2-(2-Methylphenyl)-4,5-diphenyl-4,5-dihydrooxazole (4r).

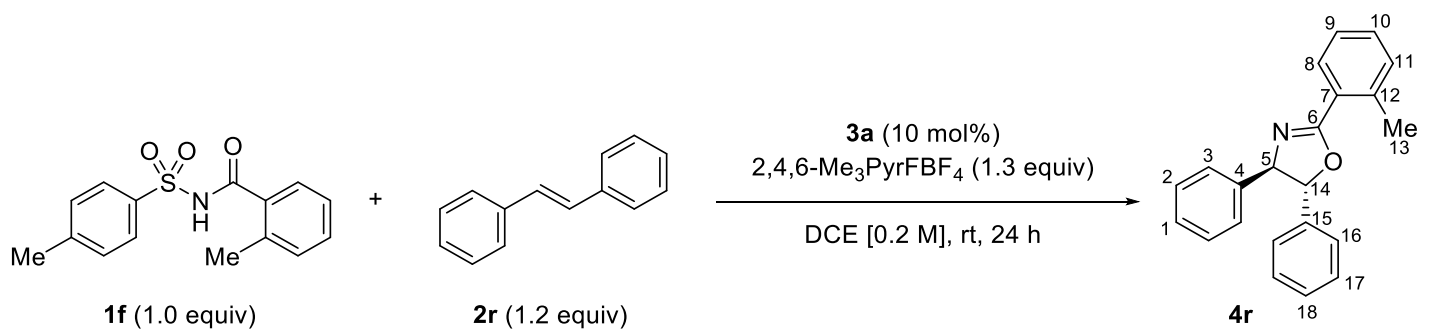

Following General Procedure I, 2-methyl- $N$-tosylbenzamide (289 mg, $1.00 \mathrm{mmol}$ ), diselenide catalyst $\mathbf{3 a}\left(82.1 \mathrm{mg}, 0.10 \mathrm{mmol}, 0.10\right.$ equiv), 2,4,6- $\mathrm{Me}_{3} \mathrm{PyF}^{+} \mathrm{BF}_{4}^{-}(295 \mathrm{mg}, 1.30 \mathrm{mmol}$, 1.30 equiv), and trans-stilbene (216 mg, $1.20 \mathrm{mmol}, 1.20$ equiv) were combined in 1,2-DCE (5.0 $\mathrm{mL}, 0.20 \mathrm{M}$ ) (alkene added neat) and allowed to stir for $24 \mathrm{~h}$ at $21^{\circ} \mathrm{C}$. The resulting suspension was worked up as described in the General Procedure and purified by column chromatography (150 g silica gel, $5.5 \mathrm{~cm}$ column) eluting with a gradient from hexanes to hexanes/ethyl acetate, 98:2 (20-mL fractions) to yield $245 \mathrm{mg}(78 \%)$ of $4 \mathbf{r}$ as a clear oil. A $100.3 \mathrm{mg}$ portion was further purified by Kugelrohr distillation $\left(115^{\circ} \mathrm{C}, 4.0 \times 10^{-5} \mathrm{~mm} \mathrm{Hg}\right)$ to afford $98.2 \mathrm{mg}$ of a clear oil (98\% recovery). 


\section{Data for 4r:}

b.p. $115^{\circ} \mathrm{C}\left(4.0 \times 10^{-5} \mathrm{~mm} \mathrm{Hg}\right)$

m.p. $\quad 48-50^{\circ} \mathrm{C}$ (distilled)

근 NMR: $\quad\left(500 \mathrm{MHz}, \mathrm{CDCl}_{3}\right)$

$\delta 8.01(\mathrm{dd}, J=7.8,1.4 \mathrm{~Hz}, 1 \mathrm{H}, \mathrm{HC}(8)), 7.44-7.35(\mathrm{~m}, 8 \mathrm{H}, \mathrm{HC}(2,3,10,16,18))$, 7.34-7.27 (m, $5 \mathrm{H}, \mathrm{HC}(1,9,11,17)), 5.35$ (d, J=7.6 Hz, $1 \mathrm{H}, \mathrm{HC}(14)), 5.28$ (d, $J=7.6 \mathrm{~Hz}, 1 \mathrm{H}, \mathrm{HC}(5)), 2.73\left(\mathrm{~s}, 3 \mathrm{H}, \mathrm{H}_{3} \mathrm{C}(13)\right)$.

${ }^{13} \mathrm{C}$ NMR: $\quad\left(126 \mathrm{MHz}, \mathrm{CDCl}_{3}\right)$

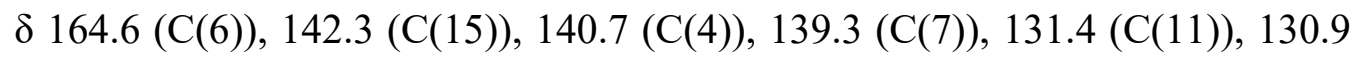
(C(8)), $130.2(\mathrm{C}(10)), 128.9(\mathrm{C}(17)), 128.8(\mathrm{C}(2)), 128.4(\mathrm{C}(18)), 127.7(\mathrm{C}(1))$, $126.8(\mathrm{C}(12)), 126.6(\mathrm{C}(3)), 125.7(\mathrm{C}(16)), 125.7(\mathrm{C}(9)), 88.2$ (C(14)), 79.4 $(\mathrm{C}(5)), 22.2(\mathrm{C}(13))$.

$\underline{\text { IR: (neat) }}$

3062 (w), 3028 (w), 2925 (w), $1941($ w), 1878 (w), $1805(w), 1723($ w), 1644 (m), 1602 (w), $1582(w), 1494(m), 1449$ (m), 1381 (w), 1338 (w), $1319(w)$, 1305 (w), 1269 (m), 1223 (w), 1198 (w), 1175 (w), 1159 (w), 1134 (w), 1123 (w), $1086(w), 1072$ (w), 1056 (m), 1043 (m), $1016(w), 1002(w), 961$ (m), 913 (w), $893(w), 870(w), 846(w), 780(w), 759(s), 731(s), 694(s), 672(m), 644$ (w), $618(\mathrm{w}), 604(\mathrm{~m}), 556(\mathrm{w}), 542(\mathrm{~m}), 511(\mathrm{w}), 466(\mathrm{~m})$

HRMS: $\quad\left(\mathrm{ESI}^{+}\right)$Calcd for $\mathrm{C}_{22} \mathrm{H}_{20} \mathrm{NO}^{+}: 314.1545$, found: 314.1536 .

Analysis: $\quad \mathrm{C}_{22} \mathrm{H}_{19} \mathrm{NO}$

Calcd: C, $84.31 \%$; H, $6.11 \% ; \mathrm{N}, 4.47 \%$

Found: $\mathrm{C}, 84.00 \%$; $\mathrm{H}, 6.06 \% ; \mathrm{N}, 4.66 \%$ 
TLC: $\quad R_{f}=0.43(16: 1$, hexanes/ethyl acetate) [UV]

CSP-HPLC: $\quad(S, S)-4 \mathbf{r} t_{R}=4.8 \mathrm{~min}(2.5 \%),(R, R)-4 \mathrm{r} t_{R}=5.5 \mathrm{~min}(97.5 \%)$ (Supelco Astec, hexanes/i-PrOH, 95:5, 1.0mL/min; $\left.220 \mathrm{~nm}, 24{ }^{\circ} \mathrm{C}\right)$.

Opt. Rot.: $[\alpha]_{\mathrm{D}}^{23}-21.49\left(c=1.06, \mathrm{CHCl}_{3}\right)$

\section{Preparation of $(4 R, 5 R)-4,5$-Bis(3-methoxyphenyl)-2-(2-methylphenyl)-4,5-dihydrooxazole}

(4s).

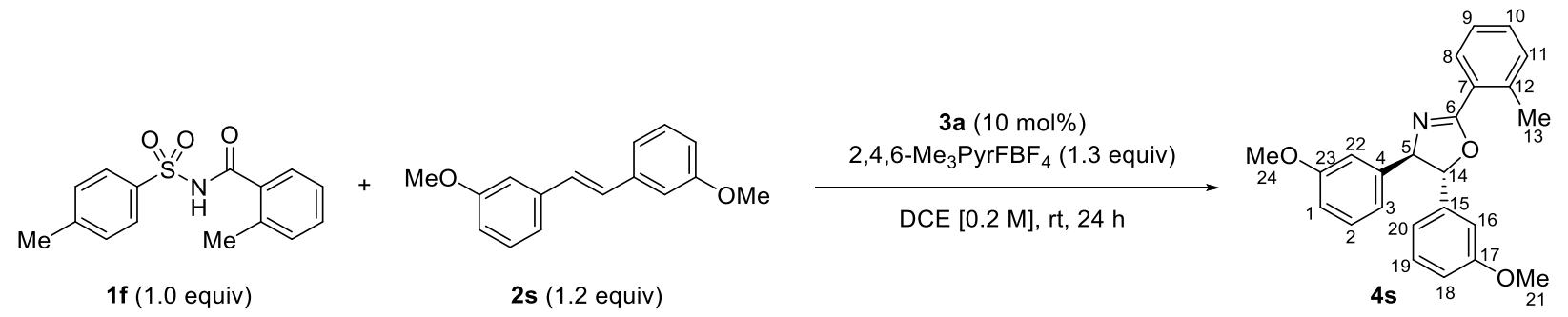

Following General Procedure I, 2-methyl- $N$-tosylbenzamide (289 mg, $1.00 \mathrm{mmol}$ ), diselenide catalyst 3a $\left(82.1 \mathrm{mg}, 0.10 \mathrm{mmol}, 0.10\right.$ equiv), 2,4,6- $\mathrm{Me}_{3} \mathrm{PyF}^{+} \mathrm{BF}_{4}^{-}$(295 mg, $1.30 \mathrm{mmol}$, 1.30 equiv), and (E)-1,2-bis(3-methoxyphenyl)ethene (288 mg, $1.20 \mathrm{mmol}, 1.20$ equiv) were combined in 1,2-DCE (5.0 mL, 0.20 M) (alkene added neat) and allowed to stir for $24 \mathrm{~h}$ at $21{ }^{\circ} \mathrm{C}$. The resulting suspension was worked up as described in the General Procedure and purified by column chromatography (150 g silica gel, $5.5 \mathrm{~cm}$ column) eluting with a hexanes/ethyl acetate gradient, $98: 2$ to $93: 7$ (20-mL fractions) to yield $229 \mathrm{mg}(61 \%)$ of $4 \mathrm{~s}$ as a clear oil. A $100.3 \mathrm{mg}$ portion was further purified by Kugelrohr distillation $\left(134^{\circ} \mathrm{C}, 3.4 \times 10^{-5} \mathrm{~mm} \mathrm{Hg}\right)$ to afford 93.4 mg of a clear oil ( $93 \%$ recovery).

Data for 4s:

b.p. $134{ }^{\circ} \mathrm{C}\left(3.4 \times 10^{-5} \mathrm{~mm} \mathrm{Hg}\right)$ 
1H NMR: $\quad\left(500 \mathrm{MHz}, \mathrm{CDCl}_{3}\right)$

$\delta 8.00(\mathrm{dd}, J=7.8,1.4 \mathrm{~Hz}, 1 \mathrm{H}, \mathrm{HC}(8)), 7.40(\operatorname{td}, J=7.6,1.5 \mathrm{~Hz}, 1 \mathrm{H}, \mathrm{HC}(10))$, 7.35-7.26 (m, 4 H, HC(2, 9, 11, 19)), 6.96 (dt, $J=7.5 \mathrm{~Hz}, 1.2 \mathrm{~Hz}, 1 \mathrm{H}, \mathrm{HC}(20))$, 6.93-6.84 (m, 5 H, HC(1, 3, 16, 18, 22)), 5.31 (d, $J=7.6 \mathrm{~Hz}, 1 \mathrm{H}, \mathrm{HC}(14)), 5.25$ (d, $J=7.6 \mathrm{~Hz}, 1 \mathrm{H}, \mathrm{HC}(5)), 3.81$ (s, $3 \mathrm{H}, \mathrm{H}_{3} \mathrm{C}(21$ or 24$\left.)\right), 3.81$ (s, $3 \mathrm{H}, \mathrm{H}_{3} \mathrm{C}(21$ or 24)), 2.73 (s, $\left.3 \mathrm{H}, \mathrm{H}_{3} \mathrm{C}(13)\right)$.

${ }^{13} \mathrm{C} \mathrm{NMR}: \quad\left(126 \mathrm{MHz}, \mathrm{CDCl}_{3}\right)$

$\delta 164.6(\mathrm{C}(6)), 160.0(\mathrm{C}(17$ or 23$)), 160.0(\mathrm{C}(17$ or 23$)), 143.9(\mathrm{C}(15)), 142.3$ (C(4)), $139.3(\mathrm{C}(7)), 131.4(\mathrm{C}(11)), 130.9(\mathrm{C}(10)), 130.2(\mathrm{C}(8)), 130.0(\mathrm{C}(2$ or 19)), 129.9 (C(2 or 19)), $126.8(\mathrm{C}(12)), 125.7(\mathrm{C}(9)), 119.0(\mathrm{C}(3)), 118.0(\mathrm{C}(20))$, $113.7(\mathrm{C}(1$ or 18$)), 113.1(\mathrm{C}(1$ or 18$)), 112.2(\mathrm{C}(22)), 111.3(\mathrm{C}(16)), 87.9$ (C(14)), $79.2(\mathrm{C}(5)), 55.3(\mathrm{C}(21$ or 24$)), 55.3(\mathrm{C}(21$ or 24$)), 22.2(\mathrm{C}(13))$.

IR: (neat)

$3000(\mathrm{w}), 2935(\mathrm{w}), 2835(\mathrm{w}), 1931(\mathrm{w}), 1640(\mathrm{~m}), 1600(\mathrm{~m}), 1585(\mathrm{~m}), 1489$ (m), 1454 (m), 1435 (m), 1383 (w), 1309 (m), 1263 (s), 1192 (w), 1155 (m), 1137 (w), 1075 (w), 1042 (s), 995 (m), 977 (m), 897 (m), 870 (m), 768 (s), 729 (s), $695(\mathrm{~s}), 673(\mathrm{~m}), 554(\mathrm{w}), 457(\mathrm{w})$

HRMS: (ESI ${ }^{+}$) Calcd for $\mathrm{C}_{24} \mathrm{H}_{24} \mathrm{NO}_{3}{ }^{+}$: 374.1756 , found: 374.1741 .

Analysis: $\mathrm{C}_{24} \mathrm{H}_{23} \mathrm{NO}_{3}$

Calcd: C, 77.19\%; H, 6.21\%; N, 3.75\%

Found: C, $77.28 \% ; \mathrm{H}, 6.23 \% ; \mathrm{N}, 4.01 \%$

TLC: $R_{f}=0.16(16: 1$ hexanes/ethyl acetate $)[\mathrm{UV}]$ 
CSP-HPLC: $\quad(R, R)-4 \mathrm{~s} t_{R}=7.93 \min (94.8 \%),(S, S)-4 \mathrm{~s} t_{R}=9.97 \mathrm{~min}(5.2 \%)($ Regis $R, R$-WhelkO1, hexanes/i-PrOH, 70:30, $\left.1.0 \mathrm{~mL} / \mathrm{min} .220 \mathrm{~nm}, 24{ }^{\circ} \mathrm{C}\right)$.

Opt. Rot.: $[\alpha]_{\mathrm{D}}^{23}-12.9\left(c=0.44, \mathrm{CHCl}_{3}\right)$

Preparation of

(4R,5R)-4-(4-Methoxyphenyl)-2-(2-methylphenyl)-5-(4-

(trifluoromethyl)phenyl)-4,5-dihydrooxazole (4t).

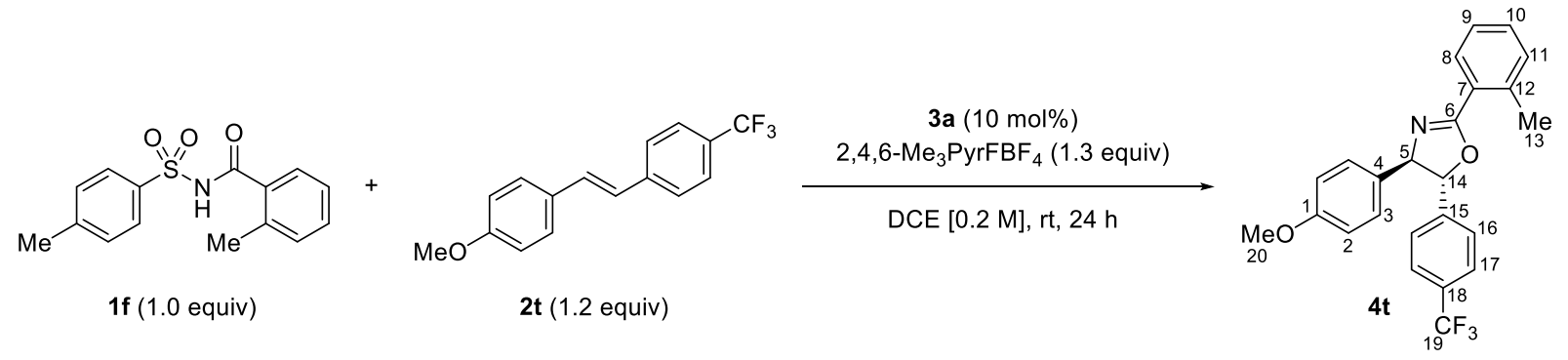

Following General Procedure I, 2-methyl- $N$-tosylbenzamide (289 mg, $1.00 \mathrm{mmol}$ ), diselenide catalyst 3a $\left(82.1 \mathrm{mg}, 0.10 \mathrm{mmol}, 0.10\right.$ equiv), 2,4,6- $\mathrm{Me}_{3} \mathrm{PyF}^{+} \mathrm{BF}_{4}^{-}(295 \mathrm{mg}, 1.30 \mathrm{mmol}$, 1.30 equiv), and (E)-1-methoxy-4-(4-trifluoromethyl)styryl)benzene (334 mg, $1.20 \mathrm{mmol}, 1.20$ equiv) were combined in 1,2-DCE (5.0 mL, 0.20 M) (alkene added as a solid) and allowed to stir for $24 \mathrm{~h}$ at $21^{\circ} \mathrm{C}$. The resulting suspension was worked up as described in the General Procedure and purified by column chromatography (150 g silica gel, $5.5 \mathrm{~cm}$ column) eluting with a hexanes/ethyl acetate gradient, $97: 3$ to $93: 7$ (20-mL fractions) to yield $360 \mathrm{mg}(88 \%)$ of $\mathbf{4 t}$ as a pale yellow solid. Recrystallization from ca. $1 \mathrm{~mL}$ boiling hexanes (cooled in freezer overnight) afforded $324 \mathrm{mg}$ of a yellow-white solid (90\% recovery).

Data for 4t:

m.p. $\quad 74{ }^{\circ} \mathrm{C}$ (hexane) 
${ }^{1} \mathrm{H}$ NMR: $\quad\left(500 \mathrm{MHz}, \mathrm{CDCl}_{3}\right)$

$\delta 8.01(\mathrm{dd}, J=7.8,1.4 \mathrm{~Hz}, 1 \mathrm{H}, \mathrm{HC}(8)), 7.67(\mathrm{~d}, J=8.1 \mathrm{~Hz}, 2 \mathrm{H}, \mathrm{HC}(17)), 7.47$

$(\mathrm{d}, J=8.1 \mathrm{~Hz}, 2 \mathrm{H}, \mathrm{HC}(16)), 7.42(\mathrm{td}, J=7.5,1.5 \mathrm{~Hz}, 1 \mathrm{H}, \mathrm{HC}(10)), 7.34-7.27$

(m, $2 \mathrm{H}, \mathrm{HC}(9,11)), 7.23(\mathrm{~d}, J=8.7 \mathrm{~Hz}, 2 \mathrm{H}, \mathrm{HC}(3)), 6.93(\mathrm{~d}, J=8.7 \mathrm{~Hz}, 2 \mathrm{H}$,

HC(2)), $5.36(\mathrm{~d}, J=7.8 \mathrm{~Hz}, 1 \mathrm{H}, \mathrm{HC}(14)), 5.17$ (d, $J=7.8 \mathrm{~Hz}, 1 \mathrm{H}, \mathrm{HC}(5)), 3.83$

(s, $\left.3 \mathrm{H}, \mathrm{H}_{3} \mathrm{C}(20)\right), 2.72$ (s, $\left.3 \mathrm{H}, \mathrm{H}_{3} \mathrm{C}(13)\right)$.

${ }^{13} \mathrm{C} \mathrm{NMR:} \quad\left(126 \mathrm{MHz}, \mathrm{CDCl}_{3}\right)$

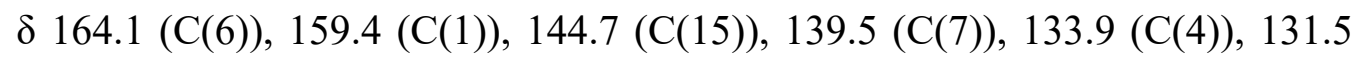

(C(11)), $131.1(\mathrm{C}(10)), 130.5\left(\mathrm{q},{ }^{2} J_{C-F}=32.6 \mathrm{~Hz}, \mathrm{C}(18)\right), 130.2(\mathrm{C}(8)), 127.8$

(C(3)), $126.4(\mathrm{C}(12)), 125.9\left(\mathrm{q},{ }^{3} J_{C-F}=3.9 \mathrm{~Hz}, \mathrm{C}(17)\right), 125.8(\mathrm{C}(16)), 125.8$

(C(9)), $124.0\left(\mathrm{q},{ }^{1} J_{C-F}=272.3 \mathrm{~Hz}, \mathrm{C}(19)\right), 114.3(\mathrm{C}(2)), 87.4(\mathrm{C}(14)), 79.2$

$(\mathrm{C}(5)), 55.3(\mathrm{C}(20)), 22.3(\mathrm{C}(13))$.

${ }^{19} \mathrm{~F}$ NMR: $\quad\left(471 \mathrm{MHz}, \mathrm{CDCl}_{3}\right)$

$\delta-62.5$

IR: (neat)

$3009(w), 2960(w), 2913(w), 2858(w), 2838(w), 2051(w), 1931(w), 1641$

(m), $1619(\mathrm{~m}), 1586(\mathrm{w}), 1514(\mathrm{~m}), 1495(\mathrm{w}), 1468(\mathrm{w}), 1455(\mathrm{~m}), 1421(\mathrm{w})$, 1384 (w), 1321 (s), 1305 (m), 1269 (m), 1246 (s), 1199 (w), 1176 (m), 1161 (s), 1124 (s), 1108 (s), 1067 (s), 1047 (s), 1034 (s), 1015 (m), 984 (s), 956 (w), 939 (w), $900(w), 893(w), 866(w), 842(s), 836($ s), $828(s), 814(\mathrm{~m}), 777(\mathrm{~m}), 767$ (m), $731(\mathrm{~s}), 697(\mathrm{w}), 682(\mathrm{~m}), 663(\mathrm{~m}), 650(\mathrm{w}), 626(\mathrm{w}), 605(\mathrm{~m}), 589(\mathrm{~m}), 546$ $(\mathrm{s}), 524(\mathrm{w}), 485(\mathrm{~m})$

HRMS: $\quad\left(\mathrm{ESI}^{+}\right)$Calcd for $\mathrm{C}_{24} \mathrm{H}_{21} \mathrm{~F}_{3} \mathrm{NO}_{2}{ }^{+}:$412.1524, found: 412.1510 


\section{Analysis: $\mathrm{C}_{24} \mathrm{H}_{20} \mathrm{~F}_{3} \mathrm{NO}_{2}$}

Calcd: C, 70.06\%; H, 4.90\%; N, 3.40\%

Found: C, 70.27\%; H, 5.02\%; N, 3.52\%

TLC: $\quad R_{f}=0.18$ (94:6 hexanes/ethyl acetate) [UV]

CSP-HPLC: $\quad(R, R)-\mathbf{4 t} t_{R}=5.81 \mathrm{~min}(97.7 \%),(S, S)-\mathbf{4 t} t_{R}=8.25 \mathrm{~min}(2.28 \%)($ Regis $R, R-$ Whelk-O1, hexanes/i-PrOH, 95:5, $1.0 \mathrm{~mL} / \mathrm{min} .220 \mathrm{~nm}, 24^{\circ} \mathrm{C}$ ).

Opt. Rot.: $[\alpha]_{\mathrm{D}}{ }^{23}-4.6\left(c=1.18, \mathrm{CHCl}_{3}\right)$

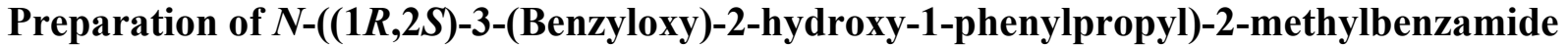

(5).

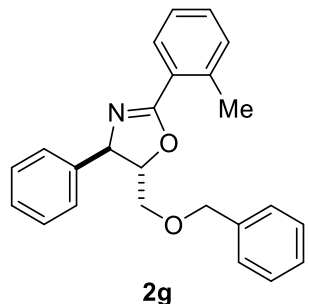

$2 \mathrm{~g}$
$\mathrm{HCl}$ (aq, $1.0 \mathrm{~N}, 5.1$ equiv), MeOH/THF, reflux, $19 \mathrm{~h}$

then, $\mathrm{NaHCO}_{3}$, rt, $3.5 \mathrm{~h}$

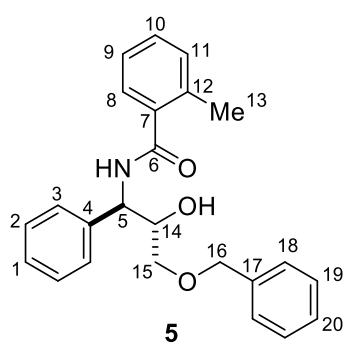

$1.0 \mathrm{mmol}$ reaction on racemic starting material for characterization

To a solution of \pm -trans-5-(benzyloxy)methyl)-4-phenyl-2-(o-tolyl)-4,5-dihydrooxazole (367 mg, $1.0 \mathrm{mmol}, 1.0$ equiv) in methanol $(10.5 \mathrm{~mL})$ and tetrahydrofuran $(10.5 \mathrm{~mL})$ was added hydrochloric acid $(1.0 \mathrm{~N}, 5.1 \mathrm{~mL}, 5.1 \mathrm{mmol}, 5.1$ equiv) dropwise at room temperature. The resulting solution was stirred at reflux for $19 \mathrm{~h}$. After cooling to room temperature, a saturated aqueous solution of sodium bicarbonate $(24 \mathrm{~mL})$ was added and the slurry was stirred at room temperature for $3.5 \mathrm{~h}$. After decanting, the aqueous layer was extracted with ethyl acetate $(4 \times 75$ $\mathrm{mL}$ ). The combined organic layers were dried with magnesium sulfate and concentrated under reduced pressure by rotary evaporation $\left(30^{\circ} \mathrm{C}, \sim 20 \mathrm{~mm} \mathrm{Hg}\right)$ to yield $894 \mathrm{mg}$ of a cloudy oil. 
Column chromatography on Teledyne ISCO Combi-Flash (silica gel, 120 g Luknova SuperSep column) eluting with a gradient from hexanes to hexanes/ethyl acetate, 50:50 to yield $354 \mathrm{mg}$ (94\%) of $4 \mathbf{a}$ as a white solid. Recrystallization from ca. $5 \mathrm{~mL}$ of boiling methanol (cooled in freezer overnight) afforded $264 \mathrm{mg}$ of a white solid (70\%).

0.2 mmol reaction on enantioenriched starting material for CSP-HPLC/optical rotation

To a solution of (4R,5R)-5-(benzyloxy)methyl)-4-phenyl-2-(o-tolyl)-4,5-dihydrooxazole (72 $\mathrm{mg}, 0.2 \mathrm{mmol}, 1.0$ equiv) in methanol $(2.0 \mathrm{~mL})$ and tetrahydrofuran $(2.0 \mathrm{~mL})$ was added hydrochloric acid $(1.0 \mathrm{~N}, 1.0 \mathrm{~mL}, 1.0 \mathrm{mmol}, 5.0$ equiv) dropwise at room temperature. The resulting solution was stirred at reflux for $18 \mathrm{~h}$. After cooling to room temperature, a saturated aqueous solution of sodium bicarbonate $(4.8 \mathrm{~mL})$ was added and the slurry was stirred at room temperature for $5 \mathrm{~h}$. After decanting, the aqueous layer was extracted with ethyl acetate $(4 \times 15$ $\mathrm{mL}$ ). The combined organic layers were dried with magnesium sulfate and concentrated under reduced pressure by rotary evaporation $\left(30^{\circ} \mathrm{C}, \sim 20 \mathrm{~mm} \mathrm{Hg}\right)$ to yield $75 \mathrm{mg}$ of a cloudy oil. Column chromatography on Teledyne ISCO Combi-Flash (silica gel, 25 g Luknova SuperSep column) eluting with a gradient from hexanes to hexanes/ethyl acetate, 50:50 to afford $55 \mathrm{mg}$ of a white solid $(73 \%)$.

Data for 5:

1H NMR: $\quad\left(500 \mathrm{MHz}, \mathrm{CDCl}_{3}\right)$

$\delta 7.42-7.27(\mathrm{~m}, 12 \mathrm{H}, \mathrm{HC}(1-3,8,10,18-20)), 7.22(\mathrm{~d}, J=7.4 \mathrm{~Hz}, 1 \mathrm{H}, \mathrm{HC}(11))$, 7.19 (t, $J=7.6 \mathrm{~Hz}, 1 \mathrm{H}, \mathrm{HC}(9)), 6.71(\mathrm{~d}, J=8.3 \mathrm{~Hz}, 1 \mathrm{H}, \mathrm{HN}), 5.27$ (dd, $J=8.3$, $3.6 \mathrm{~Hz}, 1 \mathrm{H}, \mathrm{HC}(5)), 4.59$ (d, $\left.J=11.8 \mathrm{~Hz}, 1 \mathrm{H}, \mathrm{H}_{2} \mathrm{C}(16)\right), 4.54$ (d, $J=11.8 \mathrm{~Hz}, 1$ $\left.\mathrm{H}, \mathrm{H}_{2} \mathrm{C}(16)\right), 4.20$ (dq, $\left.J=7.7,3.9 \mathrm{~Hz}, 1 \mathrm{H}, \mathrm{HC}(14)\right), 3.64$ (dd, $J=9.6,4.2 \mathrm{~Hz}, 1$ $\left.\left.\mathrm{H}, \mathrm{H}_{2} \mathrm{C}(15)\right), 3.52 \mathrm{dd}, J=9.6,7.3 \mathrm{~Hz}, 1 \mathrm{H}, \mathrm{H}_{2} \mathrm{C}(15)\right), 2.61(\mathrm{~d}, J=3.9 \mathrm{~Hz}, 1 \mathrm{H}$, 
HO), 2.40 (s, $\left.3 \mathrm{H}, \mathrm{H}_{3} \mathrm{C}(13)\right)$.

${ }^{13} \mathrm{C} \mathrm{NMR:} \quad\left(126 \mathrm{MHz}, \mathrm{CDCl}_{3}\right)$

$\delta 169.7(\mathrm{C}(6)), 140.0(\mathrm{C}(4)), 137.5(\mathrm{C}(17)), 136.3(\mathrm{C}(7$ or 12$)), 136.1(\mathrm{C}(7$ or 12)), $131.1(\mathrm{C}(11)), 130.0(\mathrm{C}(10)), 128.7$ (C(2 or 19)), 128.5 (C(2 or 19)), 128.0 (C(1 or 20$)), 127.9(\mathrm{C}(18)), 127.6(\mathrm{C}(1$ or 20$)), 126.9(\mathrm{C}(3)), 126.8(\mathrm{C}(8)), 125.7$ (C(9)), $73.6(\mathrm{C}(16)), 73.1(\mathrm{C}(14)), 71.6(\mathrm{C}(15)), 54.6(\mathrm{C}(5)), 19.9(\mathrm{C}(13))$.

IR: (neat) $3309(\mathrm{w}), 3062$ (w), $2910(\mathrm{w}), 2859(\mathrm{w}), 1635$ (s), $1600(\mathrm{w}), 1579(\mathrm{w}), 1529(\mathrm{~s})$, 1493 (m), 1454 (m), 1355 (m), 1320 (m), 1290 (w), 1242 (w), 1204 (w), 1161 (w), $1104(\mathrm{~m}), 1067(\mathrm{~m}), 1029$ (w), $1012(\mathrm{~m}), 934(\mathrm{w}), 911(\mathrm{w}), 895(\mathrm{~m}), 867$ (m), $834(w), 797(w), 781(w), 757(m), 741(m), 722(m), 696(s), 619(m)$

HRMS: $\quad\left(\mathrm{ESI}^{+}\right)$Calcd for $\mathrm{C}_{24} \mathrm{H}_{26} \mathrm{NO}_{3}+:$ 376.1913, found: 376.1906 .

Analysis: $\mathrm{C}_{24} \mathrm{H}_{25} \mathrm{NO}_{3}$

Calcd: C, 76.77\%; H, 6.71\%; N, 3.73\%

Found: C, 76.99\%; H, 6.69\%; N, 3.65\%

TLC: $\quad R_{f}=0.54$ (50:50 hexanes/ethyl acetate) [UV]

CSP-HPLC: $\quad(R, S)-5 t_{R}=7.97 \min (94.6 \%),(S, R)-5 t_{R}=11.53 \min (5.4 \%)($ Regis $R, R$-WhelkO1, hexanes/i-PrOH, 70:30, 1.0mL/min; $\left.220 \mathrm{~nm}, 24^{\circ} \mathrm{C}\right)$.

Opt. Rot.: $[\alpha]_{\mathrm{D}}{ }^{23}-4.6\left(c=0.96, \mathrm{CHCl}_{3}\right)$ 


\section{Preparation of $(1 R, 2 S)-1-A$ mino-3-(benzyloxy)-1-phenylpropan-2-yl 2-methylbenzoate}

\section{Hydrochloride (6).}

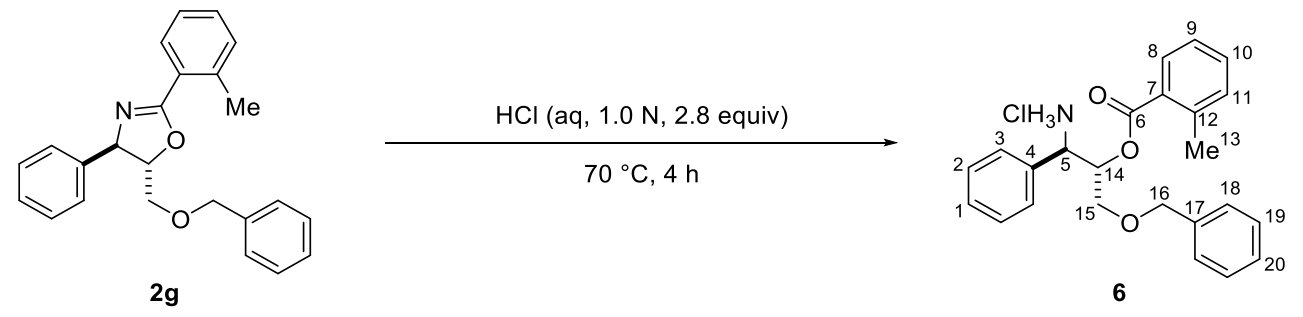

$\underline{1.0 \mathrm{mmol} \text { reaction on racemic starting material for characterization }}$

To neat 土-trans-5-(benzyloxy)methyl)-4-phenyl-2-(o-tolyl)-4,5-dihydrooxazole (379 mg, $1.06 \mathrm{mmol}, 1.0$ equiv) was added hydrochloric acid $(1.0 \mathrm{~N}, 2.9 \mathrm{~mL}, 2.9 \mathrm{mmol}, 2.8$ equiv) at room temperature. The resulting solution was stirred at $70^{\circ} \mathrm{C}$ for $4 \mathrm{~h}$. After cooling to room temperature, the solution was diluted in methanol $(10 \mathrm{~mL})$ and concentrated under reduced pressure by rotary evaporation $\left(30^{\circ} \mathrm{C}, \sim 20 \mathrm{~mm} \mathrm{Hg}\right.$ ) to afford $420 \mathrm{mg}$ of a white solid. Recrystallization from ca. 3 $\mathrm{mL}$ of boiling tetrahydrofuran (cooled in freezer overnight) afforded $377 \mathrm{mg}$ of a white solid $(86 \%)$.

Small-scale reaction on enantioenriched starting material for CSP-HPLC/optical rotation

To neat (1R,2S)-5-(benzyloxy)methyl)-4-phenyl-2-(o-tolyl)-4,5-dihydrooxazole (57.2 mg, $0.16 \mathrm{mmol})$ was added hydrochloric acid (1.0 N, $445 \mu \mathrm{L}, 0.45 \mathrm{mmol}, 2.8$ equiv) at room temperature. The resulting solution was stirred at $70^{\circ} \mathrm{C}$ for $4 \mathrm{~h}$. After cooling to room temperature, the solution was diluted in methanol $(1 \mathrm{~mL})$ and concentrated under reduced pressure by rotary evaporation $\left(30^{\circ} \mathrm{C}, \sim 20 \mathrm{~mm} \mathrm{Hg}\right)$ to afford $70 \mathrm{mg}$ of a white solid. The solid was washed with diethyl ether $(3 \times 5 \mathrm{~mL})$ on a medium porosity fritted funnel and the diethyl ether was washed $3 \mathrm{x}$ with $10 \mathrm{~mL}$ of $1 \mathrm{~N} \mathrm{HCl}$. The aqueous layer was concentrated under reduced pressure by rotary evaporation $\left(30^{\circ} \mathrm{C}, \sim 20 \mathrm{~mm} \mathrm{Hg}\right)$ to afford $16.4 \mathrm{mg}$ of a white solid. The material collected by filtration was suspended in diethyl ether $(15 \mathrm{~mL})$ and washed with $\mathrm{HCl}(3 \mathrm{x} 10 \mathrm{~mL})$. The two 
layers were separately concentrated under reduced pressure by rotary evaporation $\left(30^{\circ} \mathrm{C}, \sim 20 \mathrm{~mm}\right.$ $\mathrm{Hg}$ ) to afford a total of $43.9 \mathrm{mg}$ of a white solid. The combined $60.3 \mathrm{mg}$ was triturated in hexanes $(1 \mathrm{~mL})$ to afford $43.8 \mathrm{mg}$ of a yellow solid (66\%).

Data for 6 :

m.p.: $130^{\circ} \mathrm{C}($ decomp $)$

1H NMR: $\quad\left(500 \mathrm{MHz}, \mathrm{MeOH}-\mathrm{d}_{4}\right)$

$\delta 8.06(\mathrm{dd}, J=8.1,1.5 \mathrm{~Hz}, 1 \mathrm{H}, \mathrm{HC}(8)), 7.53-7.47$ (m, $6 \mathrm{H}, \mathrm{HC}(1,3,19,20))$, 7.34 (dt, $J=7.6,3.7 \mathrm{~Hz}, 2 \mathrm{H}, \mathrm{HC}(9,10))$, 7.28-7.24 (m, $5 \mathrm{H}, \mathrm{HC}(2,11,18)$ ), $5.60(\mathrm{dt}, J=8.8,3.2 \mathrm{~Hz}, 1 \mathrm{H}, \mathrm{HC}(14)), 4.84$ (d, $J=8.9 \mathrm{~Hz}, 1 \mathrm{H}, \mathrm{HC}(5)), 4.47$ (d, $\left.J=11.9 \mathrm{~Hz}, 1 \mathrm{H}, \mathrm{H}_{2} \mathrm{C}(16)\right), 4.43\left(\mathrm{~d}, J=11.9 \mathrm{~Hz}, 1 \mathrm{H}, \mathrm{H}_{2} \mathrm{C}(16)\right), 3.69$ (dd, $J=$ 11.2, $\left.2.8 \mathrm{~Hz}, 1 \mathrm{H}, \mathrm{H}_{2} \mathrm{C}(15)\right), 3.43$ (dd, $\left.J=11.2,3.6 \mathrm{~Hz}, 1 \mathrm{H}, \mathrm{H}_{2} \mathrm{C}(15)\right), 2.58$ (s, 3 $\left.\mathrm{H}, \mathrm{H}_{3} \mathrm{C}(13)\right)$.

${ }^{13} \mathrm{C} \mathrm{NMR}: \quad\left(126 \mathrm{MHz}, \mathrm{MeOH}-\mathrm{d}_{4}\right)$

8167.8 (C(6)), $142.0(\mathrm{C}(4)), 138.7(\mathrm{C}(17)), 135.0(\mathrm{C}(7)), 133.9(\mathrm{C}(10)), 132.9$ (C(11)), $132.0(\mathrm{C}(8)), 131.1(\mathrm{C}(1)), 130.6(\mathrm{C}(2$ or 19)), $129.9(\mathrm{C}(12)), 129.5(\mathrm{C}(2$ or 19)), $129.1(\mathrm{C}(18)), 129.0(\mathrm{C}(3,20)), 127.0(\mathrm{C}(9)), 74.9(\mathrm{C}(14)), 74.4(\mathrm{C}(16))$, $68.9(\mathrm{C}(15)), 56.8(\mathrm{C}(5)), 21.8(\mathrm{C}(13))$.

IR: (neat)

2861 (m), 1981 (w), 1709 (s), 1604 (m), 1557 (w), 1496 (m), 1454 (m), 1439 (w), $1358(w), 1336(w), 1290(m), 1250(\mathrm{~s}), 1206(\mathrm{w}), 1183(\mathrm{w}), 1139(\mathrm{~m}), 1121$ (m), $1086(\mathrm{~s}), 1066(\mathrm{~s}), 1029(\mathrm{~m}), 968(\mathrm{w}), 918(\mathrm{w}), 888(\mathrm{w}), 875$ (w), $800(\mathrm{w})$, $781(\mathrm{w}), 767(\mathrm{~m}), 736(\mathrm{~s}), 699(\mathrm{~s}), 659(\mathrm{w})$

HRMS: (ESI ${ }^{+}$Calcd for $\mathrm{C}_{24} \mathrm{H}_{26} \mathrm{NO}_{3}{ }^{+}$: 376.1913 , found: 376.1907 . 


\section{Analysis: $\quad \mathrm{C}_{24} \mathrm{H}_{26} \mathrm{ClNO}_{3}$}

Calcd: C, 69.98\%; H, 6.36; N, 3.40\%

Found: C, 70.04\%; H, 6.38; N, 3.35\%

TLC: $\quad R_{f}=0.45(50: 50$ hexanes/ethyl acetate) $[\mathrm{UV}]$

Opt. Rot.: $[\alpha]_{\mathrm{D}}^{23}-18.7(c=0.87, \mathrm{MeOH})$

Preparation of (1R,2S)-1-Amino-3-(benzyloxy)-1-phenylpropan-2-ol Hydrochloride (7).

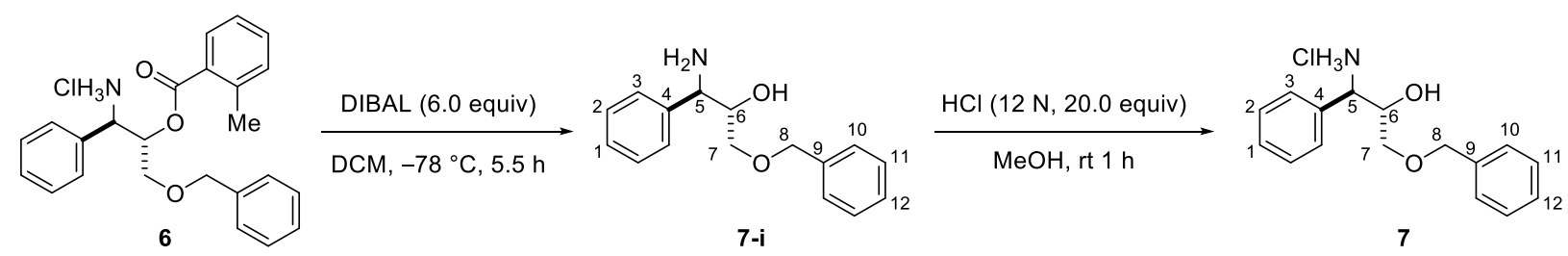

1.0 mmol reaction on racemic starting material for characterization

To a solution of \pm -trans-1-amino-3-(benzyloxy)-1-phenylpropan-2-yl 2-methylbenzoate hydrochloride (419 mg, $1.02 \mathrm{mmol}, 1.00$ equiv.) in dichloromethane $(36 \mathrm{~mL})$ at $-78^{\circ} \mathrm{C}$ was added a solution of diisobutylaluminum hydride (1.00 M in hexanes, $6.10 \mathrm{~mL}, 6.10 \mathrm{mmol}, 6.00$ equiv.). The resulting solution was stirred at $-78^{\circ} \mathrm{C}$ for $5.5 \mathrm{~h}$. The reaction was quenched with methanol $(1.5 \mathrm{~mL})$ at $-78^{\circ} \mathrm{C}$ and stirred overnight while warming to room temperature. The mixture was treated sequentially with $2.7 \mathrm{~mL}$ water, $2.7 \mathrm{~mL} 15 \% \mathrm{NaOH}$, and $8.1 \mathrm{~mL}$ water, then filtered through celite, washing with dichloromethane. The crude product was purified by column chromatography on Teledyne ISCO Combi-Flash (silica gel, $80 \mathrm{~g}$ Luknova SuperSep column) eluting with methanol/ ethyl acetate gradient from $0 \%$ to $50 \%$ methanol to afford $236.1 \mathrm{mg}$ of an off-white solid (90\%). The material was further purified by precipitation from minimal hexanes in THF (approx. 4:1 THF: hexanes; $1 \mathrm{~mL}$ total solvent volume) to afford $158.1 \mathrm{mg}$ of 7-i as an offwhite solid (67\% recovery). A $53.8 \mathrm{mg}$ portion was further purified by Kugelrohr distillation (95- 
$\left.100{ }^{\circ} \mathrm{C}, 5.0 \times 10^{-5} \mathrm{~mm} \mathrm{Hg}\right)$ to afford a white solid.

To a solution of t-trans-1-amino-3-(benzyloxy)-1-phenylpropan-2-ol (265 mg, 1.03 mmol) in methanol $(20.6 \mathrm{~mL})$ in a $50 \mathrm{~mL}$ Schlenk flask under a nitrogen atmosphere was added hydrochloric acid (1.5 mL, $12 \mathrm{~N}, 18.02 \mathrm{mmol}, 17.5$ equiv). The resulting solution was stirred for $1 \mathrm{~h}$ at room temperature, then diluted with methanol $(10 \mathrm{~mL})$ and concentrated under reduced pressure by rotary evaporation $\left(30^{\circ} \mathrm{C}, \sim 20 \mathrm{~mm} \mathrm{Hg}\right)$ to yield $297.7 \mathrm{mg}$ of a pale-yellow solid (98\%). The solid was triturated with refluxing diethyl ether $(2 \mathrm{~mL})$ and filtered to yield $267.9 \mathrm{mg}$ of 7 as a white solid.

$\underline{\text { Small-scale reaction on enantioenriched starting material for CSP-HPLC/optical rotation }}$

To a solution of $(1 R, 2 S)$-1-Amino-3-(benzyloxy)-1-phenylpropan-2-yl 2-methylbenzoate hydrochloride $\left(32.0 \mathrm{mg}, 0.077 \mathrm{mmol}, 1.00\right.$ equiv.) in dichloromethane $(2.8 \mathrm{~mL})$ at $-78^{\circ} \mathrm{C}$ was added a solution of diisobutylaluminum hydride (1.00 M in heptane, $0.466 \mathrm{~mL}, 0.466 \mathrm{mmol}, 6.00$ equiv.). The resulting solution was stirred at $-78^{\circ} \mathrm{C}$ for $5.5 \mathrm{~h}$. The reaction was quenched with methanol $(0.12 \mathrm{~mL})$ at $-78^{\circ} \mathrm{C}$ and stirred overnight while warming to room temperature. The mixture was treated sequentially with $0.21 \mathrm{~mL}$ water, $0.21 \mathrm{~mL} 15 \% \mathrm{NaOH}$, and $0.63 \mathrm{~mL}$ water, then filtered through celite, washing with dichloromethane. The crude product was purified by column chromatography on Teledyne ISCO Combi-Flash (silica gel, 4 g SiliCycle column) eluting with methanol/ ethyl acetate gradient from $0 \%$ to $50 \%$ methanol to afford $12.0 \mathrm{mg}$ of a white solid $(61 \%)$

To a solution of $(1 R, 2 S)$-1-amino-3-(benzyloxy)-1-phenylpropan-2-ol (12.0 mg, 0.0466 mmol) in methanol $(0.933 \mathrm{~mL})$ in a $20-\mathrm{mL}$, oven-dried reaction vial equipped with a magnetic stir bar under a nitrogen atmosphere was added hydrochloric acid $(0.068 \mathrm{~mL}, 12 \mathrm{~N}, 0.816 \mathrm{mmol}, 17.5$ equiv). The resulting solution was stirred for $1 \mathrm{~h}$ at room temperature, then diluted with methanol 
$(5 \mathrm{~mL})$ and concentrated under reduced pressure by rotary evaporation $\left(30{ }^{\circ} \mathrm{C}, \sim 20 \mathrm{~mm} \mathrm{Hg}\right)$ to yield $13.7 \mathrm{mg}$ of a pale-yellow solid. The solid was triturated with diethyl ether $(1.0 \mathrm{~mL})$ and filtered to yield $9.6 \mathrm{mg}(70 \%)$ of 7 as a white solid.

\section{Data for 7-i:}

1H NMR: $\left(500 \mathrm{MHz}, \mathrm{CDCl}_{3}\right)$

反 7.28-7.17 (m, $10 \mathrm{H}, \mathrm{HC}(1-3,10-12)), 4.43\left(\mathrm{~d}, J=11.9 \mathrm{~Hz}, 1 \mathrm{H}, \mathrm{H}_{2} \mathrm{C}(8)\right), 4.35$

(d, $\left.J=11.9 \mathrm{~Hz}, 1 \mathrm{H}, \mathrm{H}_{2} \mathrm{C}(8)\right), 4.28-4.16$ (br s, $3 \mathrm{H}, \mathrm{HO}, \mathrm{H}_{2} \mathrm{~N}$ ), 3.98 (d, $J=7.8$

$\mathrm{Hz}, 1 \mathrm{H}, \mathrm{HC}(5)), 3.79$ (ddd, $J=8.1,5.1,3.3 \mathrm{~Hz}, 1 \mathrm{H}, \mathrm{HC}(6)), 3.35$ (dd, $J=10.0$,

$\left.3.1 \mathrm{~Hz}, 1 \mathrm{H}, \mathrm{H}_{2} \mathrm{C}(7)\right), 3.23$ (dd, $\left.J=10.0,5.1 \mathrm{~Hz}, 1 \mathrm{H}, \mathrm{H}_{2} \mathrm{C}(7)\right)$.

${ }^{13} \mathrm{C}$ NMR: $\quad\left(126 \mathrm{MHz}, \mathrm{CDCl}_{3}\right)$

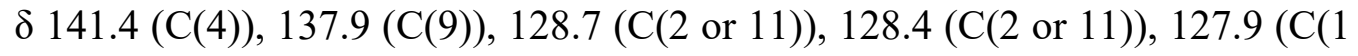

or 12)), $127.8(\mathrm{C}(10)), 127.7(\mathrm{C}(1$ or 12)), $127.1(\mathrm{C}(3)), 74.2(\mathrm{C}(6)), 73.4(\mathrm{C}(8))$,

$71.3(\mathrm{C}(7)), 57.7(\mathrm{C}(5))$.

$\underline{\text { IR: (neat) }}$

$3355(w), 3300(w), 3061(w), 3027(w), 3001(w), 2945(w), 2908$ (m), 2861

(w), 2740 (w), 2682 (w), 2112 (w), 1963 (w), 1897 (w), 1527 (w), 1495 (m), 1450 (m), 1384 (m), 1369 (w), 1353 (m), 1337 (w), 1323 (w), 1303 (w), 1247 (w), 1227 (w), 1204 (m), 1172 (w), 1159 (w), 1138 (m), 1118 (w), 1101 (m), 1084 (s), 1074 (m), 1038 (m), 1027 (m), 993 (m), 968 (m), 949 (m), 912 (m), 867 (w), $819(\mathrm{~m}), 804(\mathrm{~m}), 752(\mathrm{~m}), 742(\mathrm{~m}), 705(\mathrm{~s}), 697(\mathrm{~s}), 682(\mathrm{~m}), 648(\mathrm{~m}), 603$ (m), $589(\mathrm{w}), 539(\mathrm{~m}), 499(\mathrm{w}), 469(\mathrm{w}), 456(\mathrm{~m})$

HRMS: (ESI $\left.{ }^{+}\right)$Calcd for $\mathrm{C}_{16} \mathrm{H}_{20} \mathrm{NO}_{2}{ }^{+}: 258.1494$, found: 258.1490 . 
Analysis: $\quad \mathrm{C}_{16} \mathrm{H}_{19} \mathrm{NO}_{2}$

Calcd: C, 74.68\%; H, 7.44\%; N, 5.44\%

Found: C, 74.55\%; H, 7.16\%; N, 5.68\%

TLC: $\quad R_{f}=0.40(50: 50$ hexanes/ethyl acetate $)$ [UV/CAM]

Data for 7:

m.p: $\quad 147^{\circ} \mathrm{C}($ decomp)

1․ NMR: $\quad\left(500 \mathrm{MHz}, \mathrm{MeOH}-\mathrm{d}_{4}\right)$

$\delta$ 7.45-7.39 (m, $5 \mathrm{H}, \mathrm{HC}(1,2,11)), 7.34-7.24(\mathrm{~m}, 5 \mathrm{H}, 3,10,12)), 4.51(\mathrm{~d}, J=$ $\left.11.9 \mathrm{~Hz}, 1 \mathrm{H}, \mathrm{H}_{2} \mathrm{C}(8)\right), 4.40\left(\mathrm{~d}, J=11.9 \mathrm{~Hz}, 1 \mathrm{H}, \mathrm{H}_{2} \mathrm{C}(8)\right), 4.34(\mathrm{~d}, J=8.6 \mathrm{~Hz}, 1$ H, HC(5)), $4.06(\mathrm{dt}, J=8.0,3.7 \mathrm{~Hz}, 1 \mathrm{H}, \mathrm{HC}(6)), 3.44(\mathrm{dd}, J=10.4,3.2 \mathrm{~Hz}, 1 \mathrm{H}$, $\left.\mathrm{H}_{2} \mathrm{C}(7)\right), 3.28\left(\mathrm{dd}, J=10.3,4.3 \mathrm{~Hz}, 1 \mathrm{H}, \mathrm{H}_{2} \mathrm{C}(7)\right)$.

${ }^{13} \mathrm{C} \mathrm{NMR:} \quad\left(126 \mathrm{MHz}, \mathrm{MeOH}-\mathrm{d}_{4}\right)$

$\delta 139.1(\mathrm{C}(9)), 136.0(\mathrm{C}(4)), 130.6(\mathrm{C}(1$ or 12$)), 130.4(\mathrm{C}(2$ or 11$)), 129.4$ (C(10)), $129.0(\mathrm{C}(3)), 128.9(\mathrm{C}(2$ or 11$)), 128.8(\mathrm{C}(1$ or 12$)), 74.5(\mathrm{C}(8)), 72.8$ $(\mathrm{C}(6)), 71.9(\mathrm{C}(7)), 58.8(\mathrm{C}(5))$.

IR: (neat) $3226(\mathrm{~m}), 3021(\mathrm{w}), 2855(\mathrm{~m}), 2645(\mathrm{w}), 1945(\mathrm{w}), 1596(\mathrm{w}), 1507(\mathrm{~m}), 1498$ (m), $1458(\mathrm{w}), 1449(\mathrm{~m}), 1422(\mathrm{w}), 1372(\mathrm{w}), 1362(\mathrm{w}), 1335(\mathrm{w}), 1311(\mathrm{w})$, $1259(\mathrm{w}), 1241(\mathrm{w}), 1200(\mathrm{w}), 1188(\mathrm{w}), 1176(\mathrm{w}), 1141(\mathrm{w}), 1120(\mathrm{~m}), 1088$ (m), $1074(\mathrm{~m}), 1054(\mathrm{~m}), 1011(\mathrm{~m}), 967(\mathrm{w}), 924(\mathrm{~m}), 908(\mathrm{w}), 861(\mathrm{~m}), 804$ (w), $762(\mathrm{~m}), 746(\mathrm{~s}), 700(\mathrm{~s}), 627(\mathrm{~m}), 607(\mathrm{~m}), 590(\mathrm{~m}), 541(\mathrm{~m}), 491(\mathrm{w})$, $470(w)$

HRMS: $\quad\left(\mathrm{ESI}^{+}\right)$Calcd for $\mathrm{C}_{16} \mathrm{H}_{20} \mathrm{NO}_{2}^{+}: 258.1494$, found: 258.1487 . 
TLC: $\quad R_{f}=0.28(90: 10$ ethyl acetate/methanol) $[\mathrm{UV} / \mathrm{CAM}]$

\section{Preparation of $N$-((1R,2S)-3-(Benzyloxy)-2-hydroxy-1-phenylpropyl)-2-methylbenzamide}

\section{(5) from (1R,2S)-1-Amino-3-(benzyloxy)-1-phenylpropan-2-ol Hydrochloride (7) for}

\section{Evaluation of Enantiomeric Ratio.}

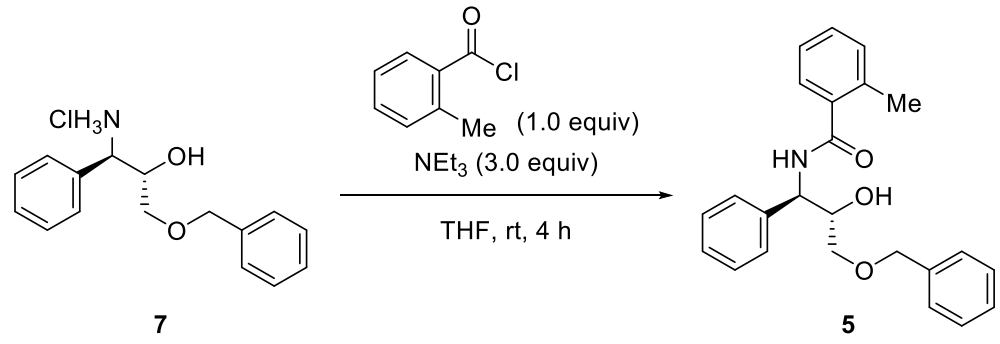

To a solution of (1R,2S)-1-Amino-3-(benzyloxy)-1-phenylpropan-2-ol hydrochloride 7 $(9.6 \mathrm{mg}, 33.0 \mu \mathrm{mol})$ in tetrahydrofuran $(0.5 \mathrm{~mL})$ in a $1-\mathrm{mL}$ conical vial was added triethylamine (13.8 $\mu \mathrm{L}, 99.0 \mu \mathrm{mol}, 3.0$ equiv) and (2-methyl)benzoyl chloride (4.3 $\mu \mathrm{L}, 33.0 \mu$ mol, 1.0 equiv). The resulting milky off-white suspension was stirred at $21^{\circ} \mathrm{C}$ for $4 \mathrm{~h}$. To the mixture was added dichloromethane $(0.5 \mathrm{~mL})$, and the solution was transferred to a $4-\mathrm{mL}$ vial. Water $(1.0 \mathrm{~mL})$ was added and the layers were separated. The aqueous layer was extracted with dichloromethane (3 X $1 \mathrm{~mL})$. The combined organic layers were dried over sodium sulfate $(0.5 \mathrm{~g})$, filtered, and concentrated under reduced pressure by rotary evaporation $\left(30{ }^{\circ} \mathrm{C}, \sim 20 \mathrm{~mm} \mathrm{Hg}\right)$. Column chromatography ( $1 \mathrm{~g}$ silica gel, $0.5 \mathrm{~cm}$ column) eluting with a gradient of hexanes/ethyl acetate, 90:10 to 50:50 (1-mL fractions) to yield $9.3 \mathrm{mg}(75 \%)$ of $N$-((1R,2S)-3-(Benzyloxy)-2hydroxy-1-phenylpropyl)-2-methylbenzamide (5) as a clear, colorless oil. Spectral data was consistent with our own internal data above.

CSP-HPLC: $\quad(R, S)-5 t_{R}=7.99 \min (91.3 \%),(S, R)-5 t_{R}=11.61 \min (8.7 \%)($ Regis $R, R$-WhelkO1, hexanes/i-PrOH, 70:30, $\left.1.0 \mathrm{~mL} / \mathrm{min} ; 220 \mathrm{~nm}, 24^{\circ} \mathrm{C}\right)$. 


\section{Preparation of (1R,2S)-3-(Benzyloxy)-1-(methyl(2-methylbenzyl)amino)-1-phenylpropan-2-} ol (9).

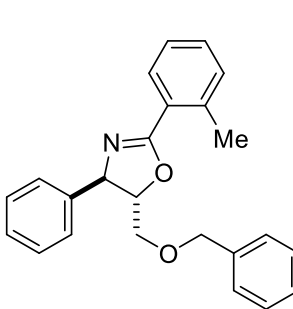

$2 \mathrm{~g}$

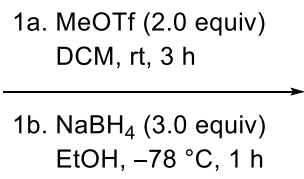

EtOH, $-78^{\circ} \mathrm{C}, 1 \mathrm{~h}$

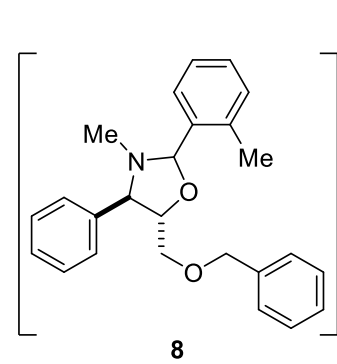

8

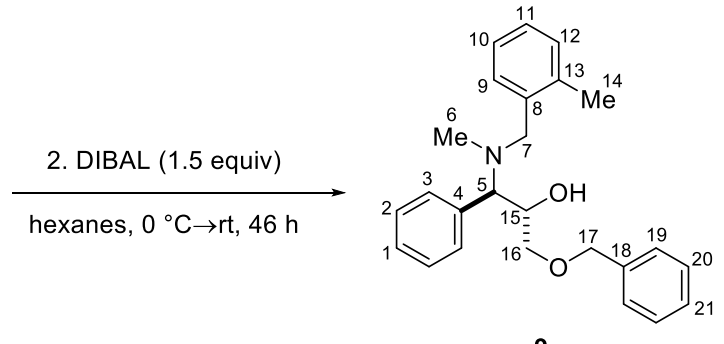

9

$1.0 \mathrm{mmol}$ reaction on racemic starting material for characterization

To a solution of \pm -trans-5-((benzyloxy)methyl)-2-(2-methylphenyl)-4-phenyl-4,5dihydrooxazole $(358 \mathrm{mg}, 1.0 \mathrm{mmol})$ in dichloromethane $(5.0 \mathrm{~mL}, 0.2 \mathrm{M})$ in a $25-\mathrm{mL}$ two-necked round-bottomed flask under an atmosphere of nitrogen was added methyl triflate $(226 \mu \mathrm{L}, 2.0$ mmol, 2.0 equiv). The clear, colorless solution was stirred at $21^{\circ} \mathrm{C}$ for $3 \mathrm{~h}$. The solution was cooled to $-78^{\circ} \mathrm{C}$ in a dry ice/iso-propanol bath and a solution of sodium borohydride $(114 \mathrm{mg}, 3.0 \mathrm{mmol}$, 3.0 equiv) in ethanol $(5.0 \mathrm{~mL}, 0.6 \mathrm{M})$ was added slowly (vigorous bubbling). After $1 \mathrm{~h}$ at $-78^{\circ} \mathrm{C}$, the reaction was quenched with a sat. aq. solution of ammonium chloride $(10 \mathrm{~mL})$ and transferred to a $50-\mathrm{mL}$ separatory funnel. Water $(2.5 \mathrm{~mL})$ and dichloromethane $(5.0 \mathrm{~mL})$ were added and the layers separated. The aqueous layer was extracted with dichloromethane $(10 \mathrm{~mL} \mathrm{X} \mathrm{2).} \mathrm{The}$ combined organic layers were dried over sodium sulfate $(5 \mathrm{~g})$, filtered and concentrated under reduced pressure by rotary evaporation $\left(30{ }^{\circ} \mathrm{C}, \sim 20 \mathrm{~mm} \mathrm{Hg}\right)$ to yield $425 \mathrm{mg}$ of 5(benzyloxy)methyl)-3-methyl-2-(2-methylphenyl)-4-phenyl-oxazolidine (8) as a clear, colorless oil.

To an anhydrous solution of the crude 5-(benzyloxy)methyl)-3-methyl-4-phenyl-2-(otolyl)oxazolidine from above in hexanes $(7.0 \mathrm{~mL}, 0.14 \mathrm{M})$ in a $50-\mathrm{mL}$ round-bottomed flask under nitrogen atmosphere at $0{ }^{\circ} \mathrm{C}$ in an ice water bath was added a solution of diisobutylaluminum 
hydride (1.0 M in hexanes, $1.5 \mathrm{~mL}, 1.5 \mathrm{mmol}, 1.5$ equiv). The resulting clear, colorless solution was allowed to warm to $21{ }^{\circ} \mathrm{C}$ and stirred for $46 \mathrm{~h}$. The resulting murky white suspension was cooled to $0{ }^{\circ} \mathrm{C}$ in an ice water bath, diluted with diethyl ether $(10 \mathrm{~mL})$, and quenched through a sequential addition of water $(60 \mu \mathrm{L})$, aq. solution of sodium hydroxide (2 M, $120 \mu \mathrm{L})$, and water $(150 \mu \mathrm{L})$. The milky suspension was warmed to $21^{\circ} \mathrm{C}$ and stirred for $15 \mathrm{~min}$. Magnesium sulfate ( $150 \mathrm{mg}$ ) was added. The resulting slurry was stirred at $21^{\circ} \mathrm{C}$ for $15 \mathrm{~min}$ and filtered through celite, washing with diethyl ether. The filtrate was concentrated under reduced pressure by rotary evaporation $\left(30^{\circ} \mathrm{C}, \sim 20 \mathrm{~mm} \mathrm{Hg}\right.$ ). The crude product was purified by column chromatography (50 g silica gel, $3.5 \mathrm{~cm}$ column) eluting with a gradient from hexanes to hexanes/ethyl acetate, 70:30 (25-mL fractions) to yield $323 \mathrm{mg}(86 \%)$ of 9 as a clear, colorless oil. A $65.6 \mathrm{mg}$ portion was further purified by Kugelrohr distillation $\left(110-120^{\circ} \mathrm{C}, 4.4 \times 10^{-5} \mathrm{~mm} \mathrm{Hg}\right)$ to afford $62.3 \mathrm{mg}$ of a clear colorless oil (95\% recovery). Quantitative ${ }^{1} \mathrm{H}$ NMR was conducted as described (page 54 ) with $13.7 \mathrm{mg}$ of 9 and $6.0 \mathrm{mg}$ of 1,1,2,2-tetrachloroethane (Molar ratio $=1.02$ ) to yield a purity of $100 \%$.

$\underline{0.2 \mathrm{mmol} \text { reaction on enantioenriched starting material for CSP-HPLC/optical rotation }}$

To a solution of (R,S)-5-((benzyloxy)methyl)-2-(2-methylphenyl)-4-phenyl-4,5dihydrooxazole $(72 \mathrm{mg}, 0.2 \mathrm{mmol})$ in dichloromethane $(1.0 \mathrm{~mL}, 0.2 \mathrm{M})$ in a 1-dram vial under an atmosphere of nitrogen was added methyl triflate ( $45 \mu \mathrm{L}, 0.4 \mathrm{mmol}, 2.0$ equiv). The clear, colorless solution was stirred at $21^{\circ} \mathrm{C}$ for $3 \mathrm{~h}$. The solution was cooled to $-78^{\circ} \mathrm{C}$ in a dry ice/iso-propanol bath and a solution of sodium borohydride $(23 \mathrm{mg}, 0.6 \mathrm{mmol}, 3.0$ equiv) in ethanol (1.0 mL, 0.6 M) was added slowly (vigorous bubbling). After $1 \mathrm{~h}$ at $-78{ }^{\circ} \mathrm{C}$, the reaction was quenched with a sat. aq. solution of ammonium chloride $(2 \mathrm{~mL})$ and transferred to a $5-\mathrm{mL}$ separatory funnel. Water $(2 \mathrm{~mL})$ and dichloromethane $(2 \mathrm{~mL})$ was added and the layers separated. The aqueous layer was 
extracted with dichloromethane $(2 \times 2 \mathrm{~mL})$. The combined organic layers were dried over sodium sulfate $(2 \mathrm{~g})$ and concentrated under reduced pressure by rotary evaporation $\left(30^{\circ} \mathrm{C}, \sim 20 \mathrm{~mm} \mathrm{Hg}\right)$ to yield $81 \mathrm{mg}$ of 5-(benzyloxy)methyl)-3-methyl-2-(2-methylphenyl)-4-phenyl-oxazolidine (8) as a clear, colorless oil.

To an anhydrous solution of the crude 5-(benzyloxy)methyl)-3-methyl-4-phenyl-2-(otolyl)oxazolidine from above in hexanes $(1.4 \mathrm{~mL}, 0.14 \mathrm{M})$ in a 1-dram vial under nitrogen atmosphere at $0{ }^{\circ} \mathrm{C}$ in an ice water bath was added a solution of diisobutylaluminum hydride (1.0 $\mathrm{M}$ in hexanes, $0.3 \mathrm{~mL}, 0.3 \mathrm{mmol}, 1.5$ equiv). The resulting clear, colorless solution was allowed to warm to $21{ }^{\circ} \mathrm{C}$ and stirred for $46 \mathrm{~h}$. The resulting murky white suspension was cooled to $0{ }^{\circ} \mathrm{C}$ in an ice water bath, diluted with diethyl ether $(0.5 \mathrm{~mL})$, and quenched through a sequential addition of water $(12 \mu \mathrm{L})$, aq. solution of sodium hydroxide $(2 \mathrm{M}, 24 \mu \mathrm{L})$, and water $(30 \mu \mathrm{L})$. The milky suspension was warmed to $21^{\circ} \mathrm{C}$ and stirred for $15 \mathrm{~min}$. Magnesium sulfate $(\sim 30 \mathrm{mg})$ was added. The resulting slurry was stirred at $21^{\circ} \mathrm{C}$ for $15 \mathrm{~min}$ and filtered through celite, washing with diethyl ether. The filtrate was concentrated under reduced pressure by rotary evaporation (30 ${ }^{\circ} \mathrm{C}, \sim 20 \mathrm{~mm} \mathrm{Hg}$ ). The crude product was purified by column chromatography (12 g silica gel, 0.5 $\mathrm{cm}$ column) eluting with a gradient from hexanes to hexanes/ethyl acetate, 80:20 (8-mL fractions) to yield $49 \mathrm{mg}(65 \%)$ of 9 as a clear, colorless oil.

\section{Data for 9:}

b.p. $\quad 110-120^{\circ} \mathrm{C}\left(4.4 \times 10^{-5} \mathrm{~mm} \mathrm{Hg}\right)$

1H NMR: $\quad\left(500 \mathrm{MHz}, \mathrm{CDCl}_{3}\right)$

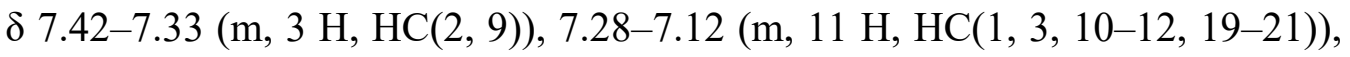
$4.49\left(\mathrm{~d}, J=12.2 \mathrm{~Hz}, 1 \mathrm{H}, \mathrm{H}_{2} \mathrm{C}(17)\right), 4.40\left(\mathrm{~d}, J=12.2 \mathrm{~Hz}, 1 \mathrm{H}, \mathrm{H}_{2} \mathrm{C}(17)\right), 4.30$ (ddd, $J=10.6,4.9,2.2 \mathrm{~Hz}, 1 \mathrm{H}, \mathrm{HC}(15)), 3.84$ (d, $J=10.6 \mathrm{~Hz}, 1 \mathrm{H}, \mathrm{HC}(5)), 3.53$ 
(d, $\left.J=13.0 \mathrm{~Hz}, 1 \mathrm{H}, \mathrm{H}_{2} \mathrm{C}(7)\right), 3.49$ (dd, $\left.J=10.6,2.2 \mathrm{~Hz}, 1 \mathrm{H}, \mathrm{H}_{2} \mathrm{C}(16)\right), 3.42$ (d, $\left.J=13.0 \mathrm{~Hz}, 1 \mathrm{H}, \mathrm{H}_{2} \mathrm{C}(7)\right), 3.26\left(\mathrm{dd}, J=10.6,5.0 \mathrm{~Hz}, 1 \mathrm{H}, \mathrm{H}_{2} \mathrm{C}(16)\right), 2.35$ (s, 3 $\left.\mathrm{H}, \mathrm{H}_{3} \mathrm{C}(14)\right), 2.10$ (s, $\left.3 \mathrm{H}, \mathrm{H}_{3} \mathrm{C}(6)\right)$.

${ }^{13} \mathrm{C} \mathrm{NMR:} \quad\left(126 \mathrm{MHz}, \mathrm{CDCl}_{3}\right)$

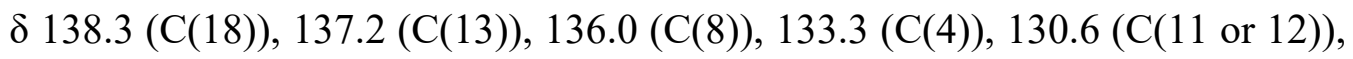
$130.0(\mathrm{C}(11$ or 12$)), 129.8(\mathrm{C}(3)), 128.2(\mathrm{C}(20)), 128.1(\mathrm{C}(2)), 128.0(\mathrm{C}(9))$, $127.4(\mathrm{C}(19)), 127.3(\mathrm{C}(21)), 127.3(\mathrm{C}(1)), 125.7(\mathrm{C}(10)), 73.3(\mathrm{C}(17)), 71.4$ (C(16)), $68.8(\mathrm{C}(5)), 68.6(\mathrm{C}(15)), 57.0(\mathrm{C}(7)), 35.7(\mathrm{C}(6)), 19.4(\mathrm{C}(14))$.

IR: (thin-film)

3394 (w, br), 3062 (w), 3028 (w), 2859 (m), 1603 (w), 1494 (m), 1453 (s), 1363 (w), $1284(w), 1200(w), 1118($ s), 1015 (m), $902(w), 878(w), 744(s), 703(s)$, $533(\mathrm{w})$.

HRMS: $\quad\left(\mathrm{ESI}^{+}\right)$Calcd for $\mathrm{C}_{25} \mathrm{H}_{30} \mathrm{NO}_{2}^{+}: 376.2277$, found: 376.2284 .

TLC: $\quad R_{f}=0.22(80: 20$ hexanes/ethyl acetate $)\left[\mathrm{UV} / \mathrm{KMnO}_{4}\right]$

CSP-HPLC: $\quad(S, R)-9 t_{R}=23.7 \min (5.3 \%),(R, S)-9 t_{R}=26.6 \min (94.7 \%)$ (Supelco Astec, hexanes/i-PrOH, 99:1, $2.0 \mathrm{~mL} / \mathrm{min} .220 \mathrm{~nm}, 24^{\circ} \mathrm{C}$ ).

Opt. Rot.: $[\alpha]_{\mathrm{D}}^{23} 8.4\left(c=0.52, \mathrm{CHCl}_{3}\right)$ 


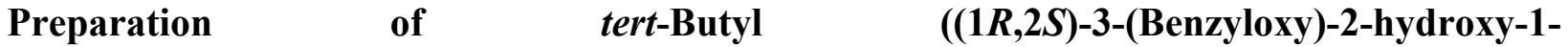

phenylpropyl)(methyl)carbamate (10).

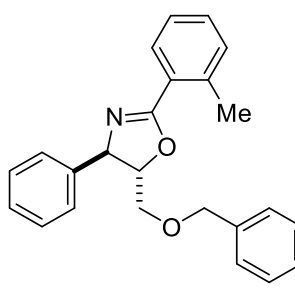

$2 \mathrm{~g}$ 1a. MeOTf (2.0 equiv)

$\mathrm{DCM}, \mathrm{rt}, 3 \mathrm{~h}$

1b. $\mathrm{NaBH}_{4}$ (3.0 equiv)

$\mathrm{EtOH},-78^{\circ} \mathrm{C}, 1 \mathrm{~h}$

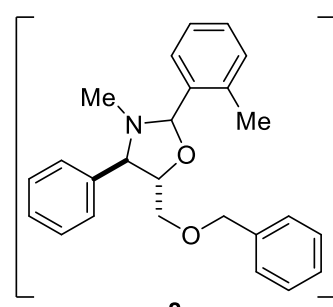

8

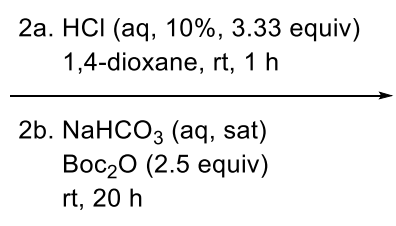

$\mathrm{rt}, 20 \mathrm{~h}$

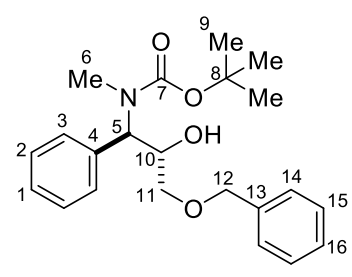

10

$1.0 \mathrm{mmol}$ reaction on racemic starting material for characterization

Formation of 5-(benzyloxy)methyl)-3-methyl-2-(2-methylphenyl)-4-phenyl-oxazolidine

(8) conducted exactly as above (in synthesis of 9) to yield $419 \mathrm{mg}$ of as a clear, colorless oil.

To a solution of the crude 5-(benzyloxy)methyl)-3-methyl-2-(2-methylphenyl)-4-phenyloxazolidine (8) from above in 1,4-dioxane $(6.5 \mathrm{~mL}, 0.15 \mathrm{M})$ in a 100 - $\mathrm{mL}$ round-bottomed flask was added an aq. solution of hydrochloric acid (1.0 M, $3.33 \mathrm{mmol}, 3.33$ equiv). The clear, colorless solution was stirred at $21{ }^{\circ} \mathrm{C}$ for $1 \mathrm{~h}$. To this solution was added a sat. aq. solution of sodium bicarbonate $(6.5 \mathrm{~mL})$, followed by solid sodium bicarbonate $(840 \mathrm{mg}, 10.0 \mathrm{mmol}, 10.0$ equiv) and di-tert-butyl dicarbonate (546 mg, $2.5 \mathrm{mmol}, 2.5$ equiv). The clear solution (with some insoluble solid sodium bicarbonate) was stirred at $21^{\circ} \mathrm{C}$ for $20 \mathrm{~h}$. To this mixture was added water $(5.0 \mathrm{~mL})$ to dissolve the excess sodium bicarbonate and the mixture transferred to a $125-\mathrm{mL}$ separatory funnel. The solution was extracted with ethyl acetate $(40 \mathrm{~mL} \mathrm{X} \mathrm{3).} \mathrm{The} \mathrm{combined} \mathrm{organic} \mathrm{layers}$ were dried over sodium sulfate $(5 \mathrm{~g})$ and concentrated under reduced pressure by rotary evaporation $\left(30^{\circ} \mathrm{C}, \sim 20 \mathrm{~mm} \mathrm{Hg}\right)$. The crude product was purified by column chromatography (60 g silica gel, $3.5 \mathrm{~cm}$ column) eluting with a gradient from hexanes to hexanes/ethyl acetate 65:35 (25-mL fractions) to yield $255 \mathrm{mg}(69 \%)$ of 10 as a clear, colorless oil. A $66.4 \mathrm{mg}$ portion was further purified by Kugelrohr distillation $\left(110-120{ }^{\circ} \mathrm{C}, 4.4 \times 10^{-5} \mathrm{~mm} \mathrm{Hg}\right)$ to afford $65.3 \mathrm{mg}$ of a clear colorless oil (98\% recovery). Quantitative ${ }^{1} \mathrm{H}$ NMR was conducted as described (page $S 4$ ) 
with $13.9 \mathrm{mg}$ of $\mathbf{1 0}$ and $6.2 \mathrm{mg}$ of 1,1,2,2-tetrachloroethane $($ Molar ratio $=1.00)$ to yield a purity of $99 \%$.

$\underline{0.2 \mathrm{mmol} \text { reaction on enantioenriched starting material for CSP-HPLC/optical rotation }}$

Formation of 5-(benzyloxy)methyl)-3-methyl-2-(2-methylphenyl)-4-phenyl-oxazolidine (8) conducted exactly as above (in synthesis of 9) to yield $79 \mathrm{mg}$ of as a clear, colorless oil.

To a solution of the crude 5-(benzyloxy)methyl)-3-methyl-2-(2-methylphenyl)-4-phenyloxazolidine $(8)$ from above in 1,4-dioxane $(1.3 \mathrm{~mL}, 0.15 \mathrm{M})$ in a $25-\mathrm{mL}$ round-bottomed flask was added an aq. solution of hydrochloric acid (1.0 M, $0.67 \mathrm{mmol}, 3.33$ equiv). The clear, colorless solution was stirred at $21{ }^{\circ} \mathrm{C}$ for $1 \mathrm{~h}$. To this solution was added a sat. aq. solution of sodium bicarbonate $(1.3 \mathrm{~mL})$, followed by solid sodium bicarbonate (168 mg, $2.0 \mathrm{mmol}, 10.0$ equiv) and di-tert-butyl dicarbonate (109 mg, $0.5 \mathrm{mmol}, 2.5$ equiv). The clear solution (with some insoluble solid sodium bicarbonate) was stirred at $21{ }^{\circ} \mathrm{C}$ for $20 \mathrm{~h}$. To this mixture was added water $(1.0 \mathrm{~mL})$ to dissolve the excess sodium bicarbonate and transferred to a $10-\mathrm{mL}$ separatory funnel. The solution was extracted with ethyl acetate $(10 \mathrm{~mL} \mathrm{X} \mathrm{3).} \mathrm{The} \mathrm{combined} \mathrm{organic} \mathrm{layers} \mathrm{were} \mathrm{dried}$ over sodium sulfate $(5 \mathrm{~g})$ and concentrated under reduced pressure by rotary evaporation $\left(30{ }^{\circ} \mathrm{C}\right.$, $\sim 20 \mathrm{~mm} \mathrm{Hg}$ ). The crude product was purified by column chromatography $(7 \mathrm{~g}$ silica gel, $0.5 \mathrm{~cm}$ column) eluting with a gradient from hexanes to hexanes/ethyl acetate 80:20 (8-mL fractions) to yield $57.1 \mathrm{mg}(77 \%)$ of $\mathbf{1 0}$ as a clear, colorless oil.

Data for 10:

b.p. $\quad 110-120^{\circ} \mathrm{C}\left(4.4 \times 10^{-5} \mathrm{~mm} \mathrm{Hg}\right)$

1․ NMR: $\quad\left(500 \mathrm{MHz}, \mathrm{CDCl}_{3}, 50^{\circ} \mathrm{C}\right)$

$\delta$ 7.34-7.21 (m, $10 \mathrm{H}, \mathrm{HC}(1-3)), 5.14(\mathrm{~d}, J=7.6 \mathrm{~Hz}, 1 \mathrm{H}, \mathrm{HC}(5)), 4.50(\mathrm{~d}, J=$ $\left.11.9 \mathrm{~Hz}, 1 \mathrm{H}, \mathrm{H}_{2} \mathrm{C}(12)\right), 4.47$ (d, $\left.J=11.9 \mathrm{~Hz}, 1 \mathrm{H}, \mathrm{H}_{2} \mathrm{C}(12)\right), 4.45-4.40$ (m, $1 \mathrm{H}$, 
$\mathrm{HC}(10)), 3.55\left(\mathrm{dd}, J=9.8,3.6 \mathrm{~Hz}, 1 \mathrm{H}, \mathrm{H}_{2} \mathrm{C}(11)\right), 3.43-3.37$ (m, $\left.1 \mathrm{H}, \mathrm{H}_{2} \mathrm{C}(11)\right)$, 2.78 (s, $\left.3 \mathrm{H}, \mathrm{H}_{3} \mathrm{C}(6)\right), 1.44$ (s, $\left.9 \mathrm{H}, \mathrm{H}_{3} \mathrm{C}(9)\right)$.

${ }^{13} \mathrm{C} \mathrm{NMR:} \quad\left(126 \mathrm{MHz}, \mathrm{CDCl}_{3}, 50{ }^{\circ} \mathrm{C}\right)$

$\delta 157.1(\mathrm{C}(7)$-rotomer1), $156.0(\mathrm{C}(7)$-rotomer2), $137.9(\mathrm{C}(4$ or 13$)), 137.8(\mathrm{C}(4$ or 13)), $128.5(\mathrm{C}(2$ or 15$)), 128.4(\mathrm{C}(2$ or 15$)), 128.1(\mathrm{C}(3)), 127.7(\mathrm{C}(14)), 127.7$ (C(1 or 16)), $127.6(\mathrm{C}(1$ or 16$)), 80.0(\mathrm{C}(8)), 73.4(\mathrm{C}(12)), 71.9(\mathrm{C}(11)), 70.1$ (C(10)-rotomer1), 69.7 (C(10)-rotomer2), 60.8 (C(5)-rotomer1), 60.4 (C(5)rotomer2), $32.4(\mathrm{C}(6)$-rotomer1), $28.4(\mathrm{C}(6)$-rotomer2), $28.5(\mathrm{C}(9))$.

IR: (thin-film)

3448 (w, br), 3031 (w), 2975 (w), 2930 (w), 1687 (s), 1479 (m), 1454 (m), 1391 (m), 1366 (m), 1327 (m), 1254 (w), 1147 (s), 1029 (w), 883 (w), 736 (m), 699 (m), $630(\mathrm{w})$.

HRMS: $\quad\left(\mathrm{ESI}^{+}\right)$Calcd for $\mathrm{C}_{22} \mathrm{H}_{29} \mathrm{NNaO}_{4}^{+}$: 394.1994, found: 394.1993.

TLC: $\quad R_{f}=0.13(80: 20$ hexanes/ethyl acetate $)\left[\mathrm{UV} / \mathrm{KMnO}_{4}\right]$

CSP-HPLC: $\quad(R, S)-\mathbf{1 0} t_{R}=8.6 \min (93.9 \%),(S, R)-10 t_{R}=10.3 \min (6.1 \%)$ (Supelco Astec, hexanes/i-PrOH, 90:10, 1.0mL/min; $\left.220 \mathrm{~nm}, 24{ }^{\circ} \mathrm{C}\right)$.

Opt. Rot.: $[\alpha]_{\mathrm{D}}^{23}-32.8\left(c=0.95, \mathrm{CHCl}_{3}\right)$

Preparation of (4R,5R)-2-(2-Bromophenyl)-4,5-diphenyl-4,5-dihydrooxazole (4u).

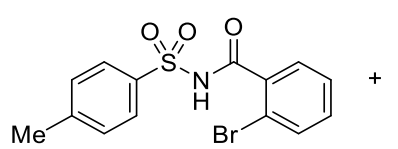

$1 \mathbf{k}$ (1.0 equiv)

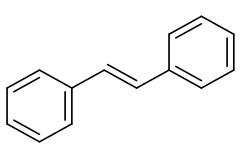

$2 r$ (1.2 equiv)

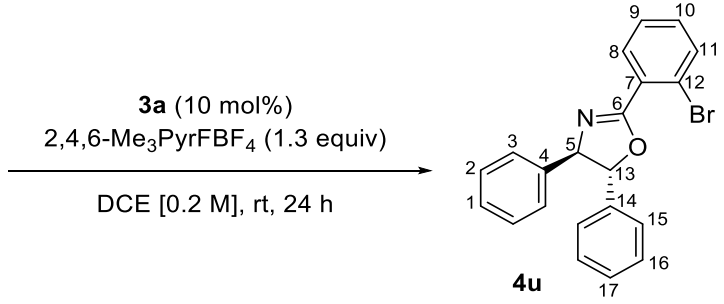

Following General Procedure I, 2-bromo- $N$-tosylbenzamide (354 mg, $1.00 \mathrm{mmol}$ ), 
diselenide catalyst $3 \mathbf{a}\left(82.1 \mathrm{mg}, 0.10 \mathrm{mmol}, 0.10\right.$ equiv), 2,4,6- $\mathrm{Me}_{3} \mathrm{PyF}^{+} \mathrm{BF}_{4}^{-}(295 \mathrm{mg}, 1.30 \mathrm{mmol}$, 1.30 equiv), and trans-stilbene (216 mg, $1.20 \mathrm{mmol}, 1.20$ equiv) were combined in 1,2-DCE (5.0 $\mathrm{mL}, 0.20 \mathrm{M}$ ) and allowed to stir for $24 \mathrm{~h}$ at $21^{\circ} \mathrm{C}$. The resulting suspension was worked up as described in the General Procedure and purified by column chromatography (100 g silica gel, 5.5 cm column) eluting with a gradient from hexanes to hexanes/ethyl acetate, $90: 10$ (25-mL fractions) to yield $316 \mathrm{mg}(83 \%)$ of $\mathbf{4 u}$ as a clear, yellow oil. A $58.2 \mathrm{mg}$ portion was further purified by Kugelrohr distillation $\left(130{ }^{\circ} \mathrm{C}, 4.0 \times 10^{-5} \mathrm{~mm} \mathrm{Hg}\right)$ to afford $54.9 \mathrm{mg}$ of a clear slightly yellow oil (94\% recovery).

\section{Data for $4 \mathbf{u}:$}

b.p. $130{ }^{\circ} \mathrm{C}\left(4.0 \times 10^{-5} \mathrm{~mm} \mathrm{Hg}\right)$

글 NMR: $\quad\left(500 \mathrm{MHz}, \mathrm{CDCl}_{3}\right)$

$\delta 7.90(\mathrm{~d}, J=7.5 \mathrm{~Hz}, 1 \mathrm{H}, \mathrm{HC}(8)), 7.73$ (d, $J=7.9 \mathrm{~Hz}, 1 \mathrm{H}, \mathrm{HC}(11)), 7.46-7.31$

(m, $12 \mathrm{H}, \mathrm{HC}(1-3,9-10,15-17)), 5.44$ (d, $J=8.0 \mathrm{~Hz}, 1 \mathrm{H}, \mathrm{HC}(13)), 5.32$ (d, $J$ $=8.0 \mathrm{~Hz}, 1 \mathrm{H}, \mathrm{HC}(5))$.

${ }^{13} \mathrm{C} \mathrm{NMR:} \quad\left(126 \mathrm{MHz}, \mathrm{CDCl}_{3}\right)$

$\delta 163.5(\mathrm{C}(6)), 141.6(\mathrm{C}(14)), 140.0(\mathrm{C}(4)), 134.0(\mathrm{C}(11)), 131.9(\mathrm{C}(10)), 131.6$ (C(8)), $129.5(\mathrm{C}(7)), 128.9(\mathrm{C}(16)), 128.8(\mathrm{C}(2)), 128.5(\mathrm{C}(17)), 127.8(\mathrm{C}(9))$, 127.2 (C(1)), $126.7(\mathrm{C}(3)), 125.9$ (C(15)), 122.1 (C(12)), 89.4 (C(13)), 79.1 $(\mathrm{C}(5))$.

IR: (thin-film)

$3063(\mathrm{w}), 3030$ (w), 2919 (w), 1651 (m), 1603 (w), 1589 (w), $1565(\mathrm{w}), 1495$ (w), $1475(\mathrm{w}), 1454(\mathrm{w}), 1432(\mathrm{w}), 1319(\mathrm{~m}), 1261(\mathrm{w}), 1221(\mathrm{w}), 1130(\mathrm{w})$, 1095 (m), 1025 (m), $964(\mathrm{~m}), 914(\mathrm{w}), 894(\mathrm{w}), 845(\mathrm{w}), 758(\mathrm{~s}), 731(\mathrm{~m}), 697$ 
(s), $653(\mathrm{w}), 640(\mathrm{w}), 604(\mathrm{w})$.

HRMS: $\quad\left(\mathrm{ESI}^{+}\right)$Calcd for $\mathrm{C}_{21} \mathrm{H}_{17} \mathrm{BrNO}^{+}:$378.0494, found: 378.0492 .

Analysis: $\quad \mathrm{C}_{21} \mathrm{H}_{16} \mathrm{BrNO}$

Calcd: C, 66.68\%; H, 4.26\%; N, 3.70\%

Found: C, 67.05\%; H, 4.15\%; N, 3.70\%

TLC: $\quad R_{f}=0.34\left(80: 20\right.$ hexanes/ethyl acetate) $\left[\mathrm{UV} / \mathrm{KMnO}_{4}\right]$

CSP-HPLC: $\quad(S, S)-\mathbf{4 u} t_{R}=8.2 \min (4.2 \%),(R, R)-4 \mathbf{u} t_{R}=9.0 \min (95.8 \%)$ (Daicel Chiralpak

IB-3, hexanes $/ i$-PrOH, 99:1, 1.0mL/min; $\left.220 \mathrm{~nm}, 24{ }^{\circ} \mathrm{C}\right)$.

Opt. Rot.: $[\alpha]_{\mathrm{D}}^{23} 32.6\left(c=1.00, \mathrm{CHCl}_{3}\right)$

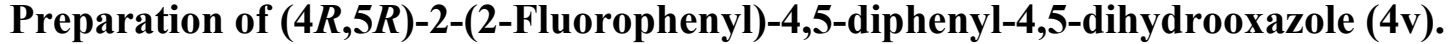

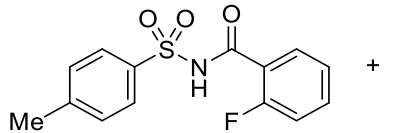

11 (1.0 equiv)

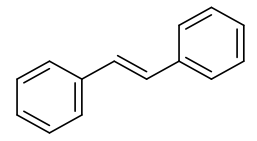

$2 r$ (1.2 equiv)

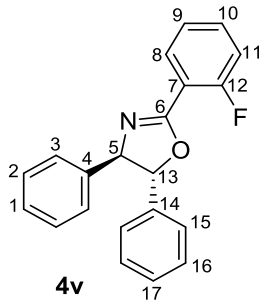

Following General Procedure I, 2-fluoro- $N$-tosylbenzamide (293 mg, $1.00 \mathrm{mmol}$ ), diselenide catalyst 3a (82.1 mg, $0.10 \mathrm{mmol}, 0.10$ equiv), 2,4,6- $\mathrm{Me}_{3} \mathrm{PyF}^{+} \mathrm{BF}_{4}^{-}(295 \mathrm{mg}, 1.30 \mathrm{mmol}$, 1.30 equiv), and trans-stilbene (216 mg, $1.20 \mathrm{mmol}, 1.20$ equiv) were combined in 1,2-DCE (5.0 $\mathrm{mL}, 0.20 \mathrm{M}$ ) and allowed to stir for $24 \mathrm{~h}$ at $21^{\circ} \mathrm{C}$. The resulting suspension was worked up as described in the General Procedure and purified by column chromatography (100 g silica gel, 5.5 $\mathrm{cm}$ column) eluting with a gradient from hexanes to hexanes/ethyl acetate, 90:10 (25-mL fractions) to yield $203 \mathrm{mg}(64 \%)$ of $4 \mathrm{v}$ as a clear, yellow oil. A $52.2 \mathrm{mg}$ portion was further purified by Kugelrohr distillation $\left(120-125^{\circ} \mathrm{C}, 3.5 \times 10^{-5} \mathrm{~mm} \mathrm{Hg}\right)$ to afford $52.4 \mathrm{mg}$ of a clear slightly yellow oil (100\% recovery). 


\section{Data for $4 \mathbf{v}$ :}

b.p. $\quad 120-125^{\circ} \mathrm{C}\left(3.5 \times 10^{-5} \mathrm{~mm} \mathrm{Hg}\right)$

1H NMR: $\quad\left(500 \mathrm{MHz}, \mathrm{CDCl}_{3}\right)$

$\delta 8.08(\mathrm{td}, J=7.5,1.8 \mathrm{~Hz}, 1 \mathrm{H}, \mathrm{HC}(9)), 7.56-7.50(\mathrm{~m}, 1 \mathrm{H}, \mathrm{HC}(8)), 7.45-7.31$

(m, $10 \mathrm{H}, \mathrm{HC}(1-3,15-17)), 7.29-7.20$ (m, $2 \mathrm{H}, \mathrm{HC}(10,11)), 5.44$ (d, J= 7.6 Hz, $1 \mathrm{H}, \mathrm{HC}(13)), 5.30(\mathrm{~d}, J=7.6 \mathrm{~Hz}, 1 \mathrm{H}, \mathrm{HC}(5))$.

${ }^{13} \mathrm{C}$ NMR: $\quad\left(126 \mathrm{MHz}, \mathrm{CDCl}_{3}\right)$

$\delta 161.5\left(\mathrm{~d},{ }^{1} J_{C-F}=258.7 \mathrm{~Hz}, \mathrm{C}(12)\right), 160.9\left(\mathrm{~d},{ }^{3} J_{C-F}=5.3 \mathrm{~Hz}, \mathrm{C}(6)\right), 141.8$ (C(14)), $140.3(\mathrm{C}(4)), 133.2\left(\mathrm{~d},{ }^{3} J_{C-F}=8.7 \mathrm{~Hz}, \mathrm{C}(8)\right), 131.4(\mathrm{C}(9)), 128.9$ (C(16)), $128.8(\mathrm{C}(2)), 128.4(\mathrm{C}(17)), 127.8(\mathrm{C}(1)), 126.7(\mathrm{C}(3)), 125.6(\mathrm{C}(15))$, $124.0\left(\mathrm{~d},{ }^{3} J_{C-F}=3.8 \mathrm{~Hz}, \mathrm{C}(10)\right), 116.8\left(\mathrm{~d},{ }^{2} J_{C-F}=21.7 \mathrm{~Hz}, \mathrm{C}(11)\right), 115.9\left(\mathrm{~d},{ }^{2} J_{C-}\right.$ $\left.{ }_{F}=10.0 \mathrm{~Hz}, \mathrm{C}(7)\right)$.

${ }^{19} \mathrm{~F}$ NMR: $\quad\left(471 \mathrm{MHz}, \mathrm{CDCl}_{3}\right)$

$\delta-108.4(\mathrm{~s}, \mathrm{FC}(12))$

IR: (thin-film)

3064 (w), 3031 (w), 2921 (w), 1647 (s), 1613 (m), 1584 (w), 1496 (m), $1456(\mathrm{~m})$, $1323(\mathrm{~m}), 1269$ (m), 1232 (m), $1158(\mathrm{w}), 1111(\mathrm{~m}), 1075(\mathrm{w}), 1054(\mathrm{~m}), 1030$ (m), $968(\mathrm{~m}), 915(\mathrm{w}), 895(\mathrm{w}), 820(\mathrm{w}), 760(\mathrm{~s}), 745(\mathrm{~m}), 698(\mathrm{~s}), 670(\mathrm{w}), 604$ (w).

HRMS: (ESI ${ }^{+}$Calcd for $\mathrm{C}_{21} \mathrm{H}_{17} \mathrm{FNO}^{+}:$318.1294, found: 318.1291 .

Analysis: $\mathrm{C}_{21} \mathrm{H}_{16} \mathrm{FNO}$

Calcd: C, 79.48\%; H, 5.08\%; N, 4.41\%

Found: C, 79.31\%; H, 4.90\%; N, 4.37\% 
TLC: $\quad R_{f}=0.34(80: 20$ hexanes/ethyl acetate $)\left[\mathrm{UV} / \mathrm{KMnO}_{4}\right]$

CSP-HPLC: $\quad(R, R)-4 \mathbf{v} t_{R}=6.0 \mathrm{~min}(92.0 \%),(S, S)-4 \mathbf{v} t_{R}=12.6 \mathrm{~min}(8.0 \%)($ Regis $R, R$-Whelk-

O1, hexanes/i-PrOH, 70:30, $\left.1.0 \mathrm{~mL} / \mathrm{min} ; 220 \mathrm{~nm}, 24^{\circ} \mathrm{C}\right)$.

Opt. Rot.: $[\alpha]_{\mathrm{D}}{ }^{23} 21.0\left(c=0.50, \mathrm{CHCl}_{3}\right)$

\section{Preparation of (4R,5R)-2-(2-(Diphenylphosphaneyl)phenyl)-4,5-diphenyl-4,5- dihydrooxazole (11).}

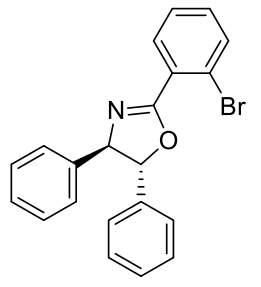

4u

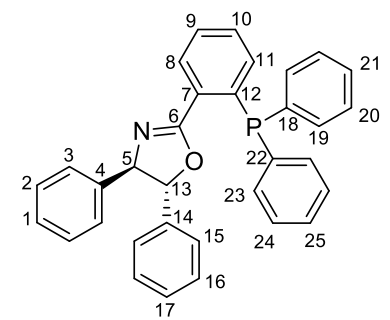

11

To an anhydrous suspension of copper(I) iodide (14.3 $\mathrm{mg}, 0.075 \mathrm{mmol}, 0.125$ equiv) in toluene $(2.4 \mathrm{~mL})$ in a $15 \mathrm{~mL}$ one-piece reflux condenser-round-bottomed flask was added $N, N^{\prime}-$ dimethylethylenediamine ( $56 \mu \mathrm{L}, 0.525 \mathrm{mmol}, 0.875$ equiv). The resulting bright blue suspension was added diphenylphosphine (196 $\mu \mathrm{L}, 1.128 \mathrm{mmol}, 1.88$ equiv). The mixture was stirred at 21 ${ }^{\circ} \mathrm{C}$ for $20 \mathrm{~min}$ and the blue color dissipated. To the mixture was added cesium carbonate (733 $\mathrm{mg}$, $2.25 \mathrm{mmol}, 3.75 \mathrm{mmol})$, followed by a solution of (4R,5R)-2-(2-bromophenyl)-4,5-diphenyl-4,5dihydrooxazole $4 \mathbf{u}(227 \mathrm{mg}, 0.6 \mathrm{mmol})$ in toluene $(2.4 \mathrm{~mL}, 0.25 \mathrm{M})$. The resulting murky yellow mixture was heated to $110{ }^{\circ} \mathrm{C}$ in an oil bath and stirred for $9 \mathrm{~h}$ (reaction became orange at $110{ }^{\circ} \mathrm{C}$ ). The reaction was cooled to $21^{\circ} \mathrm{C}$ and filtered through celite, washing with dichloromethane. The filtrate was concentrated under reduced pressure by rotary evaporation $\left(30^{\circ} \mathrm{C}, \sim 20 \mathrm{~mm} \mathrm{Hg}\right)$. The crude product was purified by column chromatography (50 g silica gel, $3.5 \mathrm{~cm}$ column) eluting with a gradient from hexanes to hexanes/ethyl acetate, 92.5:7.2 (25-mL fractions) to yield $201 \mathrm{mg}$ 
(69\%) of 11 as a pearly foam. A $63.0 \mathrm{mg}$ portion was further purified by Kugelrohr distillation $\left(175-180^{\circ} \mathrm{C}, 7.0 \times 10^{-5} \mathrm{~mm} \mathrm{Hg}\right)$ to afford a glassy colorless solid.

\section{$\underline{\text { Data for 11: }}$}

b.p. $\quad 175-180^{\circ} \mathrm{C}\left(7.0 \times 10^{-5} \mathrm{~mm} \mathrm{Hg}\right)$

1H NMR: $\quad\left(500 \mathrm{MHz}, \mathrm{CDCl}_{3}\right)$

$\delta 8.13(\mathrm{dd}, J=7.8,3.6 \mathrm{~Hz}, 1 \mathrm{H}, \mathrm{HC}(11)), 7.41(\mathrm{t}, J=7.5 \mathrm{~Hz}, 1 \mathrm{H}, \mathrm{HC}(1$ or 17$))$, 7.37-7.27 (m, $14 \mathrm{H}, \mathrm{HC}(2,9,10,16,19,21,23,25,20$ or 24$)), 7.22-7.17$ (m, 5 $\mathrm{H}, \mathrm{HC}(3,20$ or 24,1 or 17$)), 6.96(\mathrm{dd}, J=7.8,4.0 \mathrm{~Hz}, 1 \mathrm{H}, \mathrm{HC}(8)), 6.89$ (dd, $J$ $=7.1,2.7 \mathrm{~Hz}, 2 \mathrm{H}, \mathrm{HC}(15)), 5.15(\mathrm{~d}, J=9.0 \mathrm{~Hz}, 1 \mathrm{H}, \mathrm{HC}(13)), 5.11(\mathrm{~d}, J=9.0$ $\mathrm{Hz}, 1 \mathrm{H}, \mathrm{HC}(5))$.

${ }^{13} \mathrm{C} \mathrm{NMR:} \quad\left(126 \mathrm{MHz}, \mathrm{CDCl}_{3}\right)$

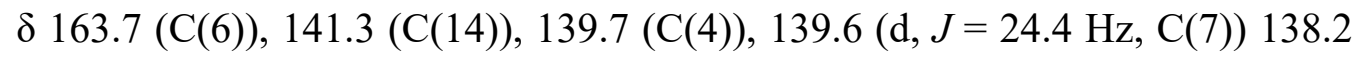
(d, $J=13.0 \mathrm{~Hz}, \mathrm{C}(18$ or 22$)), 138.0(\mathrm{~d}, J=10.6 \mathrm{~Hz}, \mathrm{C}(18$ or 22$)), 134.4(\mathrm{~d}, J=$ 21.1 Hz, C(19 or 23)), 134.0 (C(8)), $133.9(\mathrm{~d}, J=20.4 \mathrm{~Hz}, \mathrm{C}(19$ or 23$)), 131.1$ (d, $J=19.3 \mathrm{~Hz}, \mathrm{C}(12)), 130.9$ (C(9 or 10$)), 130.4(\mathrm{~d}, J=2.9 \mathrm{~Hz}, \mathrm{C}(11)), 128.7$ (C(2)), $128.7(\mathrm{C}(21$ or 25$)), 128.6(\mathrm{C}(21$ or 25$)), 128.5(\mathrm{~d}, J=1.0 \mathrm{~Hz}, \mathrm{C}(20$ or 24)), $128.4(\mathrm{C}(20$ or 24$)), 128.4(\mathrm{C}(16)), 128.3(\mathrm{C}(9$ or 10$)), 128.1$ (C(1 or 17$))$, $127.2(\mathrm{C}(1$ or 17$)), 126.6(\mathrm{C}(15)), 126.1(\mathrm{C}(3))$.

1ㅏ NMR: $\quad\left(202.5 \mathrm{MHz}, \mathrm{CDCl}_{3}\right)$

$\delta-5.7(\mathrm{~s}, \mathrm{PC}(12,18,22))$

IR: (thin-film)

$3063(\mathrm{w}), 1649$ (m), 1454 (w), 1434 (m), $1319(\mathrm{w}), 1274(\mathrm{w}), 1089$ (m), 1044 (m), $968(\mathrm{w}), 911(\mathrm{w}), 743(\mathrm{~m}), 696(\mathrm{~s})$. 
HRMS: $\quad\left(\mathrm{ESI}^{+}\right)$Calcd for $\mathrm{C}_{33} \mathrm{H}_{27} \mathrm{NOP}^{+}$: 484.1380, found: 484.1822 .

Analysis: $\quad \mathrm{C}_{33} \mathrm{H}_{26} \mathrm{NOP}$

Calcd: C, 81.97\%; H, 5.42\%; N, 2.90\%

Found: C, 81.86\%; H, 5.19\%; N, 3.25\%

TLC: $\quad R_{f}=0.23(90: 10$ hexanes/ethyl acetate) [UV/KMnO 4$]$

CSP-SFC: $\quad(R, R)-11 t_{R}=13.2 \min (97.3 \%),(S, S)-11 t_{R}=16.2 \min (2.8 \%)($ Chiralpak AD-H, $\left.\mathrm{CO}_{2} / \mathrm{MeOH}, 95: 5,2.0 \mathrm{~mL} / \mathrm{min} ; 254 \mathrm{~nm}, 40{ }^{\circ} \mathrm{C}\right)$.

Opt. Rot.: $[\alpha]_{\mathrm{D}}^{23}-7.5\left(c=0.88, \mathrm{CHCl}_{3}\right)$

Preparation of (4R,5R)-2-(2-(Diphenylphosphinyl)phenyl)-4,5-diphenyl-4,5-dihydrooxazole.

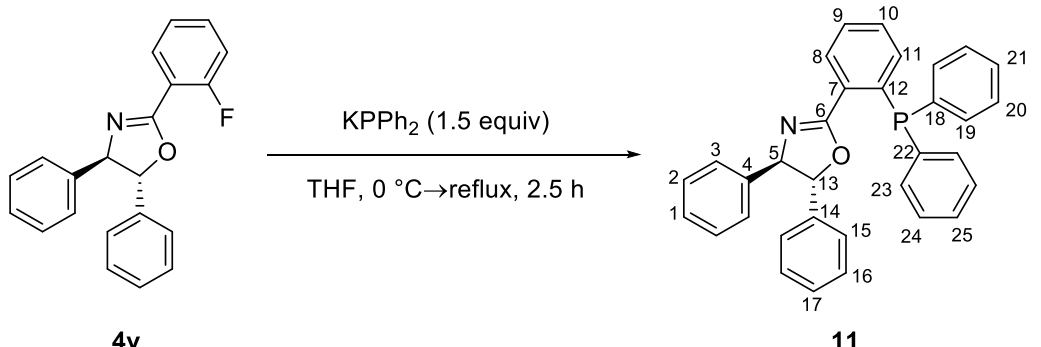

To an anhydrous slurry of potassium hydride (27 mg, $0.675 \mathrm{mmol}, 1.5$ equiv) in tetrahydrofuran $(1.4 \mathrm{~mL}, 0.48 \mathrm{M})$ in a 2-neck conical flask under an atmosphere of argon at $0{ }^{\circ} \mathrm{C}$ in an ice bath was added dropwise diphenylphosphine $(118 \mu \mathrm{L}, 0.675 \mathrm{mmol}, 1.5$ equiv). (The slurry slowly turned from colorless to yellow to orange as the addition proceeded.)

To an anhydrous solution of (4R,5R)-2-(2-fluorophenyl)-4,5-diphenyl-4,5-dihydrooxazole (143 $\mathrm{mg}, 0.45 \mathrm{mmol})$ in tetrahydrofuran $(1.0 \mathrm{~mL}, 1.0 \mathrm{M})$ in a $5 \mathrm{~mL}$ one-piece reflux condenserround-bottomed flask under argon was added the solution of potassium diphenylphosphide (freshly synthesized above) via cannula transfer at $0{ }^{\circ} \mathrm{C}$ in an ice bath. The resulting red solution was stirred at $21^{\circ} \mathrm{C}$ for $10 \mathrm{~min}$, the set to reflux at $70^{\circ} \mathrm{C}$ in an oil bath. After $2.5 \mathrm{~h}$, the mixture was cooled to 
$21{ }^{\circ} \mathrm{C}$, diluted with dichloromethane $(15 \mathrm{~mL})$, and poured onto a sat. aq. solution of sodium bicarbonate $(15 \mathrm{~mL})$. The mixture was transferred to a $60-\mathrm{mL}$ separatory funnel and the layers were separated. The organic layer was washed with water $(15 \mathrm{~mL})$ and dried over sodium sulfate (5 g) and concentrated under reduced pressure by rotary evaporation $\left(30^{\circ} \mathrm{C}, \sim 20 \mathrm{~mm} \mathrm{Hg}\right.$ ). The crude product was purified by column chromatography (50 g silica gel, $3.5 \mathrm{~cm}$ column) eluting with a gradient from hexanes to hexanes/ethyl acetate, 92.5:7.5 (25-mL fractions) to yield $96 \mathrm{mg}$ (44\%) of 11 as a clear oil.

$\underline{\text { Data for 11: }}$

CSP-SFC: $\quad(R, R)-\mathbf{1 1} t_{R}=13.2 \min (95.2 \%),(S, S)-11 t_{R}=16.2 \min (4.8 \%)($ Chiralpak AD-H, $\left.\mathrm{CO}_{2} / \mathrm{MeOH}, 95: 5,2.0 \mathrm{~mL} / \mathrm{min} ; 254 \mathrm{~nm}, 40^{\circ} \mathrm{C}\right)$. 


\section{Substrates Displaying Poor Conversion to the Desired Oxazoline}

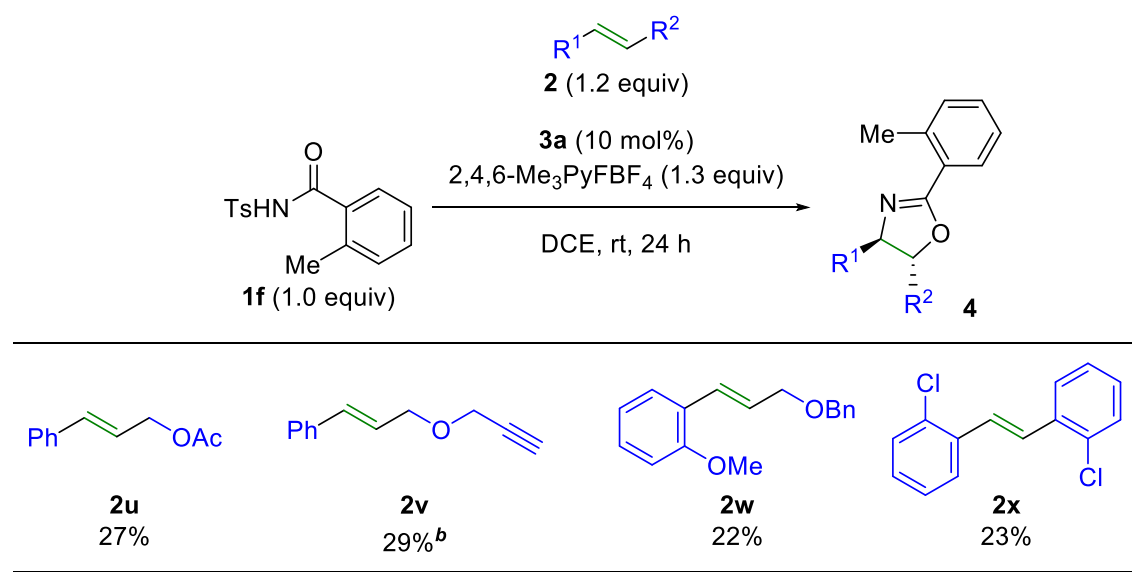

Following a modification of General Procedure I, 2-methyl- $N$-tosylbenzamide $(57.9 \mathrm{mg}$, $0.20 \mathrm{mmol}$ ), diselenide catalyst $3 \mathbf{a}$ (16.4 mg, $0.02 \mathrm{mmol}, 0.10$ equiv), $2,4,6-\mathrm{Me}_{3} \mathrm{PyF}^{+} \mathrm{BF}_{4}^{-}(59.0$ $\mathrm{mg}, 0.26 \mathrm{mmol}, 1.30$ equiv), 1,1,2,2-tetrachloroethane ( $21.1 \mu \mathrm{L}, 0.20 \mathrm{mmol}, 1.00$ equiv), and the corresponding alkene (0.24 mmol, 1.20 equiv) were combined in 1,2-DCE $(1.0 \mathrm{~mL}, 0.20 \mathrm{M})$ and allowed to stir for $24 \mathrm{~h}$ at $21{ }^{\circ} \mathrm{C}$. The resulting suspension was worked up as described in the General Procedure.

\section{Purification:}

For 2u: Purified by column chromatography on Teledyne ISCO Combi-Flash (silica gel, 25 g Luknova SuperSep column) eluting with a gradient of hexanes/ethyl acetate (20-mL fractions). Prep TLC run with dichloromethane to yield $16.4 \mathrm{mg}(27 \%)$ as a colorless oil.

For 2v: (Run with di(2,4-dimthoxyphenyl) diselenide as the catalyst.) Purified by column chromatography (50 g silica gel, $3.5 \mathrm{~cm}$ column) eluting with a gradient from hexanes to hexanes/ethyl acetate, 90:10 (20-mL fractions) to yield $17.5 \mathrm{mg}(29 \%)$ as a colorless oil.

$\underline{\text { For } \mathbf{2 w}}$ : Purified by column chromatography (50 g silica gel, $3.5 \mathrm{~cm}$ column) eluting with a gradient from hexanes to hexanes/ethyl acetate, $85: 15$ (20-mL fractions) to yield $17.1 \mathrm{mg}(22 \%)$ as a clear, slightly yellow oil that decomposed on the order of days. 
For 2x: Purified by column chromatography on Teledyne ISCO Combi-Flash (silica gel, 25 g Luknova SuperSep column) eluting with hexanes/acetone, 90:10. Prep TLC with dichloromethane to yield $17.8 \mathrm{mg}(23 \%)$ as a colorless oil.

\section{Substrates Displaying No Conversion to the Desired Oxazoline}
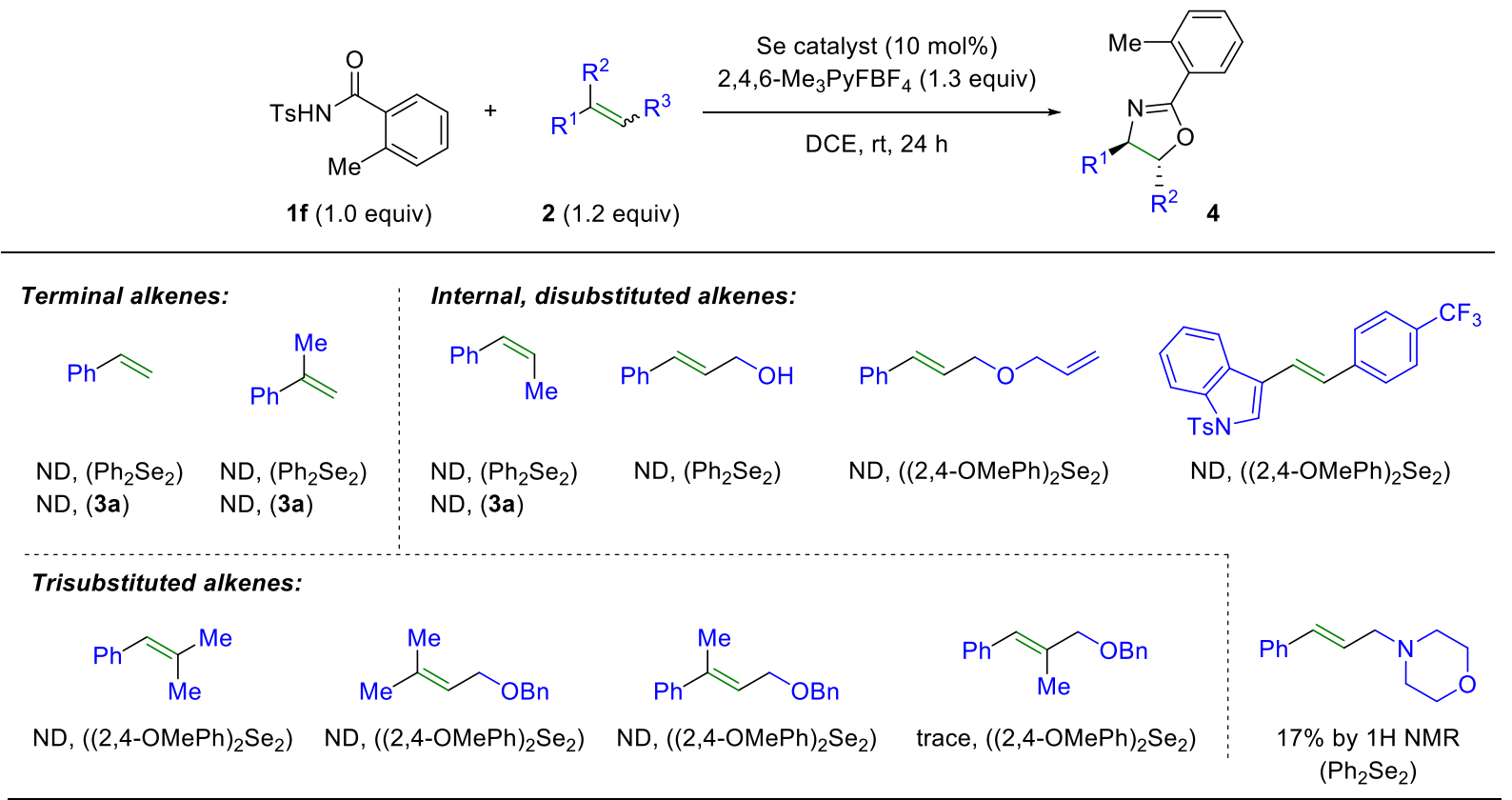

Following a modification of General Procedure I, 2-methyl- $N$-tosylbenzamide $(57.9 \mathrm{mg}$, $0.20 \mathrm{mmol}$ ), diselenide catalyst (0.05 mmol, 0.25 equiv), 2,4,6- $\mathrm{Me}_{3} \mathrm{PyF}^{+} \mathrm{BF}_{4}^{-}$(59.0 mg, 0.26 mmol, 1.30 equiv), 1,1,2,2-tetrachloroethane $(21.1 \mu \mathrm{L}, 0.20 \mathrm{mmol}, 1.00$ equiv), and the corresponding alkene $(0.24 \mathrm{mmol}, 1.20$ equiv) were combined in 1,2-DCE $(1.0 \mathrm{~mL}, 0.20 \mathrm{M})$ and allowed to stir for $24 \mathrm{~h}$ at $21{ }^{\circ} \mathrm{C}$. The resulting suspension was worked up as described in the General Procedure.

\section{Observations:}

For the following alkenes, starting material was partially consumed but no product was observed. styrene, $\alpha$-methylstyrene, $(Z)$ - $\beta$-methylstyrene 
For the following alkenes, starting material was fully consumed but no product was observed. styrene, $\alpha$-methylstyrene, (Z)- $\beta$-methylstyrene, cinnamyl alcohol, allyl cinnamyl alcohol, $(E)-N$ tosyl-3-(4-(trifluoromethyl)styryl)-1H-indole, $\beta, \beta$-dimethylstyrene, 3-benzyloxy-1,1dimethylpropene, and (E)-3-benzyloxy-1-methyl-1-phenylpropene For the following alkene, starting was material fully consumed but only a trace $(<5 \%)$ amount of product was observed by $\underline{1} \underline{\underline{H}}$ NMR analysis. (E)-3-benzyloxy-2-methyl-1-phenylpropene

For the following alkene, starting material was fully consumed and $17 \%$ yield by of product was found by $\underline{1} \underline{\mathrm{H}}$ NMR analysis.

$N$-cinnamylmorpholine

\section{Reaction Kinetics Comparison for (E)-4-Octene (Figure 3)}

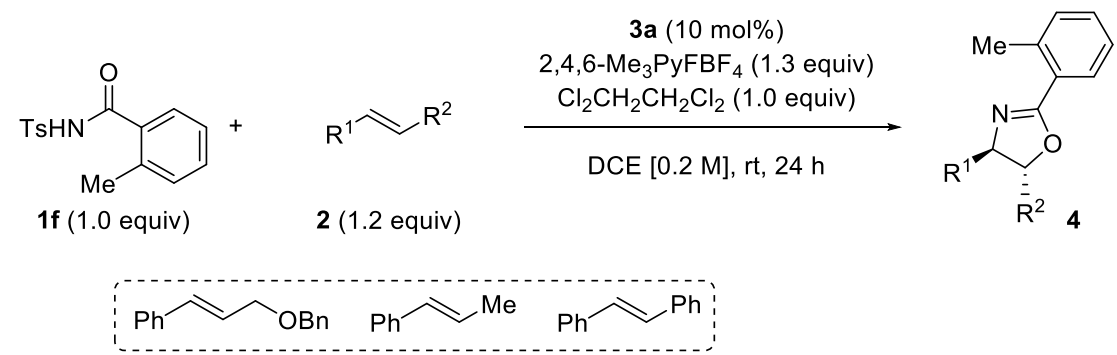

Following a modification of General Procedure I, 2-methyl- $N$-tosylbenzamide (57.9 mg, $0.20 \mathrm{mmol}$ ), diselenide catalyst 3a (16.4 mg, $0.02 \mathrm{mmol}, 0.10$ equiv), 2,4,6- $\mathrm{Me}_{3} \mathrm{PyF}^{+} \mathrm{BF}_{4}^{-}(59.0$ $\mathrm{mg}, 0.26 \mathrm{mmol}, 1.30$ equiv), 1,1,2,2-tetrachloroethane ( $21.1 \mu \mathrm{L}, 0.20 \mathrm{mmol}, 1.00$ equiv), and the corresponding alkene ( $0.24 \mathrm{mmol}, 1.20$ equiv) were combined in 1,2-DCE $(1.0 \mathrm{~mL}, 0.20 \mathrm{M})$ and allowed to stir for $24 \mathrm{~h}$ at $2{ }^{\circ} \mathrm{C}$. At the marked timepoints, a $50 \mu \mathrm{L}$ aliquot was removed from the reaction, added to $0.4 \mathrm{~mL} \mathrm{CDCl}_{3}$ and analyzed for yield by ${ }^{1} \mathrm{H}$ NMR by comparison of peak integrations (solvent suppression of DCE peak, 10 satpwr, 8 scans, 5 second relaxation delay). 

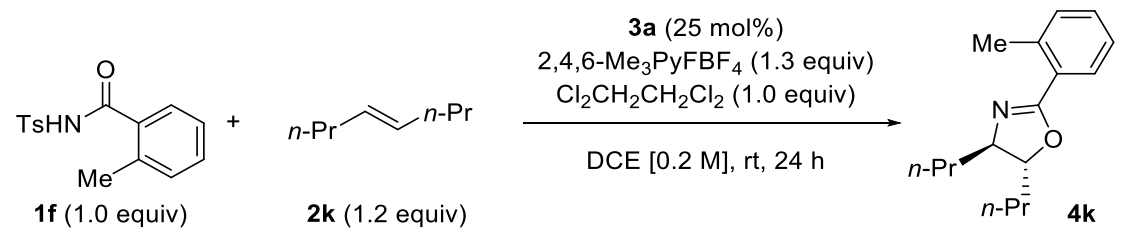

Following a modification of General Procedure I, 2-methyl- $N$-tosylbenzamide (57.9 mg, $0.20 \mathrm{mmol}$ ), diselenide catalyst 3a (16.4 mg, $0.02 \mathrm{mmol}, 0.10$ equiv), 2,4,6- $\mathrm{Me}_{3} \mathrm{PyF}^{+} \mathrm{BF}_{4}^{-}(59.0$ $\mathrm{mg}, 0.26 \mathrm{mmol}, 1.30$ equiv), 1,1,2,2-tetrachloroethane ( $21.1 \mu \mathrm{L}, 0.20 \mathrm{mmol}, 1.00$ equiv), and the trans-4-octene ( $26.9 \mu \mathrm{L}, 0.24 \mathrm{mmol}, 1.20$ equiv) were combined in 1,2-DCE $(1.0 \mathrm{~mL}, 0.20 \mathrm{M})$ (alkene added neat) and allowed to stir for $24 \mathrm{~h}$ at $21^{\circ} \mathrm{C}$. At the marked timepoints, a $50 \mu \mathrm{L}$ aliquot was removed from the reaction, added to $0.4 \mathrm{~mL} \mathrm{CDCl}_{3}$ and analyzed for yield by ${ }^{1} \mathrm{H} \mathrm{NMR}$ by comparison of peak integrations (solvent suppression of DCE peak, 10 satpwr, 8 scans, 5 second relaxation delay). 


\section{References}

1. Dieter, R. K.; Silks, L. A.; Fishpaugh, J. A.; Kastner, M. E., J. Am. Chem. Soc. 1985, 107 (16), 4679-4692.

2. Tao, Z.; Gilbert, B. B.; Denmark, S. E., J. Am. Chem. Soc. 2019, 141 (48), 19161-19170.

3. Péron, F.; Fossey, C.; Cailly, T.; Fabis, F., Org. Lett. 2012, 14 (7), 1827-1829.

4. (a) Liu, Y.; Wu, J.; Qian, B.; Shang, Y., Org. Biomol. Chem. 2019, 17 (38), 8768-8777;

(b) Borah, A. J.; Phukan, P., Chem. Commun. 2012, 48 (44), 5491-5493.

5. Péron, F.; Fossey, C.; Sopkova-de Oliveira Santos, J.; Cailly, T.; Fabis, F., Chem. Eur. J. 2014, 20 (24), 7507-7513.

6. (a) Singh, K.; Staig, S. J.; Weaver, J. D., J. Am. Chem. Soc. 2014, 136 (14), 5275-5278; (b)

Karnakanti, S.; Zang, Z.-L.; Zhao, S.; Shao, P.-L.; Hu, P.; He, Y., Chem. Commun. 2017, $53(81), 11205-11208$.

7. Jensen, T.; Pedersen, H.; Bang-Andersen, B.; Madsen, R.; Jørgensen, M., Angew. Chem. Int. Ed. 2008, 47 (5), 888-890.

8. Cummings, S. P.; Le, T.-N.; Fernandez, G. E.; Quiambao, L. G.; Stokes, B. J., J. Am. Chem. Soc. 2016, 138 (19), 6107-6110.

9. Berthiol, F.; Doucet, H.; Santelli, M., Eur. J. Org. Chem. 2005, 2005 (7), 1367-1377.

10. (a) Pérez, M.; Fañanás-Mastral, M.; Hornillos, V.; Rudolph, A.; Bos, P. H.; Harutyunyan, S. R.; Feringa, B. L., Chem. Eur. J. 2012, 18 (38), 11880-11883; (b) Han, S.; Shen, X.; Kong, D.; Zi, G.; Hou, G.; Zhang, J., J. Org. Chem. 2019, 84 (7), 4318-4329.

11. Dyker, G.; Körning, J.; Stirner, W., Eur. J. Org. Chem. 1998, 1998 (1), 149-154.

12. Daniel, P. E.; Weber, A. E.; Malcolmson, S. J., Org. Lett. 2017, 19 (13), 3490-3493.

13. Alamillo-Ferrer, C.; Curle, J. M.; Davidson, S. C.; Lucas, S. C. C.; Atkinson, S. J.; 
Campbell, M.; Kennedy, A. R.; Tomkinson, N. C. O., J. Org. Chem. 2018, 83 (12), 67286740.

14. Peng, X.-X.; Wei, D.; Han, W.-J.; Chen, F.; Yu, W.; Han, B., ACS Catal. 2017, 7 (11), 7830-7834.

15. (a) Takesue, T.; Fujita, M.; Sugimura, T.; Akutsu, H., Org. Lett. 2014, 16 (17), 4634-4637;

(b) Kathe, P. M.; Caciuleanu, A.; Berkefeld, A.; Fleischer, I., J. Org. Chem. 2020, 85 (23), 15183-15196.

16. Tummatorn, J.; Ruchirawat, S.; Ploypradith, P., Chem. Eur. J. 2010, 16 (5), 1445-1448.

17. Cornish, C. A.; Warren, S., J. Chem. Soc., Perkin Trans. 1 1985, (0), 2585-2598.

18. Rauf, W.; Brown, J. M., Angew. Chem. Int. Ed. 2008, 47 (22), 4228-4230.

19. Tanaka, T.; Hiramatsu, K.; Kobayashi, Y.; Ohno, H., Tetrahedron 2005, 61 (28), 67266742.

20. Cheung, F. K.; Hayes, A. M.; Hannedouche, J.; Yim, A. S. Y.; Wills, M., J. Org. Chem. 2005, 70 (8), 3188-3197.

21. (a) Yuste, F.; Ortiz, B.; Carrasco, A.; Peralta, M.; Quintero, L.; Sánchez-Obregón, R.; Walls, F.; García Ruano, J. L., Tetrahedron: Asymmetry 2000, 11 (15), 3079-3090; (b) Prelog, V.; Mutak, S., Helv. Chim. Acta 1983, 66 (7), 2274-2278. 
2i ${ }^{1} \mathrm{H}$ NMR (500 MHz, $\left.\mathrm{CDCl}_{3}\right)$

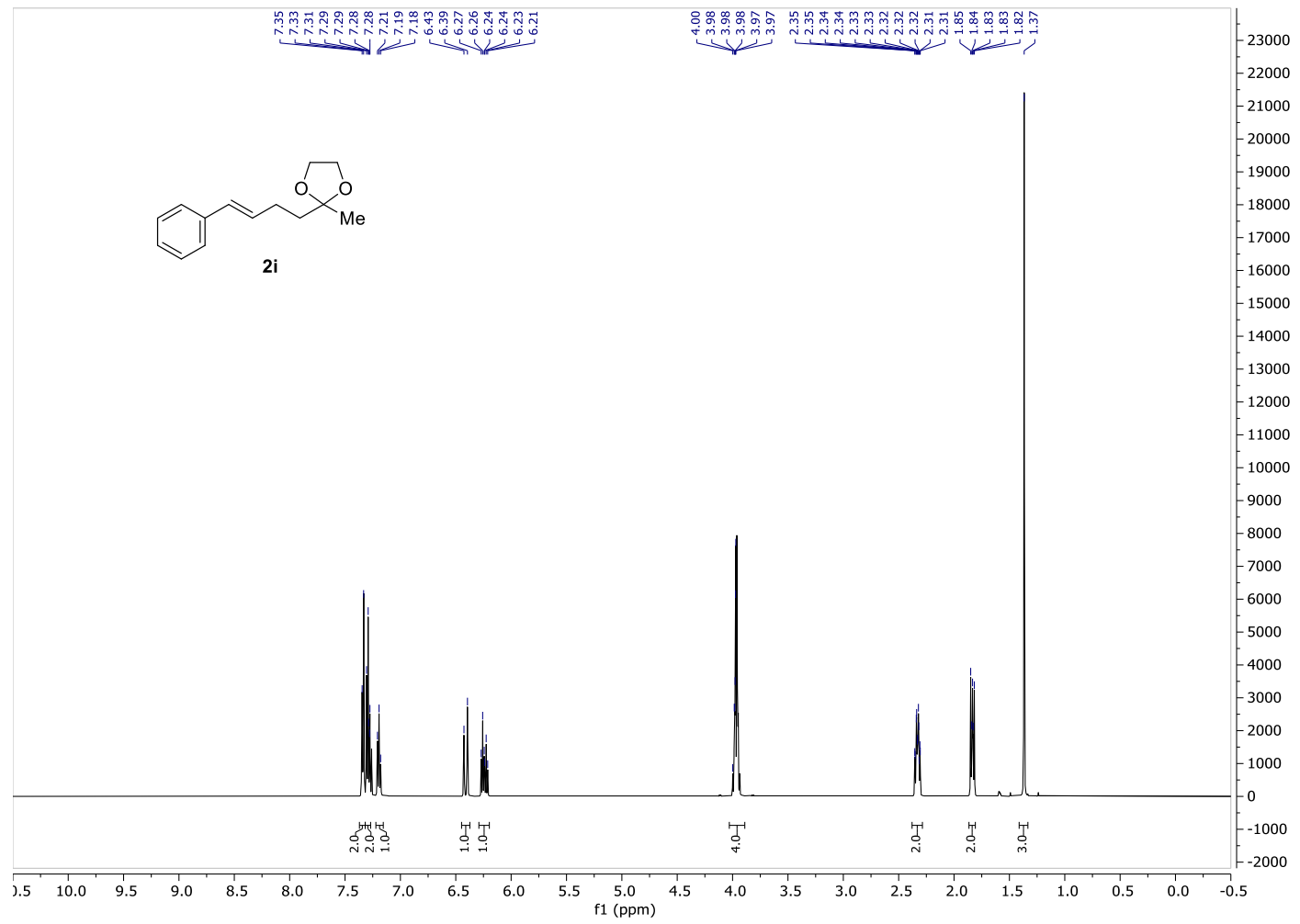

2i ${ }^{13} \mathrm{C}$ NMR (126 MHz, $\mathrm{CDCl}_{3}$ )

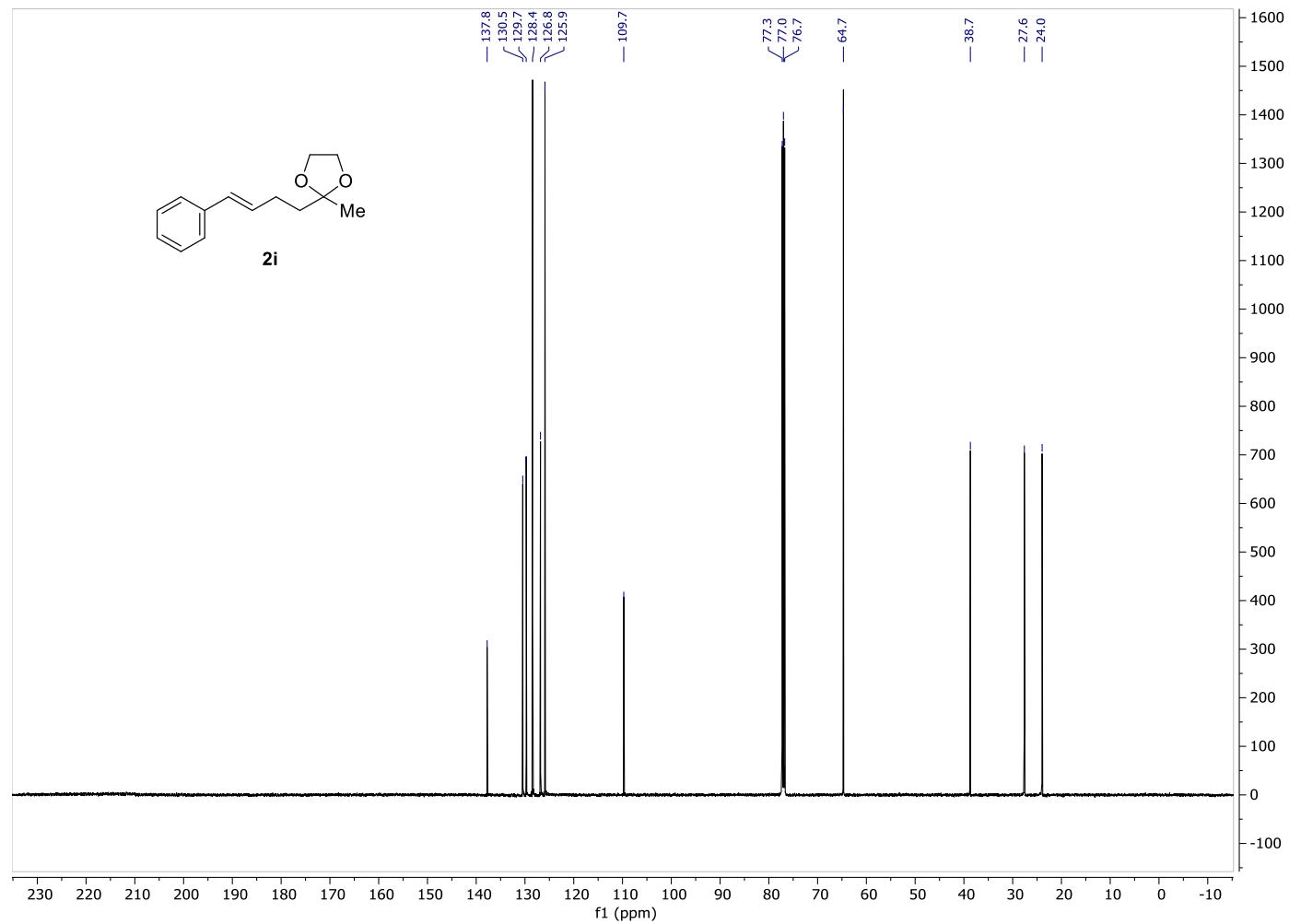


4a ${ }^{1} \mathrm{H}$ NMR (500 MHz, $\left.\mathrm{CDCl}_{3}\right)$

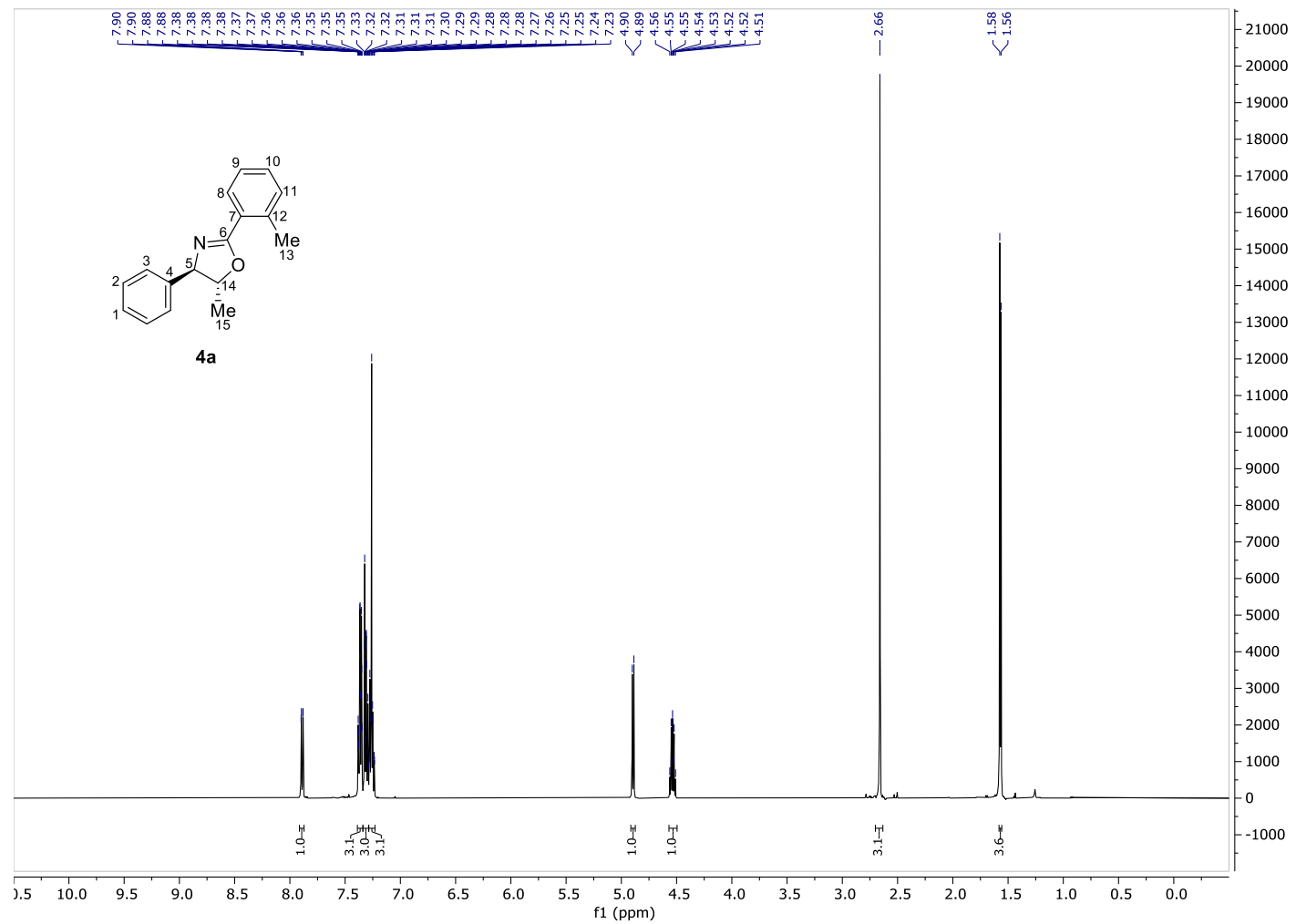

4a ${ }^{13} \mathrm{C}$ NMR (126 MHz, $\left.\mathrm{CDCl}_{3}\right)$

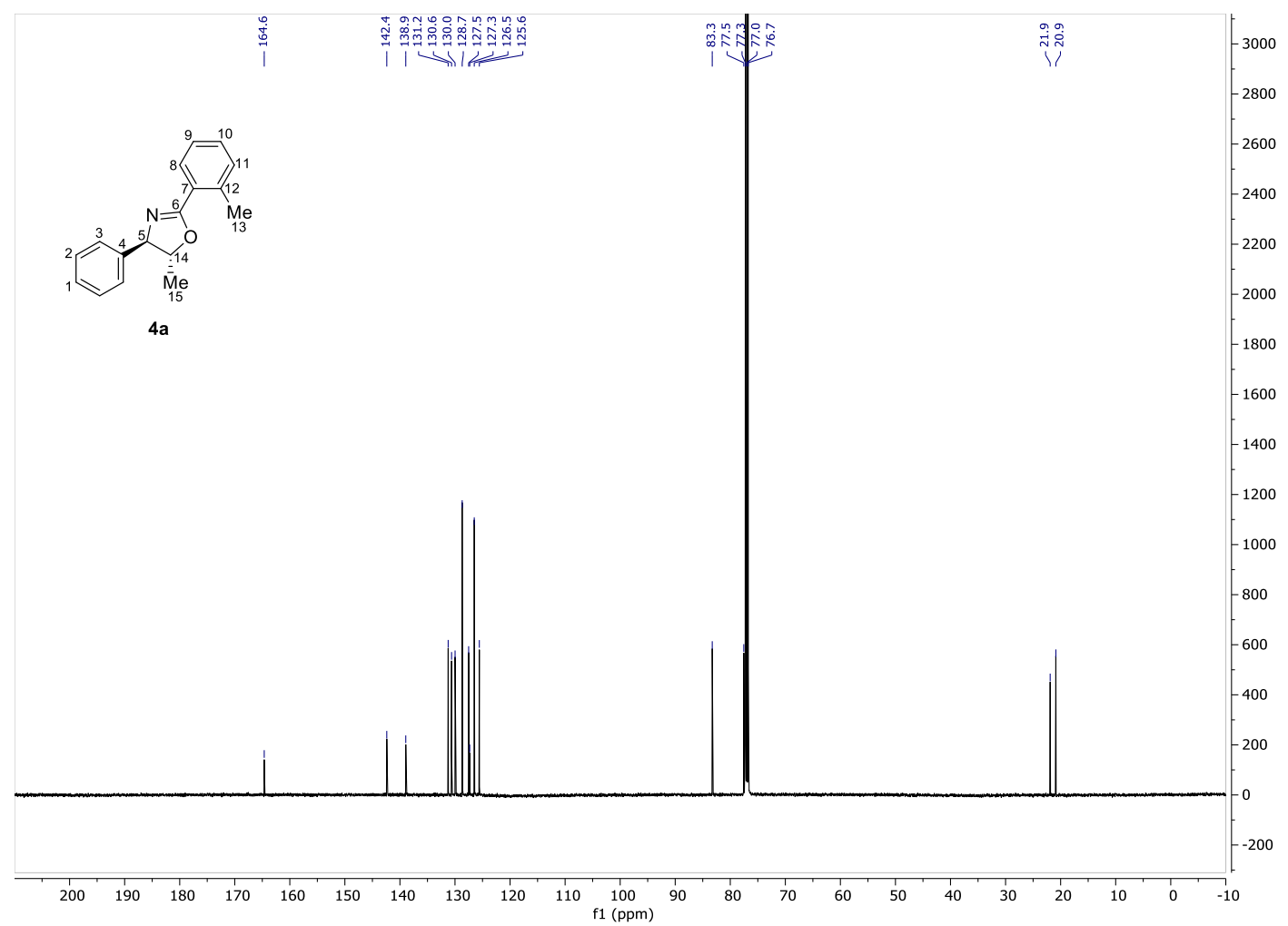




\section{4a-racemic}

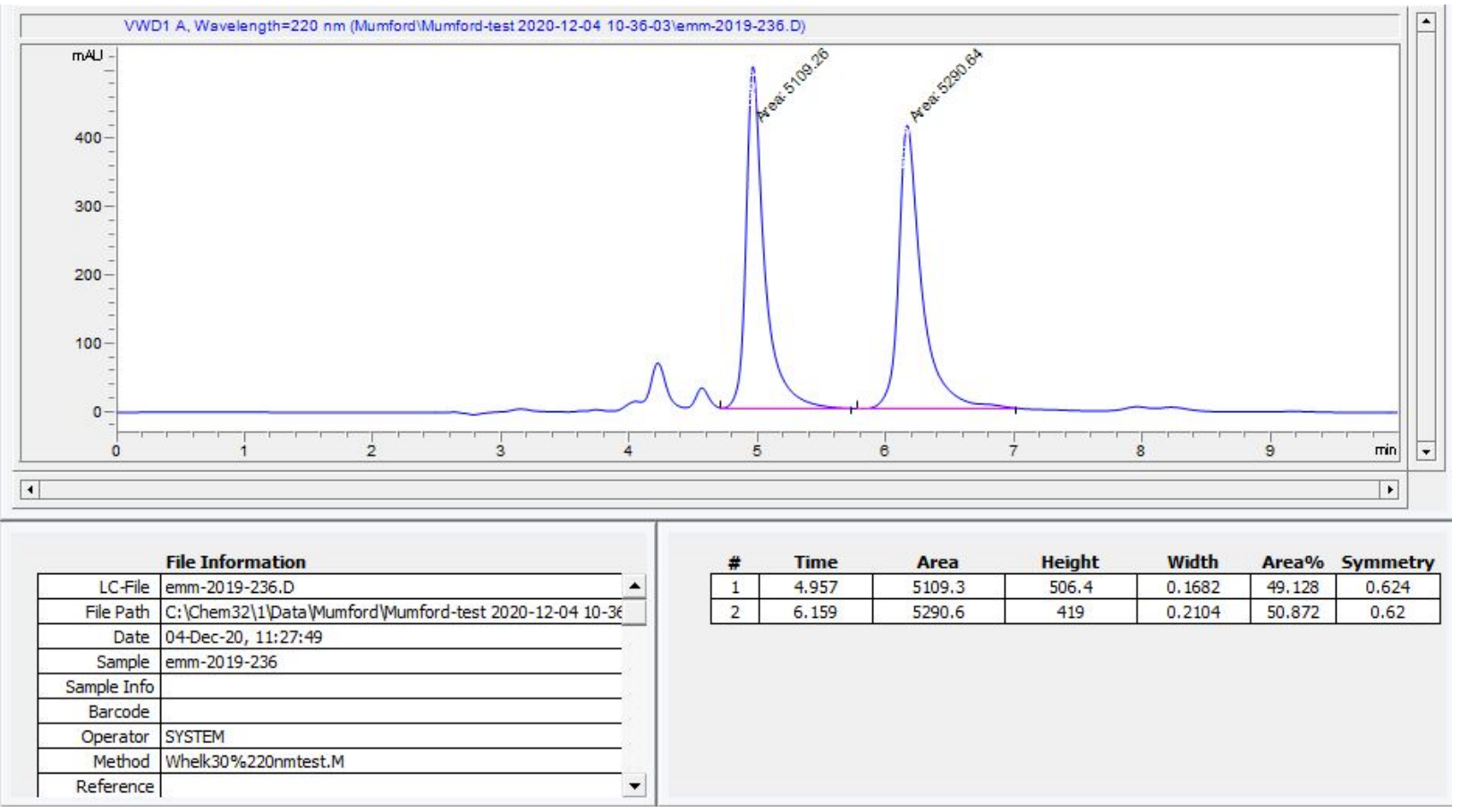

4a- $(R, R)$

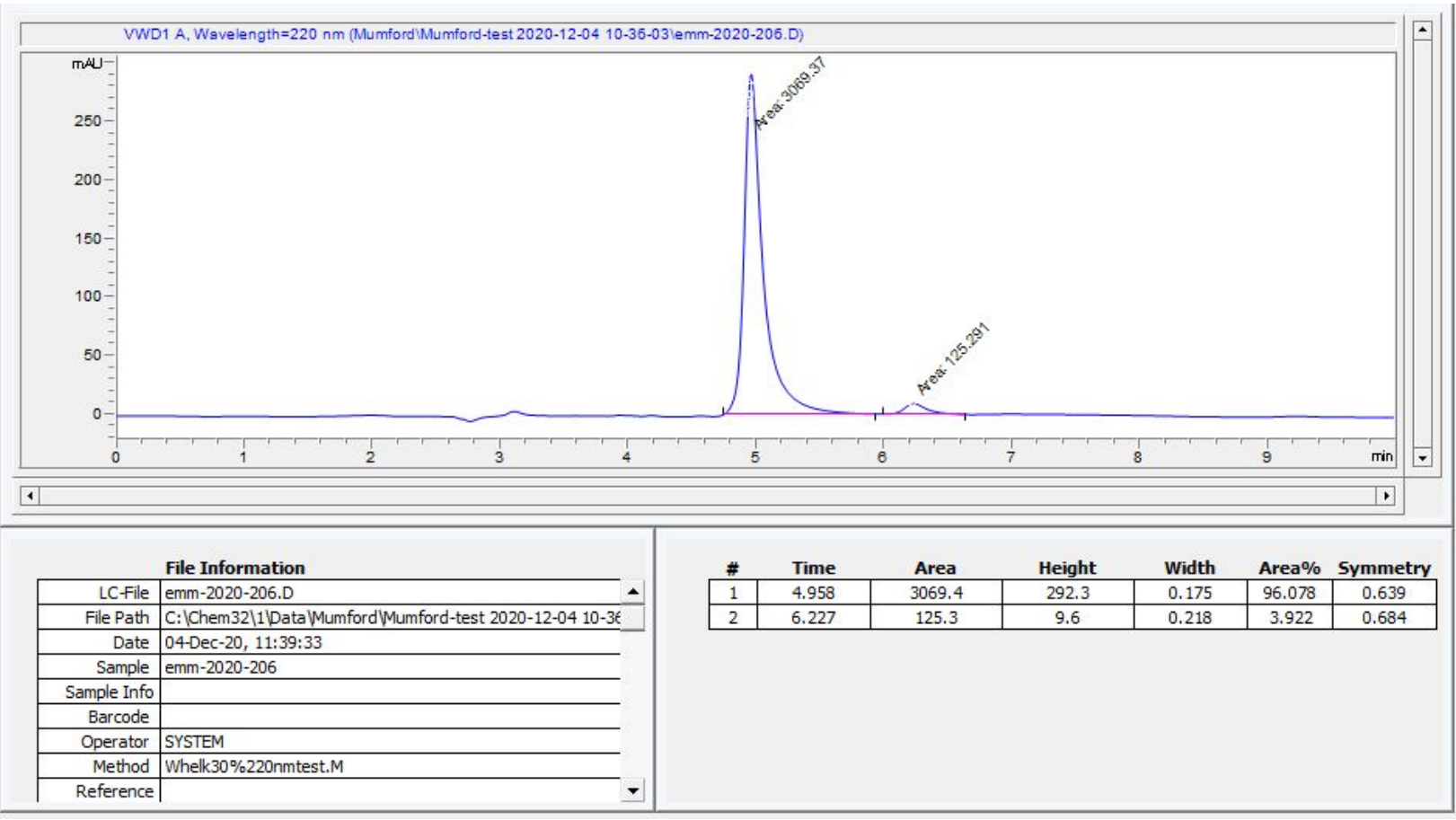


4b ${ }^{1} \mathrm{H}$ NMR (500 MHz, $\mathrm{CDCl}_{3}$ )

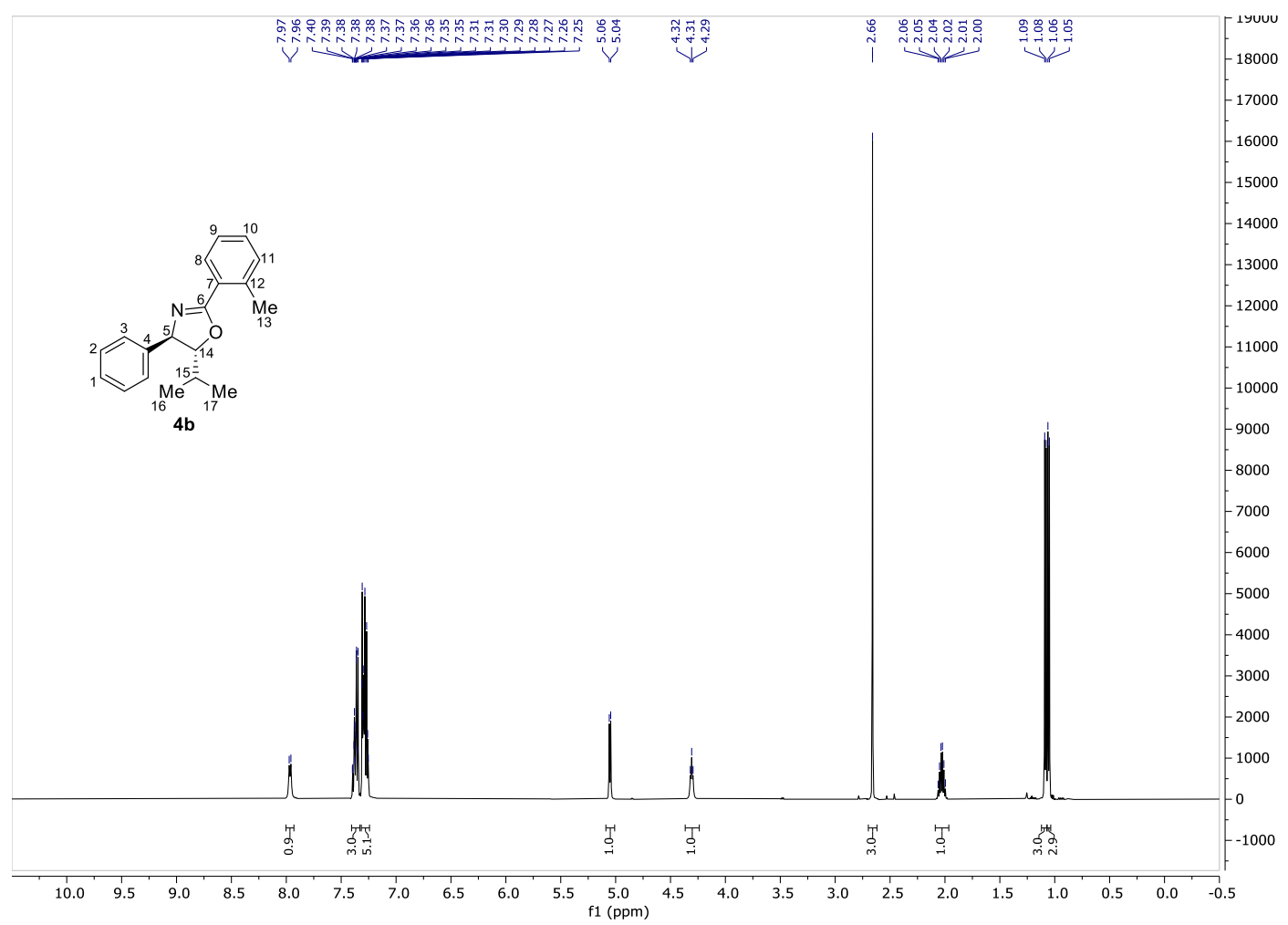

4b ${ }^{13} \mathrm{C}$ NMR (126 MHz, $\left.\mathrm{CDCl}_{3}\right)$

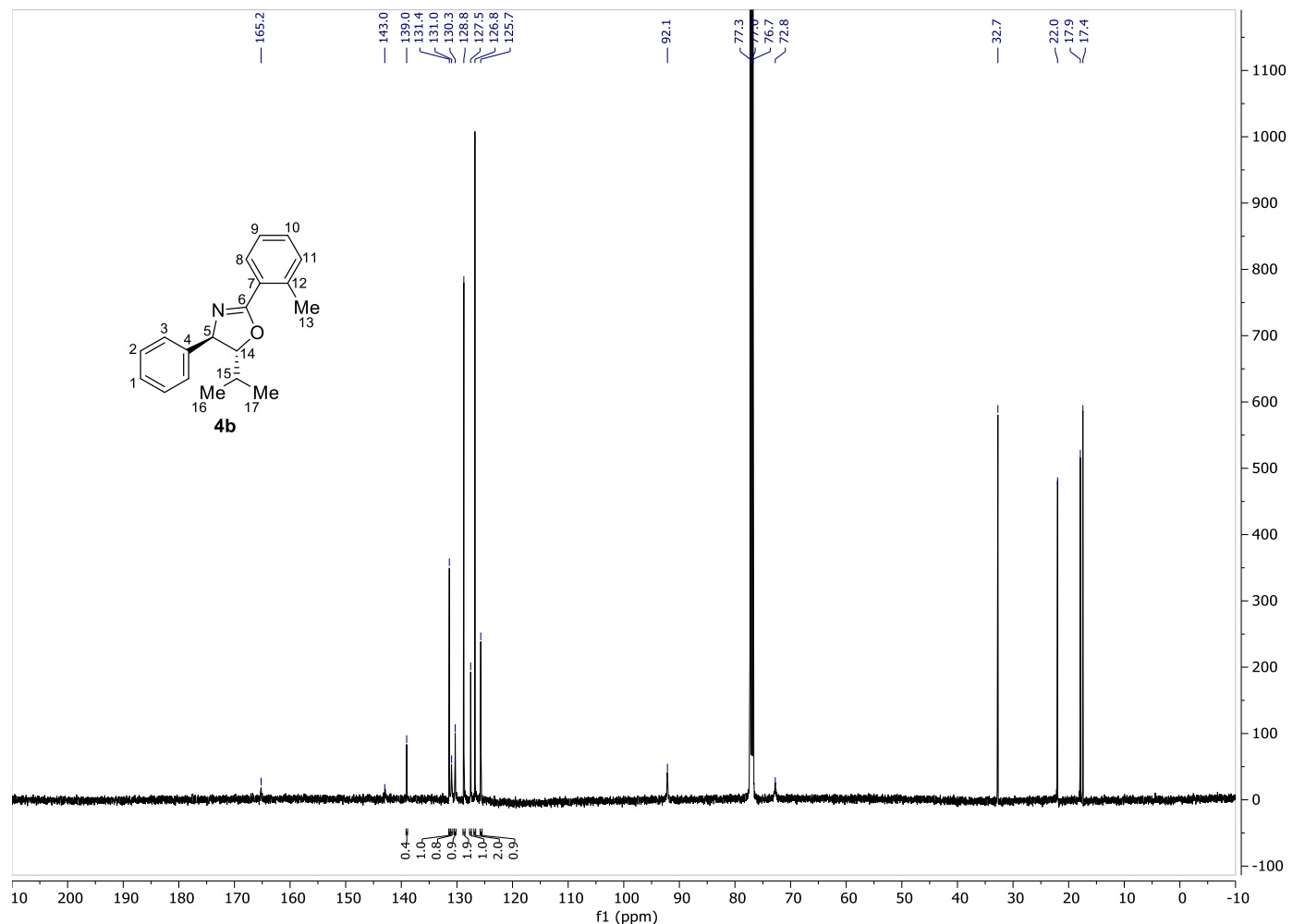




\section{4b-racemic}

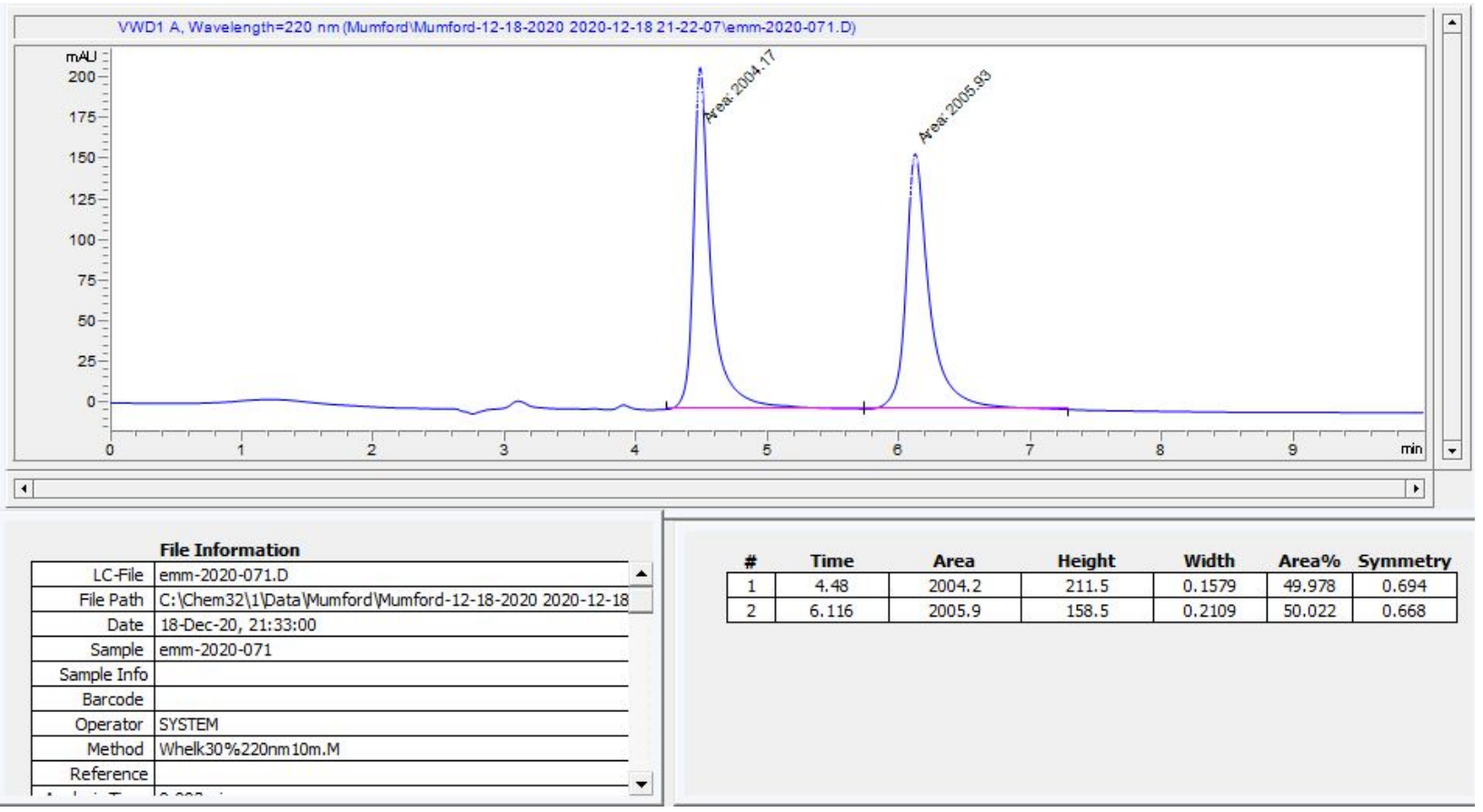

\section{4b- $(R, R)$}

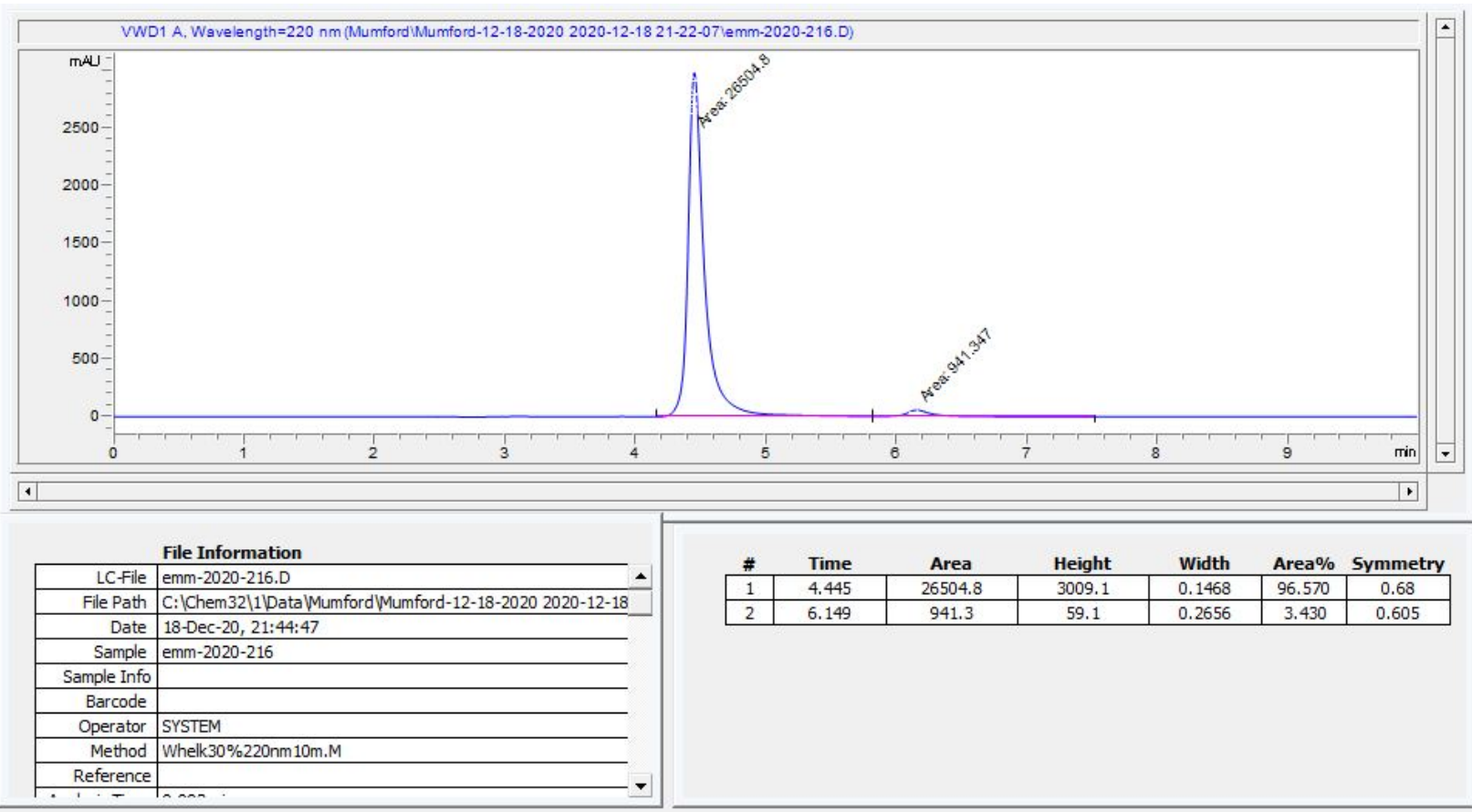




\section{4c ${ }^{1} \mathrm{H}$ NMR $\left(500 \mathrm{MHz}, \mathrm{CDCl}_{3}\right)$}

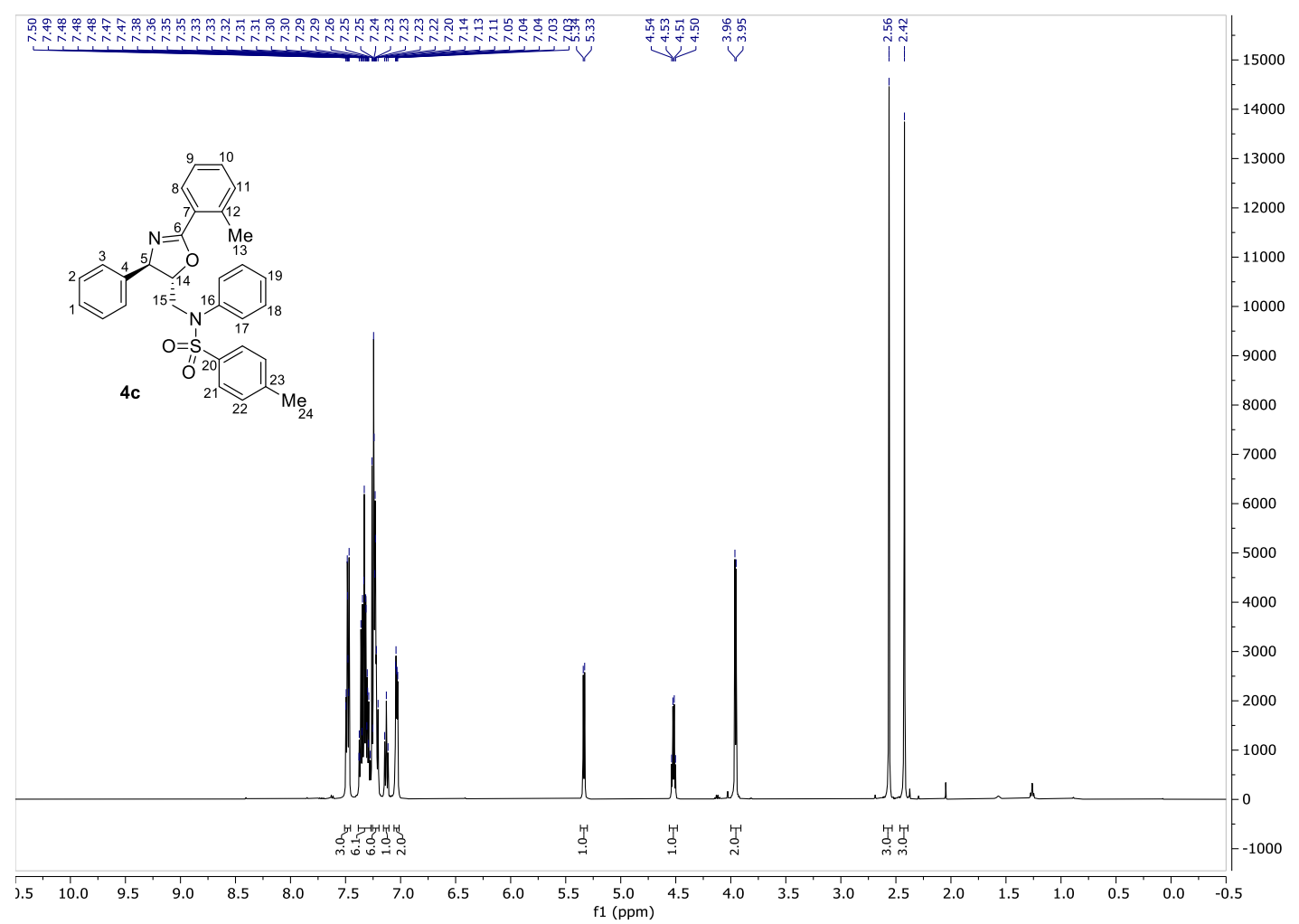

4c ${ }^{13} \mathrm{C}$ NMR (126 MHz, $\left.\mathrm{CDCl}_{3}\right)$

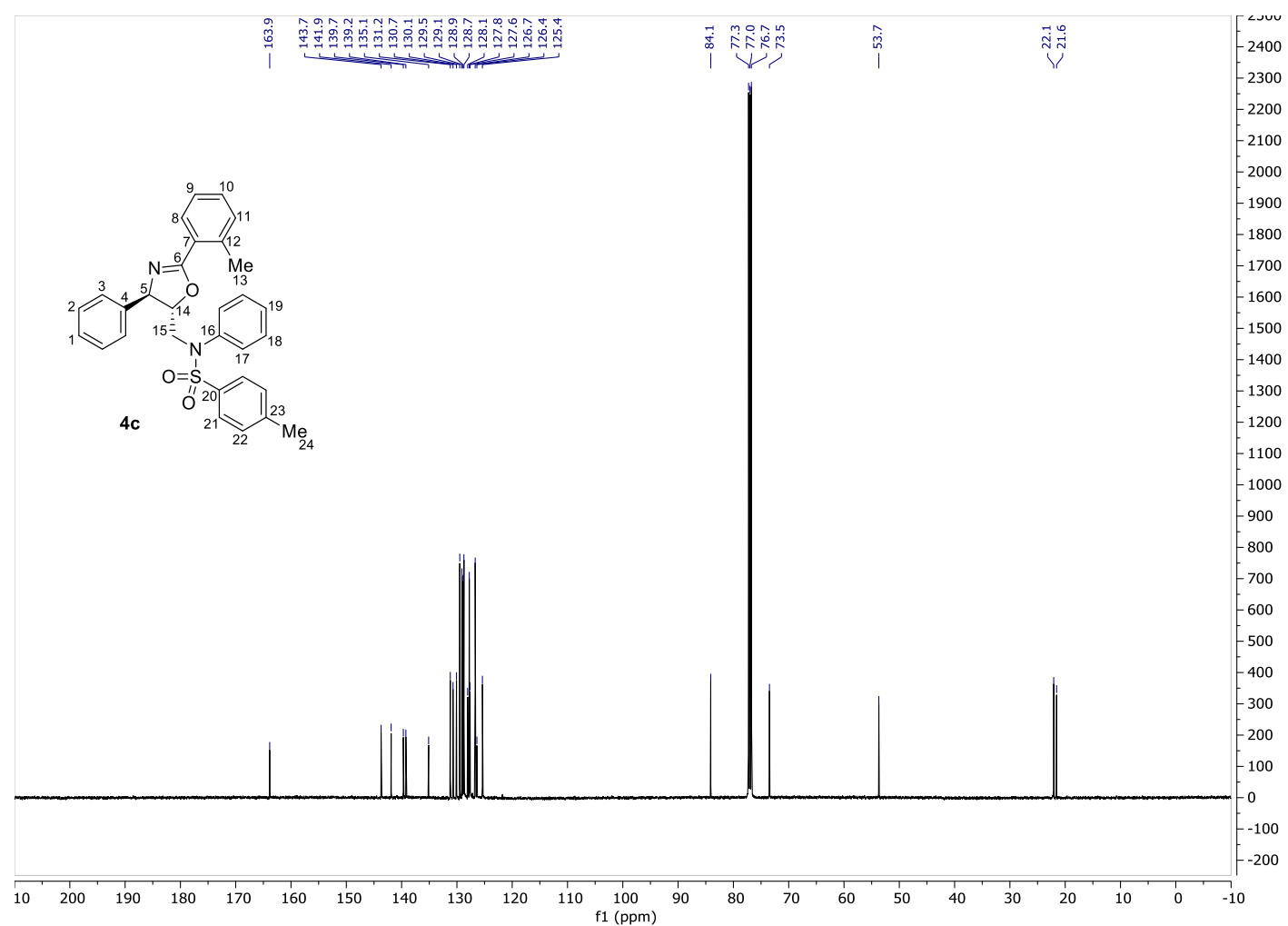




\section{4c-racemic}

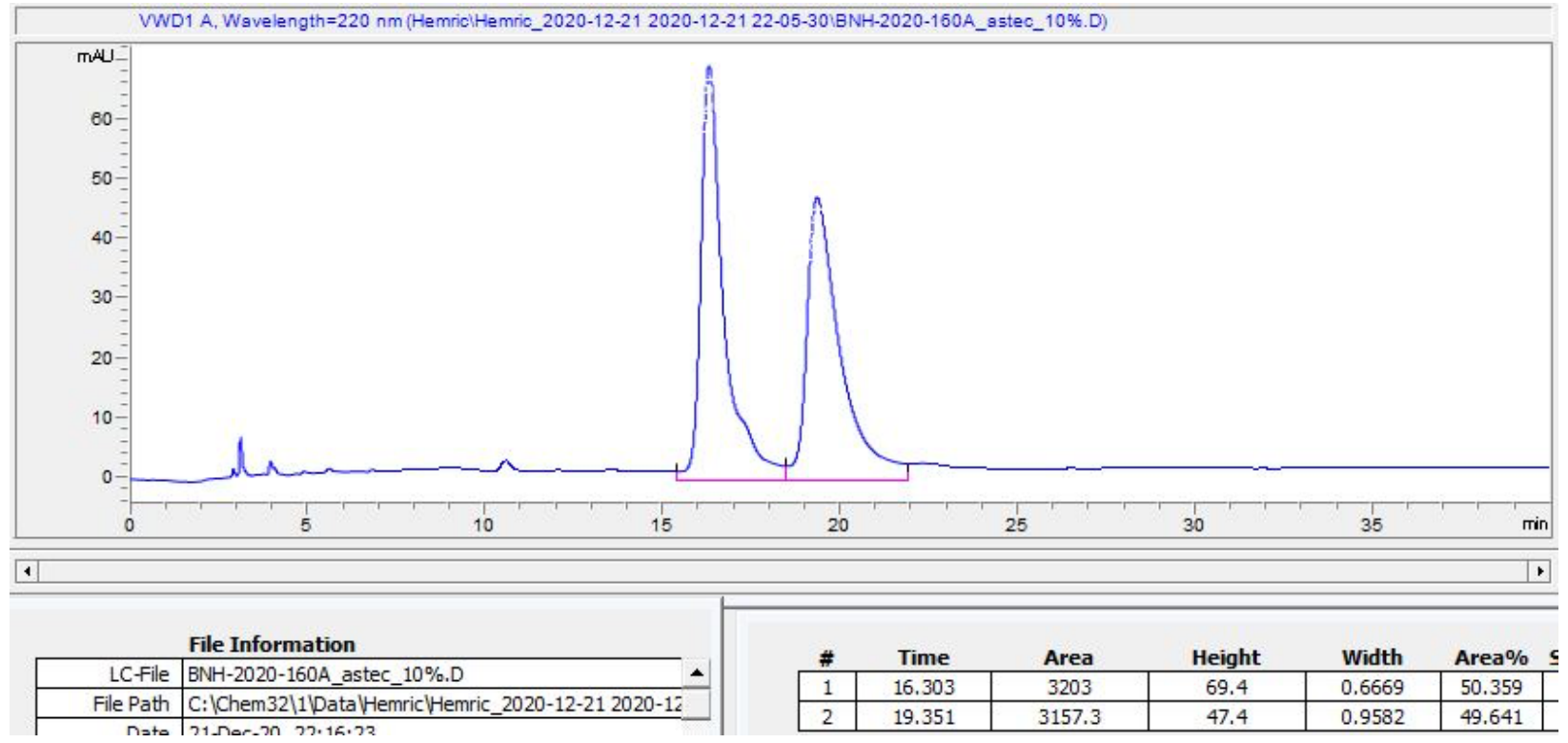

\section{$\mathbf{4 c}-(R, R)$}

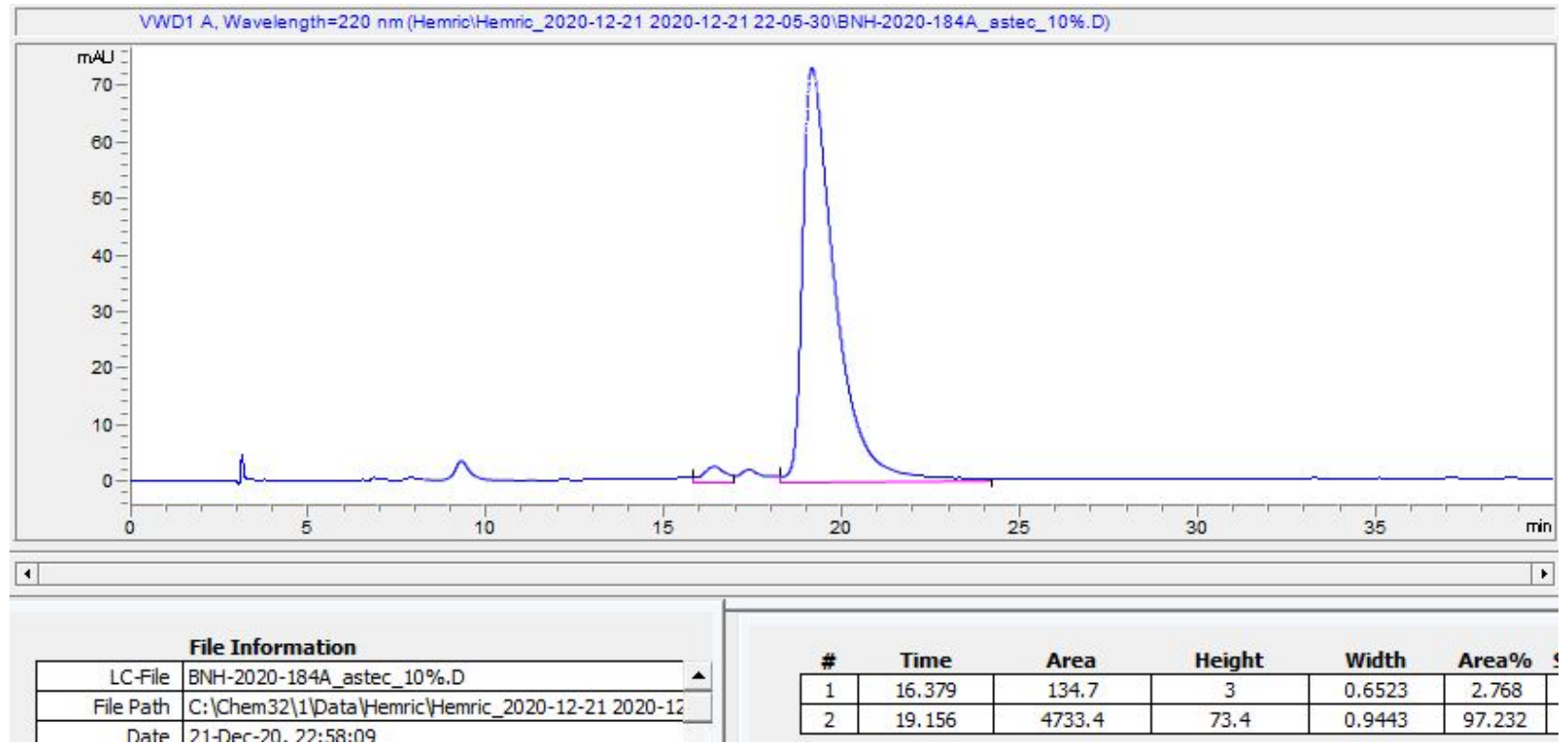


4d ${ }^{1} \mathrm{H}$ NMR (500 MHz, $\mathrm{CDCl}_{3}$ )

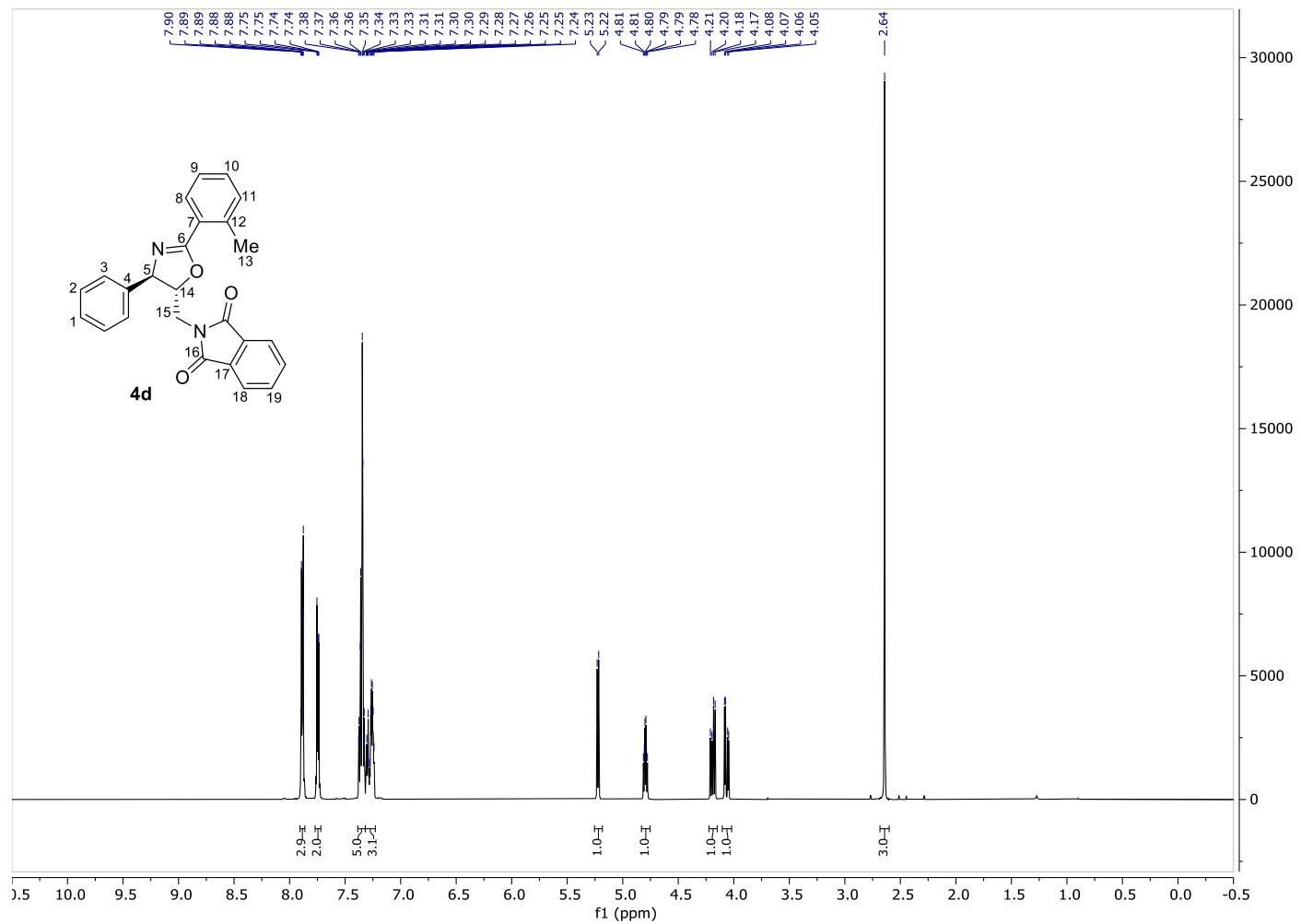

4d ${ }^{13} \mathrm{C}$ NMR (126 MHz, $\mathrm{CDCl}_{3}$ )

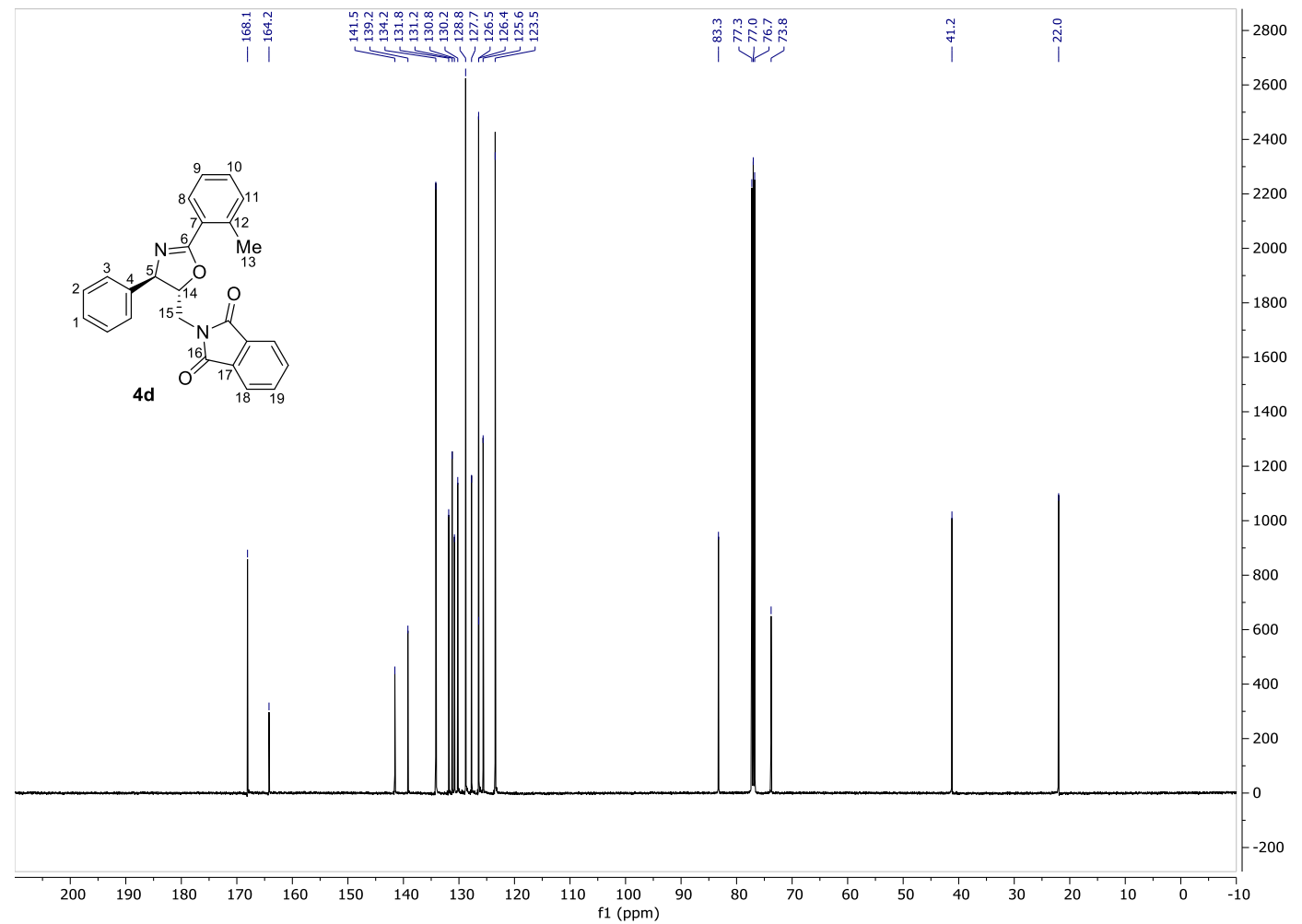




\section{4d-racemic}

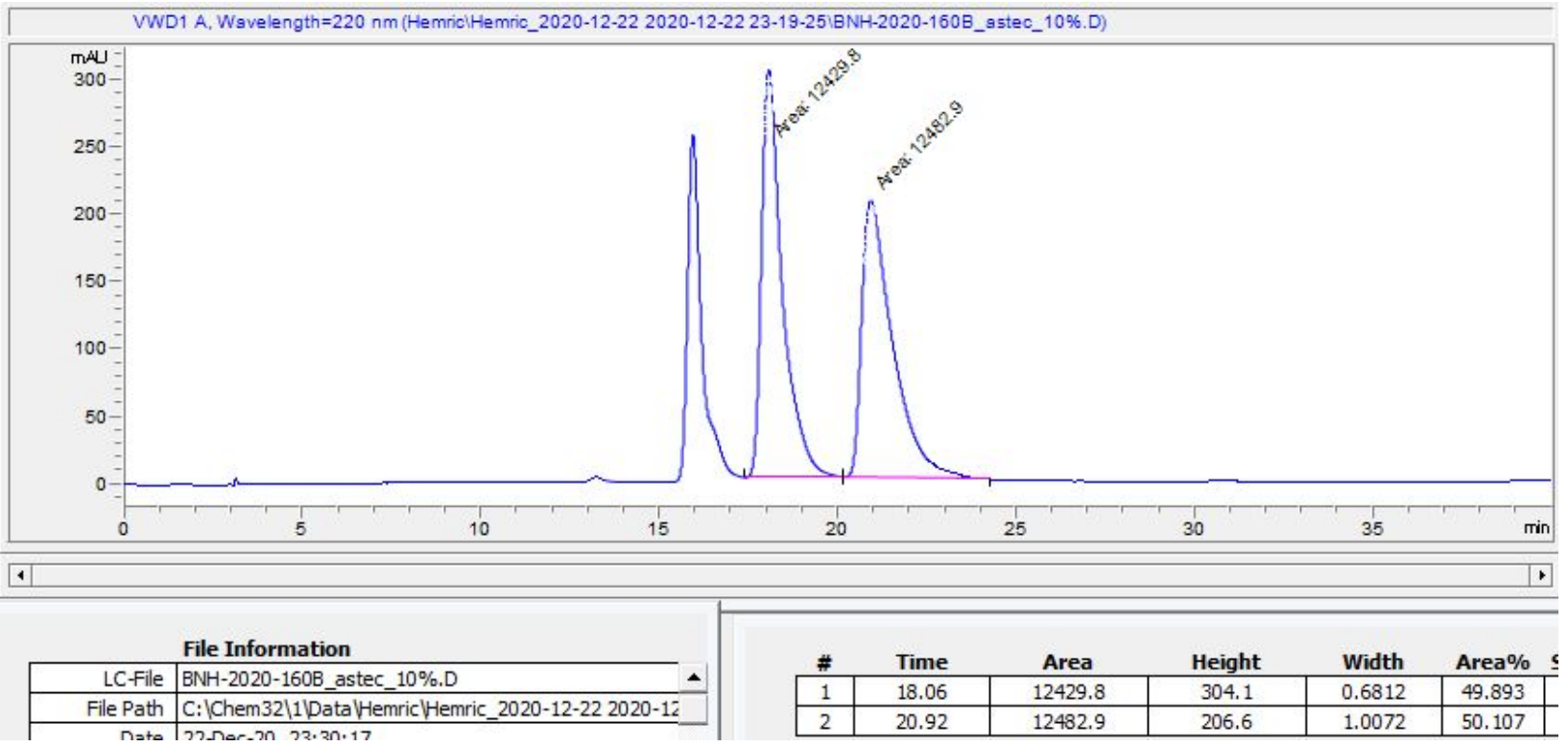

\section{4d- $(R, R)$}

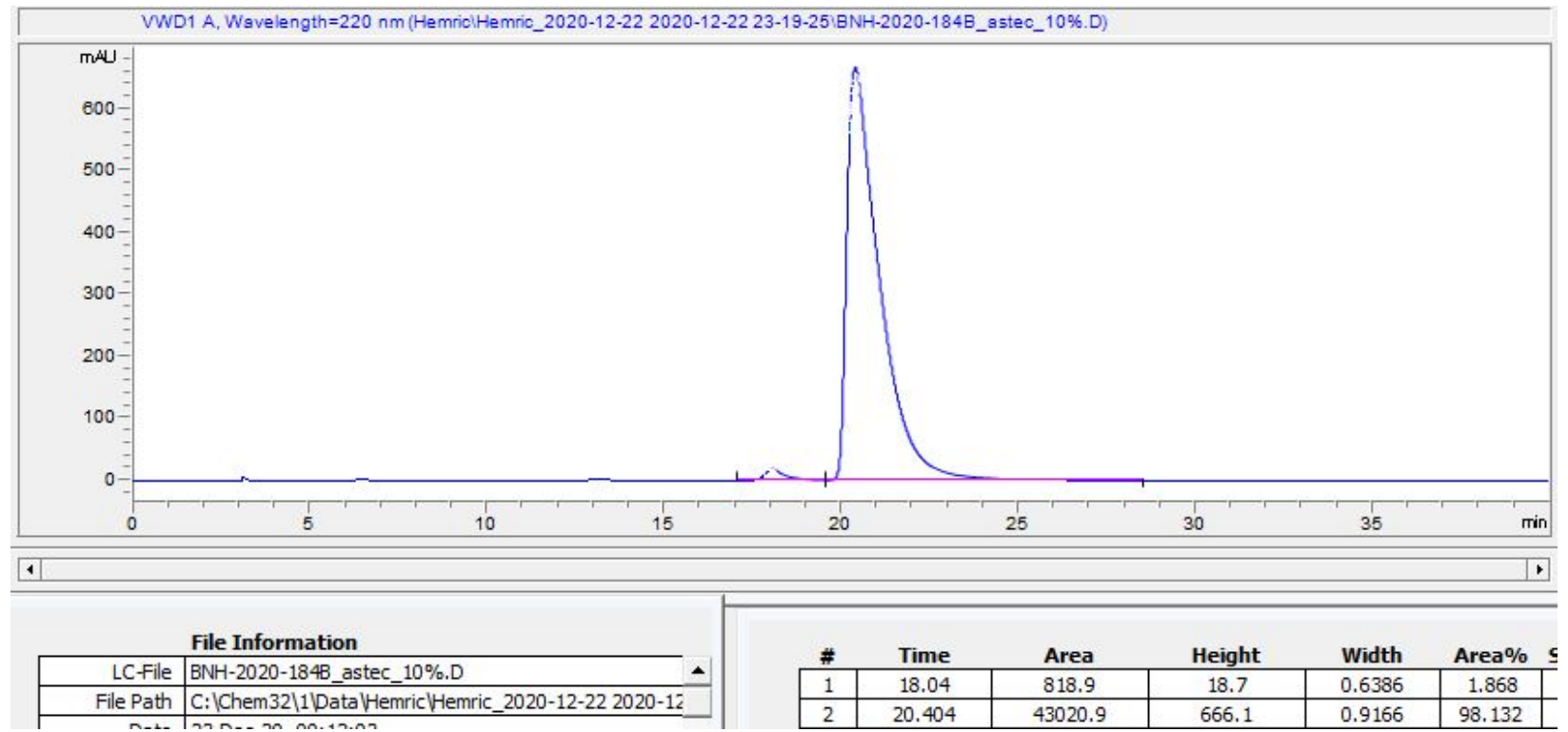


4e ${ }^{1} \mathrm{H}$ NMR (500 MHz, $\left.\mathrm{CDCl}_{3}\right)$

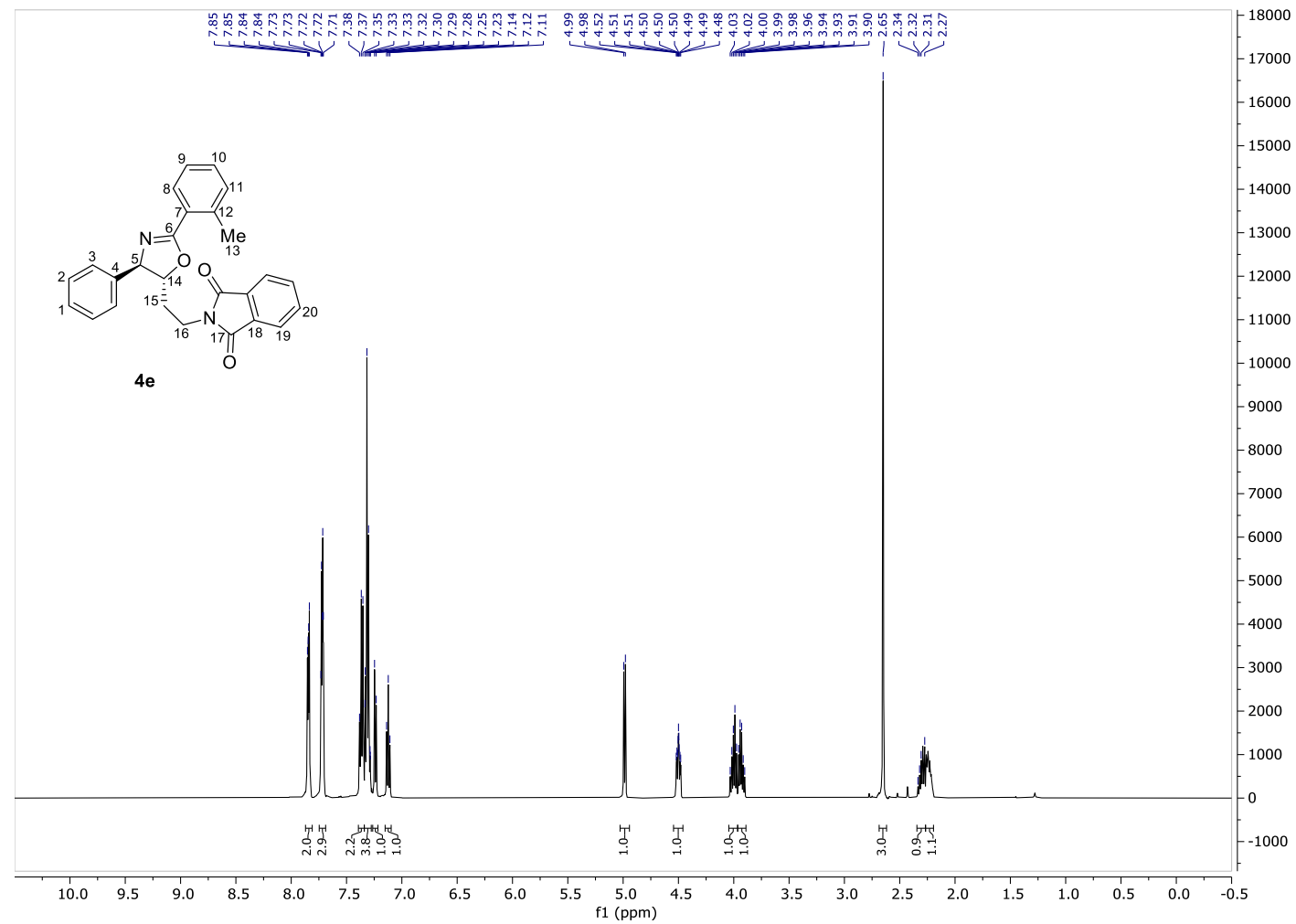

4e ${ }^{13} \mathrm{C}$ NMR (126 MHz, $\mathrm{CDCl}_{3}$ )

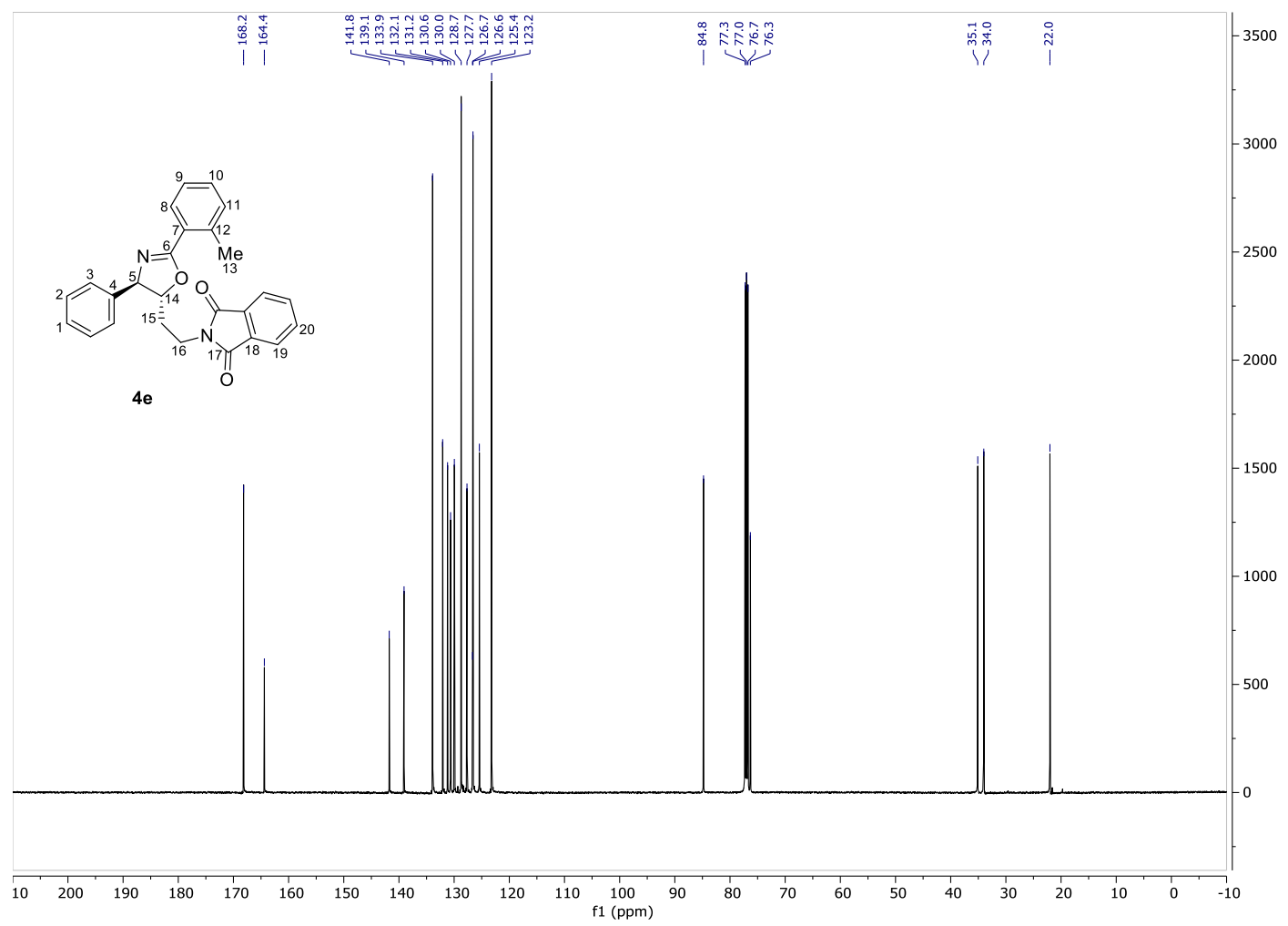




\section{4e-racemic}

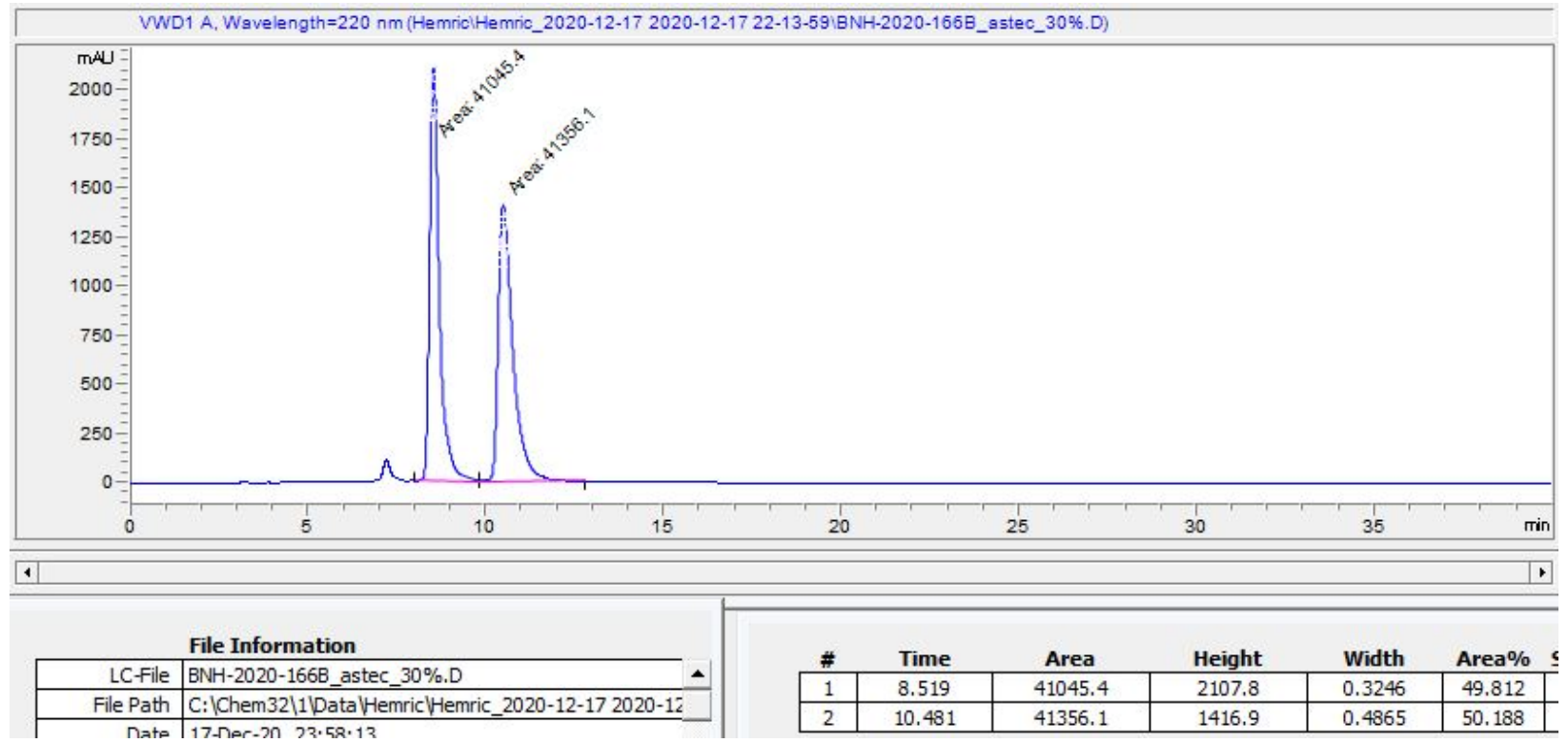

\section{$4 \mathbf{e}-(R, R)$}

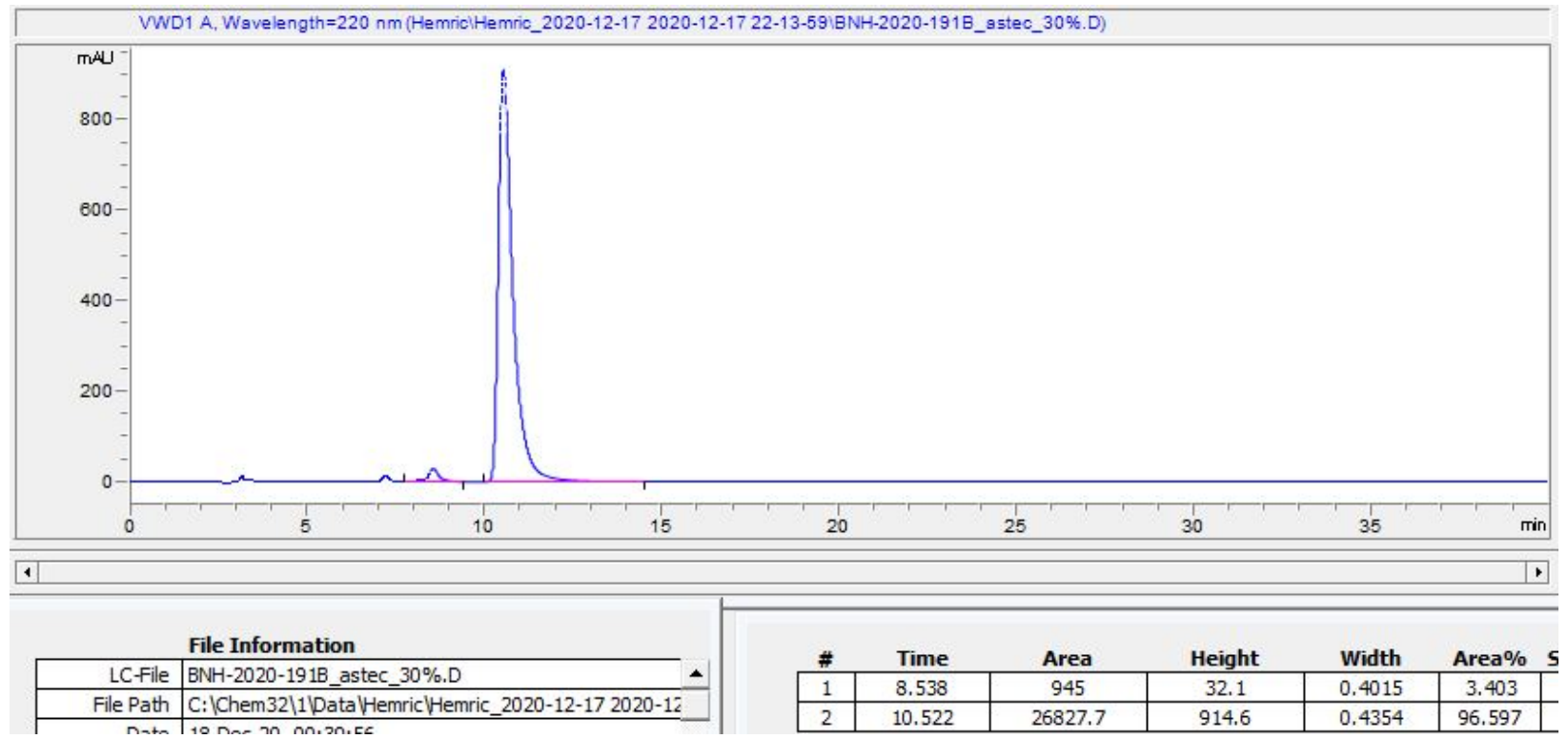




\section{4f ${ }^{1} \mathrm{H}$ NMR (500 MHz, $\left.\mathrm{CDCl}_{3}\right)$}

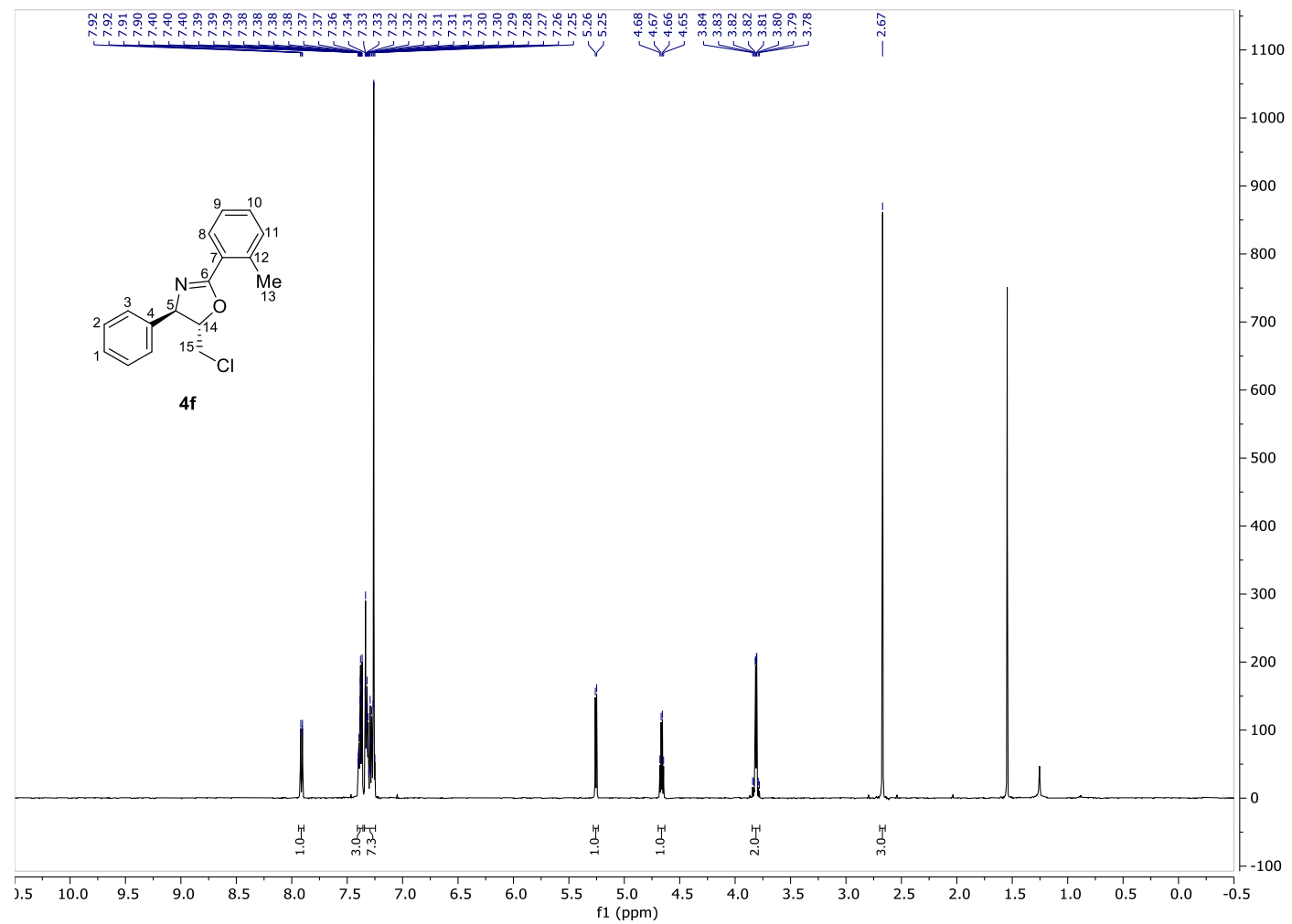

\section{4f ${ }^{13} \mathrm{C} \mathrm{NMR}\left(126 \mathrm{MHz}, \mathrm{CDCl}_{3}\right)$}

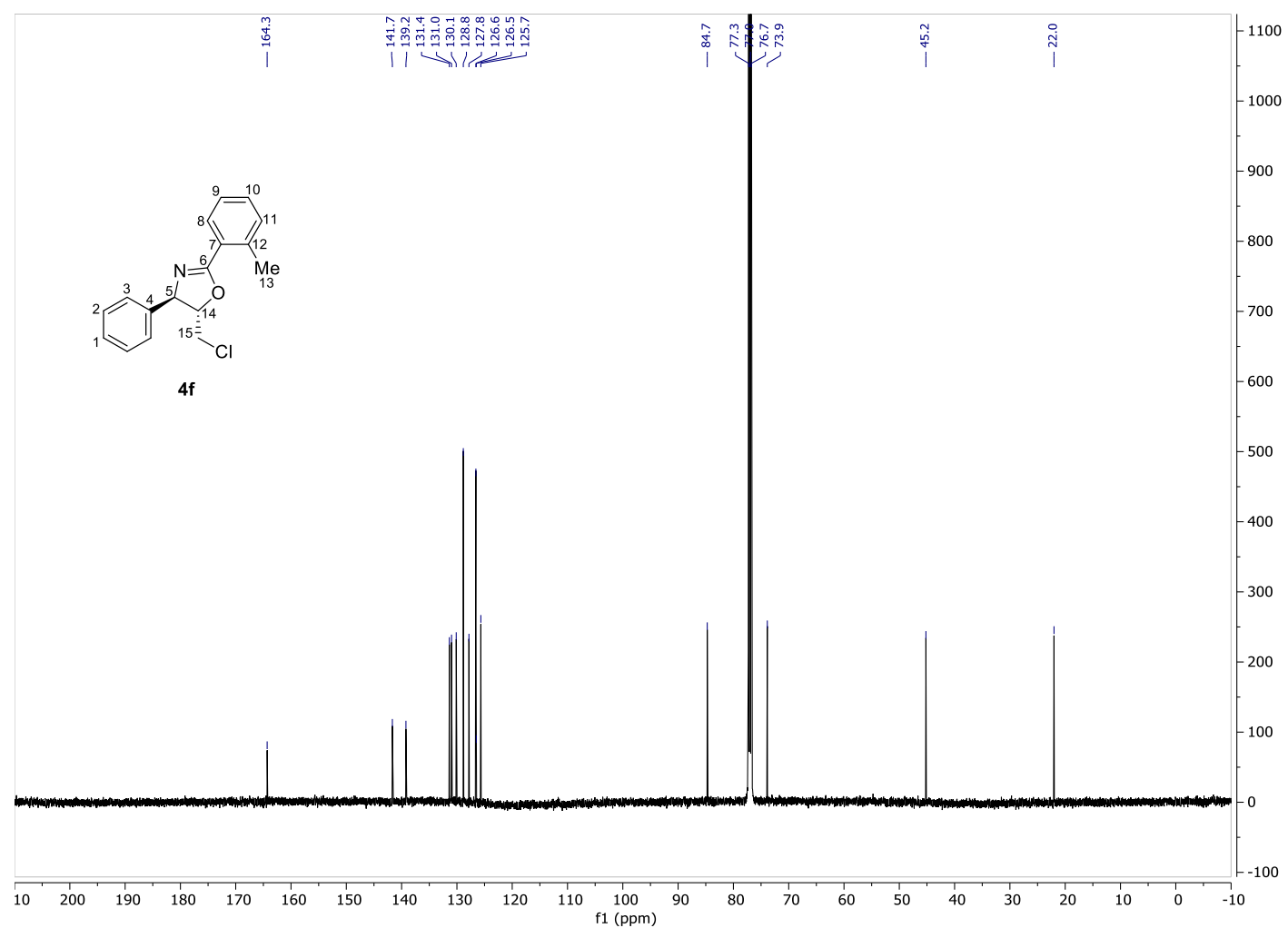




\section{4f-racemic}

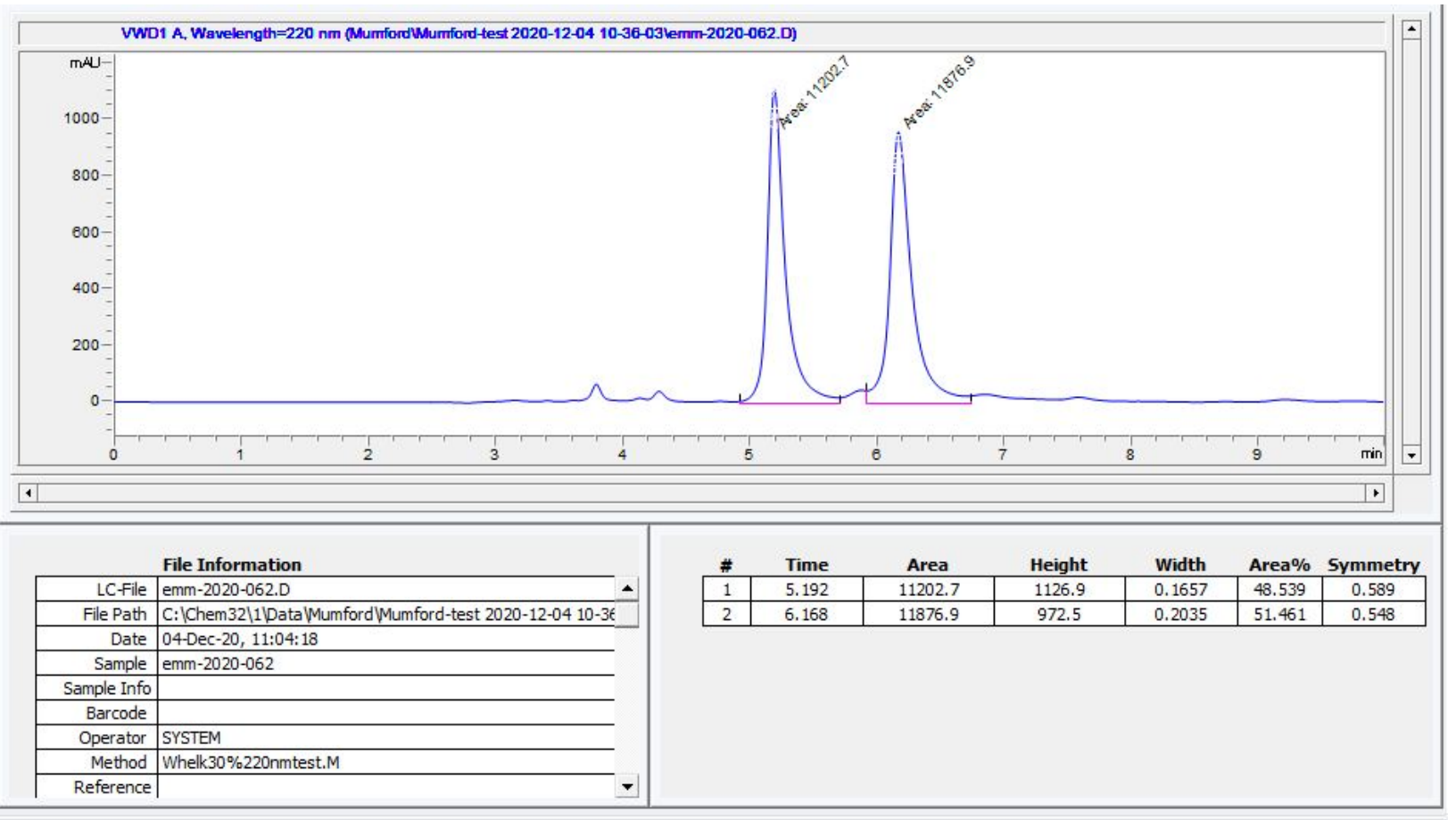

\section{4f- $(R, S)$}

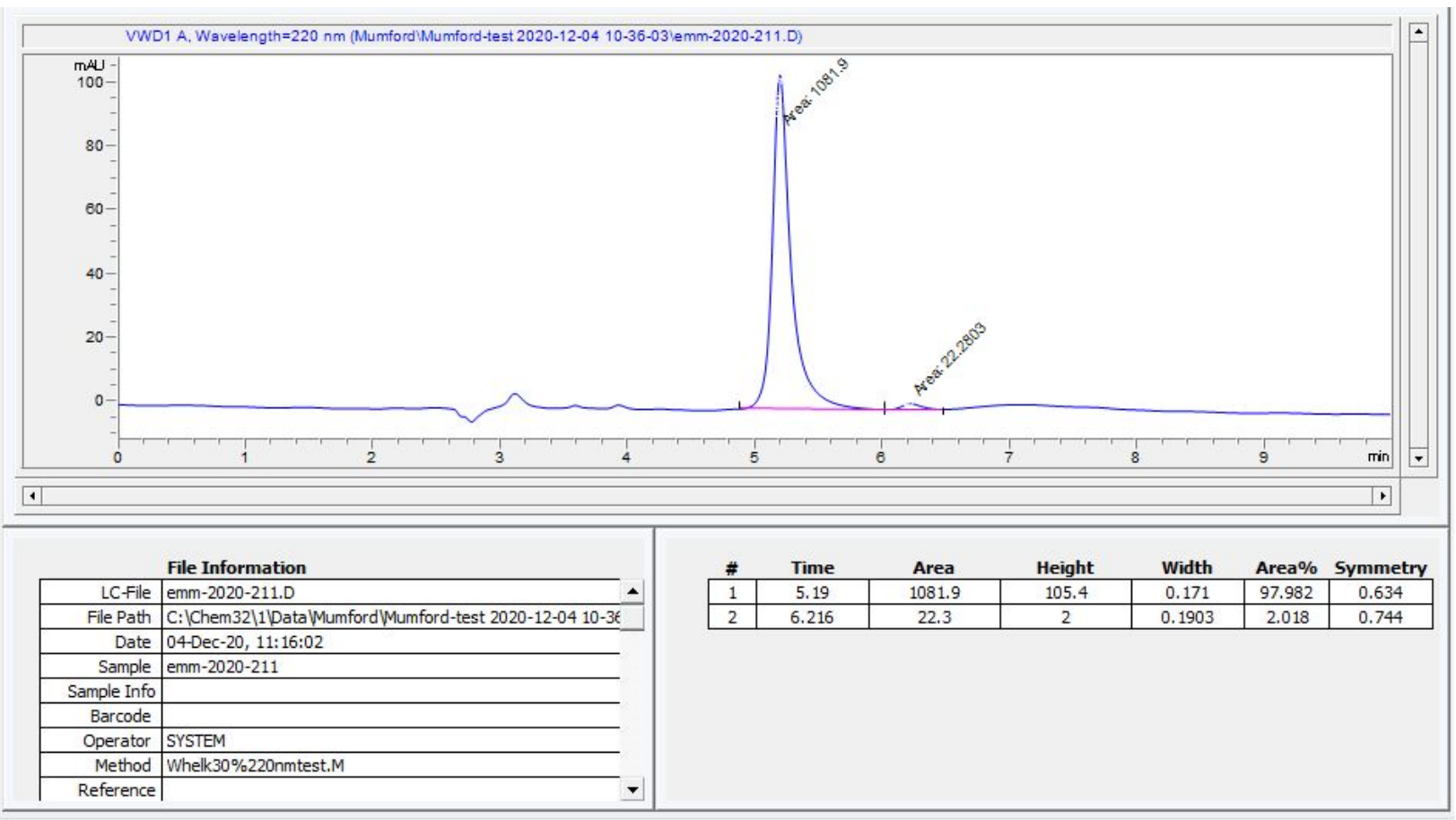


4g ${ }^{1} \mathrm{H}$ NMR (500 MHz, $\mathrm{CDCl}_{3}$ )

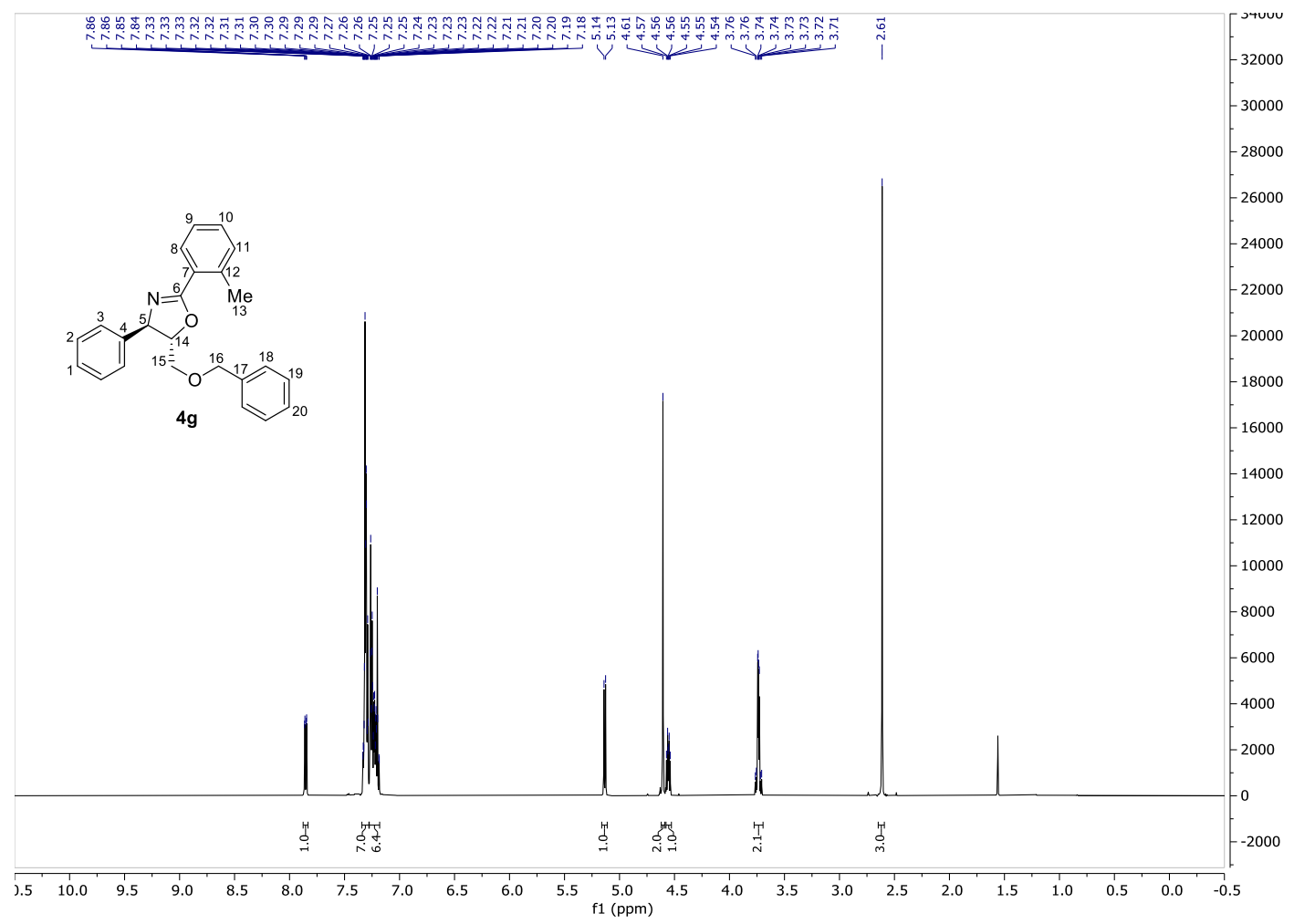

4g ${ }^{13} \mathrm{C}$ NMR (126 MHz, $\left.\mathrm{CDCl}_{3}\right)$

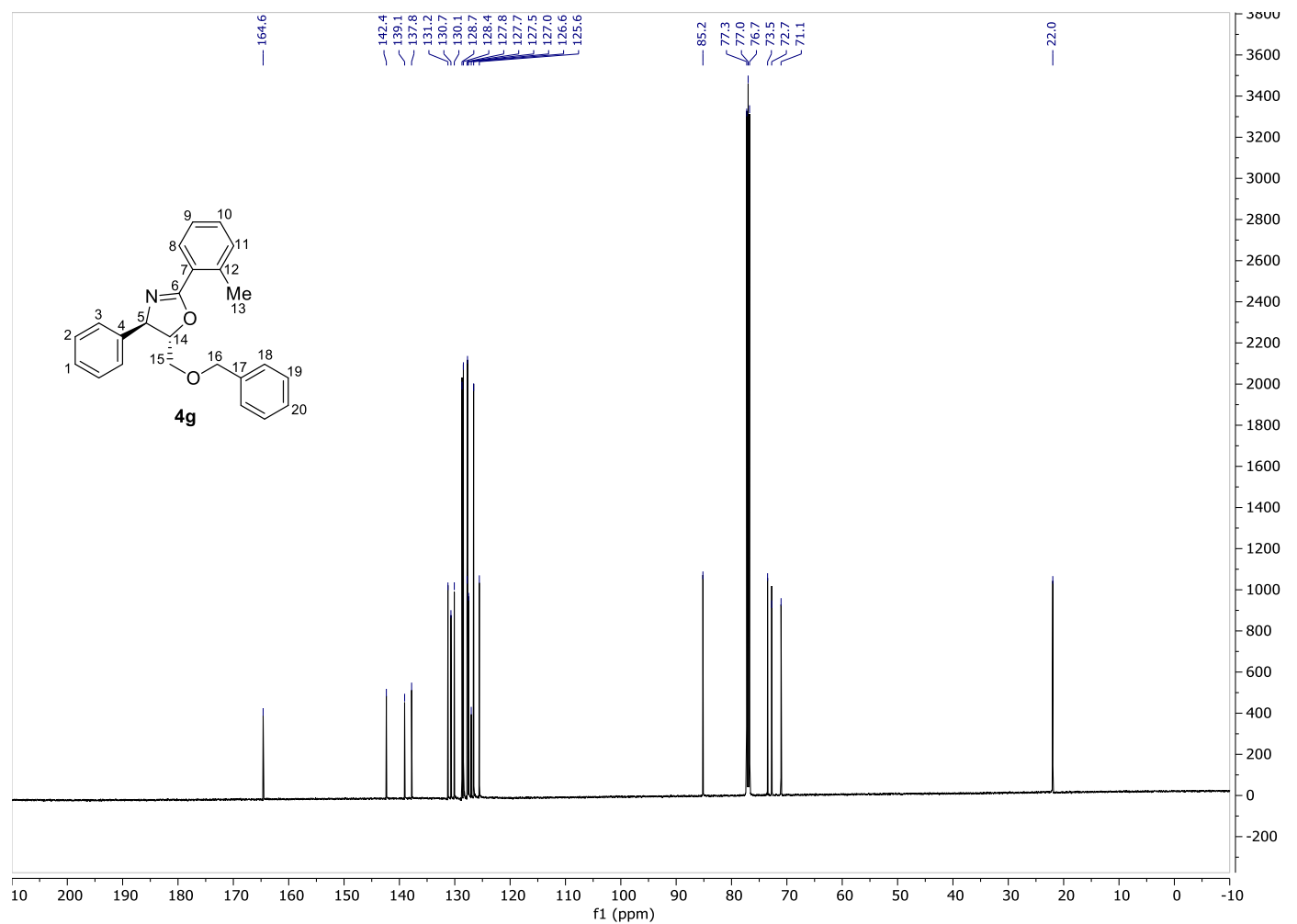


4g-racemic

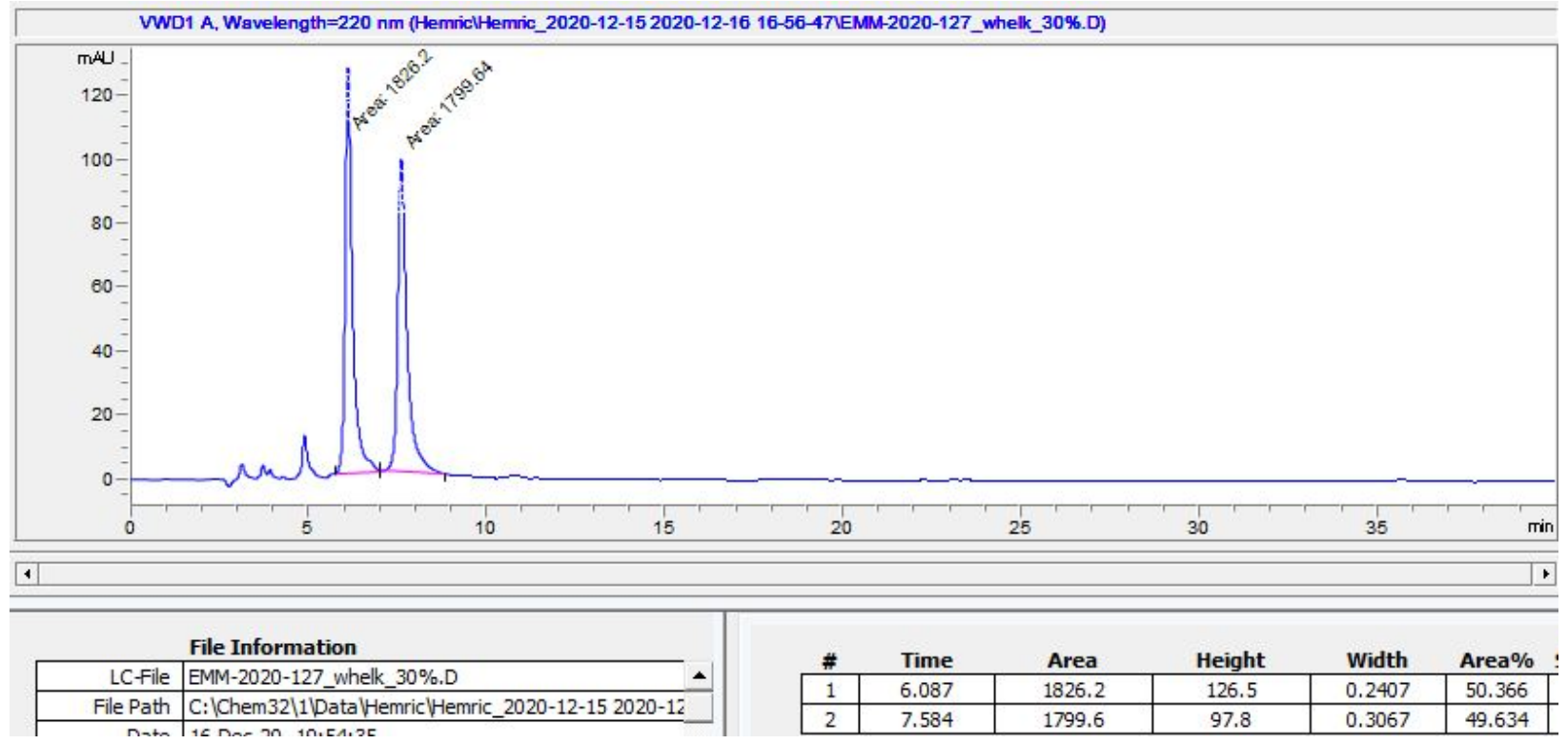

4g- $(R, S)$

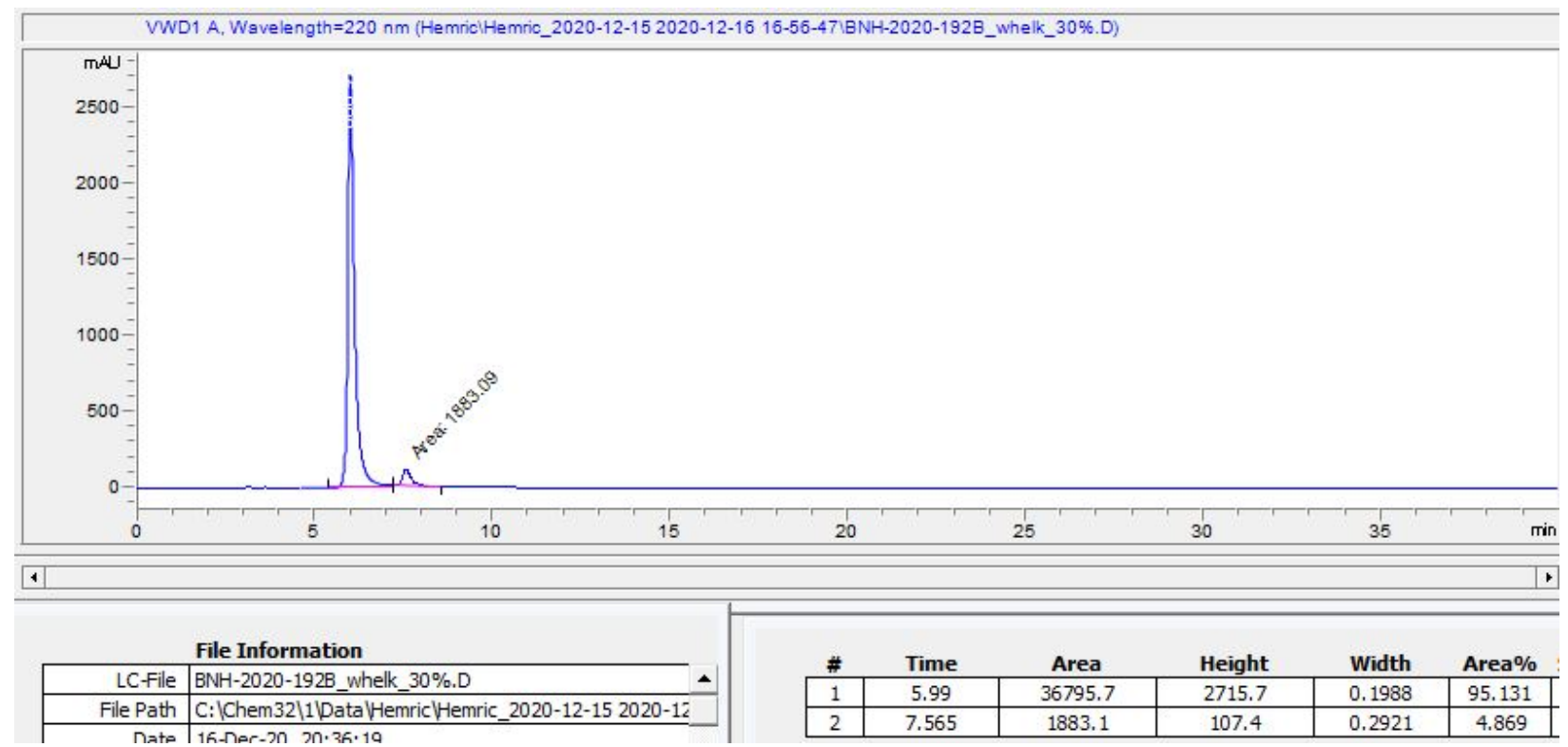


4h ${ }^{1} \mathrm{H}$ NMR (500 MHz, $\mathrm{CDCl}_{3}$ )

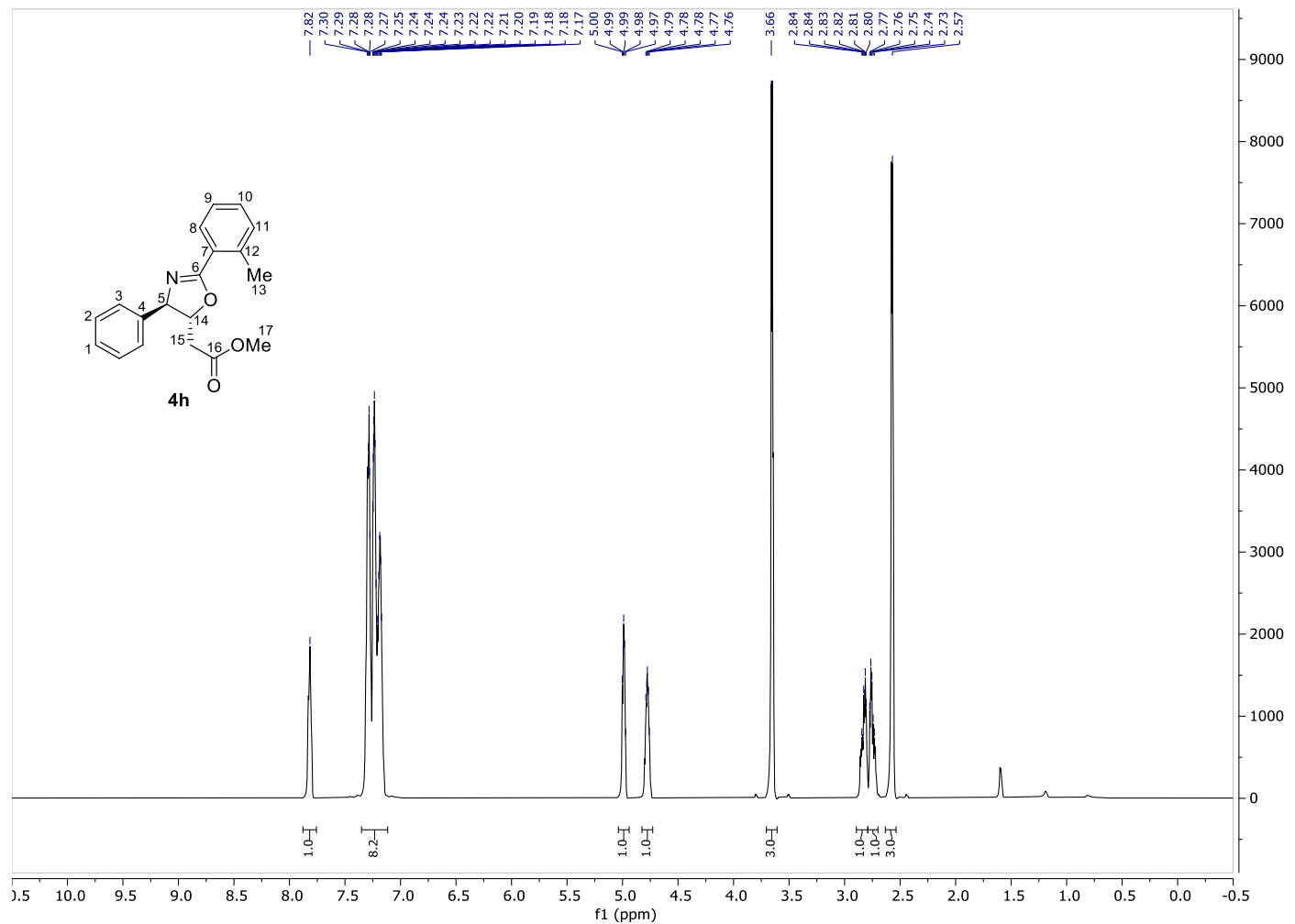

4h ${ }^{13} \mathrm{C}$ NMR (126 MHz, $\left.\mathrm{CDCl}_{3}\right)$

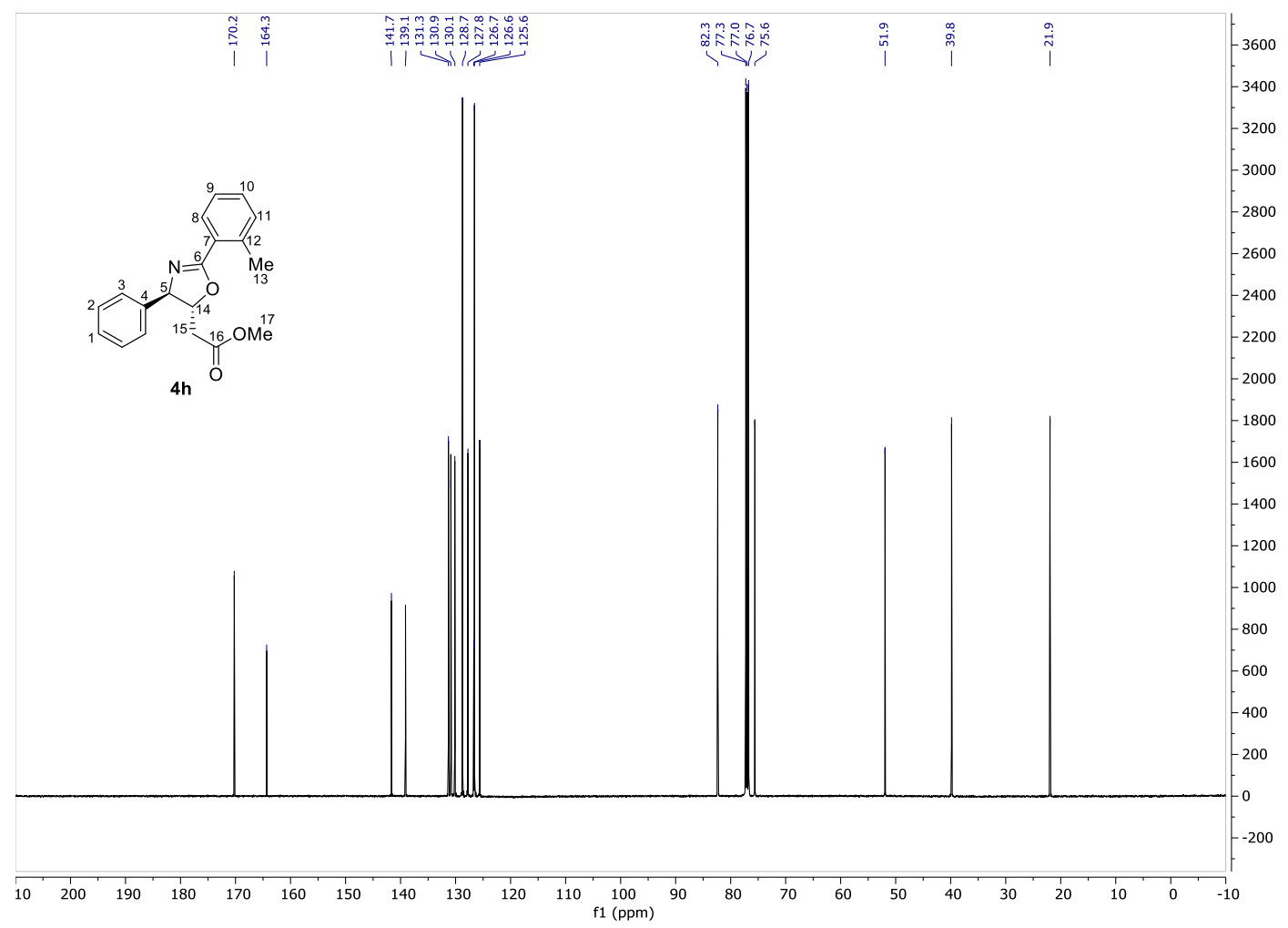




\section{4h-racemic}

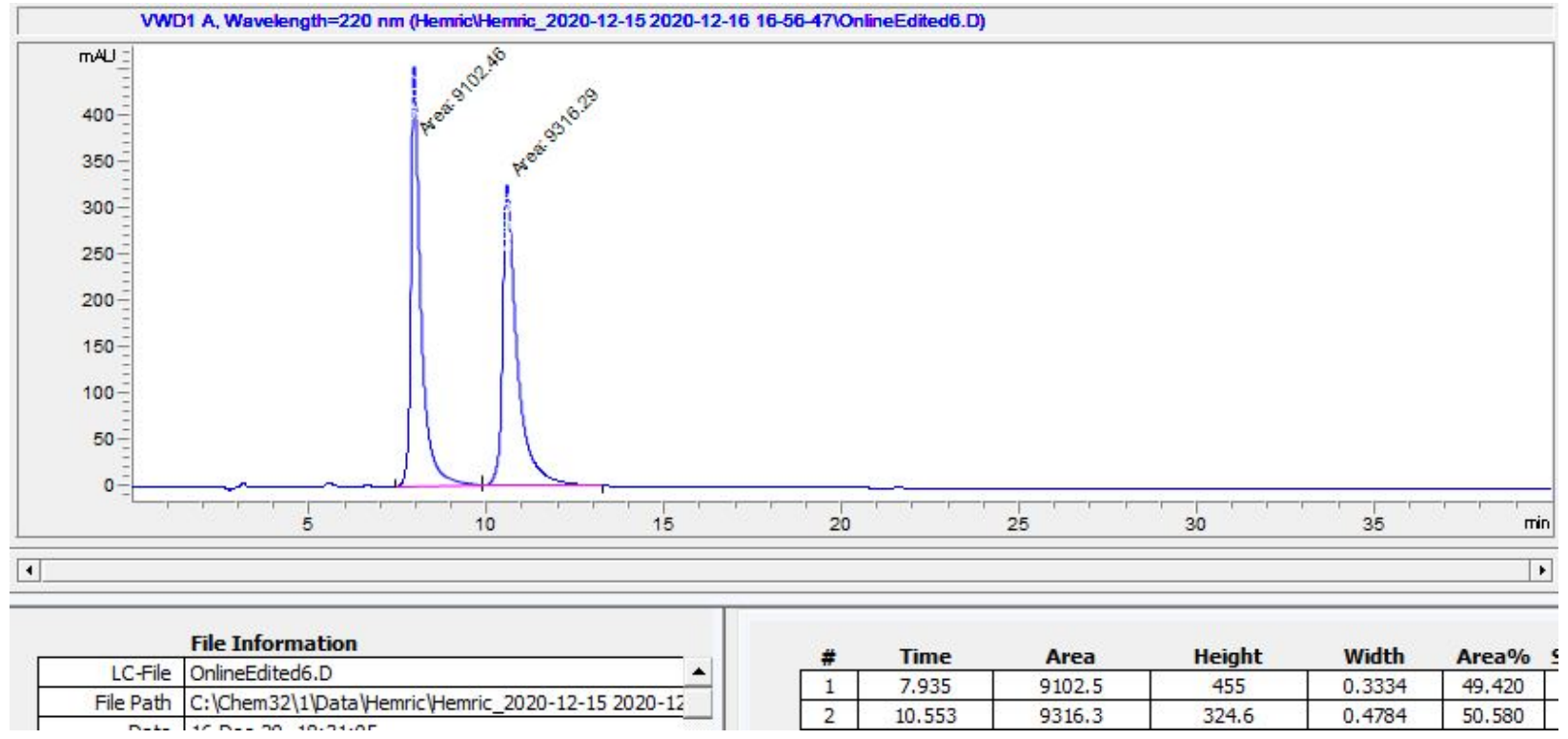

\section{4h- $(R, R)$}

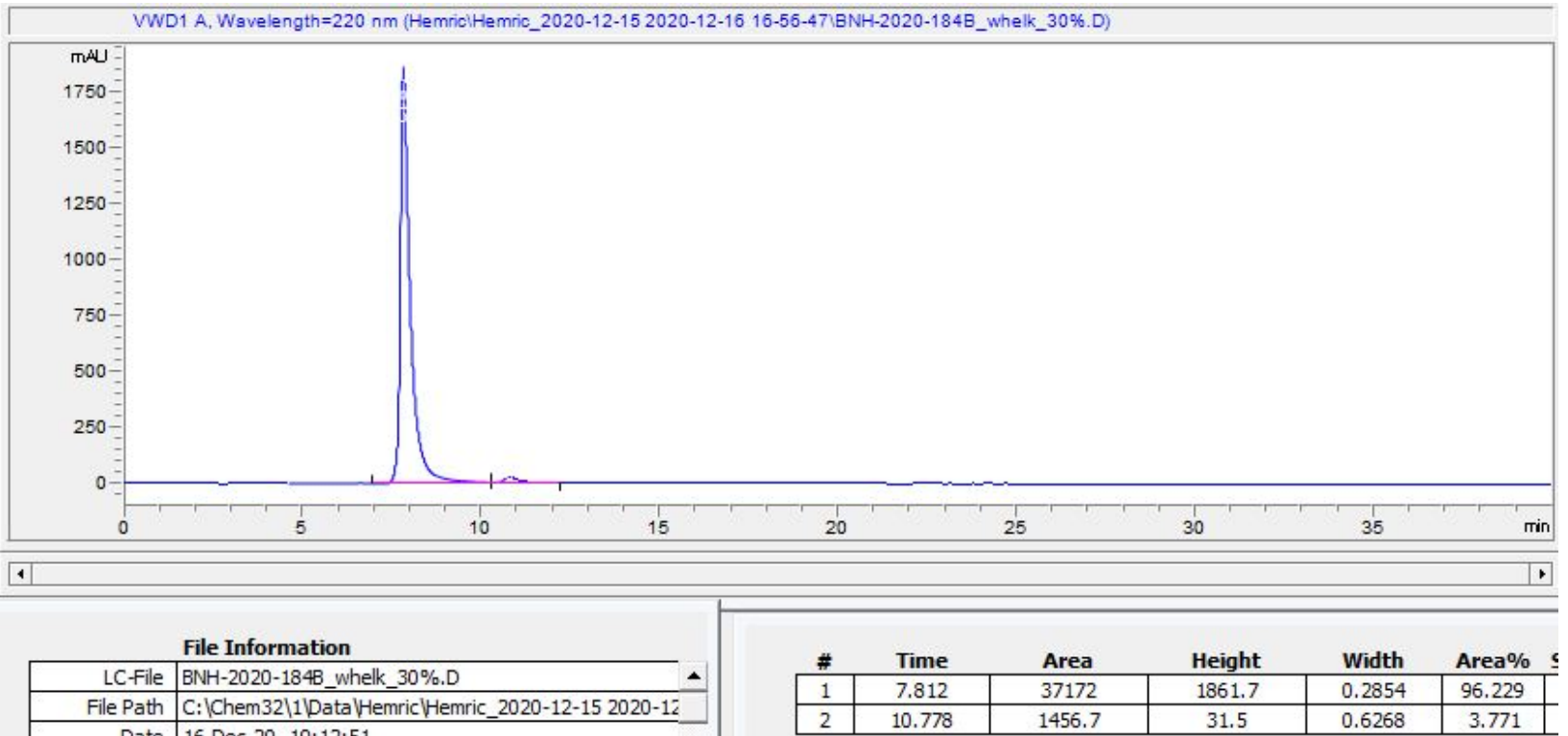


4i ${ }^{1} \mathrm{H}$ NMR (500 MHz, $\mathrm{CDCl}_{3}$ )

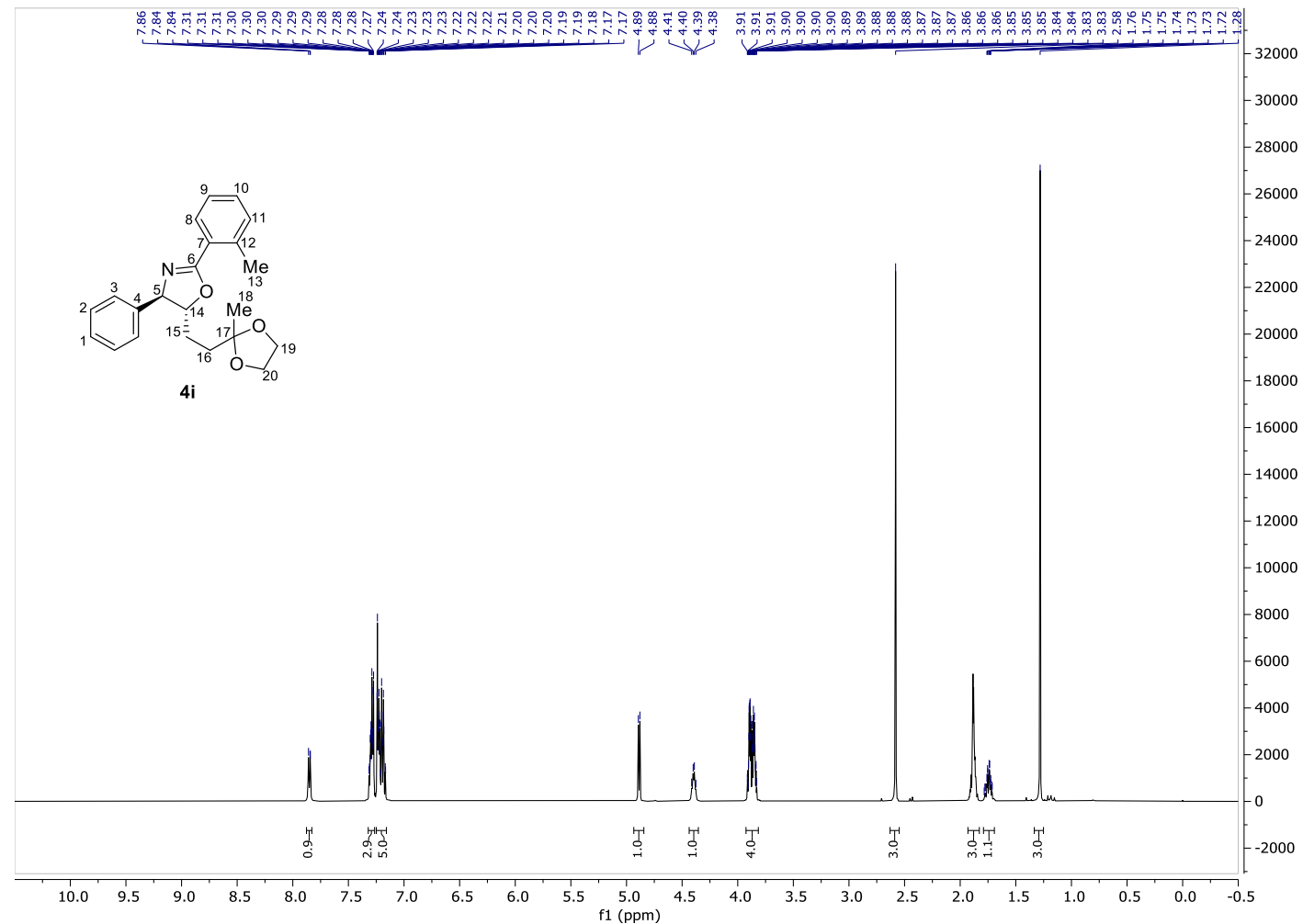

4i ${ }^{13} \mathrm{C}$ NMR (126 MHz, $\mathrm{CDCl}_{3}$ )

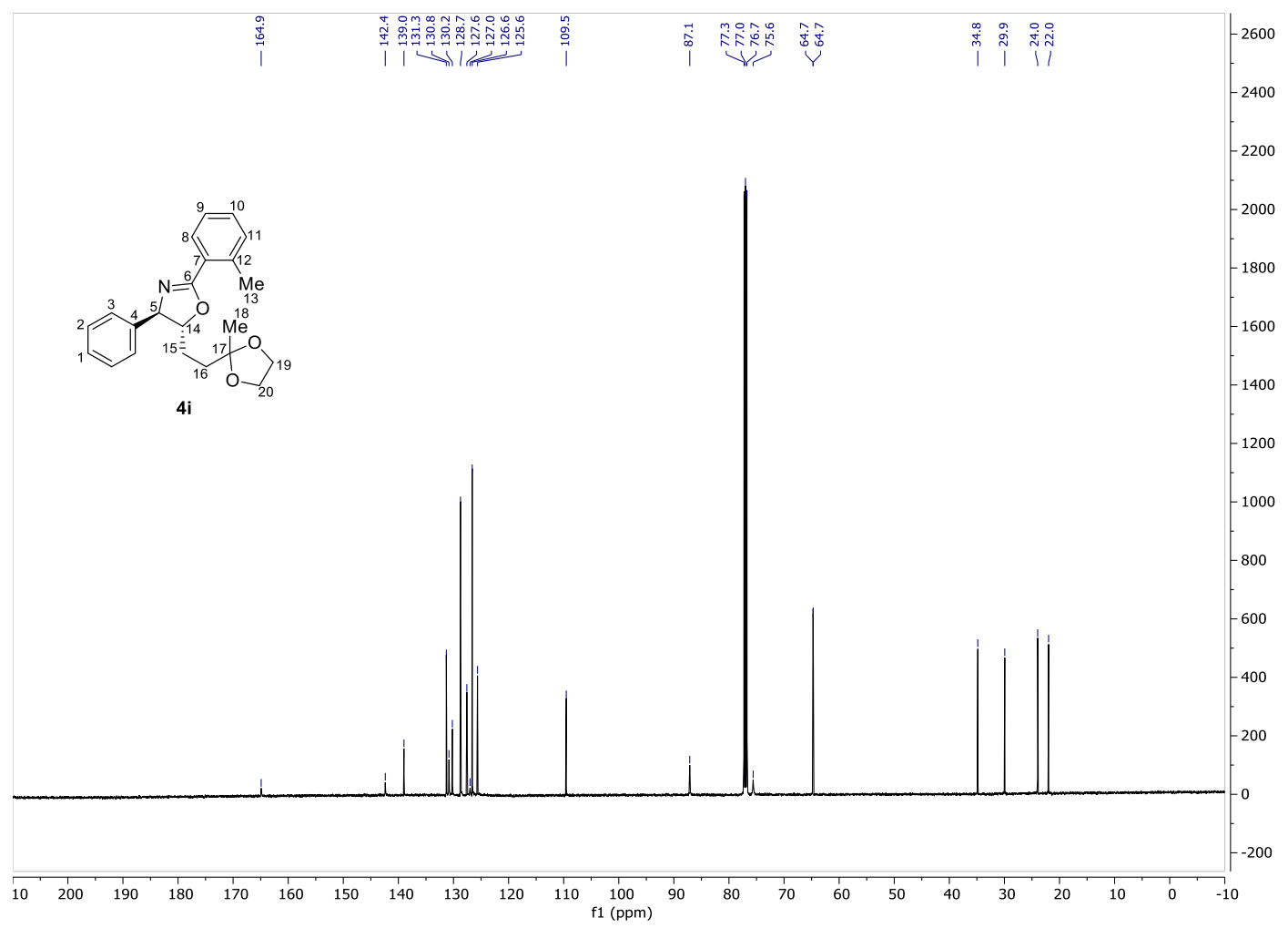




\section{4i-racemic}

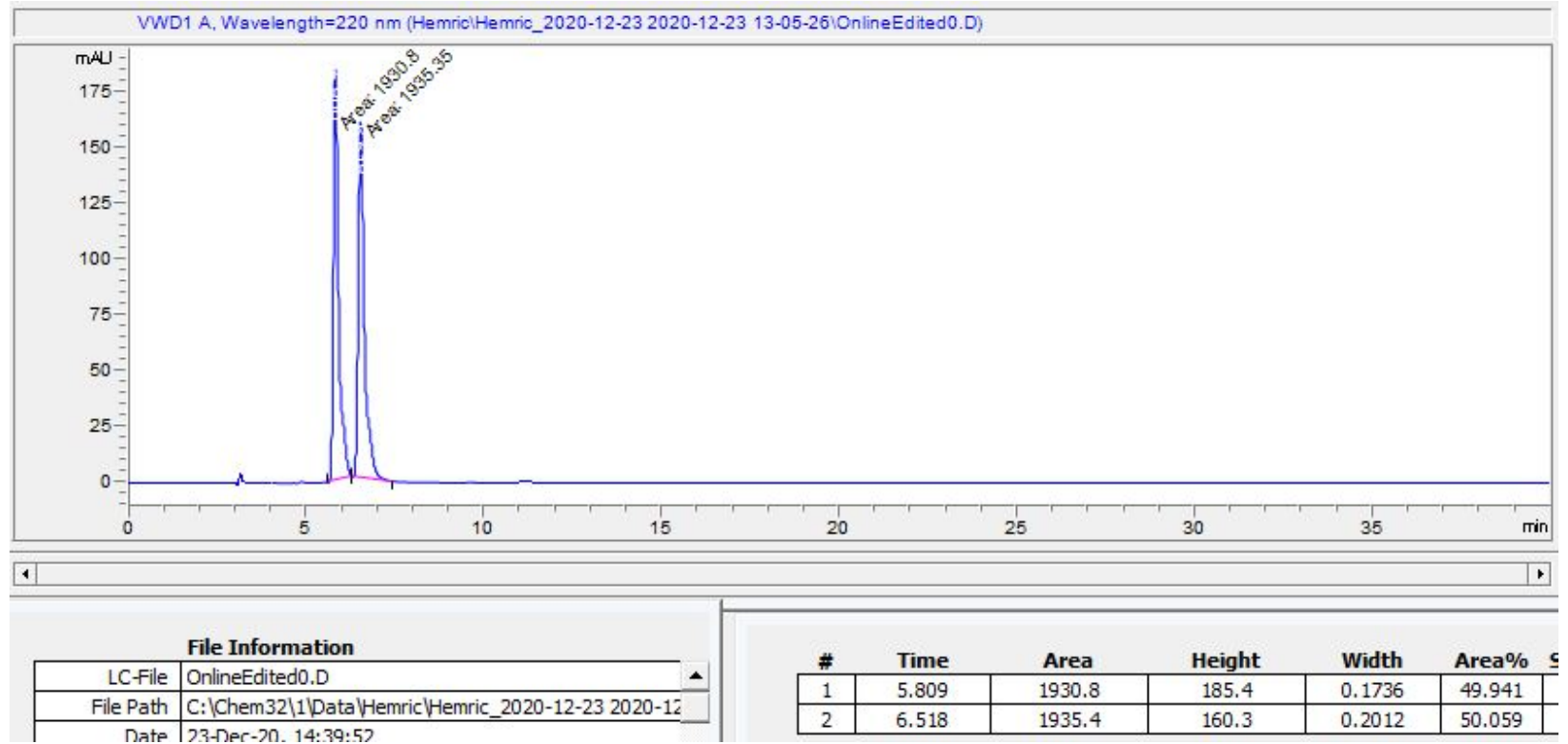

\section{$4 \mathbf{i}-(R, R)$}

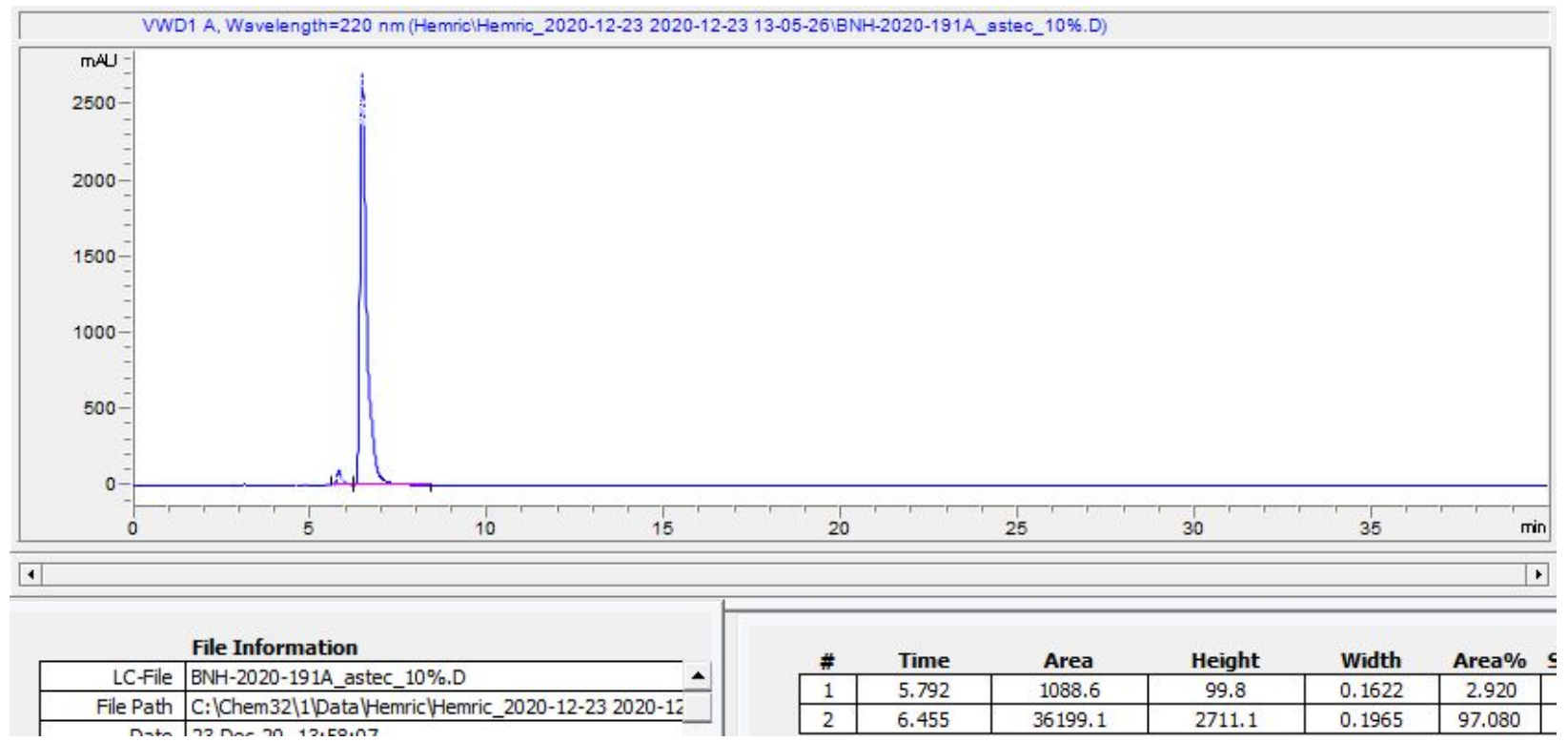


4j ${ }^{1} \mathrm{H}$ NMR (500 MHz, $\left.\mathrm{CDCl}_{3}\right)$

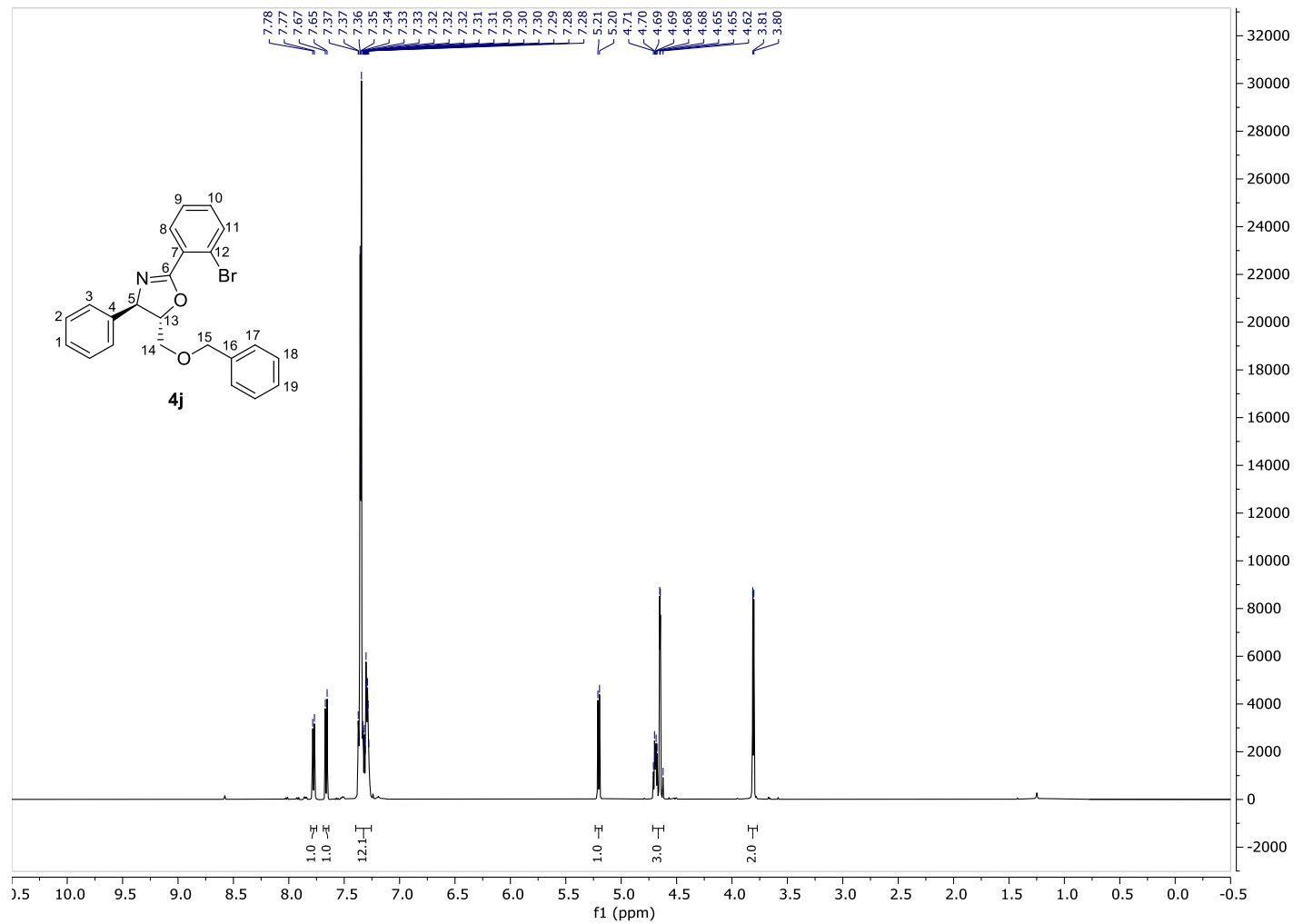

4j ${ }^{13} \mathrm{C}$ NMR (126 MHz, $\left.\mathrm{CDCl}_{3}\right)$

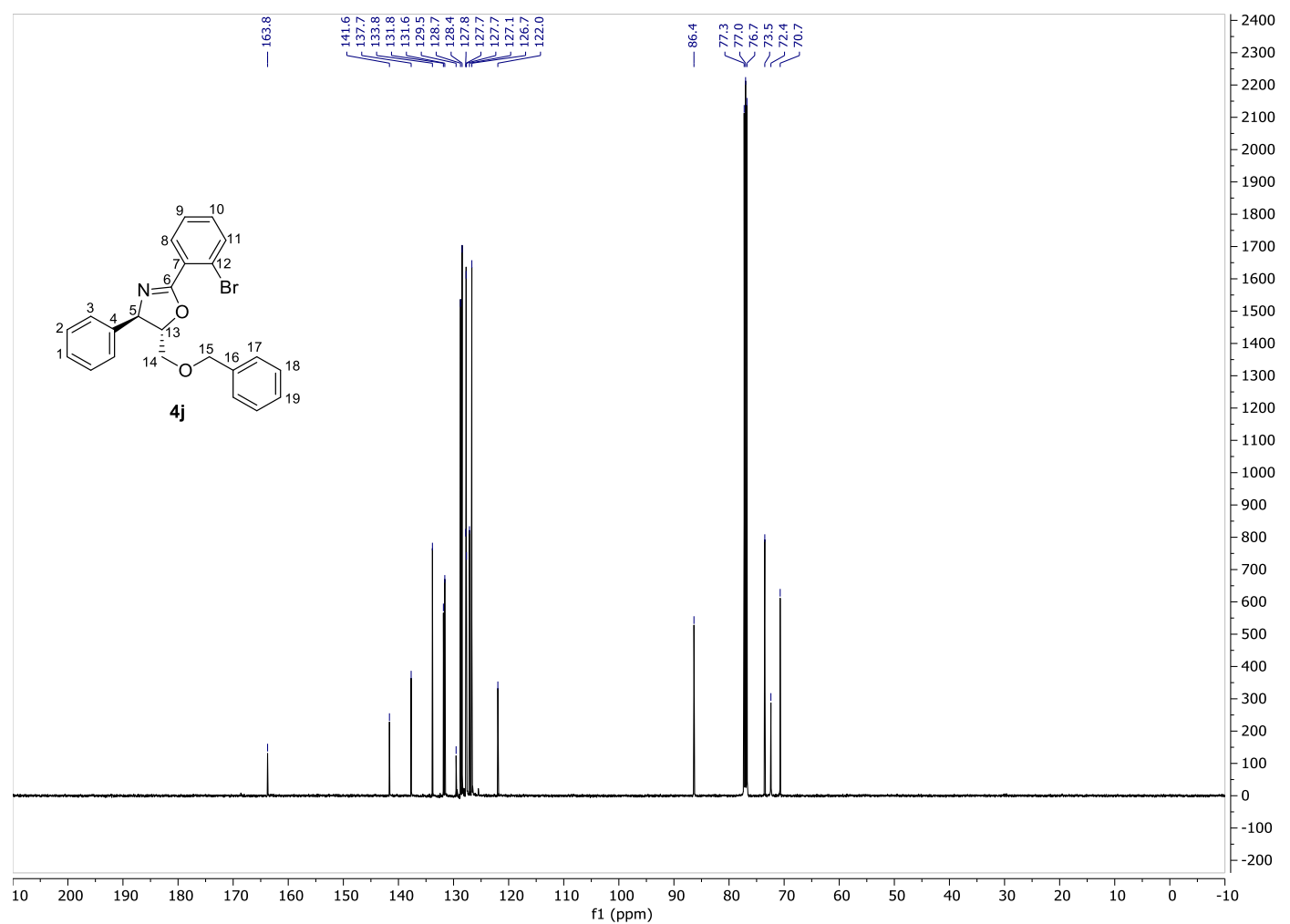


4j-racemic

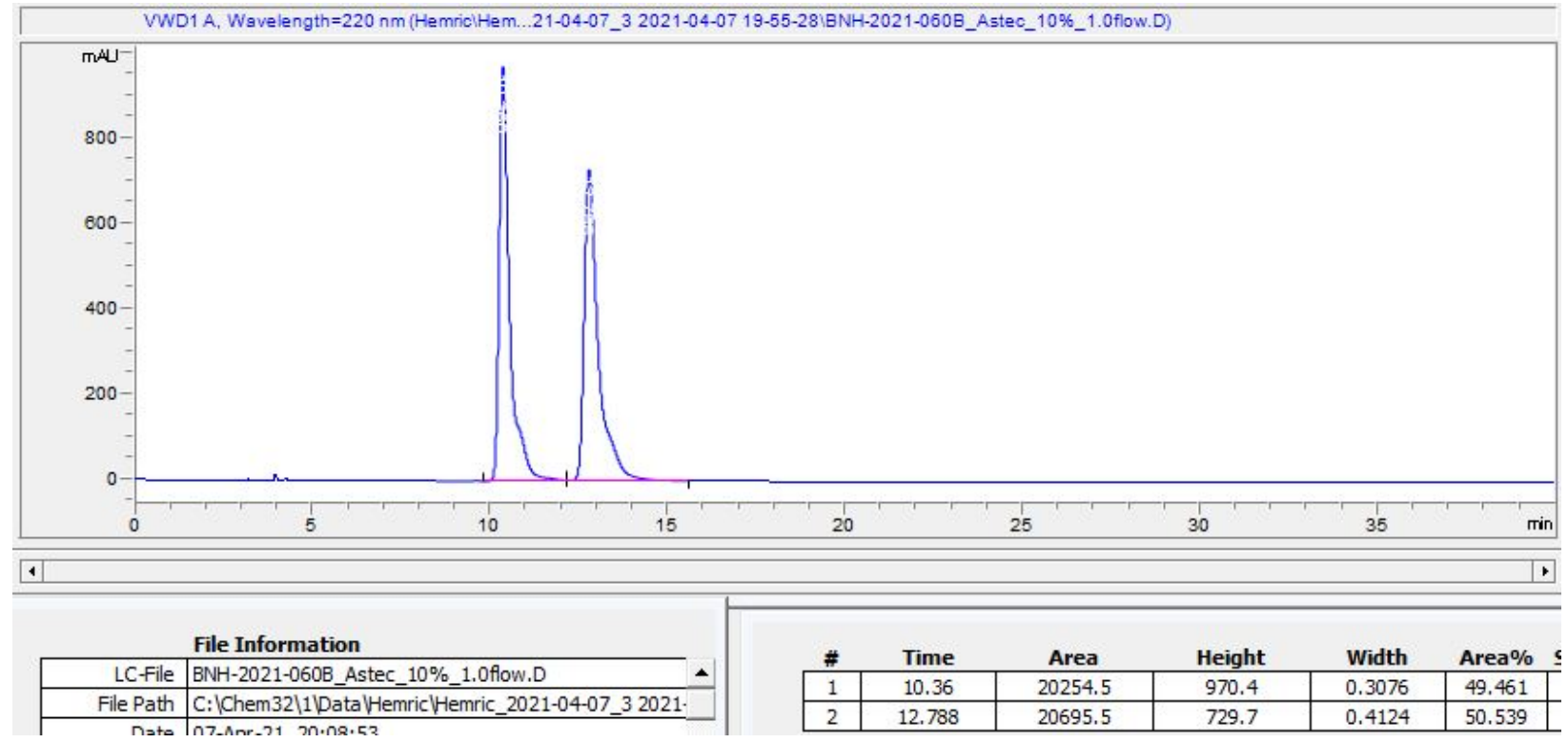

$\mathbf{4 j}-(R, R)$

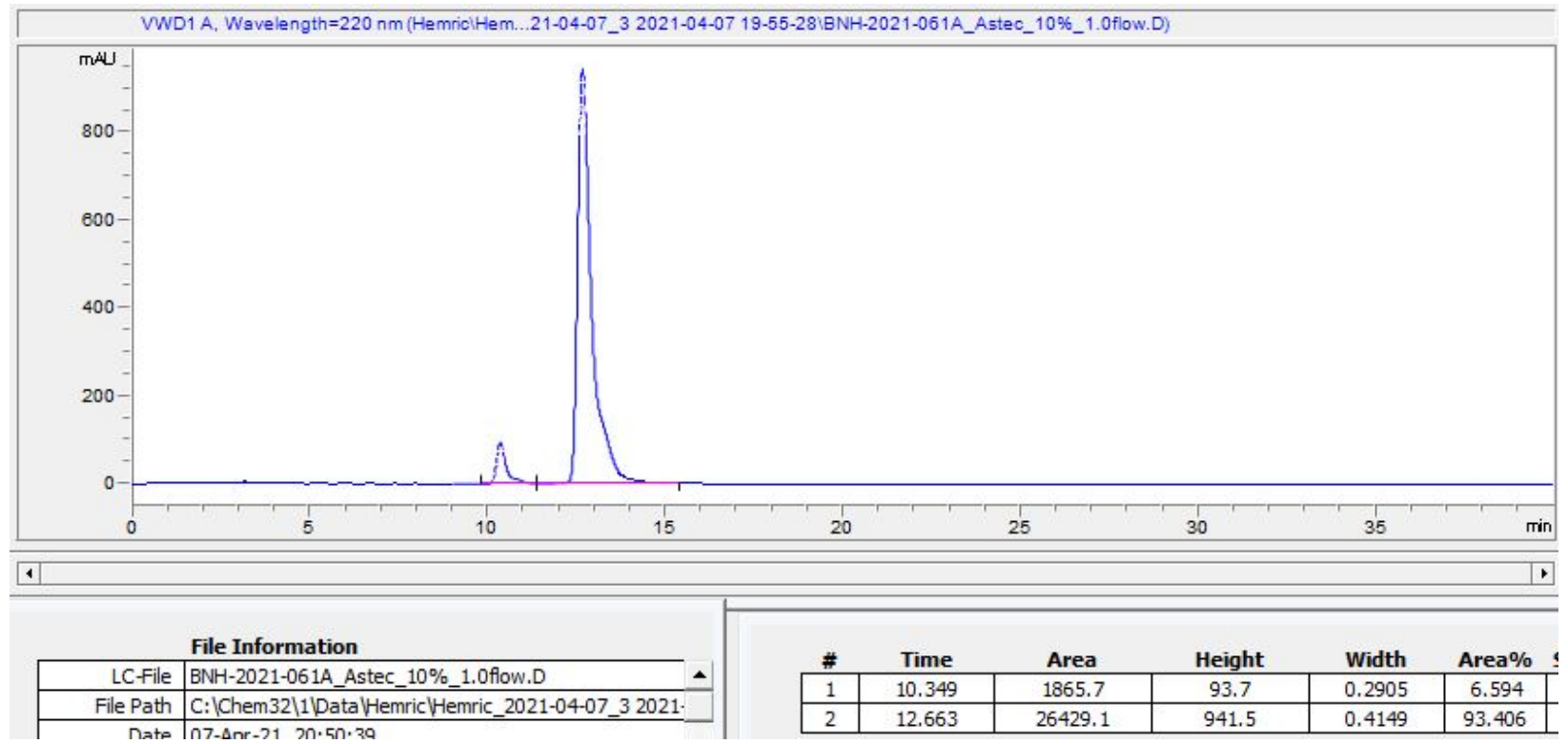


4k ${ }^{1} \mathrm{H}$ NMR (500 MHz, $\mathrm{CDCl}_{3}$ )

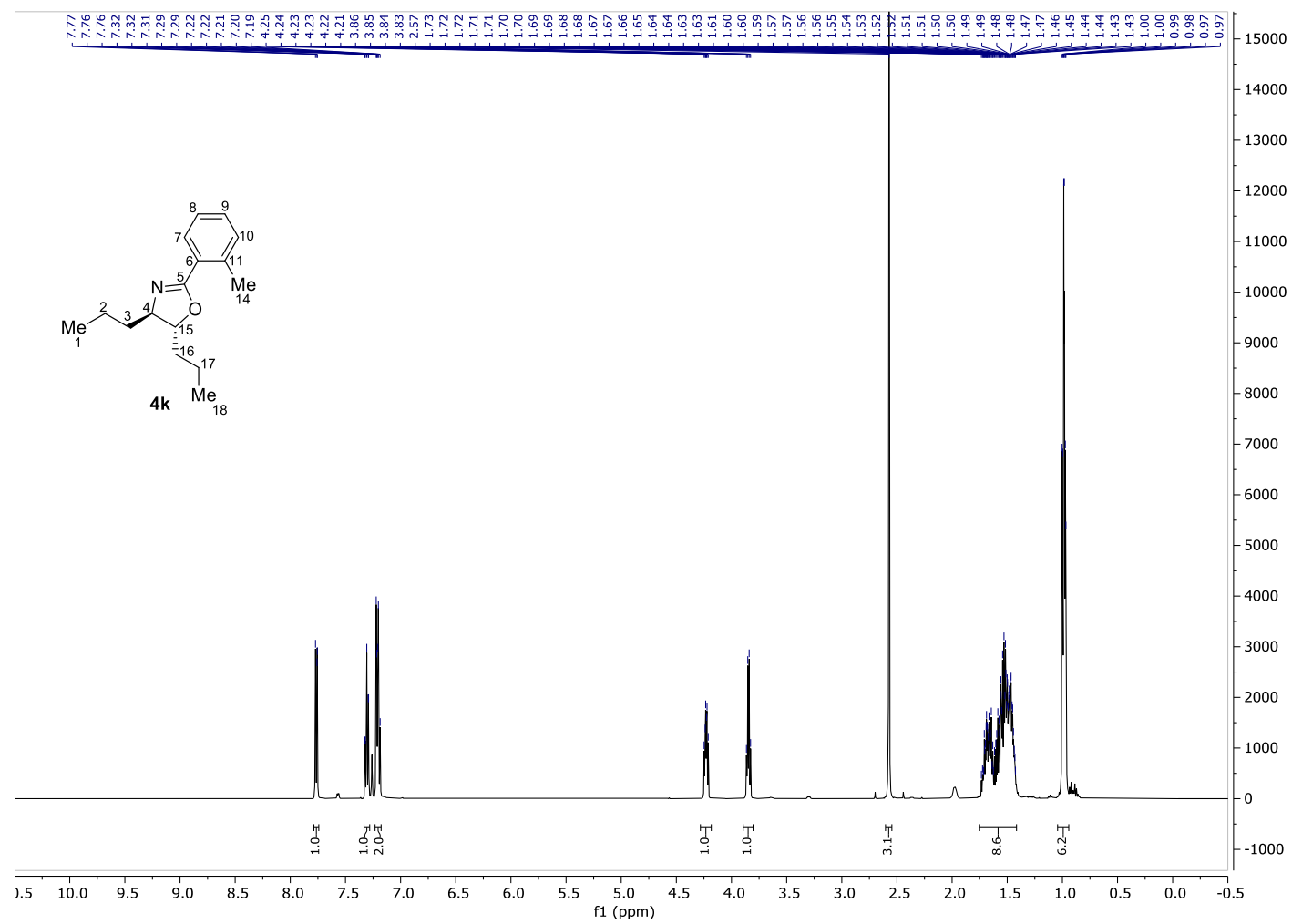

4k ${ }^{13} \mathrm{C}$ NMR (126 MHz, $\mathrm{CDCl}_{3}$ )

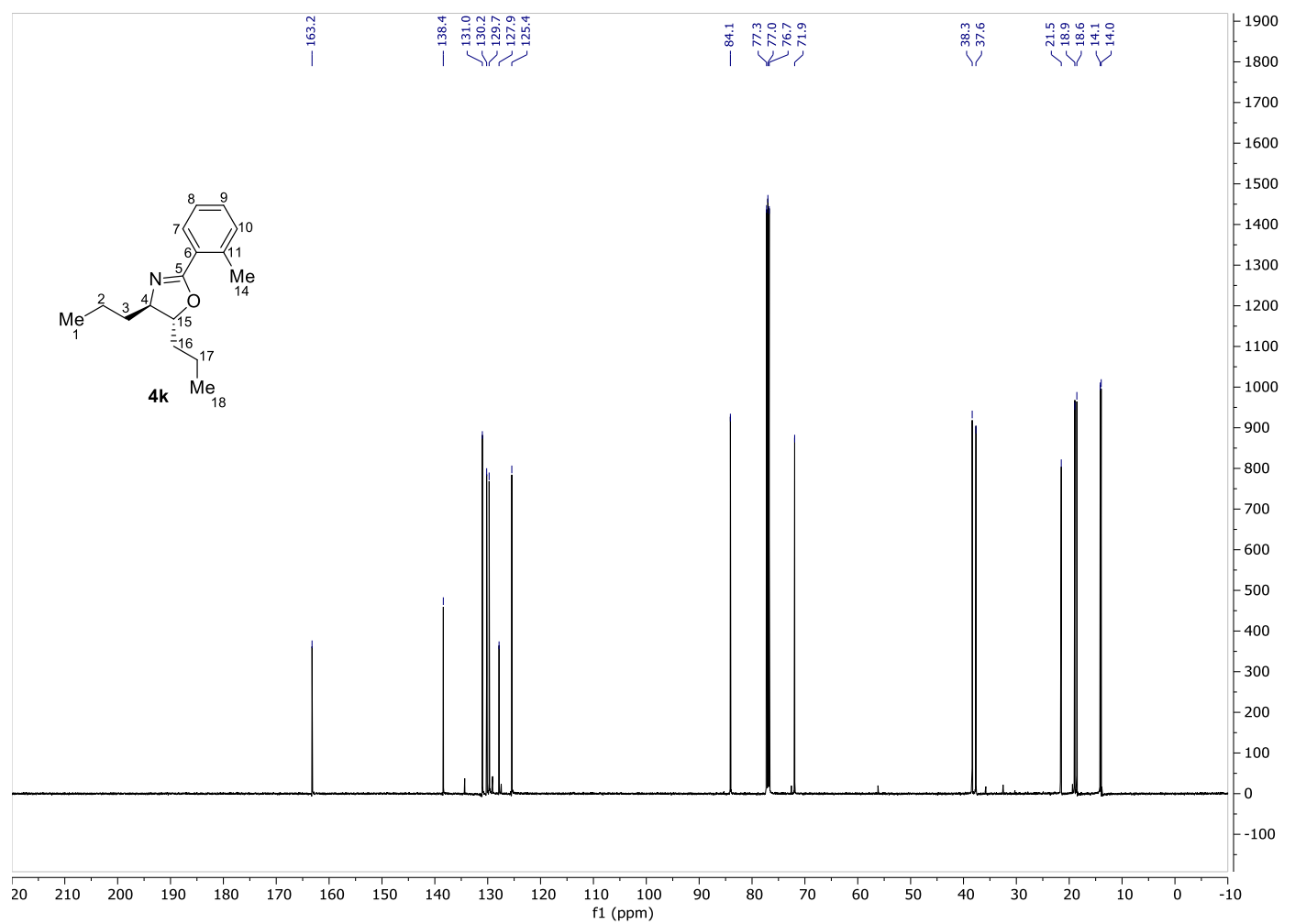




\section{4k-racemic}

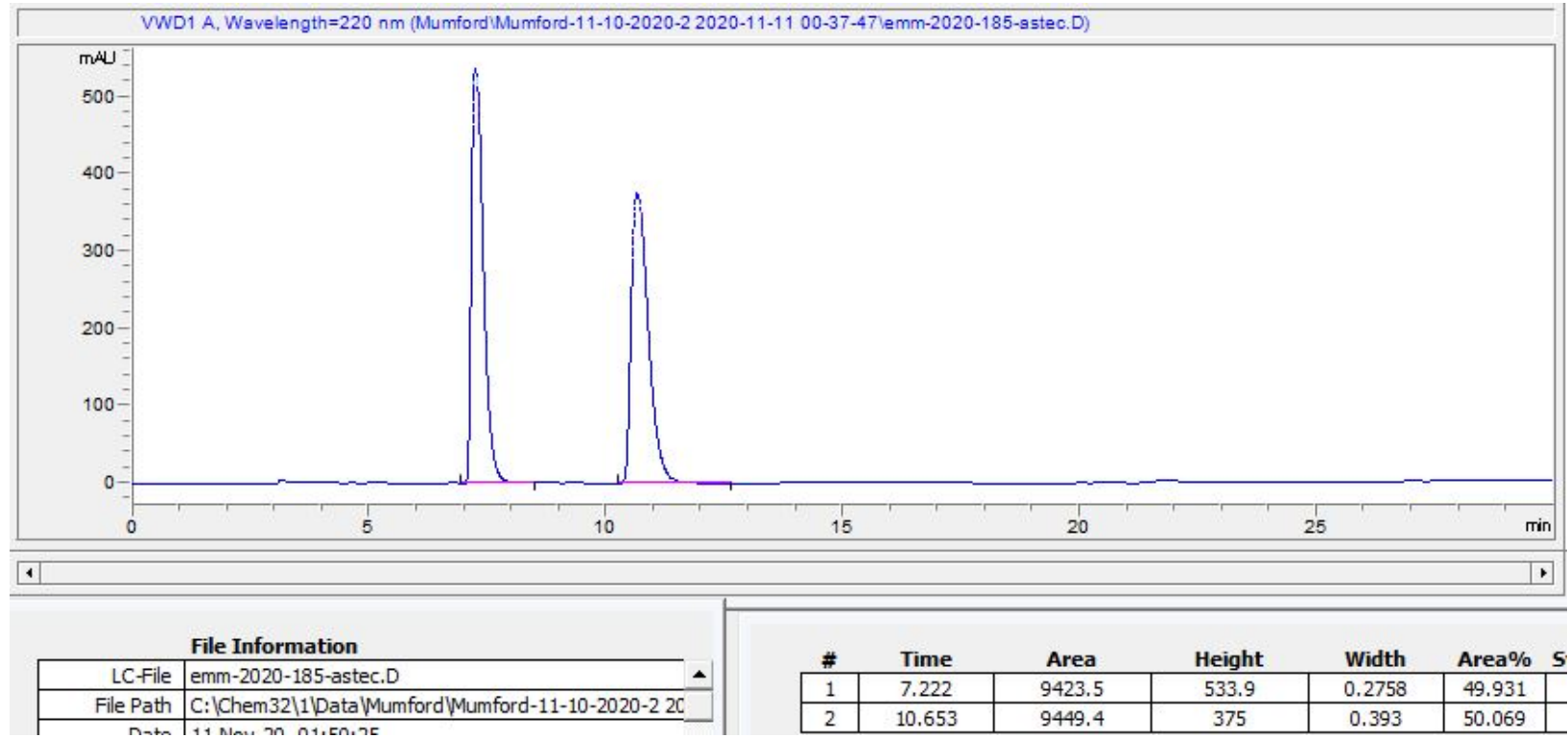

\section{$4 \mathbf{k}-(R, R)$}

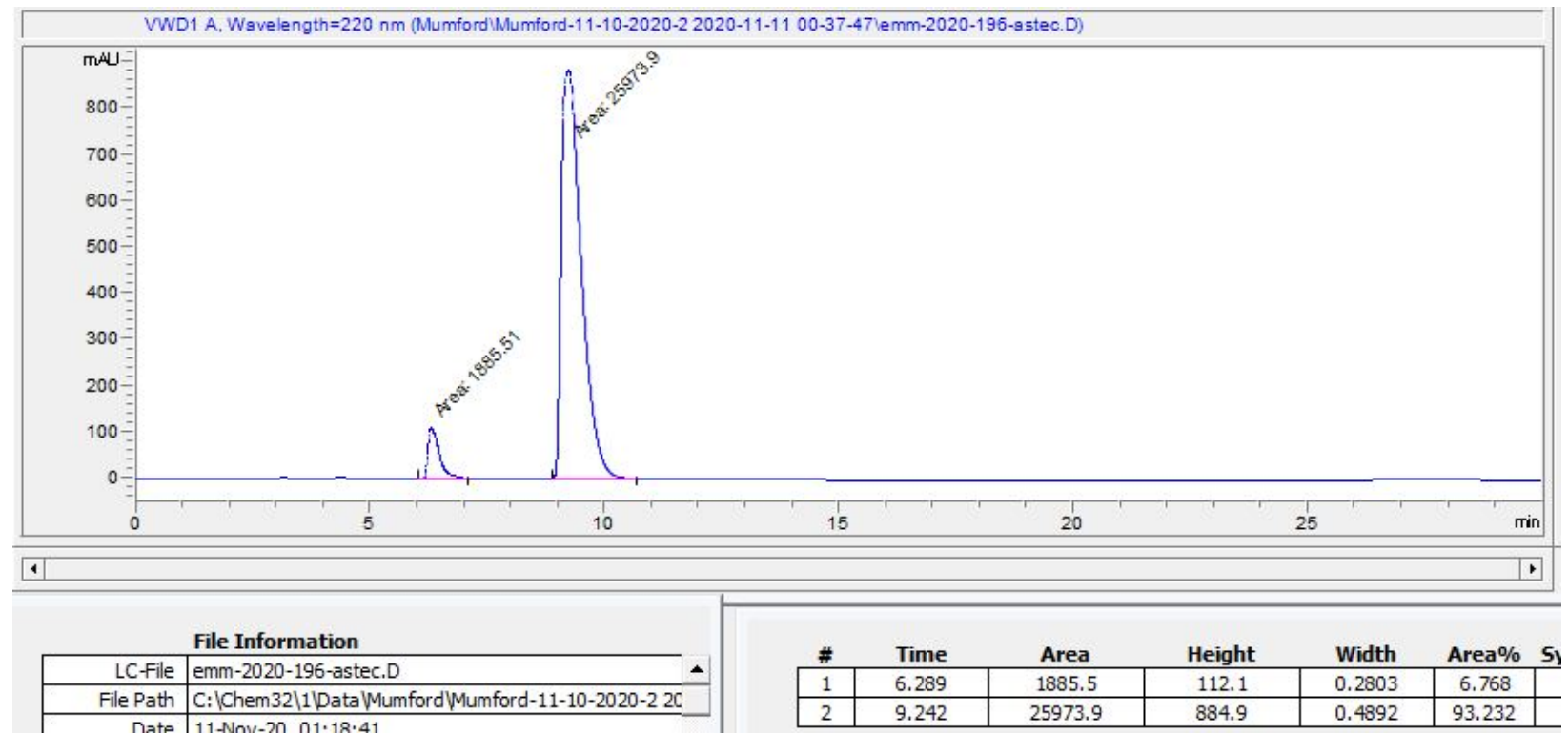


$4{ }^{1} \mathrm{H}$ NMR (500 MHz, $\left.\mathrm{CDCl}_{3}\right)$

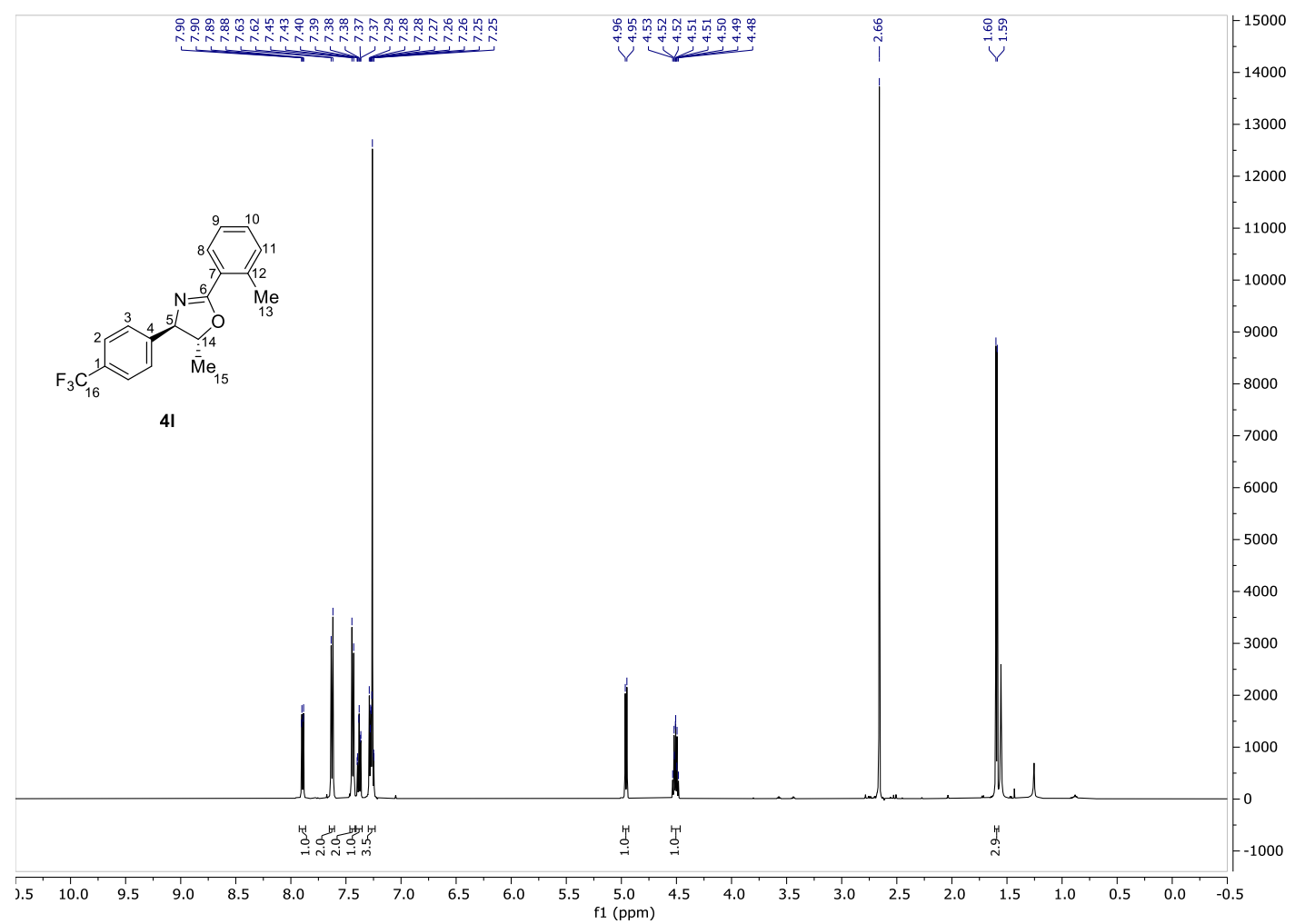

$4{ }^{13} \mathrm{C}$ NMR (126 MHz, $\mathrm{CDCl}_{3}$ )

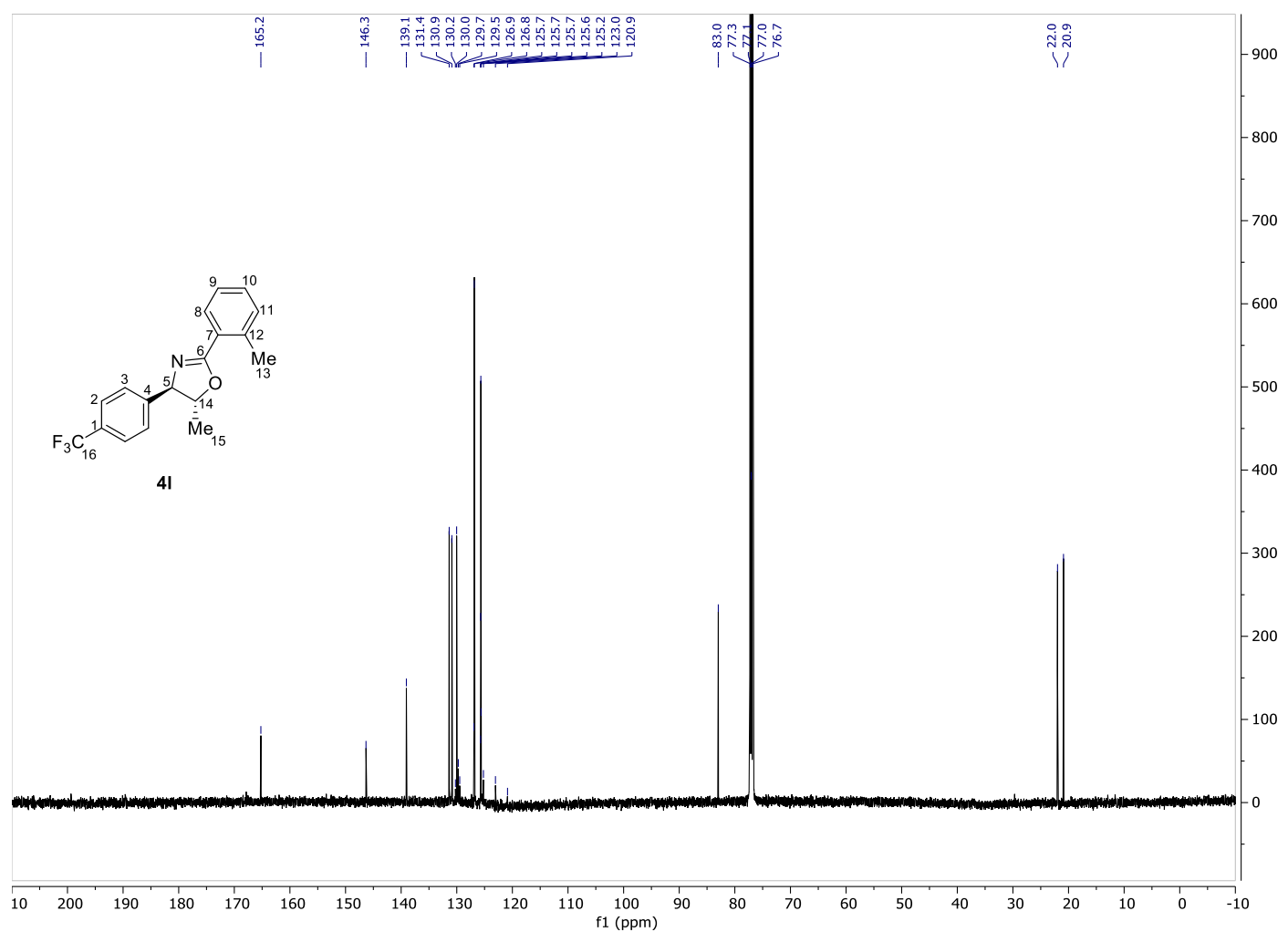


4l ${ }^{19} \mathrm{~F}$ NMR (471 MHz, $\mathrm{CDCl}_{3}$ with $\mathrm{C}_{6} \mathrm{~F}_{5}$ as standard)

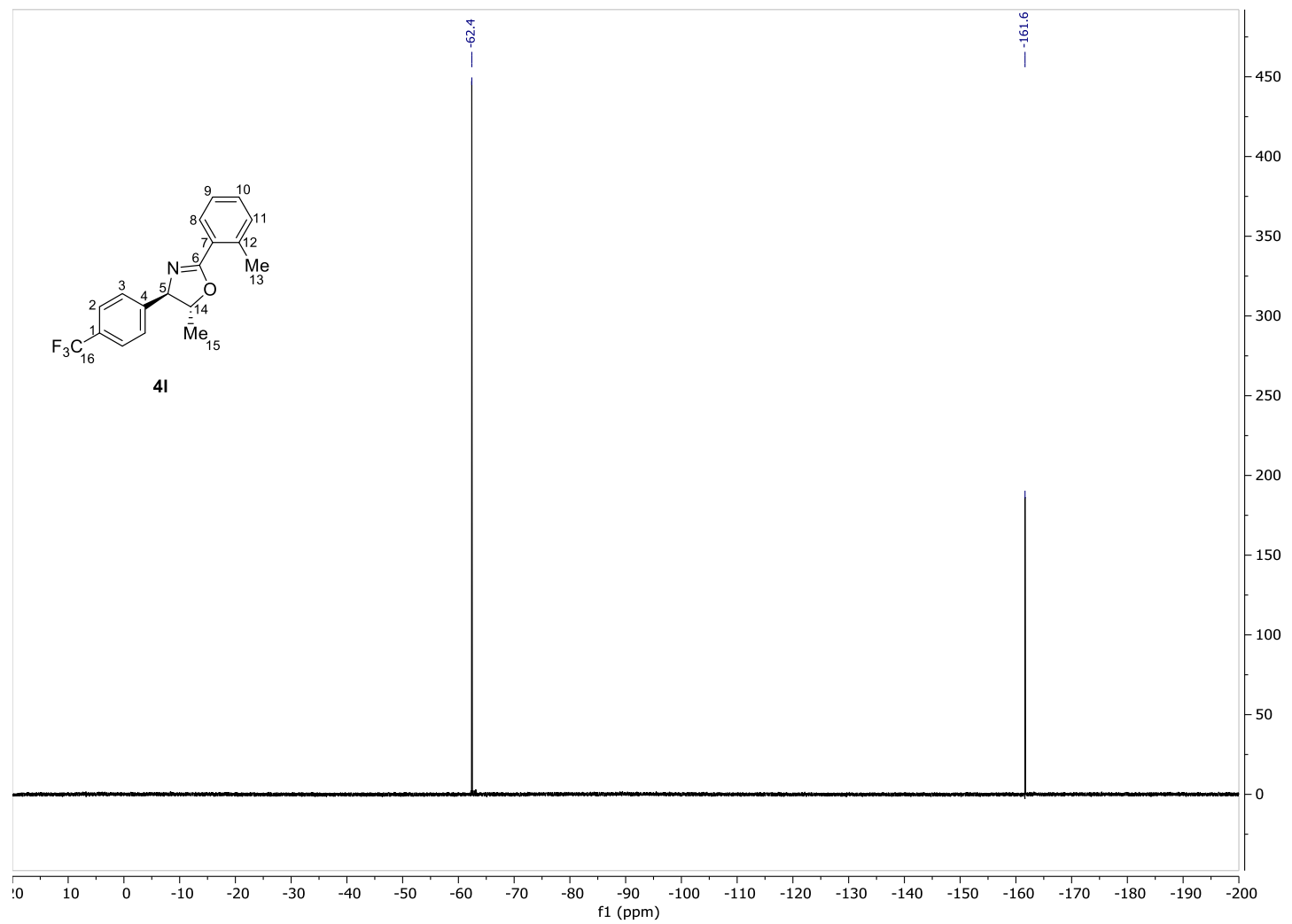




\section{4l-racemic}

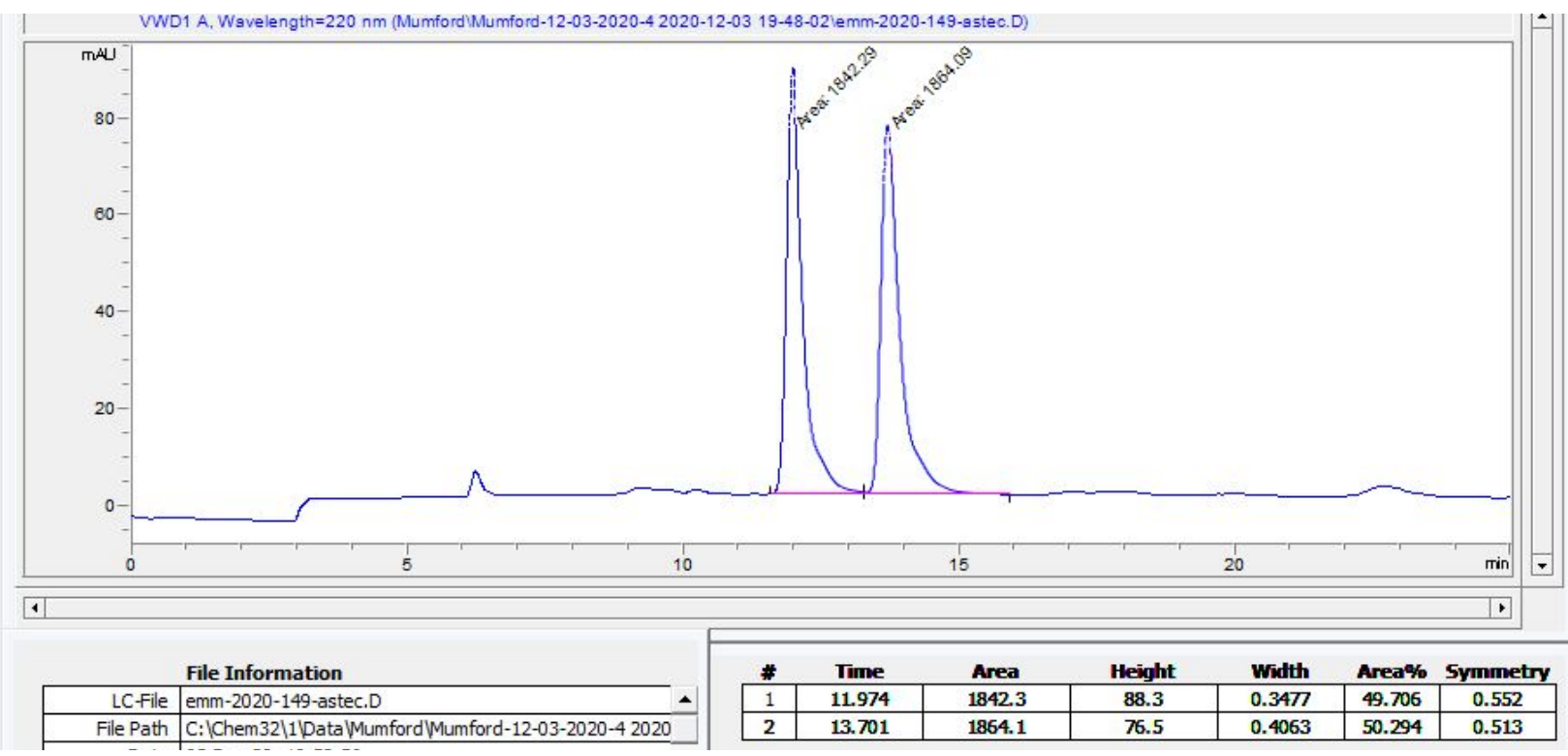

\section{4l- $(R, R)$}

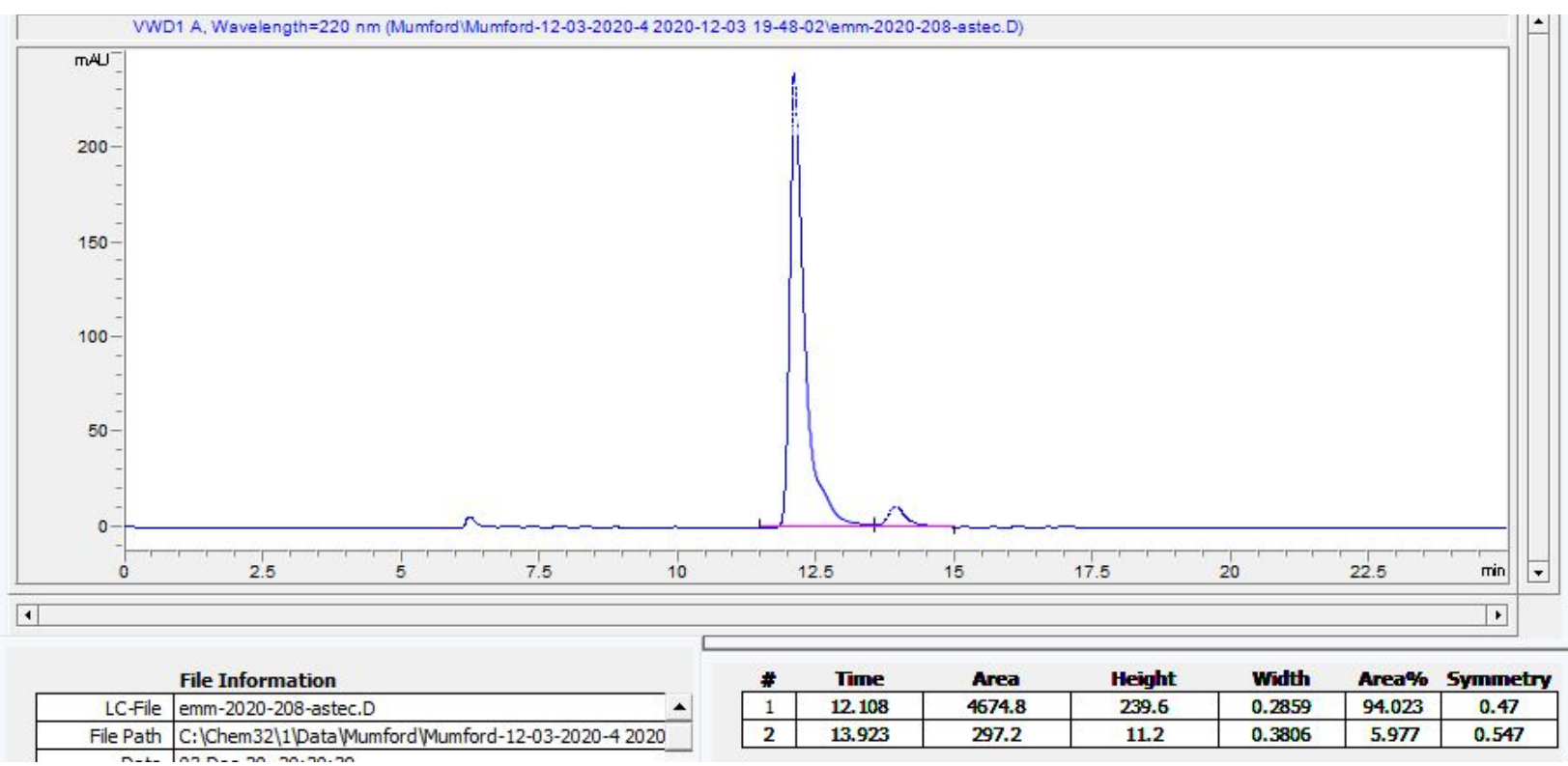


4m ${ }^{1} \mathrm{H}$ NMR (500 MHz, $\mathrm{CDCl}_{3}$ )

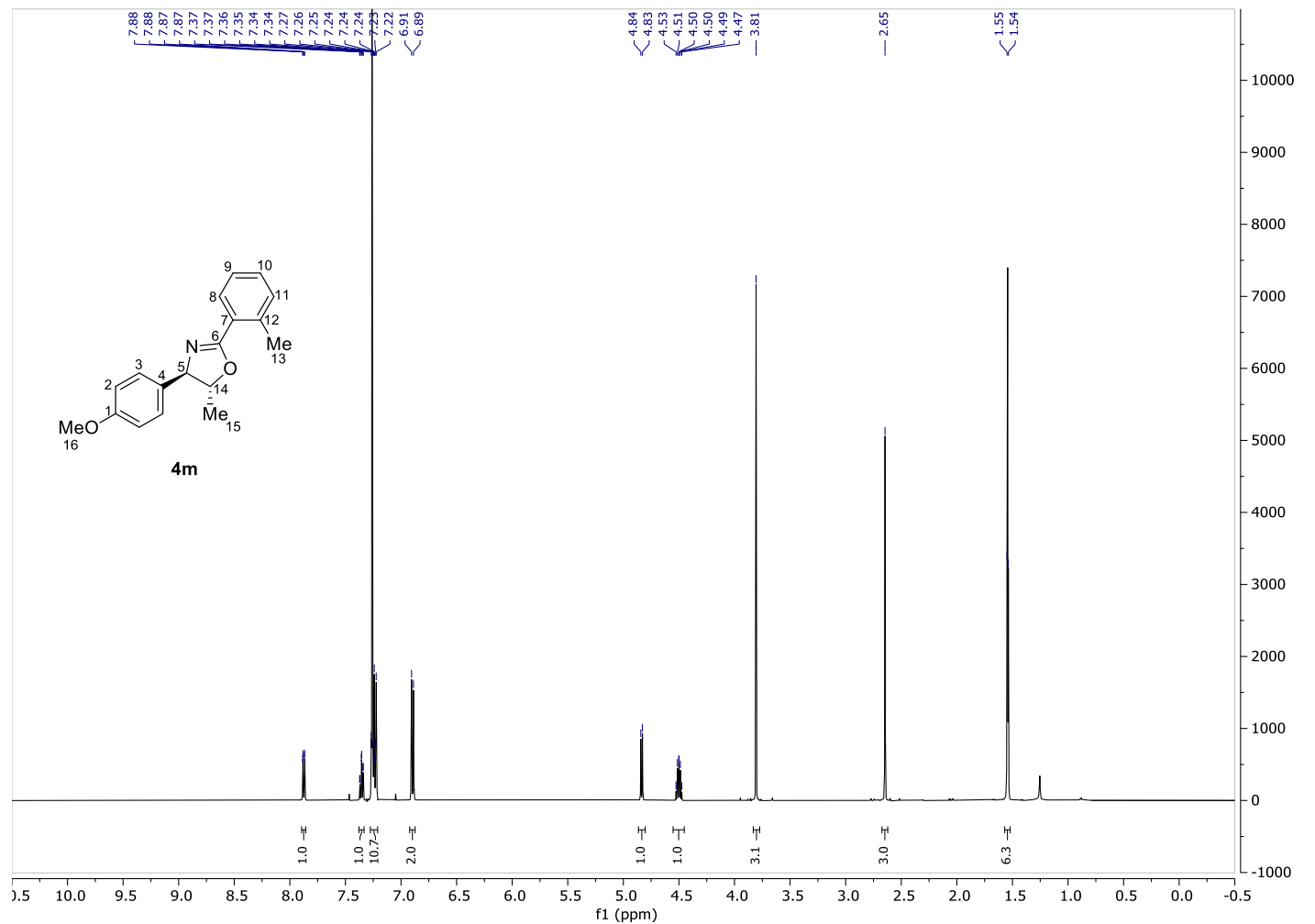

4m ${ }^{13} \mathrm{C}$ NMR (126 MHz, $\left.\mathrm{CDCl}_{3}\right)$

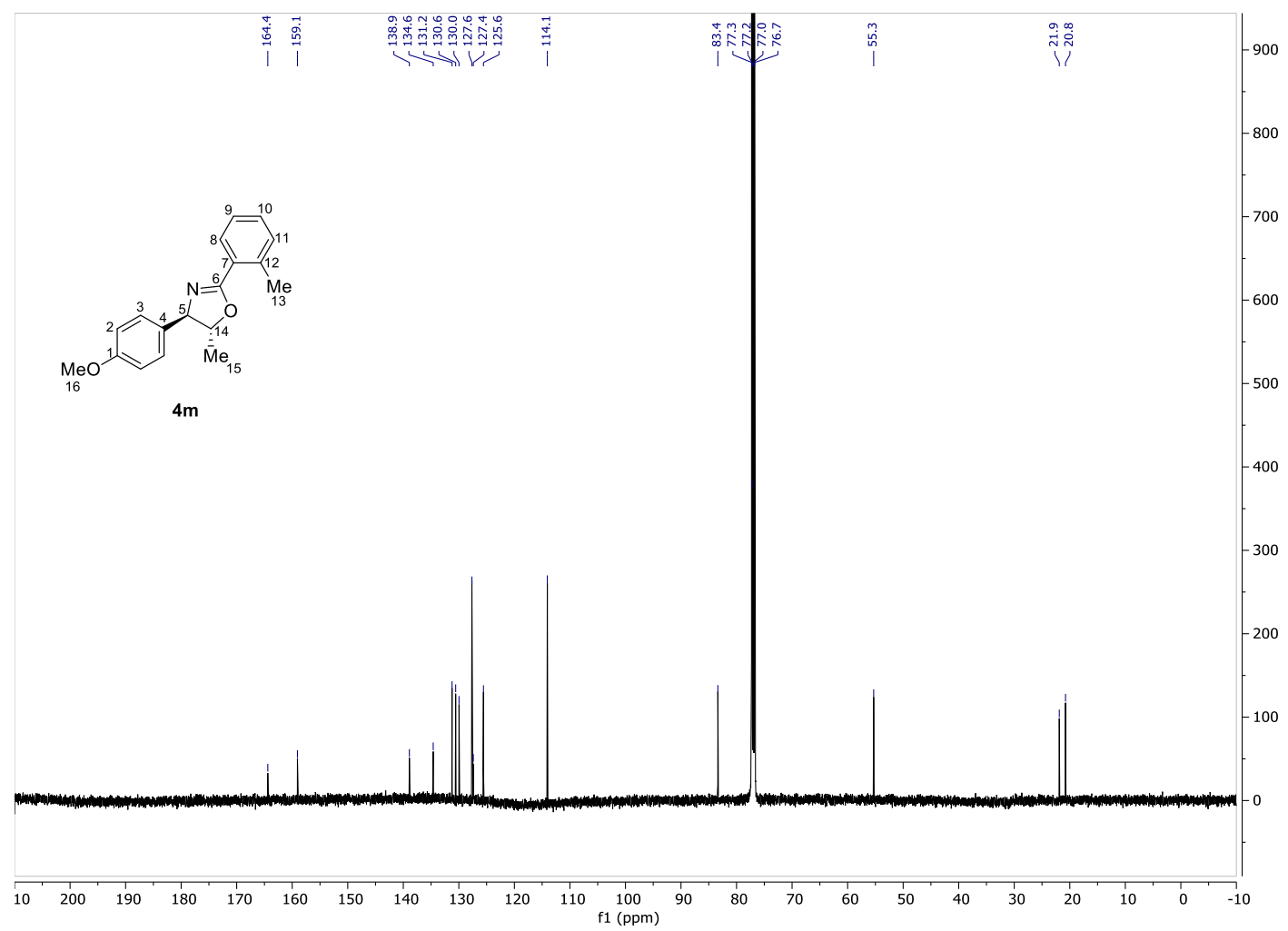




\section{4m-racemic}

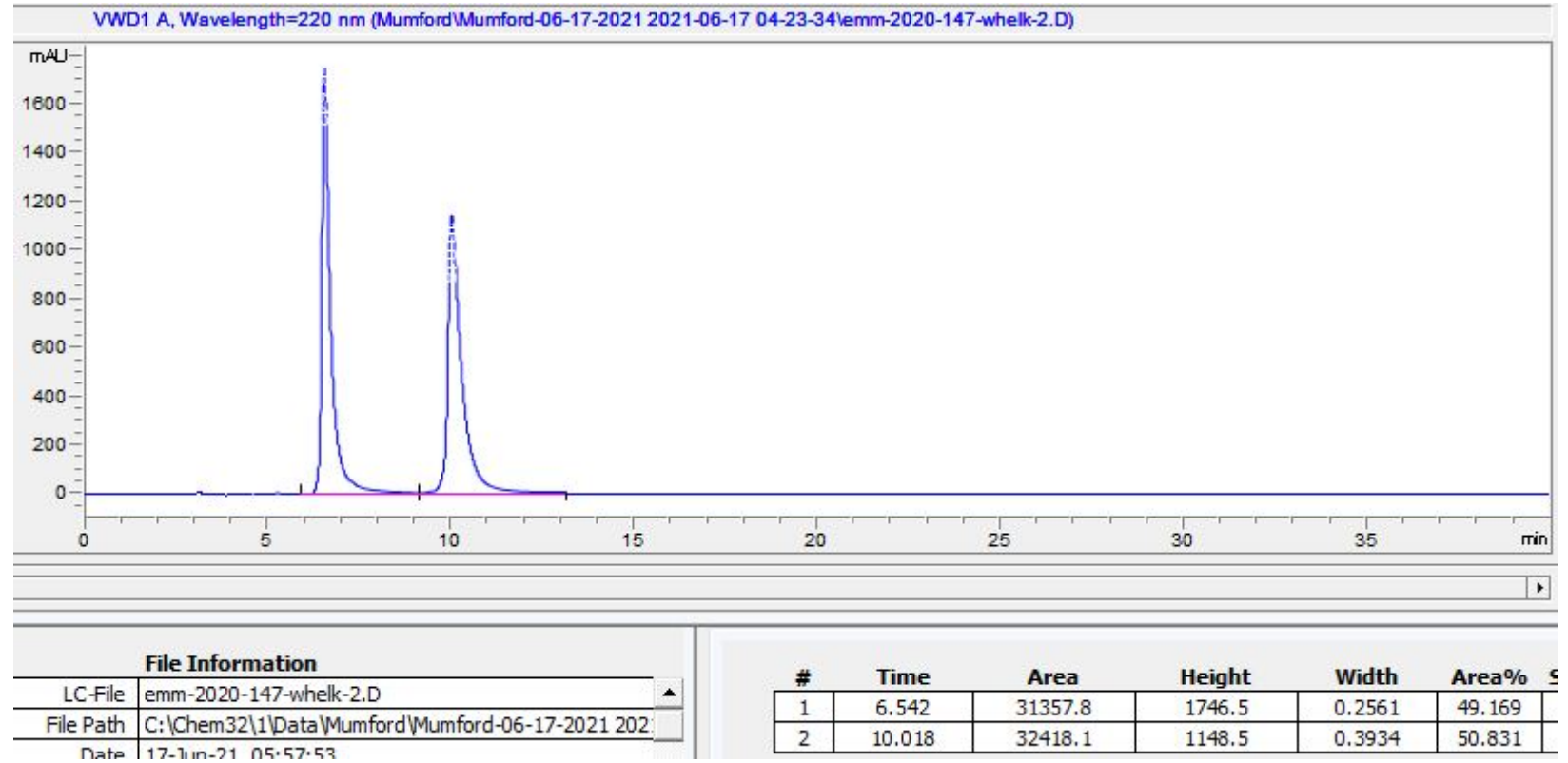

\section{4m- $(R, R)$}

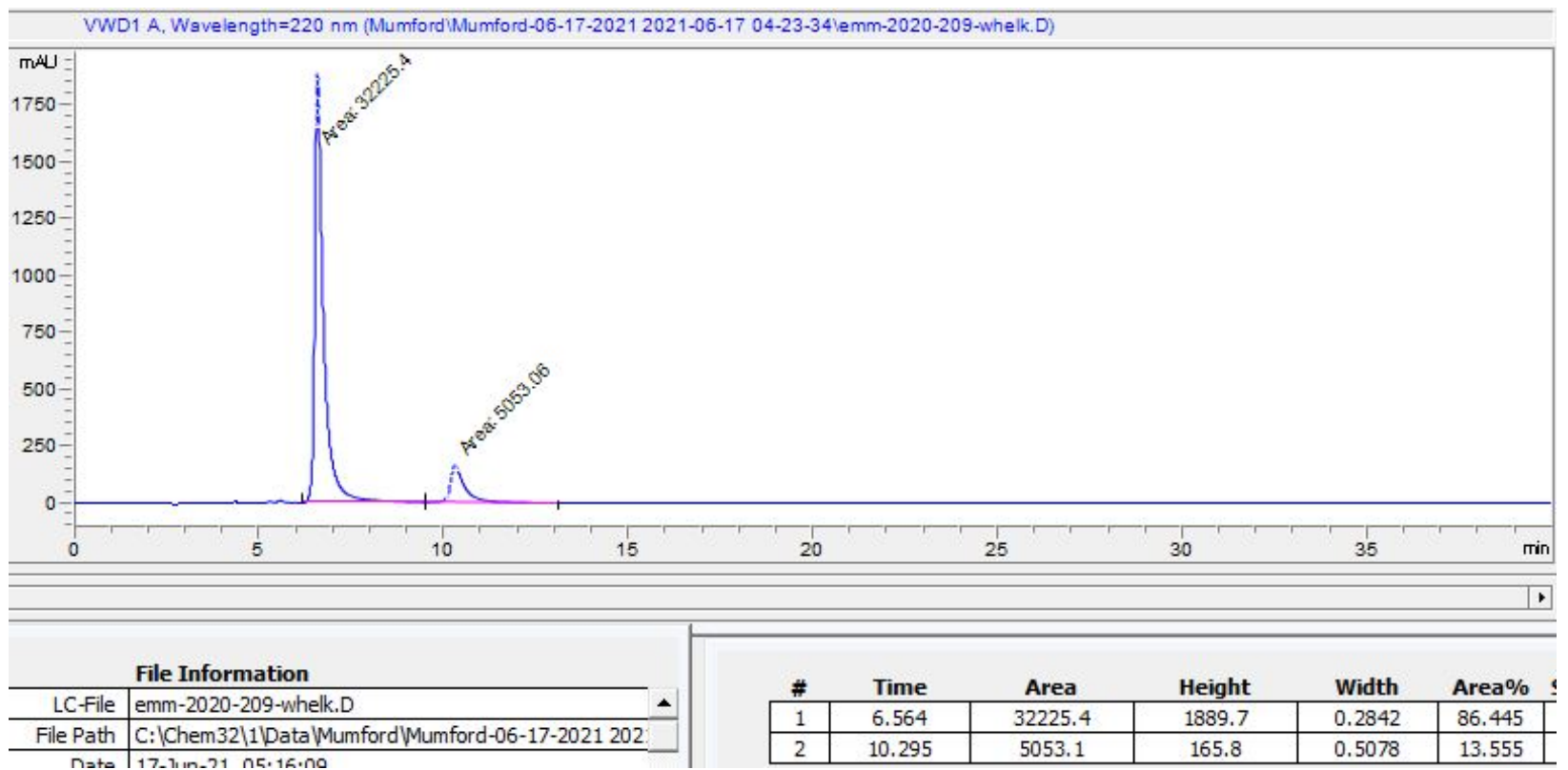


4n ${ }^{1} \mathrm{H}$ NMR $\left(500 \mathrm{MHz}, \mathrm{CDCl}_{3}\right)$

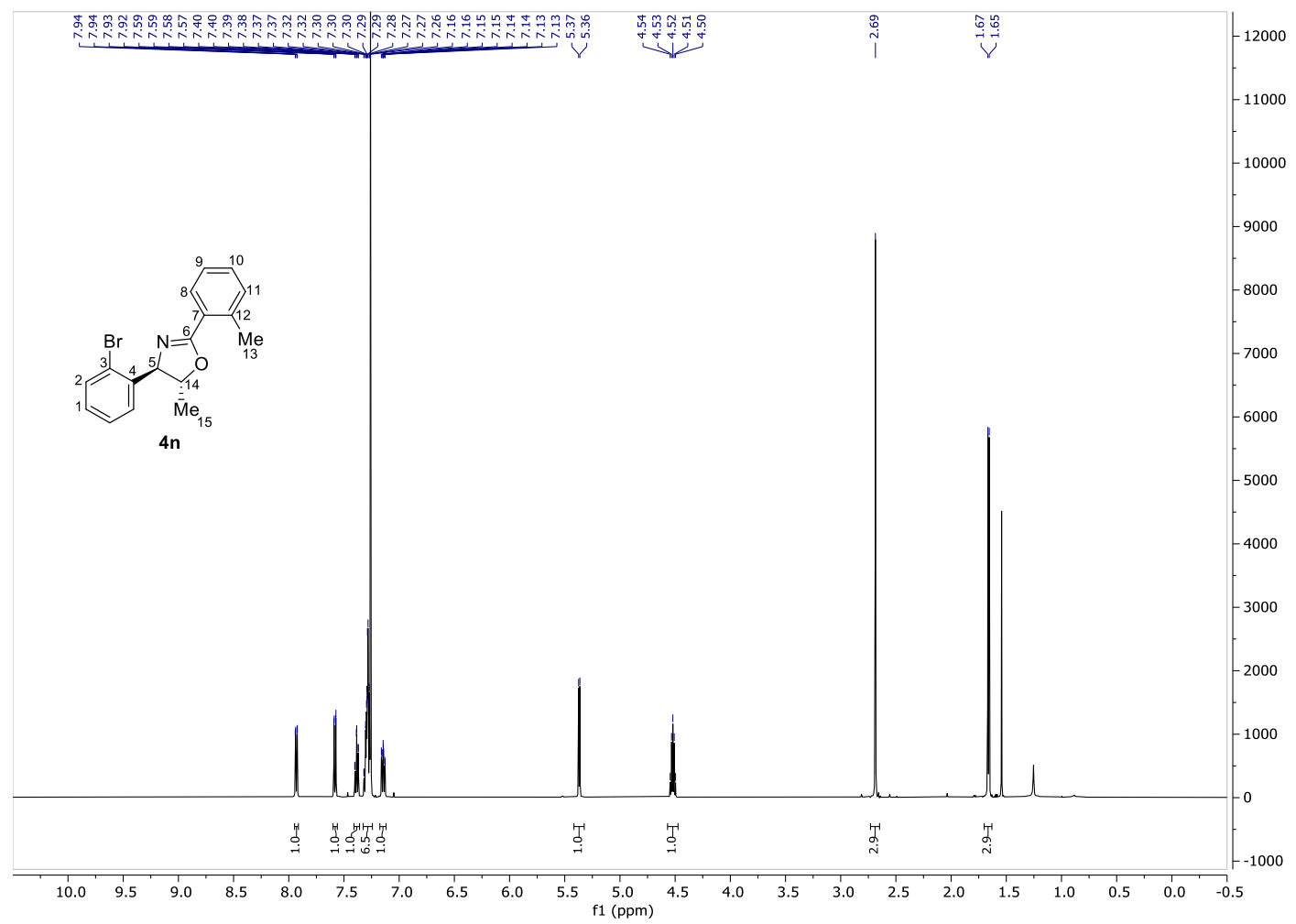

4n ${ }^{13} \mathrm{C}$ NMR (126 MHz, $\mathrm{CDCl}_{3}$ )

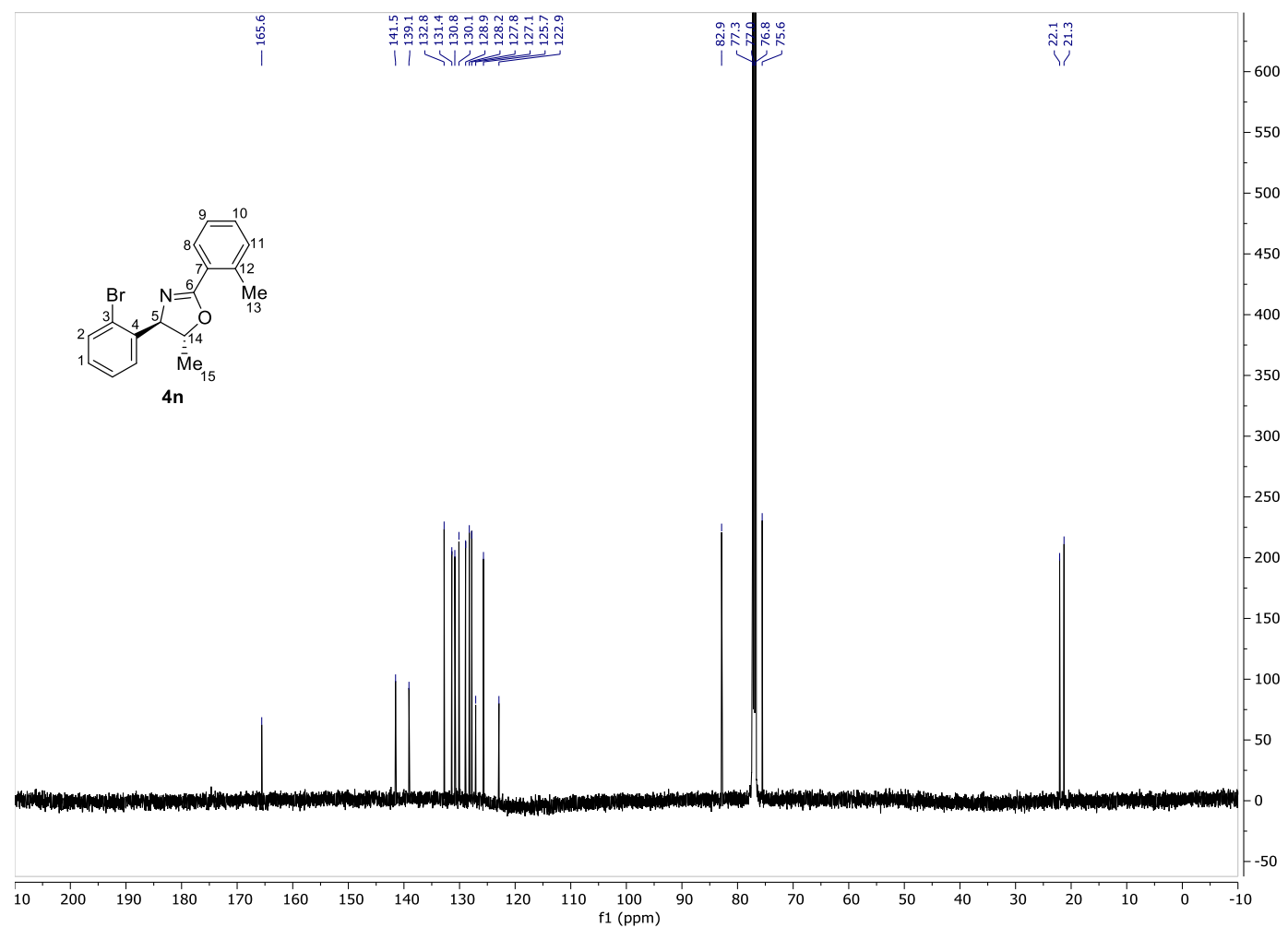




\section{4n-racemic}

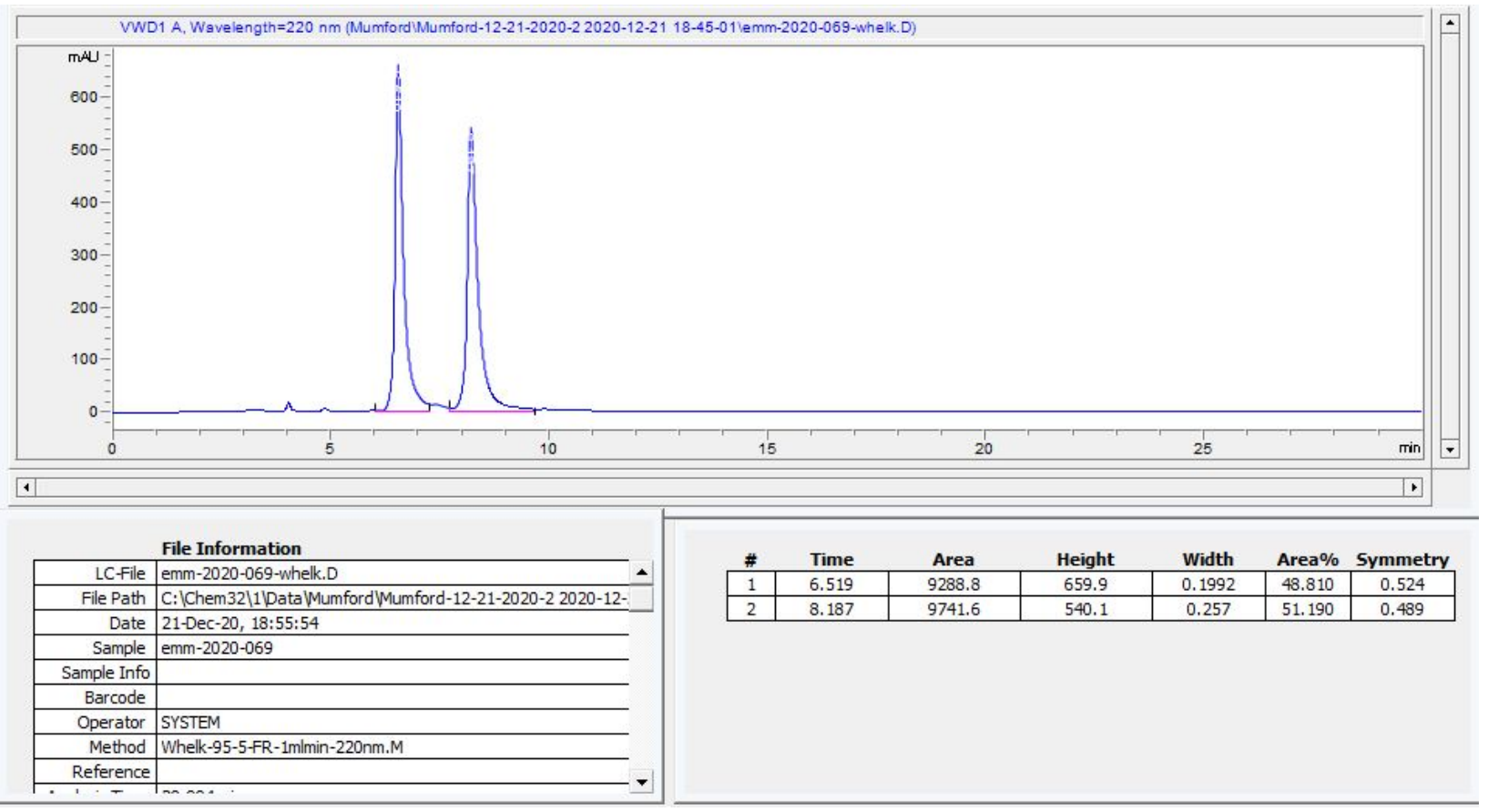

4n- $(R, R)$

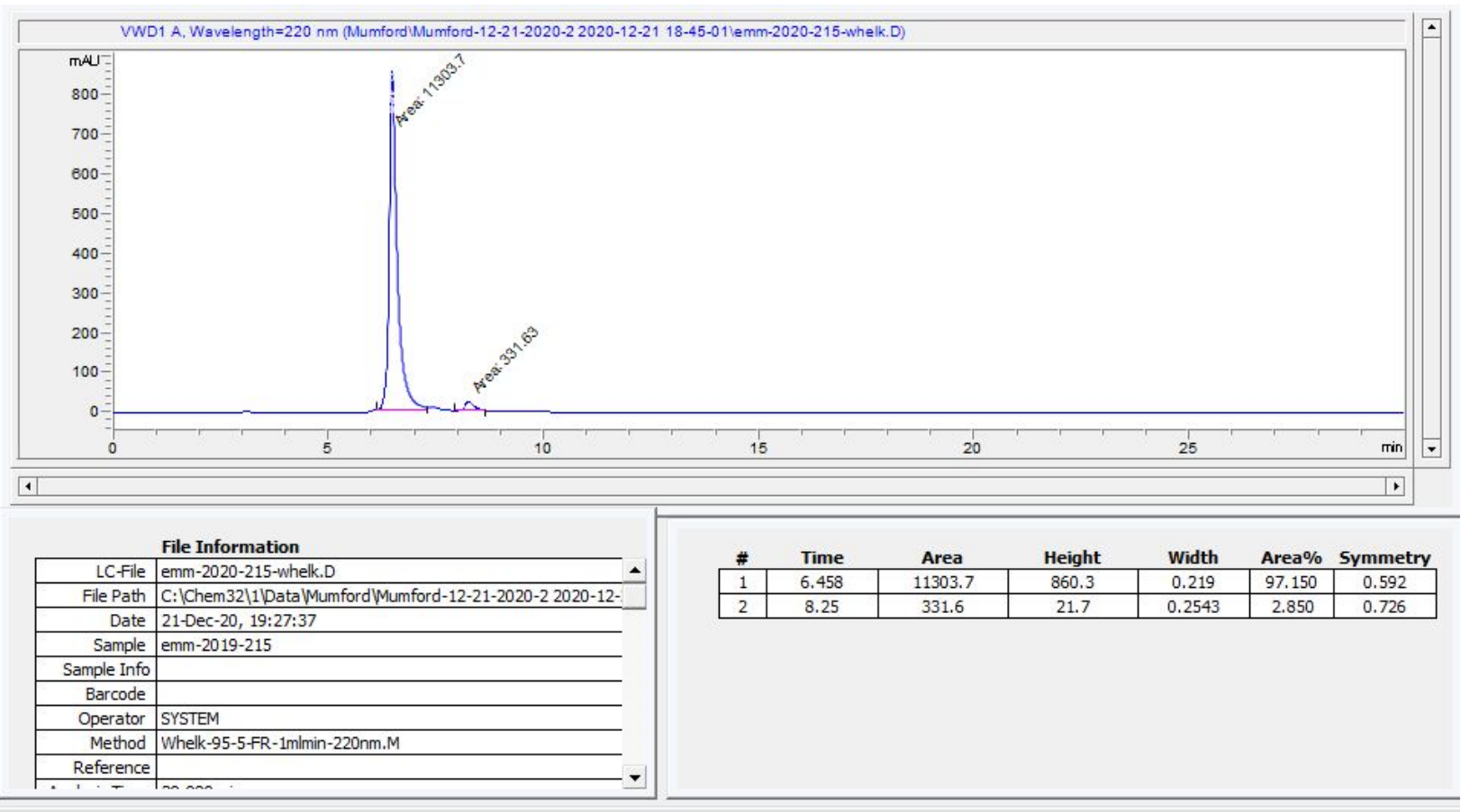


4o ${ }^{1} \mathrm{H}$ NMR $\left(500 \mathrm{MHz}, \mathrm{CDCl}_{3}\right)$

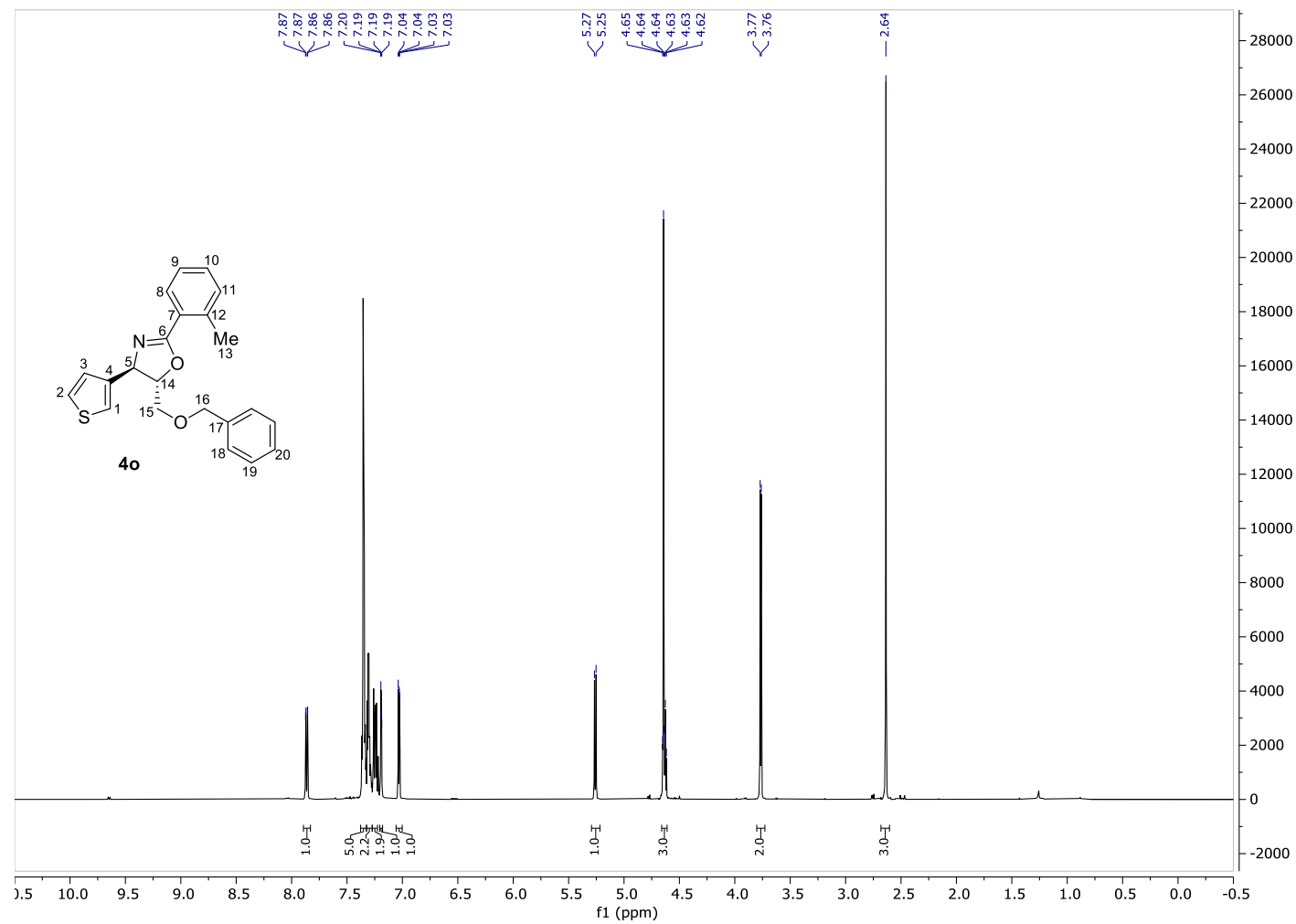

4o ${ }^{13} \mathrm{C}$ NMR (126 MHz, $\mathrm{CDCl}_{3}$ )

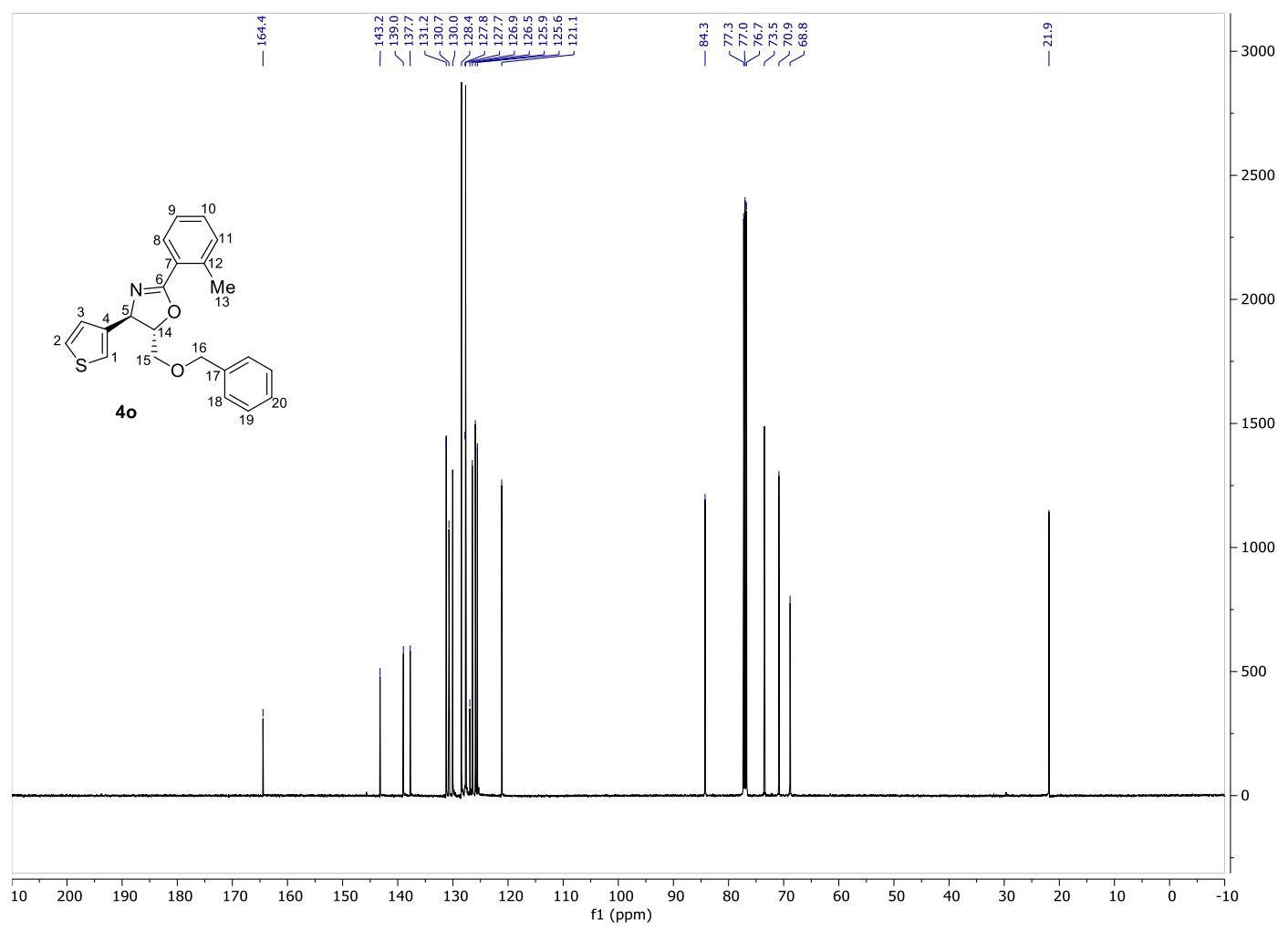


4o-racemic

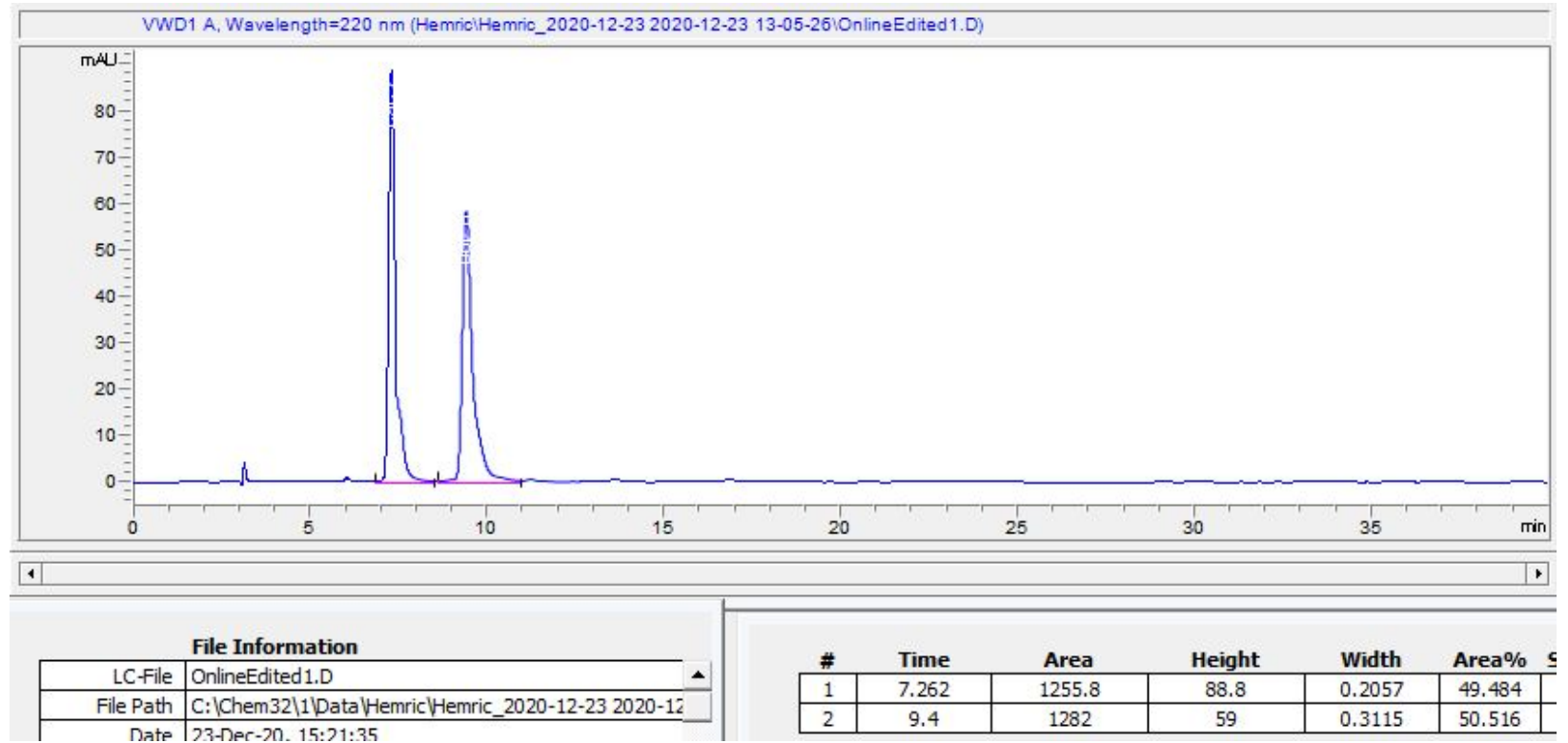

4o- $(R, S)$

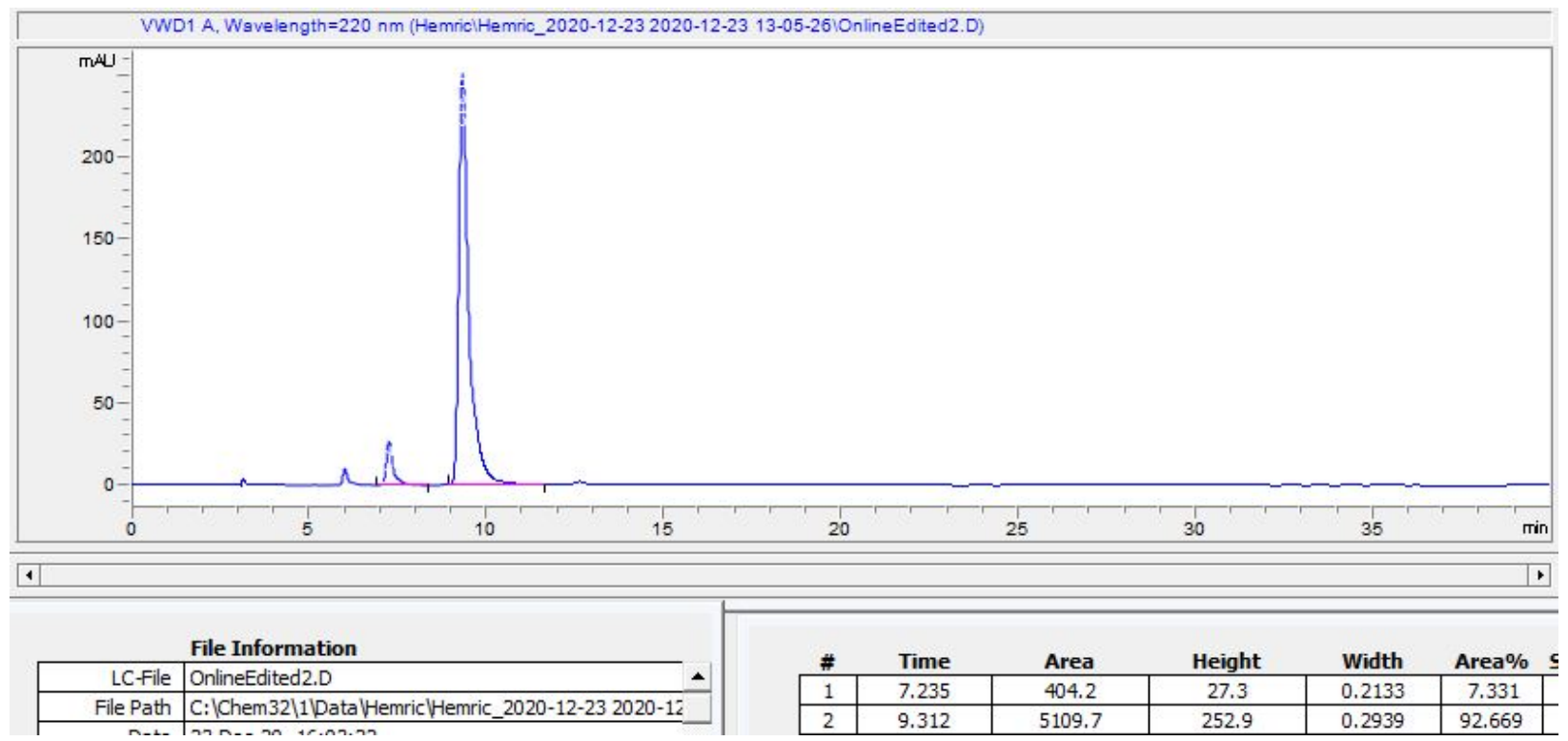


4p ${ }^{1} \mathrm{H}$ NMR (500 MHz, $\mathrm{CDCl}_{3}$ )

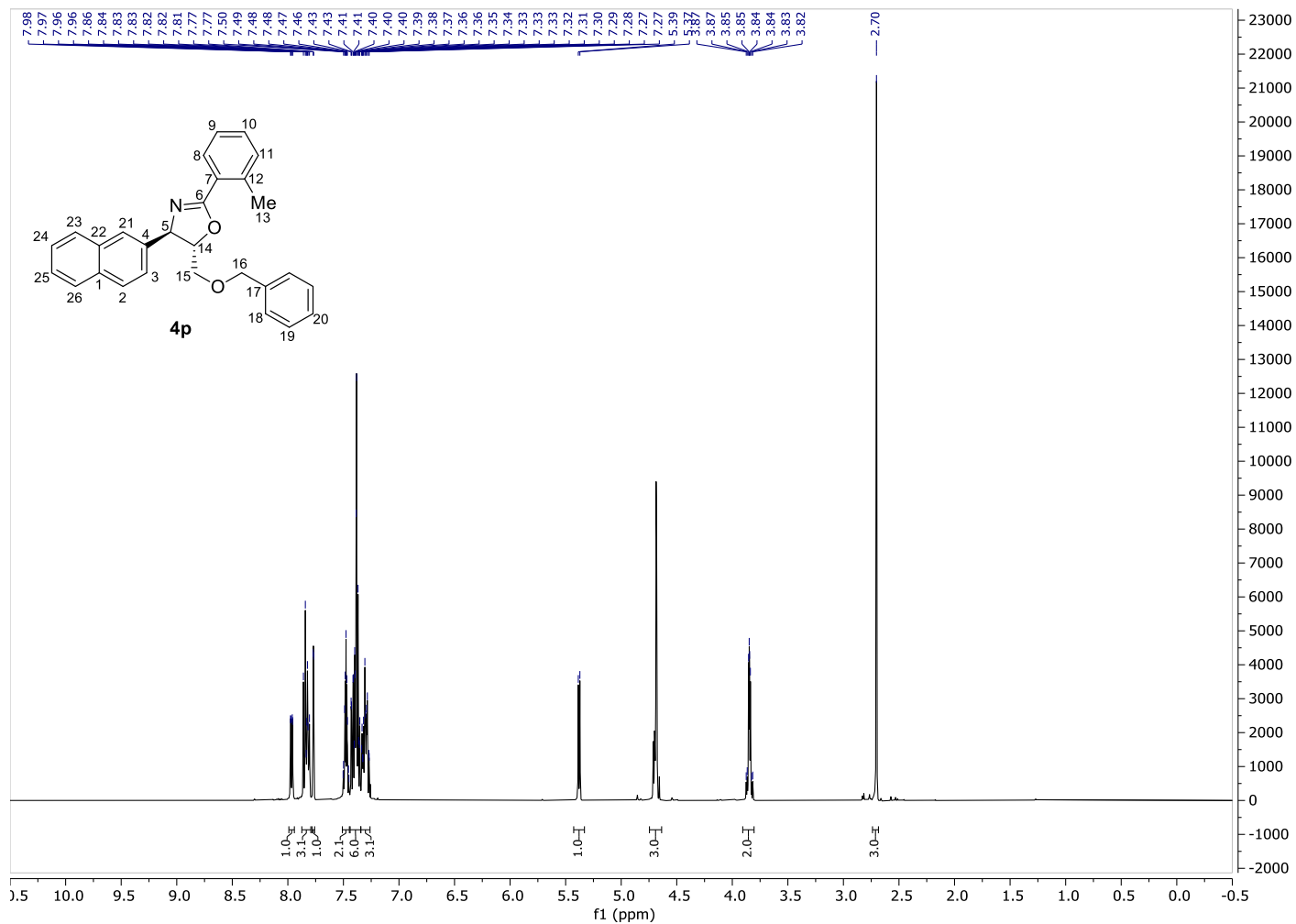

4p ${ }^{13} \mathrm{C}$ NMR (126 MHz, $\left.\mathrm{CDCl}_{3}\right)$

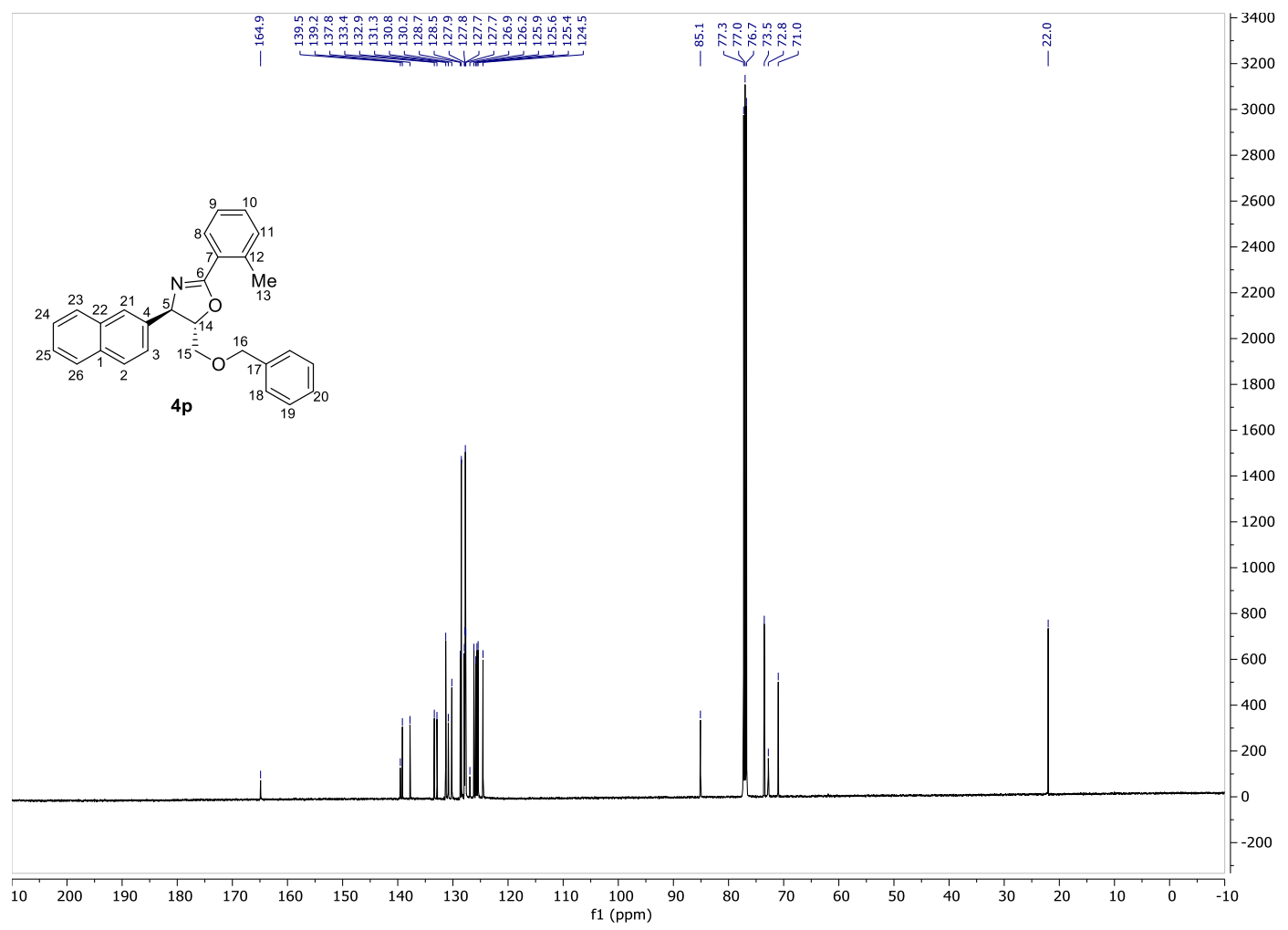




\section{4p-racemic}

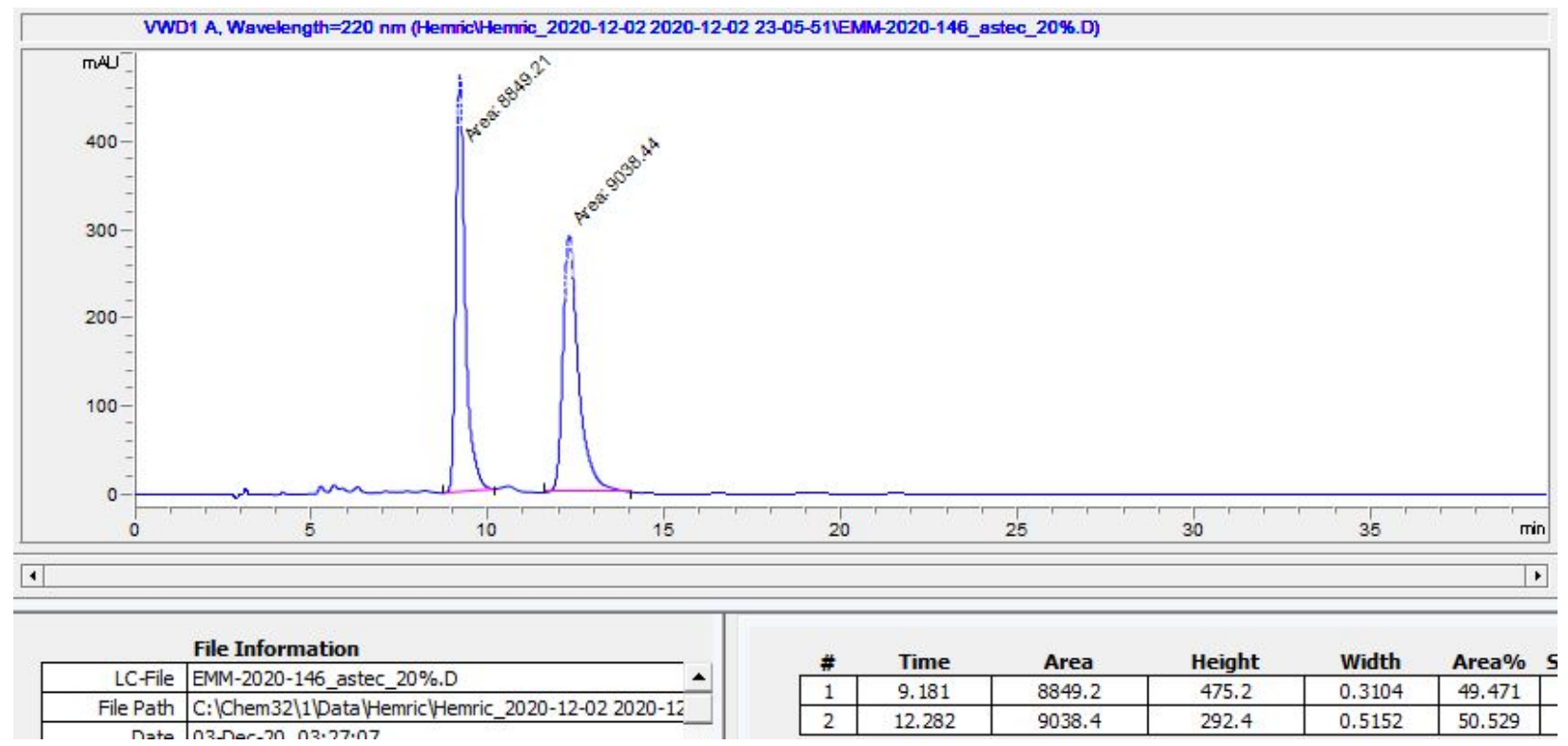

4p- $(R, S)$

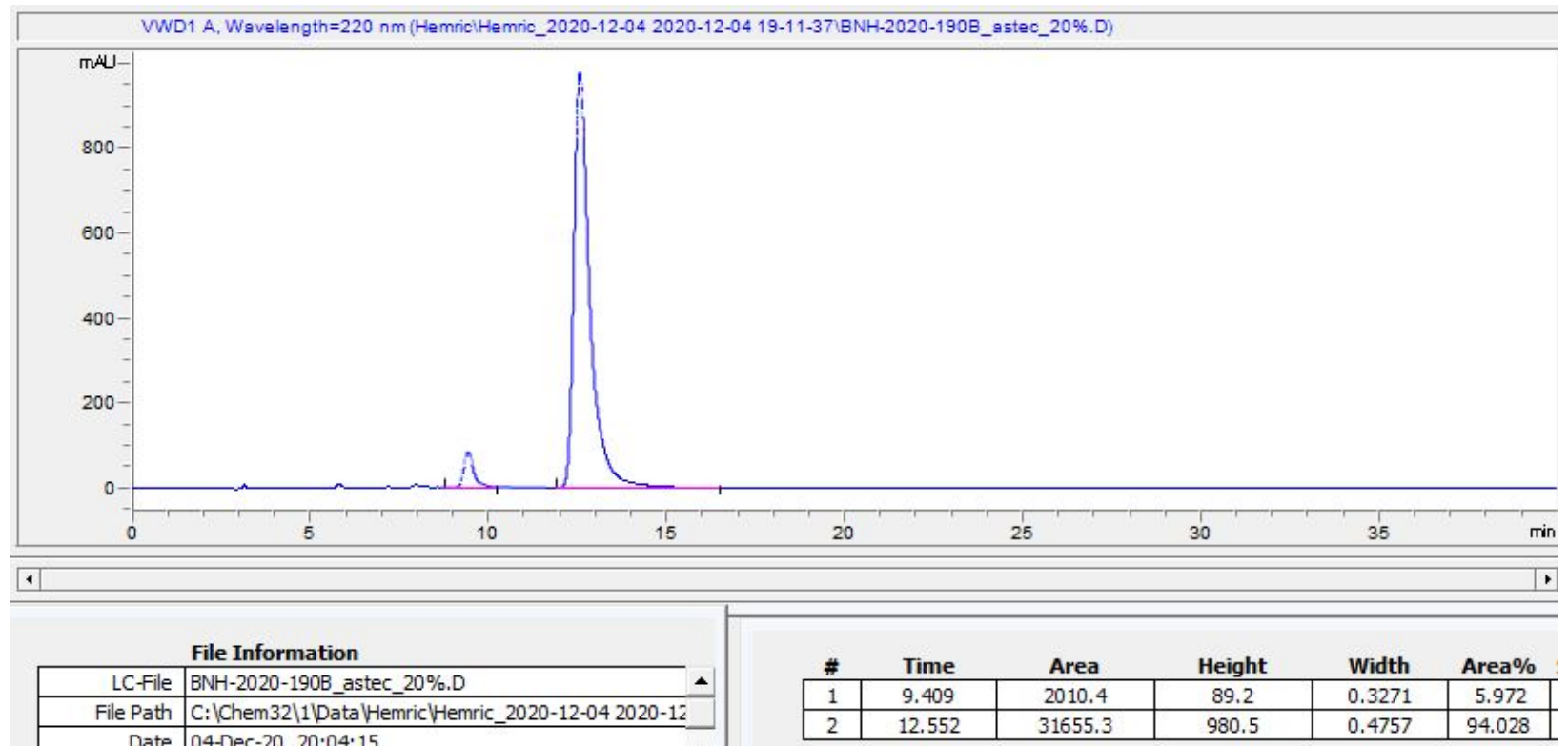


4q ${ }^{1} \mathrm{H}$ NMR (500 MHz, $\mathrm{CDCl}_{3}$ )

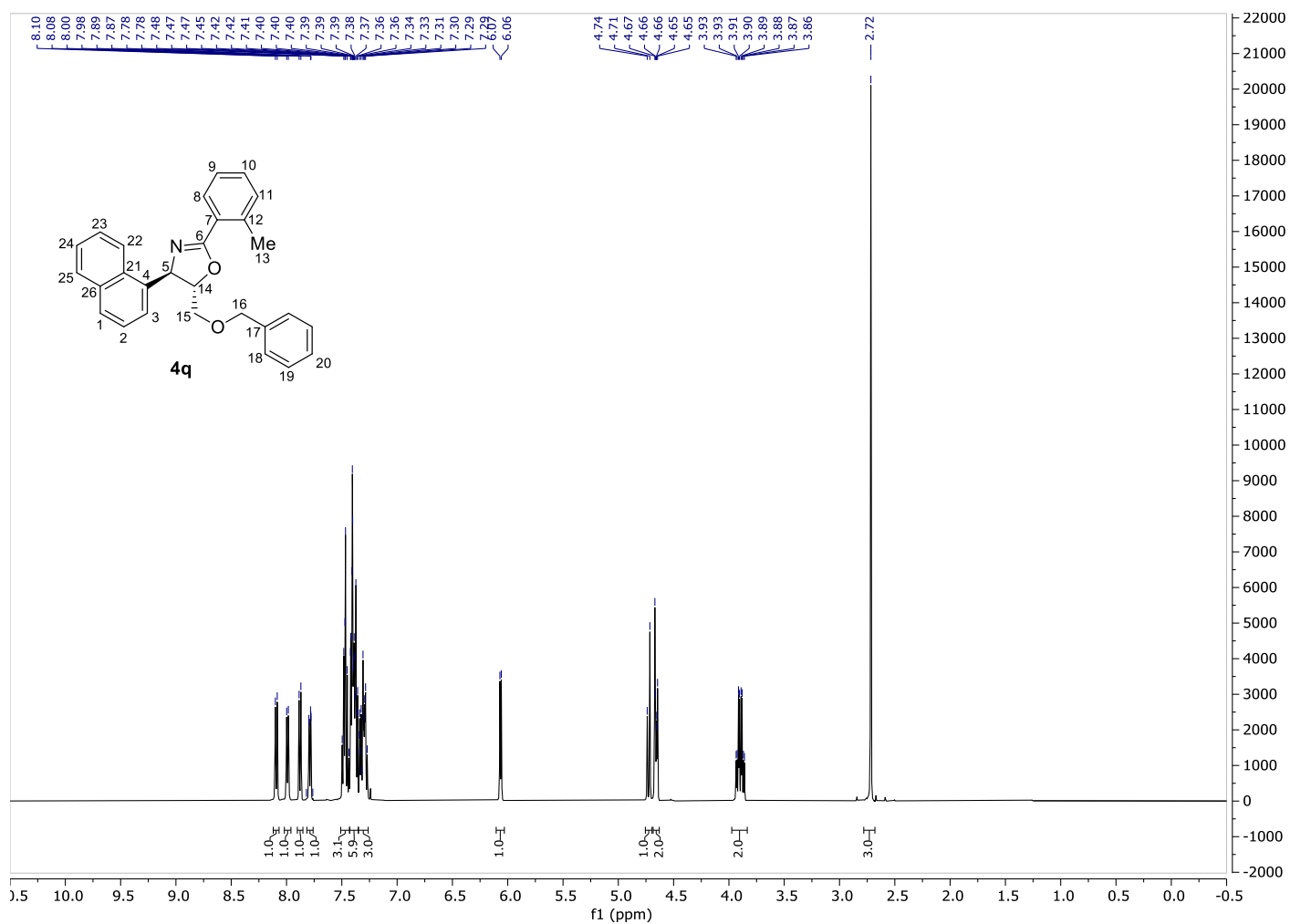

4q ${ }^{13} \mathrm{C}$ NMR (126 MHz, $\left.\mathrm{CDCl}_{3}\right)$

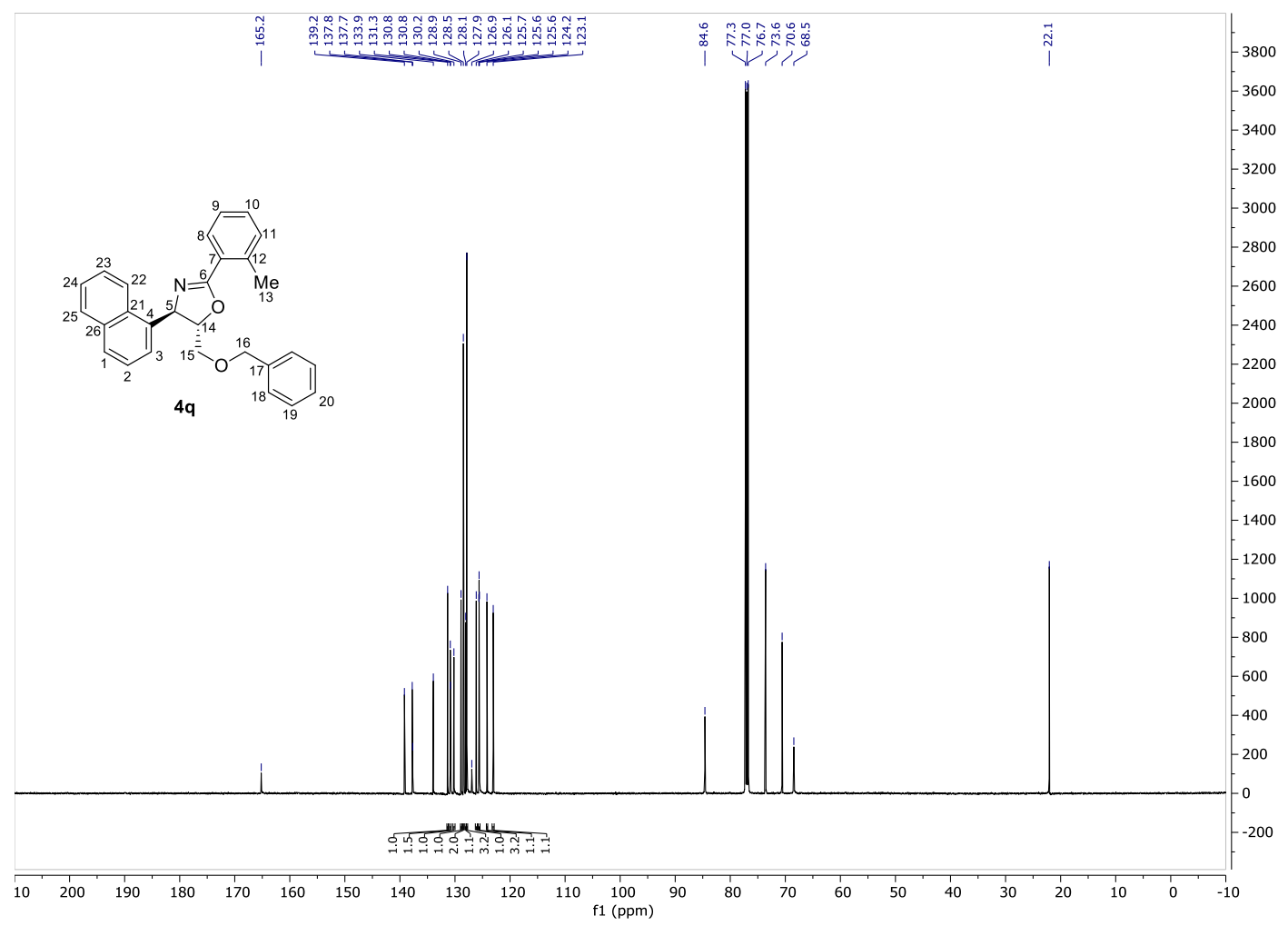




\section{4q-racemic}

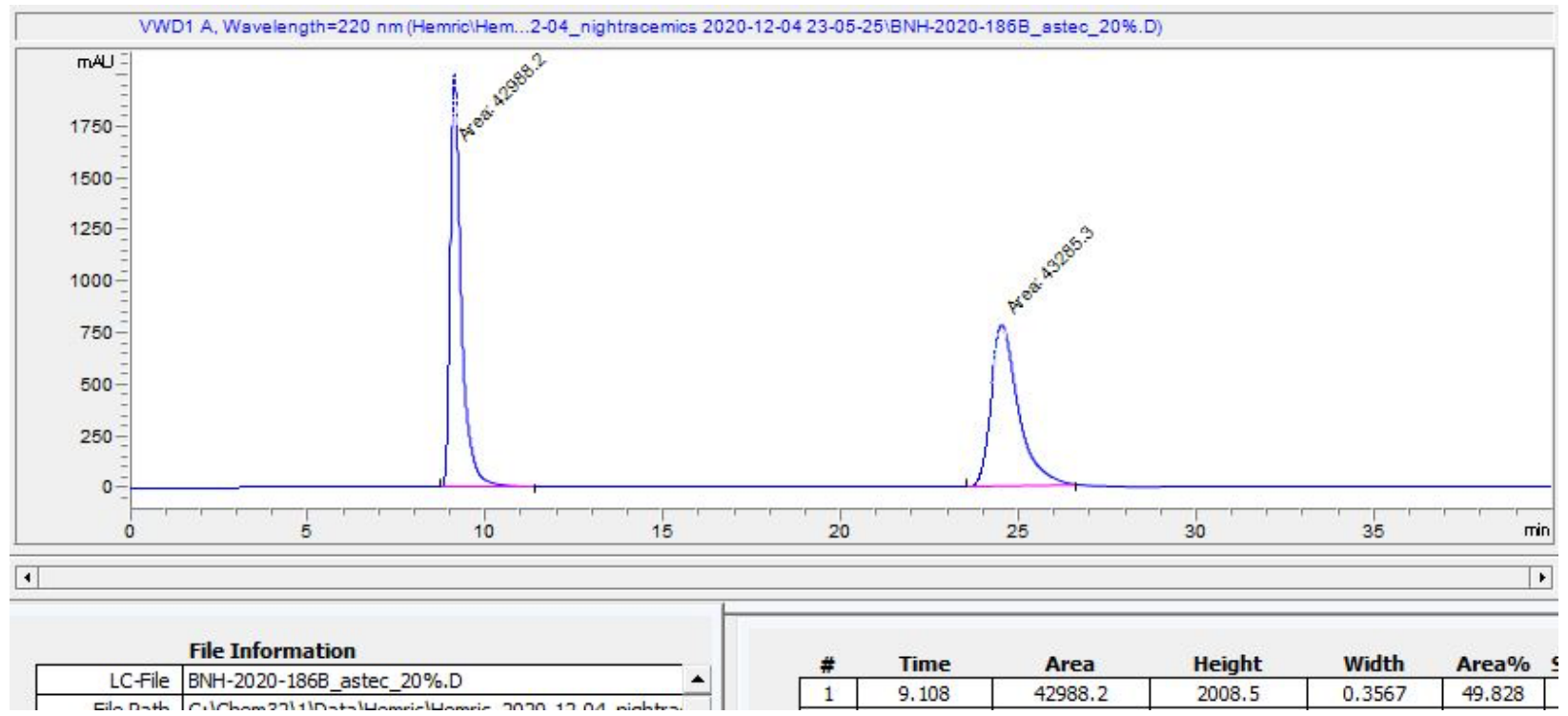

\section{$\mathbf{4 q}-(R, S)$}

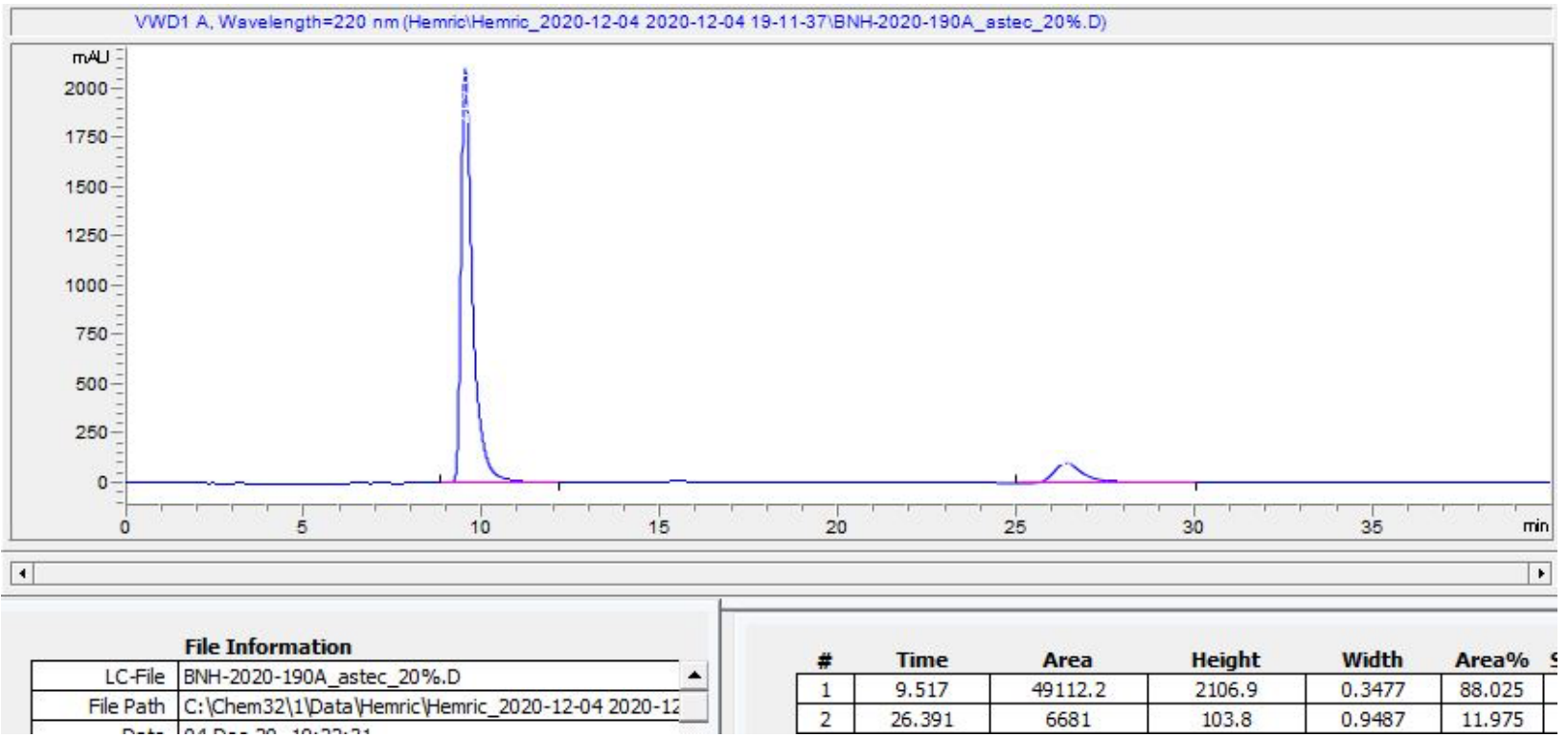




\section{4r ${ }^{1} \mathrm{H}$ NMR (500 MHz, $\left.\mathrm{CDCl}_{3}\right)$}

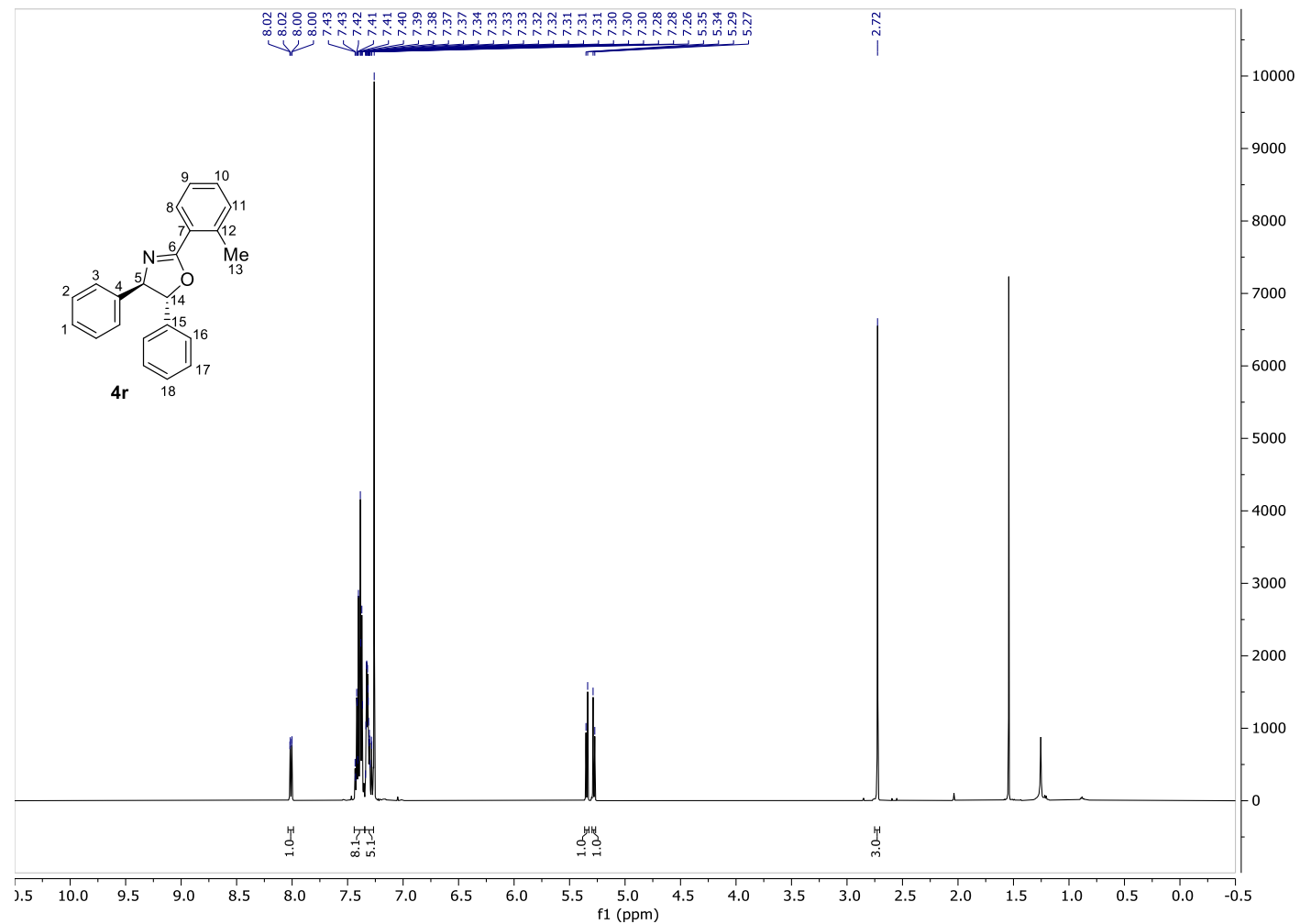

4r ${ }^{13} \mathrm{C}$ NMR (126 MHz, $\left.\mathrm{CDCl}_{3}\right)$

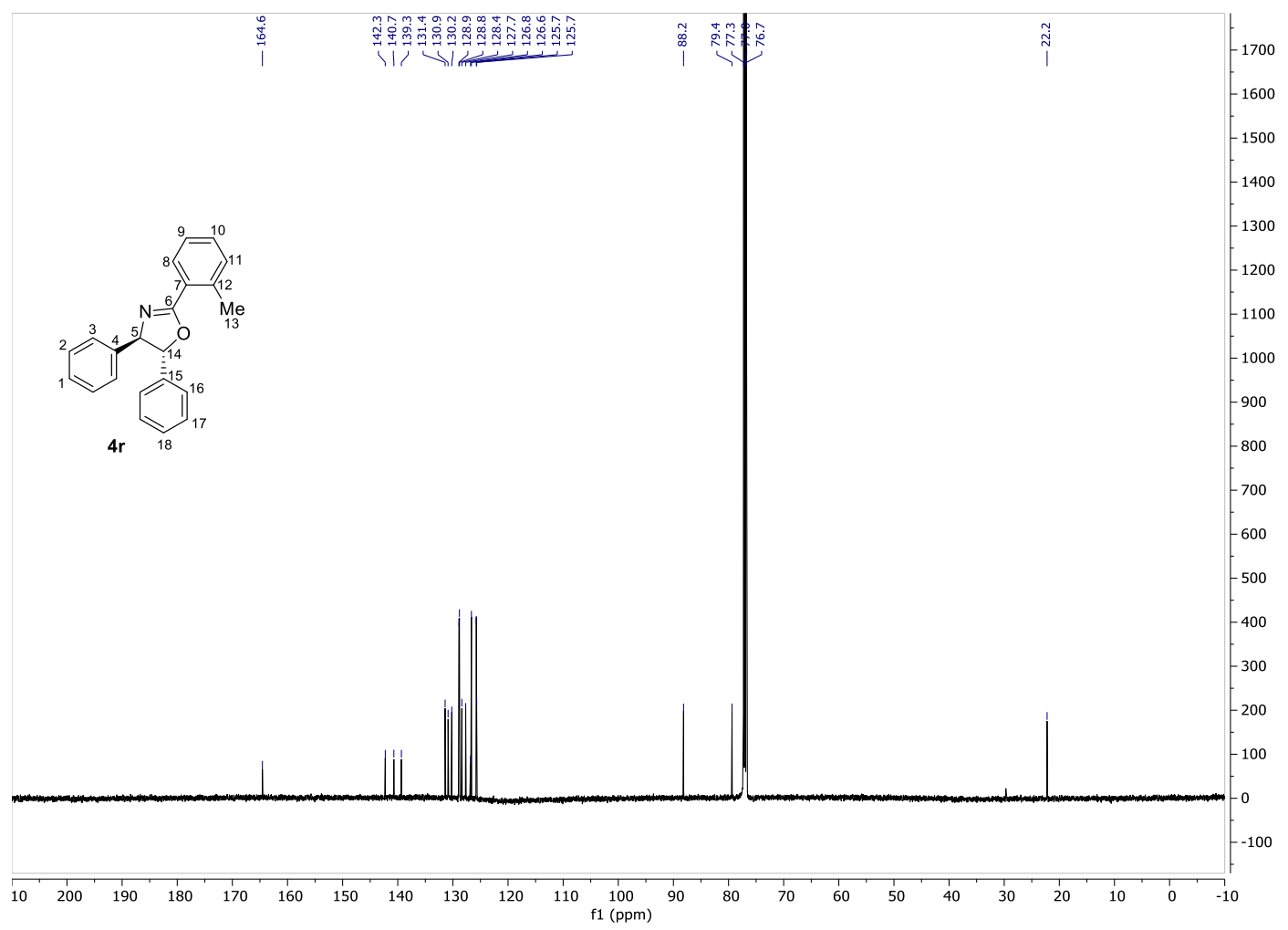




\section{4r-racemic}

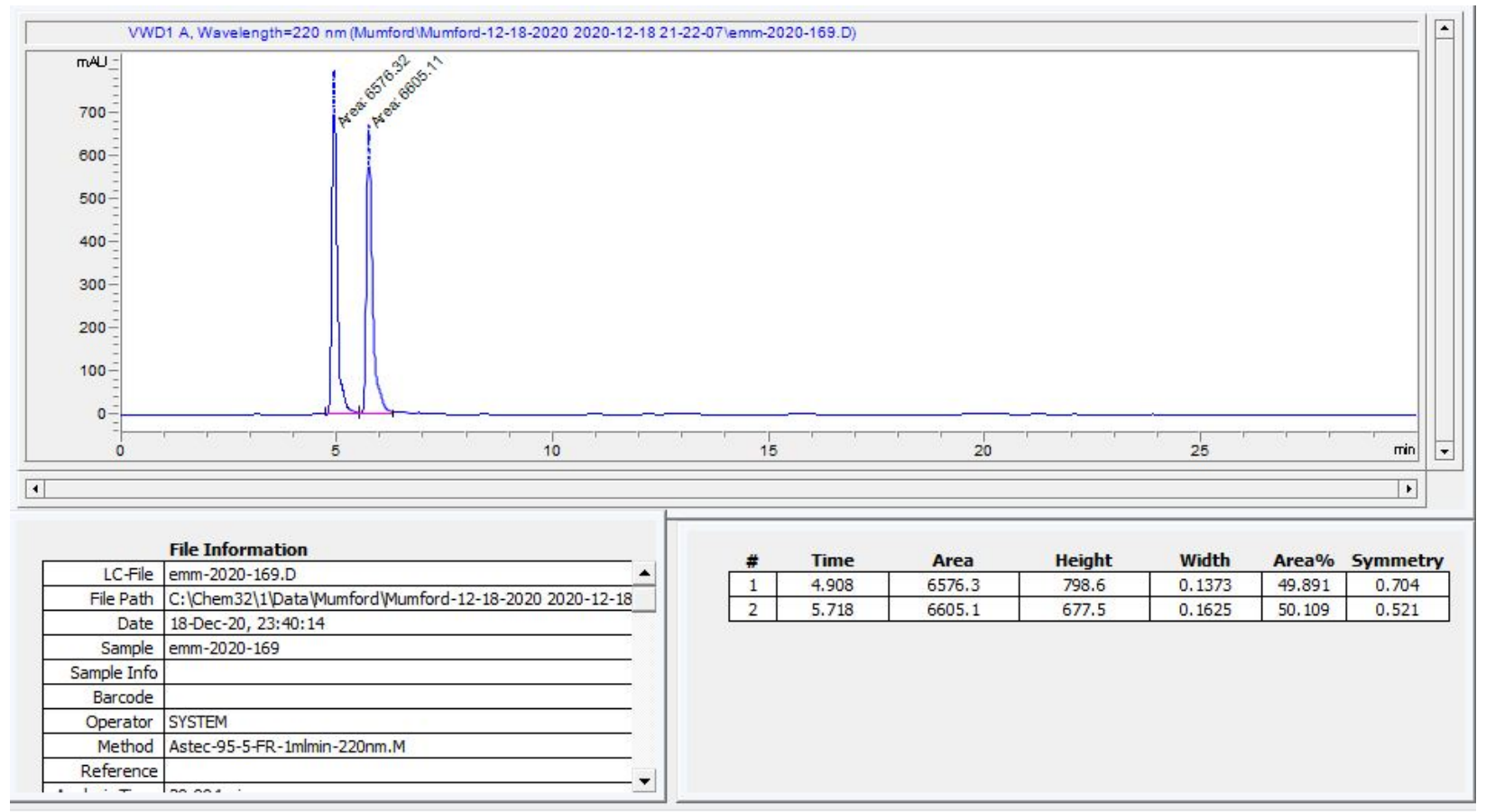

\section{$4 \mathbf{r}-(R, R)$}

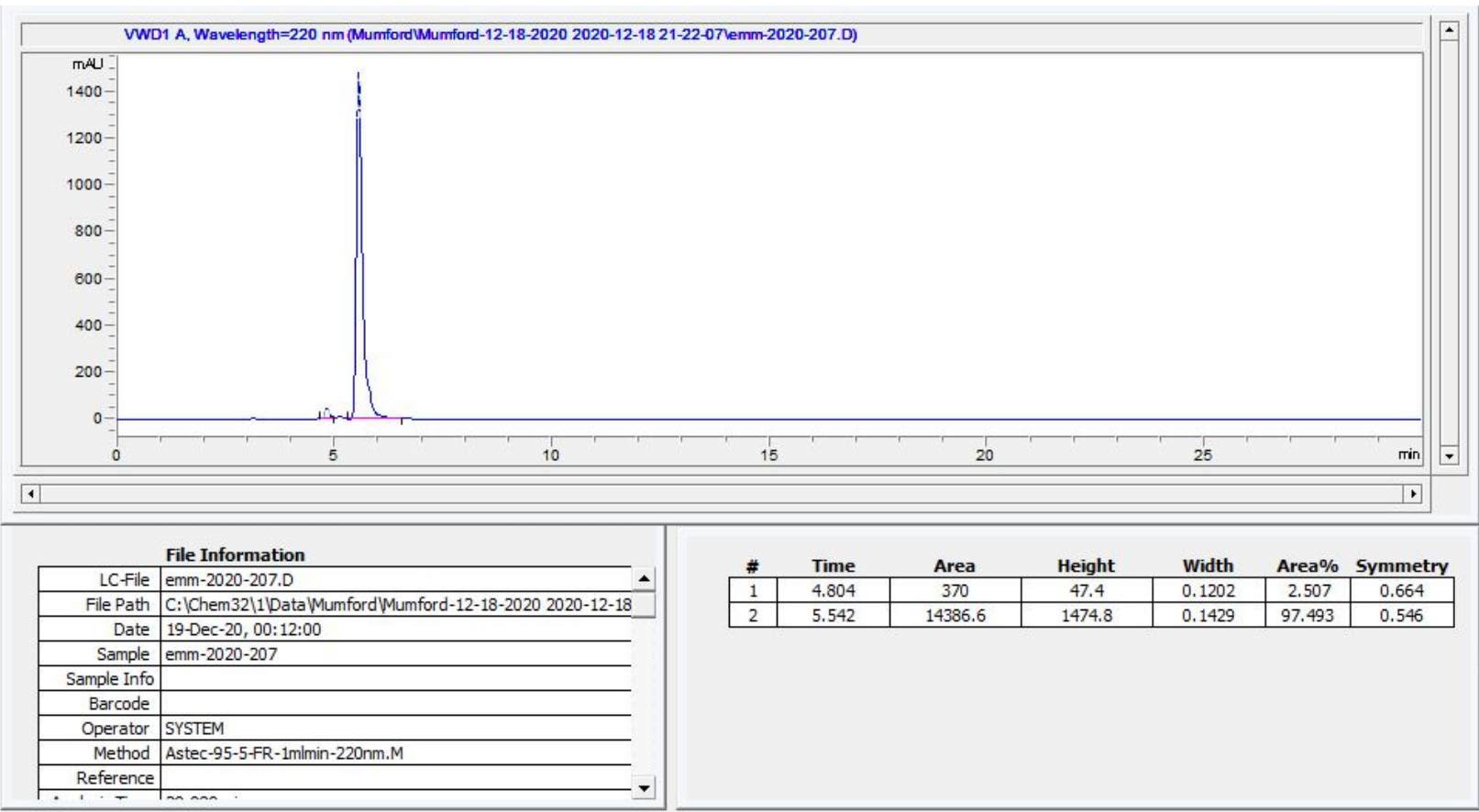


4s ${ }^{1} \mathrm{H}$ NMR (500 MHz, $\mathrm{CDCl}_{3}$ )

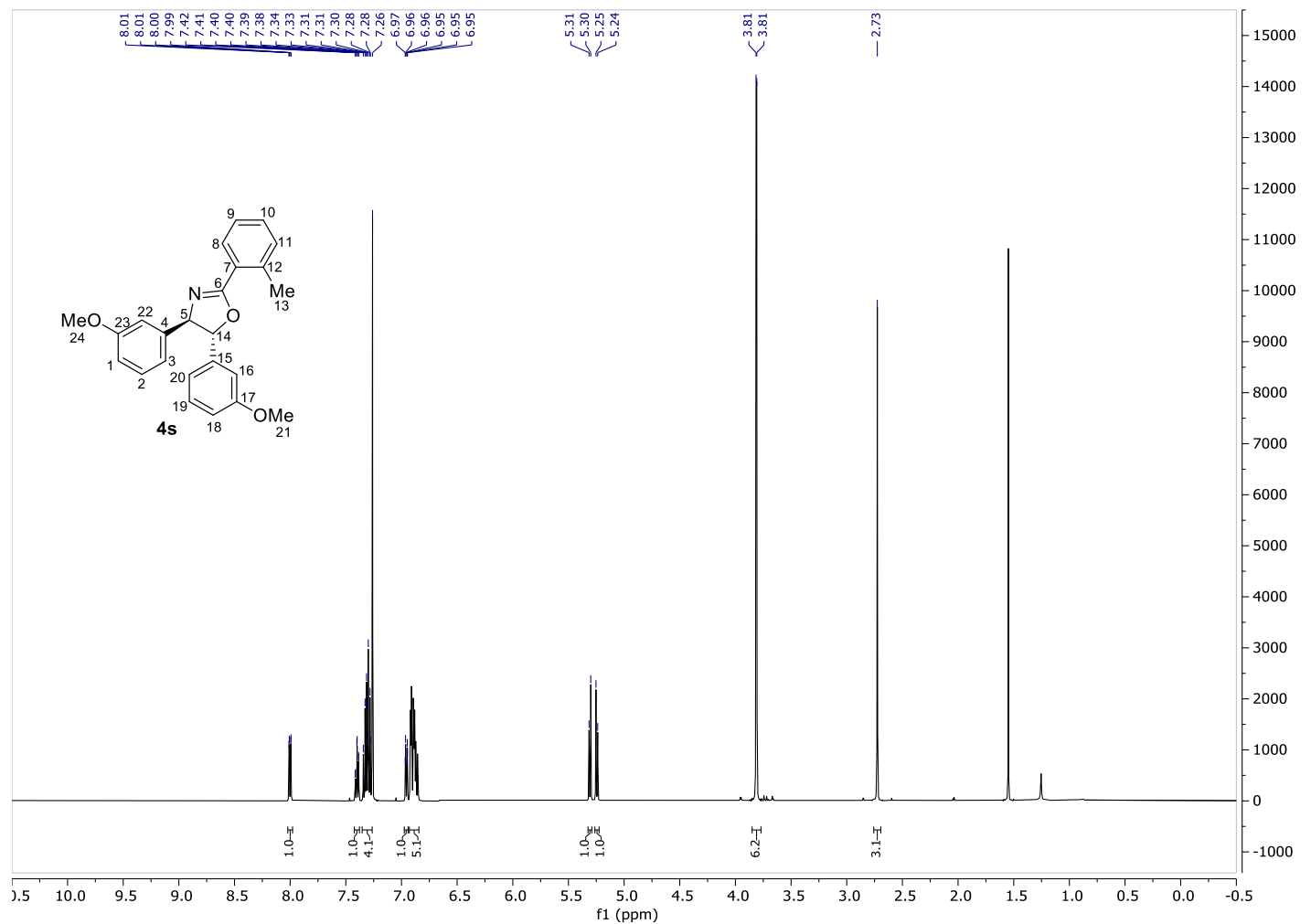

4s ${ }^{13} \mathrm{C}$ NMR (126 MHz, $\left.\mathrm{CDCl}_{3}\right)$

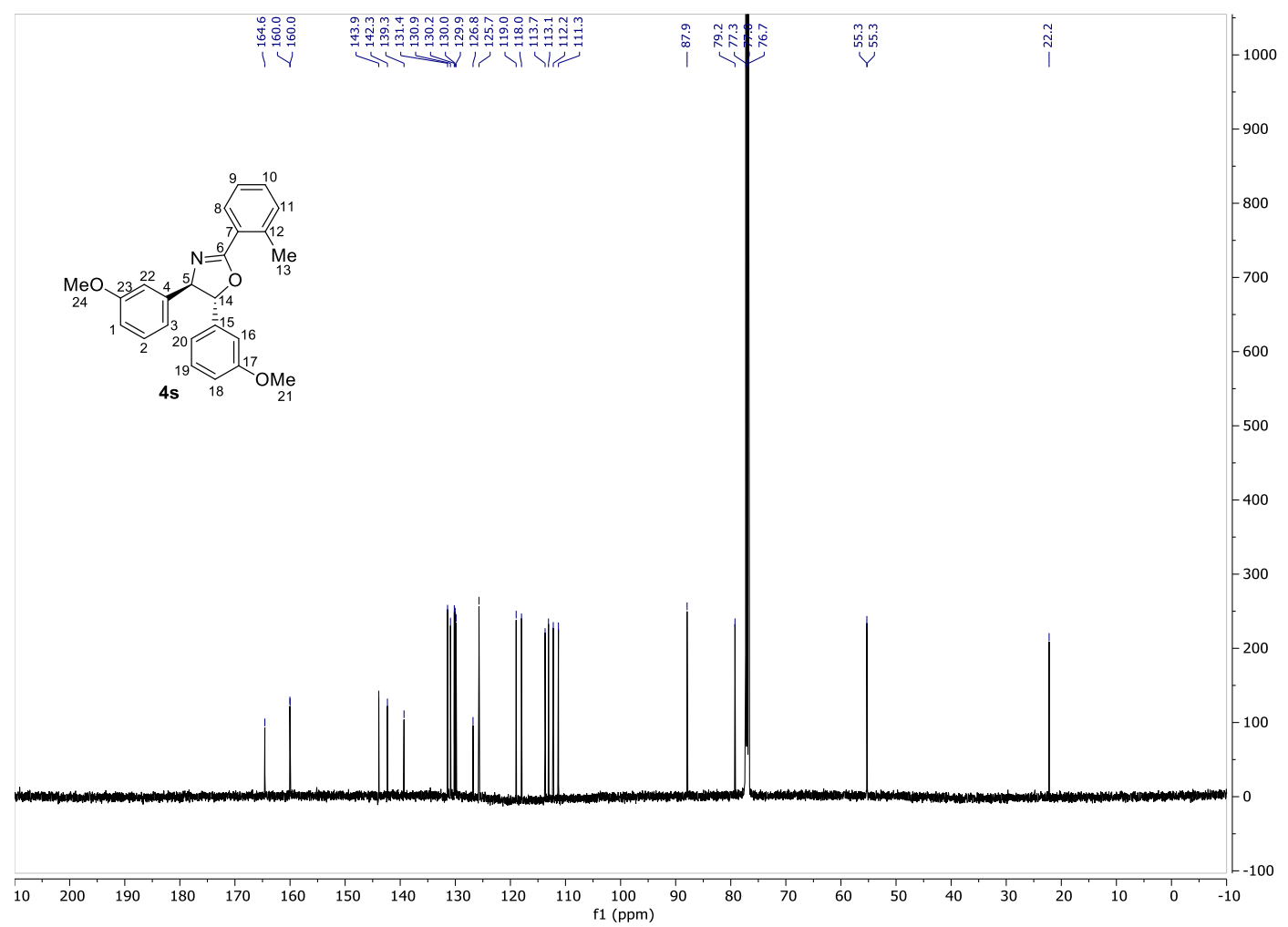




\section{4s-racemic}

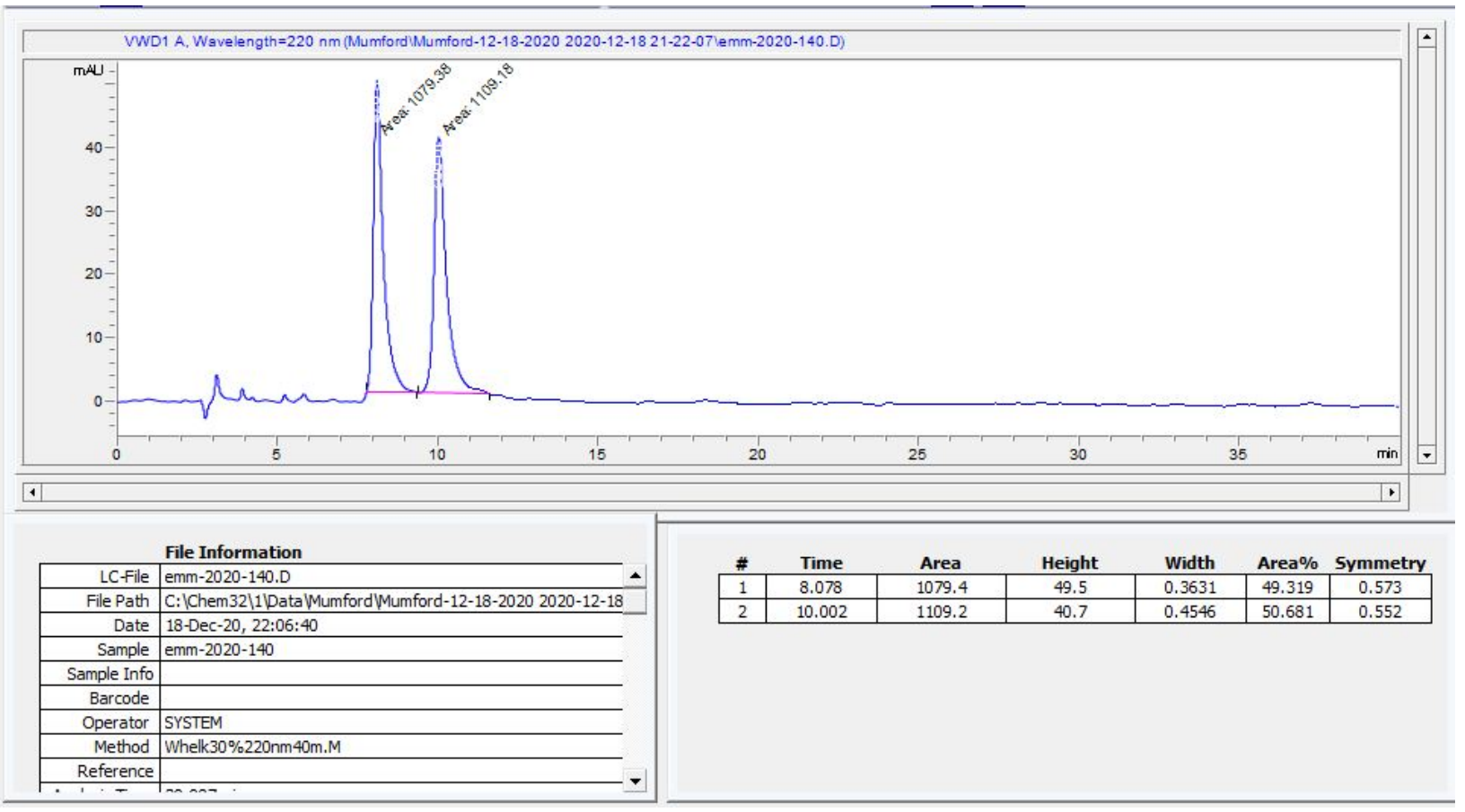

\section{4s- $(R, R)$}

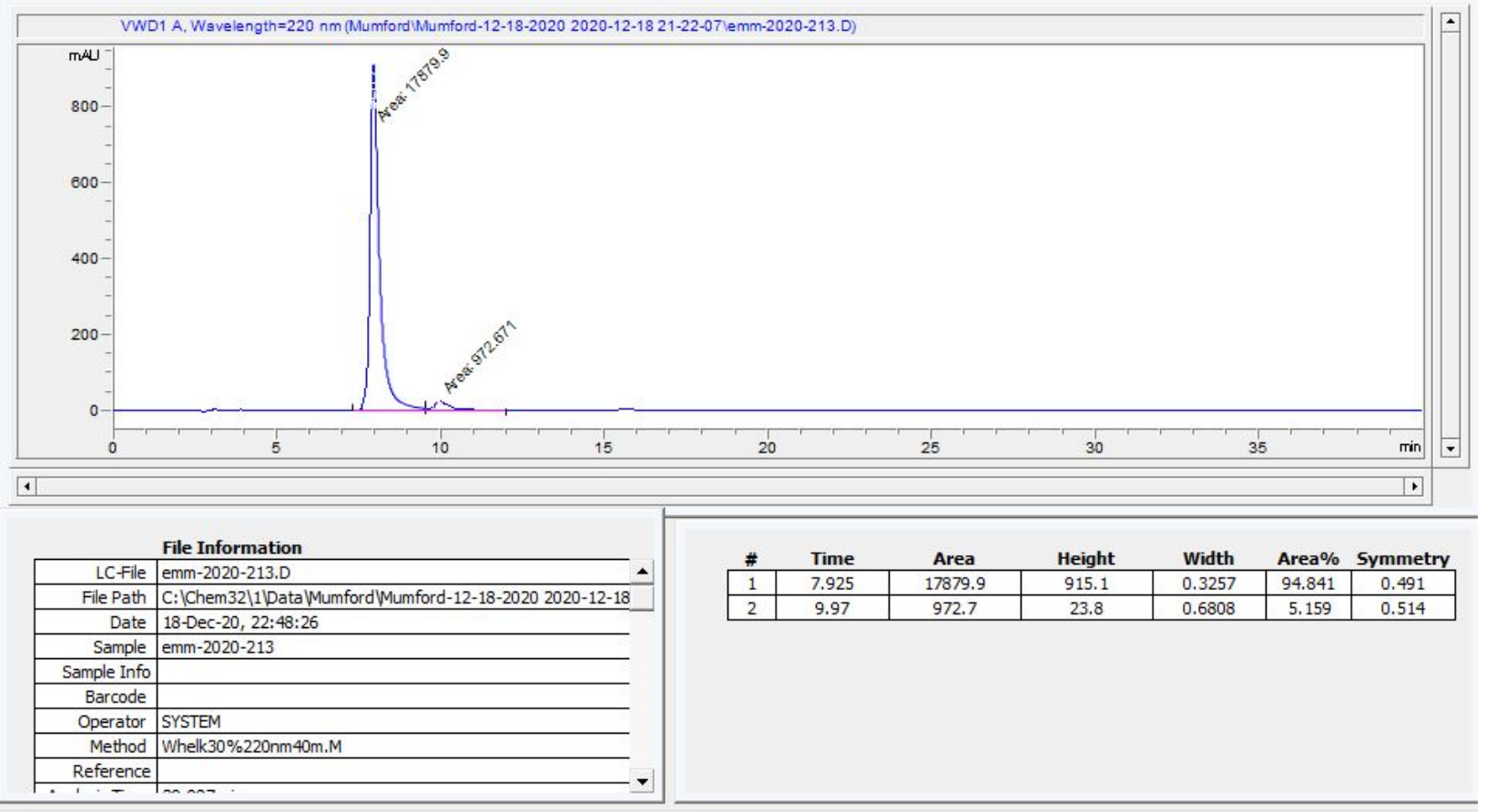


4t ${ }^{1} \mathrm{H}$ NMR (500 MHz, $\left.\mathrm{CDCl}_{3}\right)$

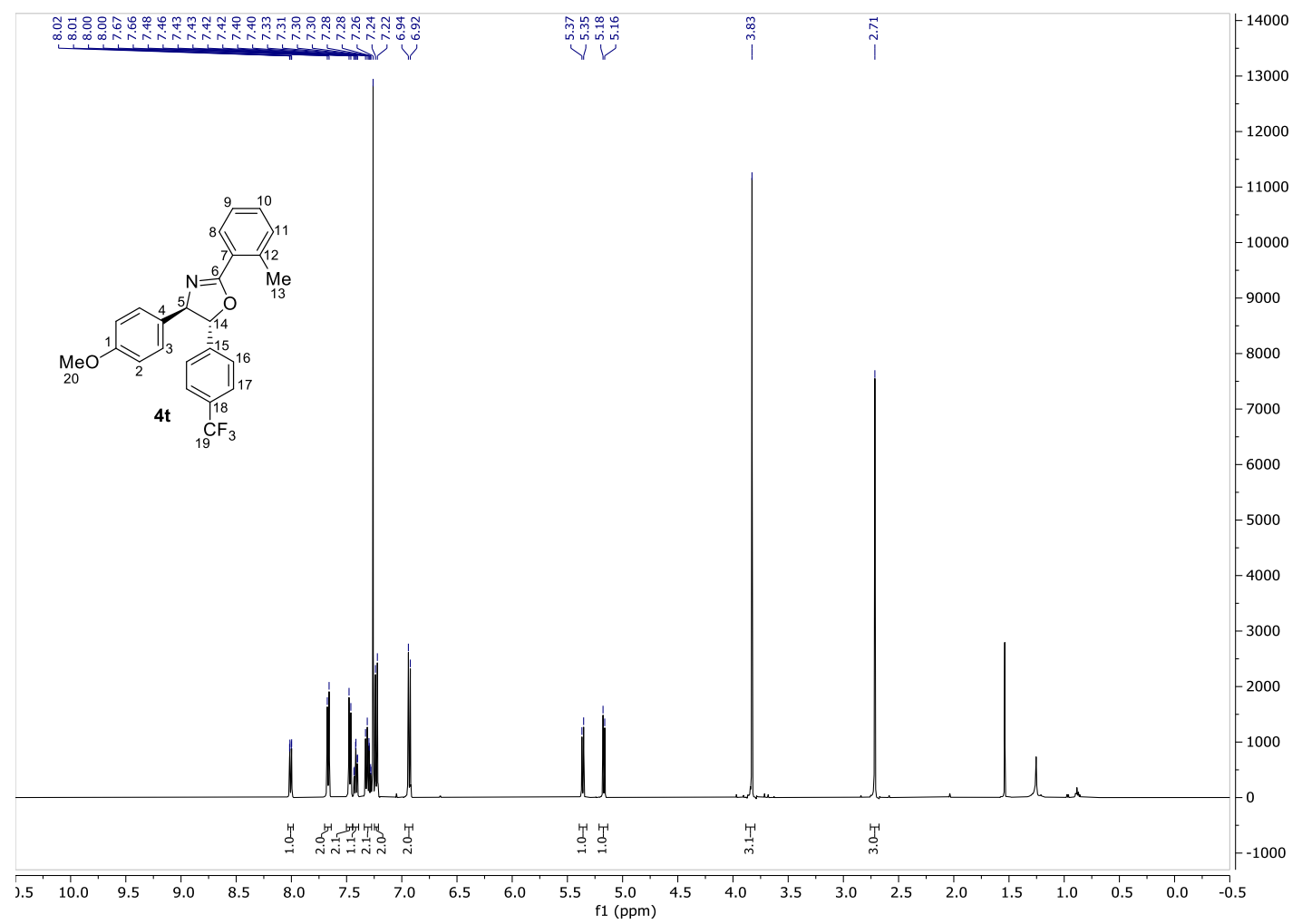

4t ${ }^{13} \mathrm{C}$ NMR (126 MHz, $\mathrm{CDCl}_{3}$ )

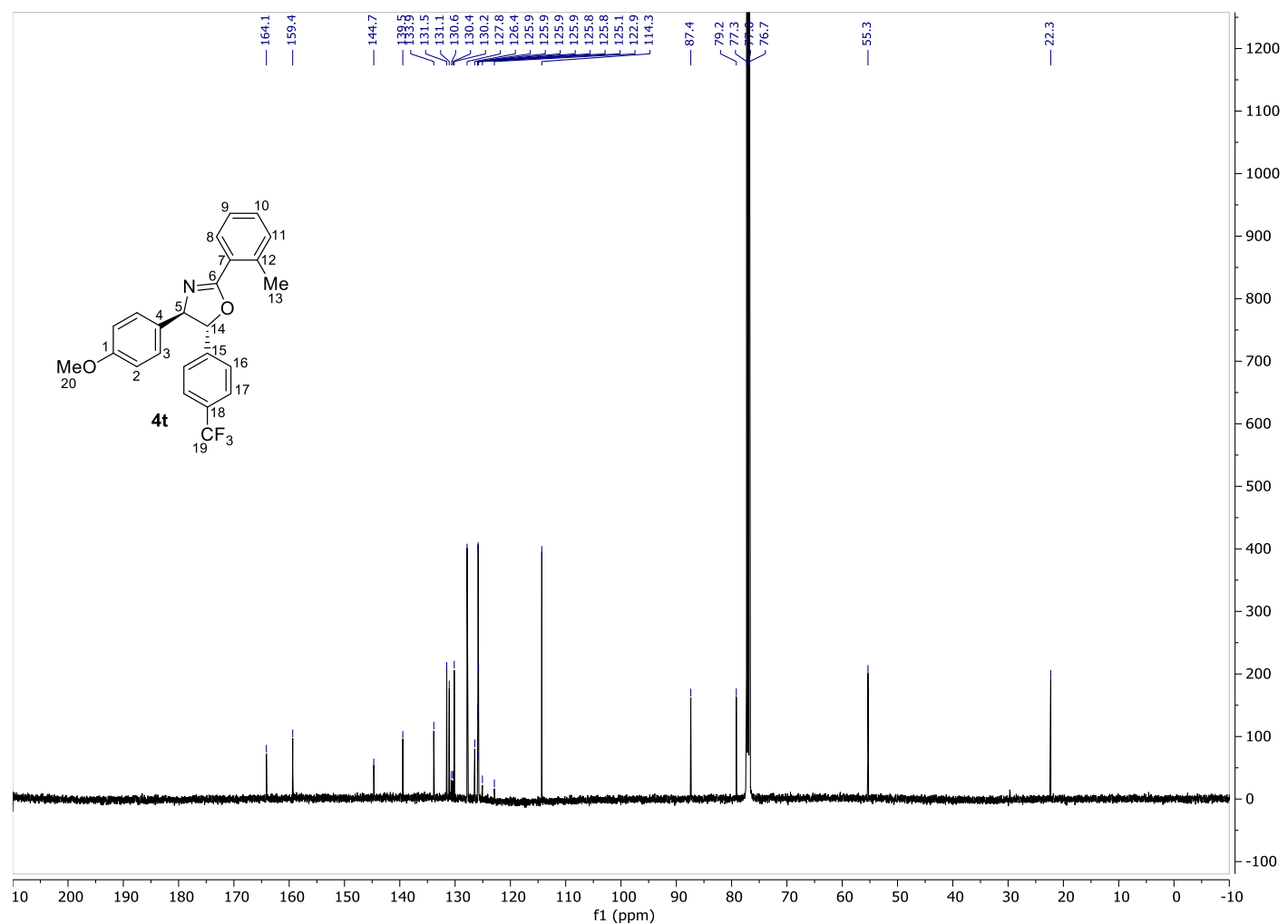


4t ${ }^{19} \mathrm{~F}$ NMR (471 MHz, $\mathrm{CDCl}_{3}$ with $\mathrm{C}_{6} \mathrm{~F}_{5}$ as standard)

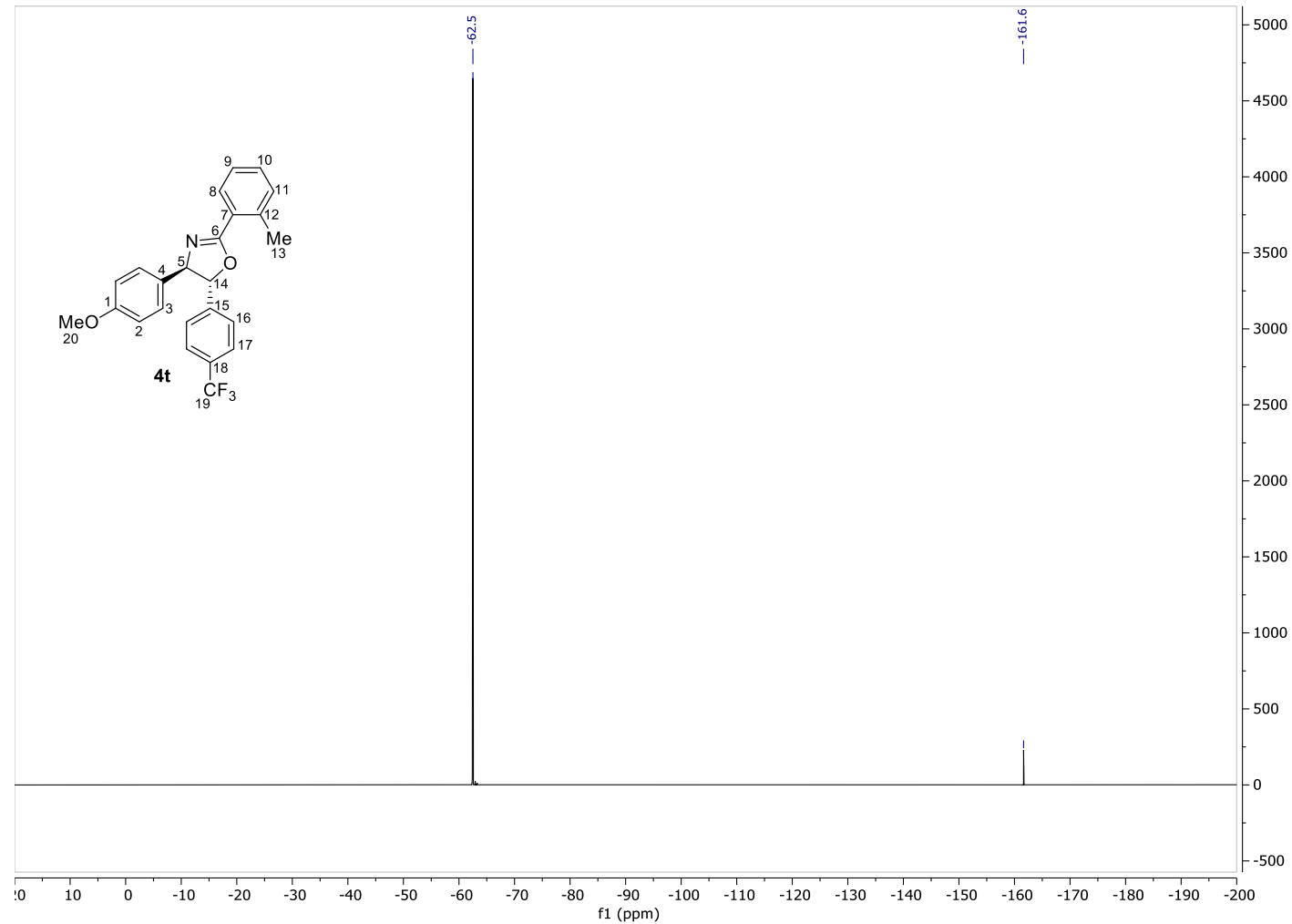


4t-racemic

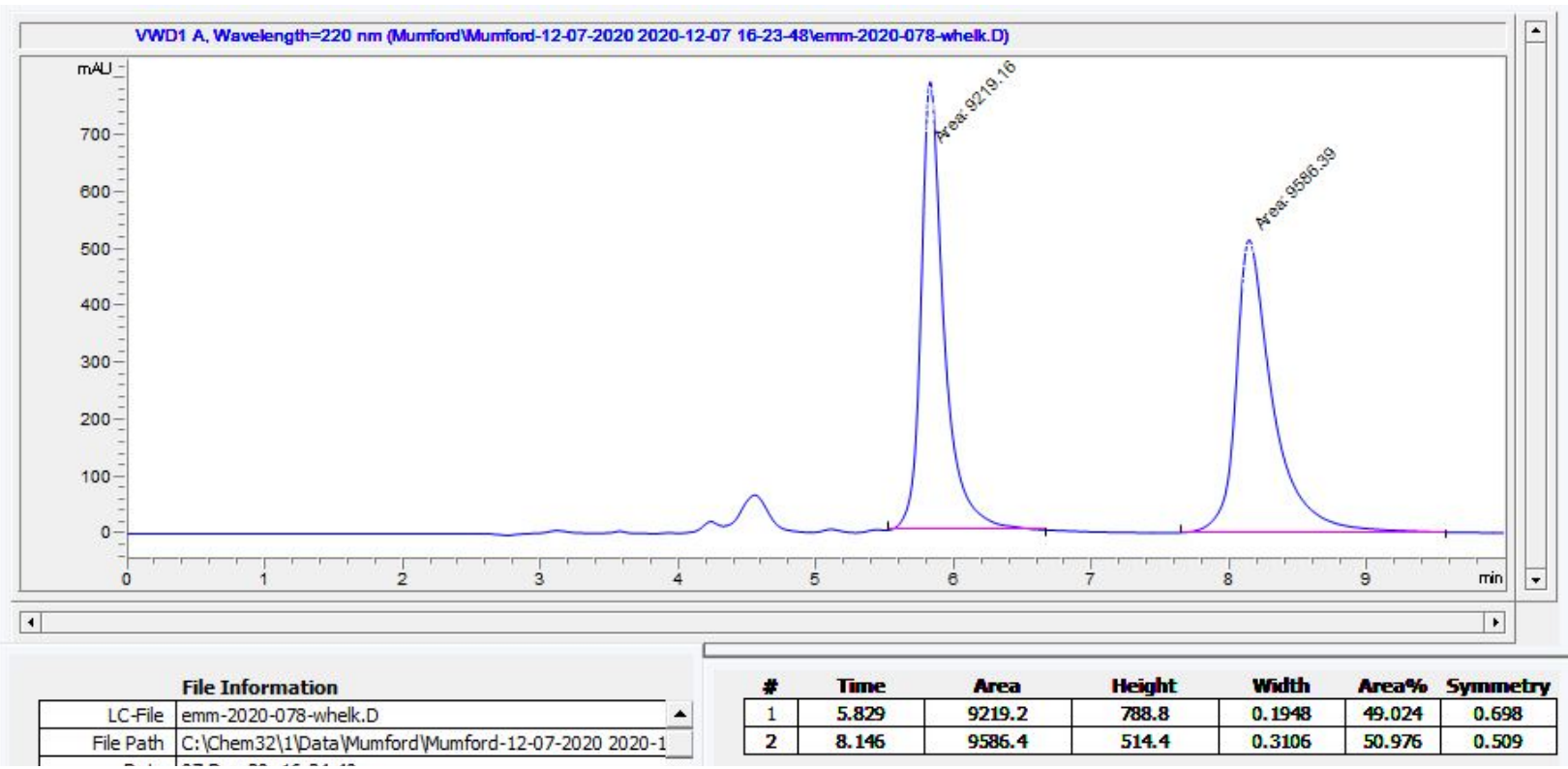

\section{4t- $(R, R)$}

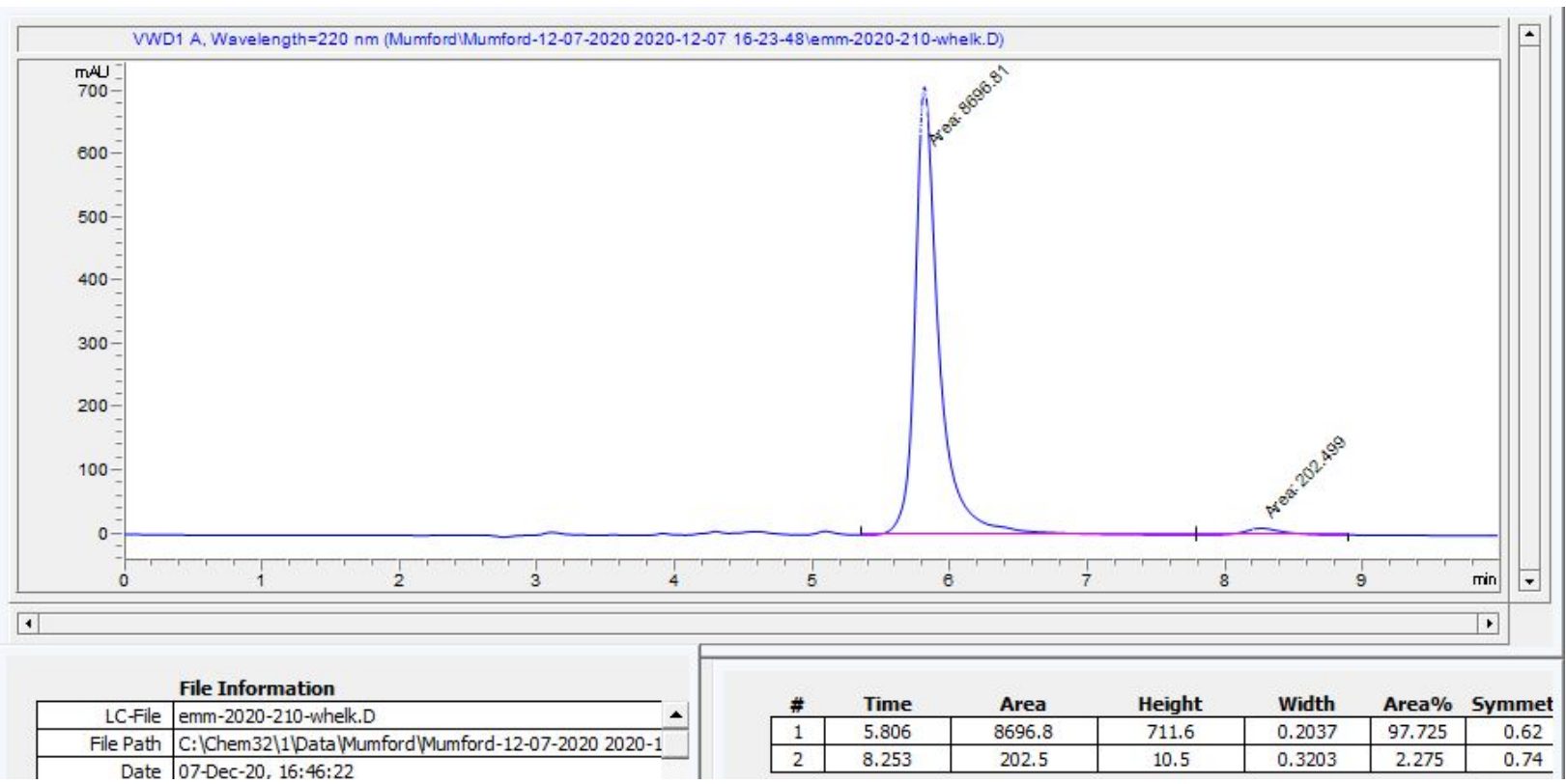


$5{ }^{1} \mathrm{H}$ NMR (500 MHz, $\left.\mathrm{CDCl}_{3}\right)$

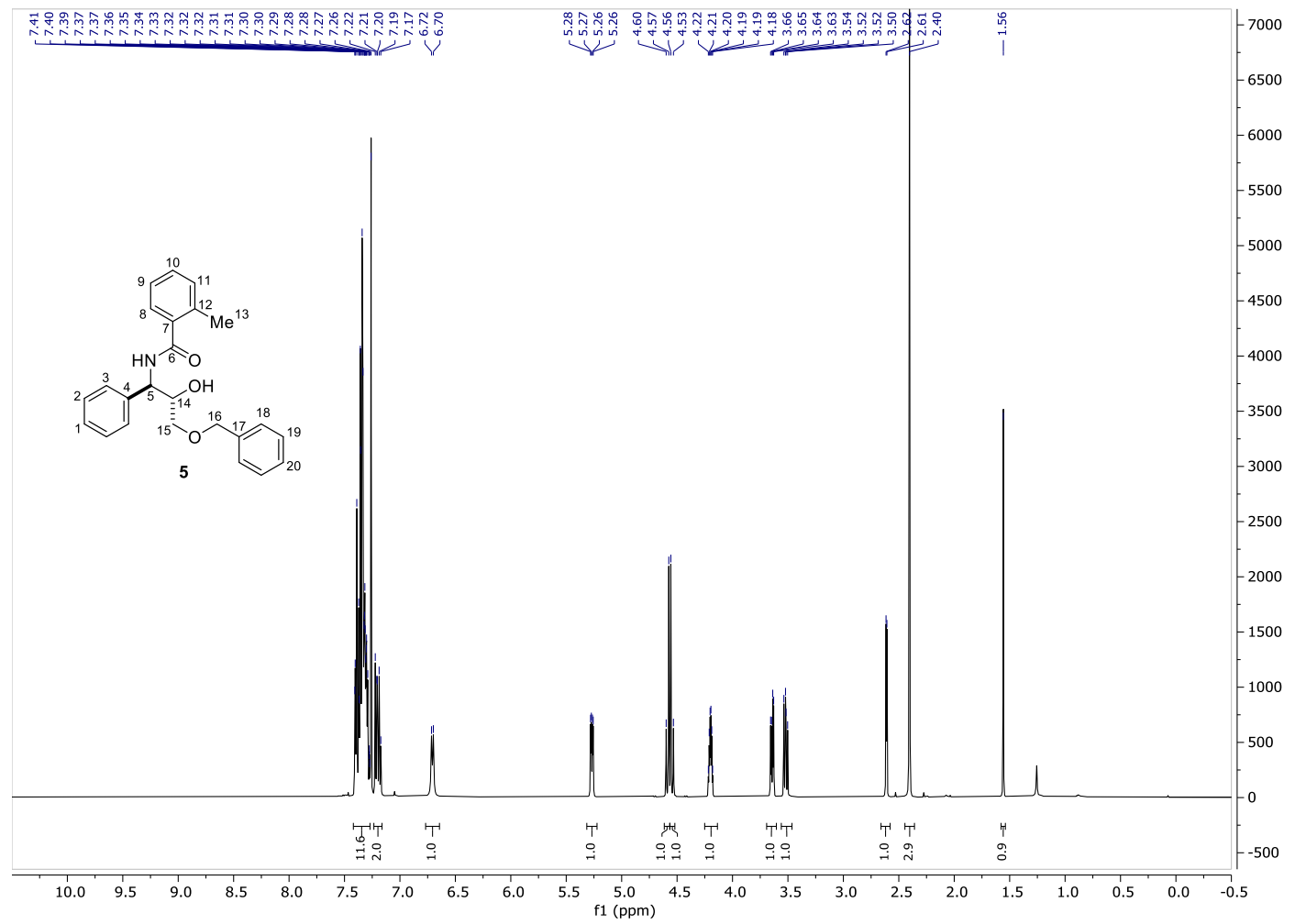

$5{ }^{13} \mathrm{C} \mathrm{NMR}\left(126 \mathrm{MHz}, \mathrm{CDCl}_{3}\right)$

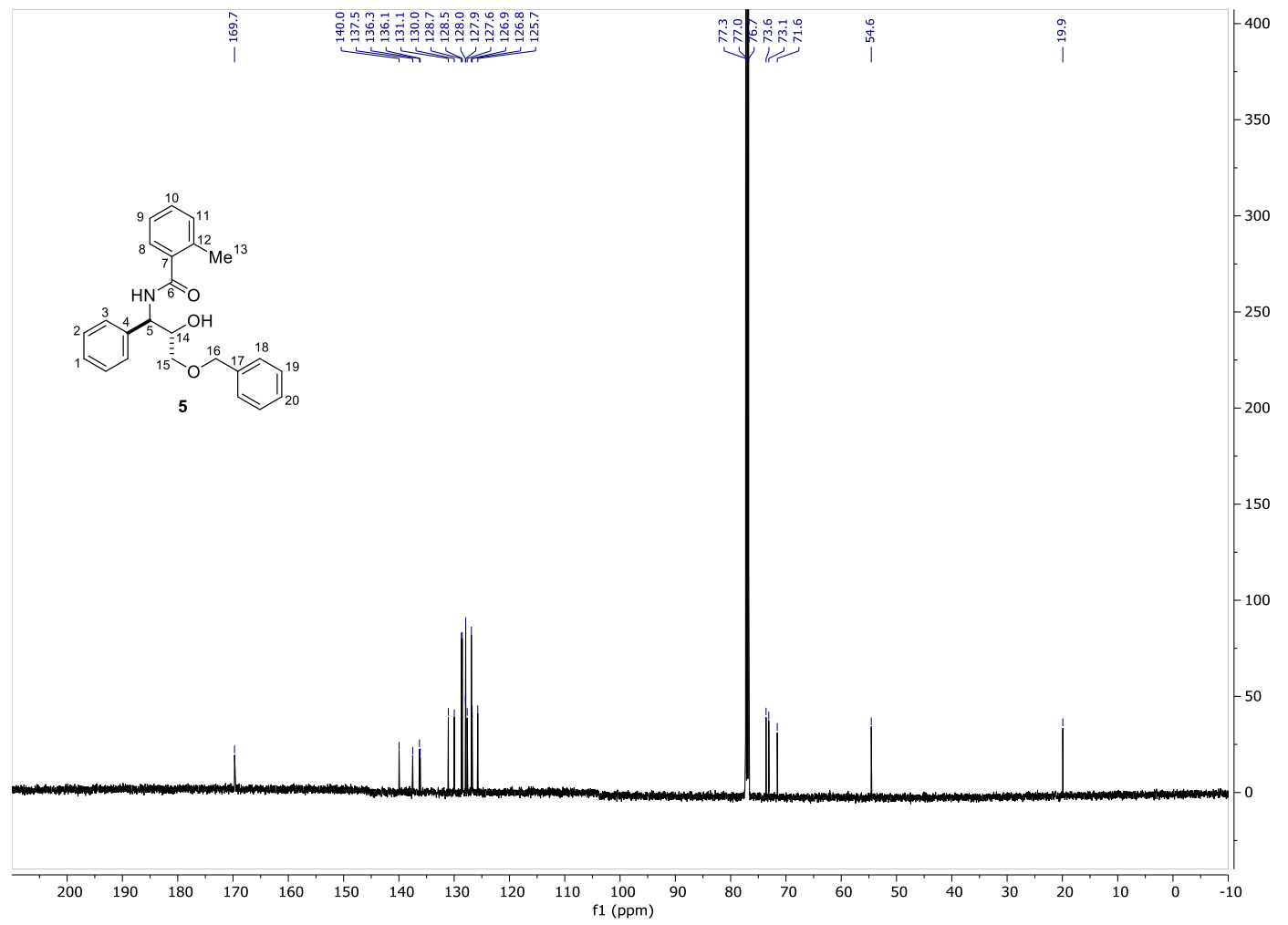




\section{5-racemic}

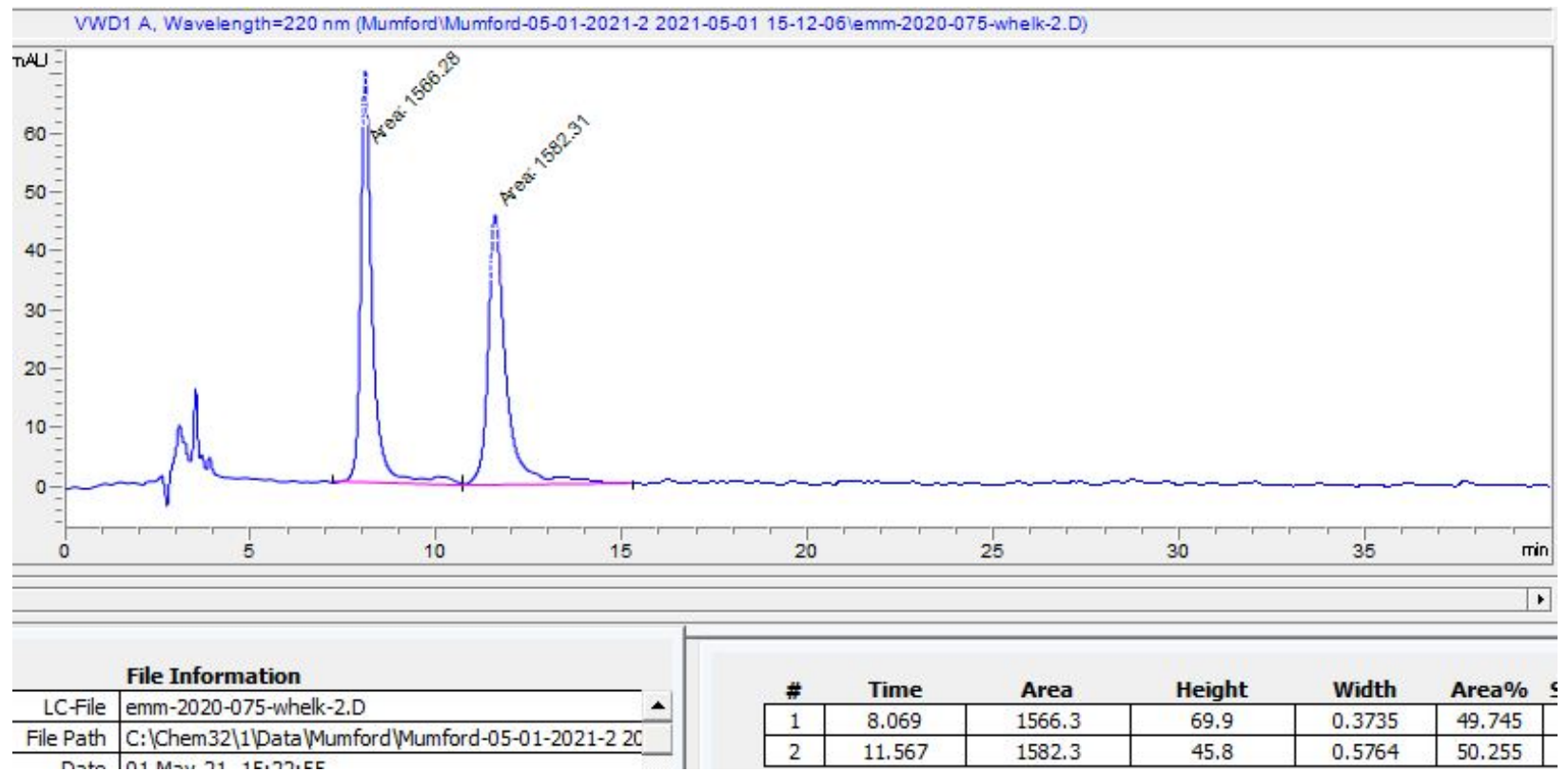

\section{5- $(R, S)$}

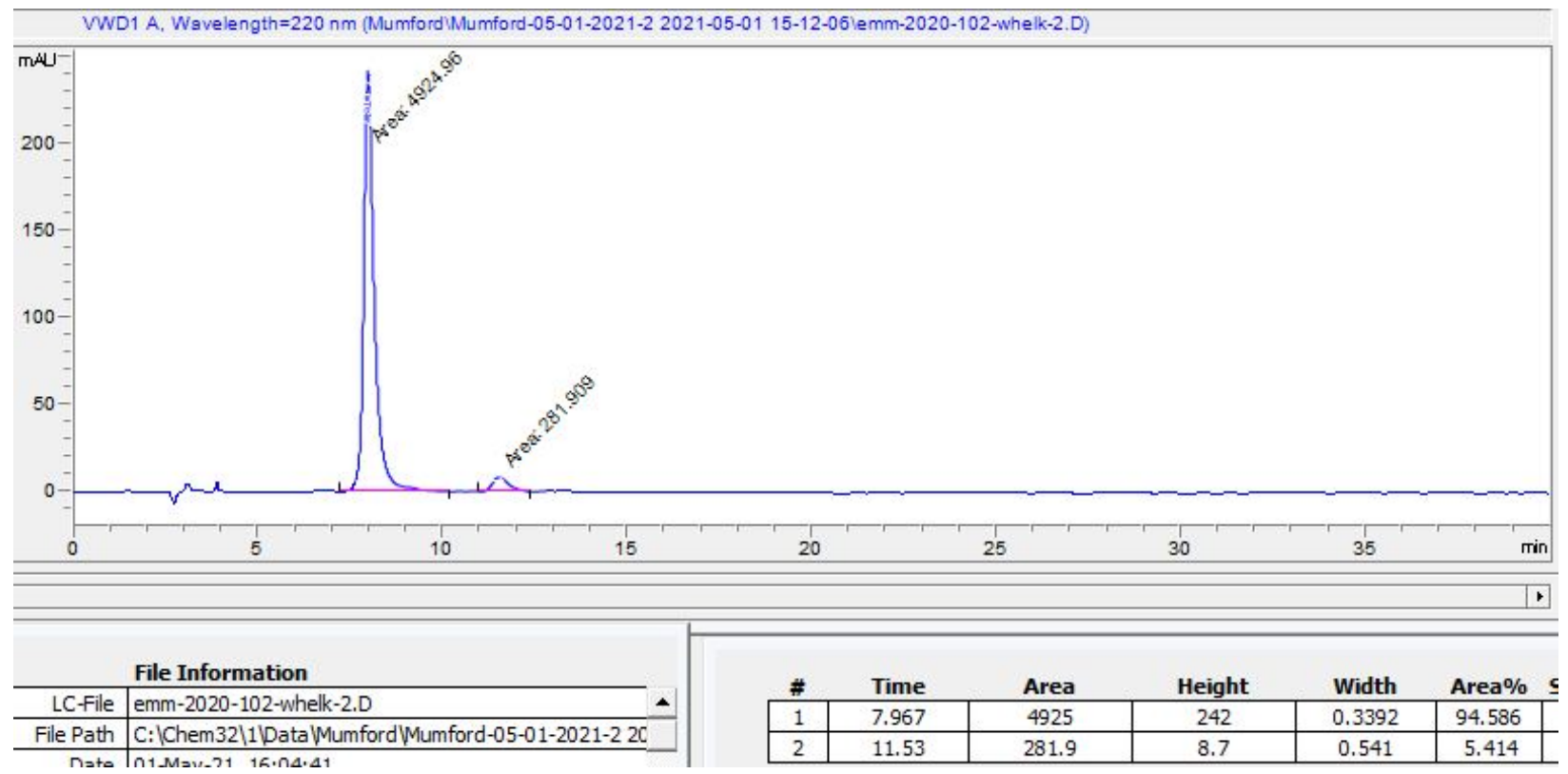


$6{ }^{1} \mathrm{H}$ NMR (500 MHz, MeOH-d 4 )

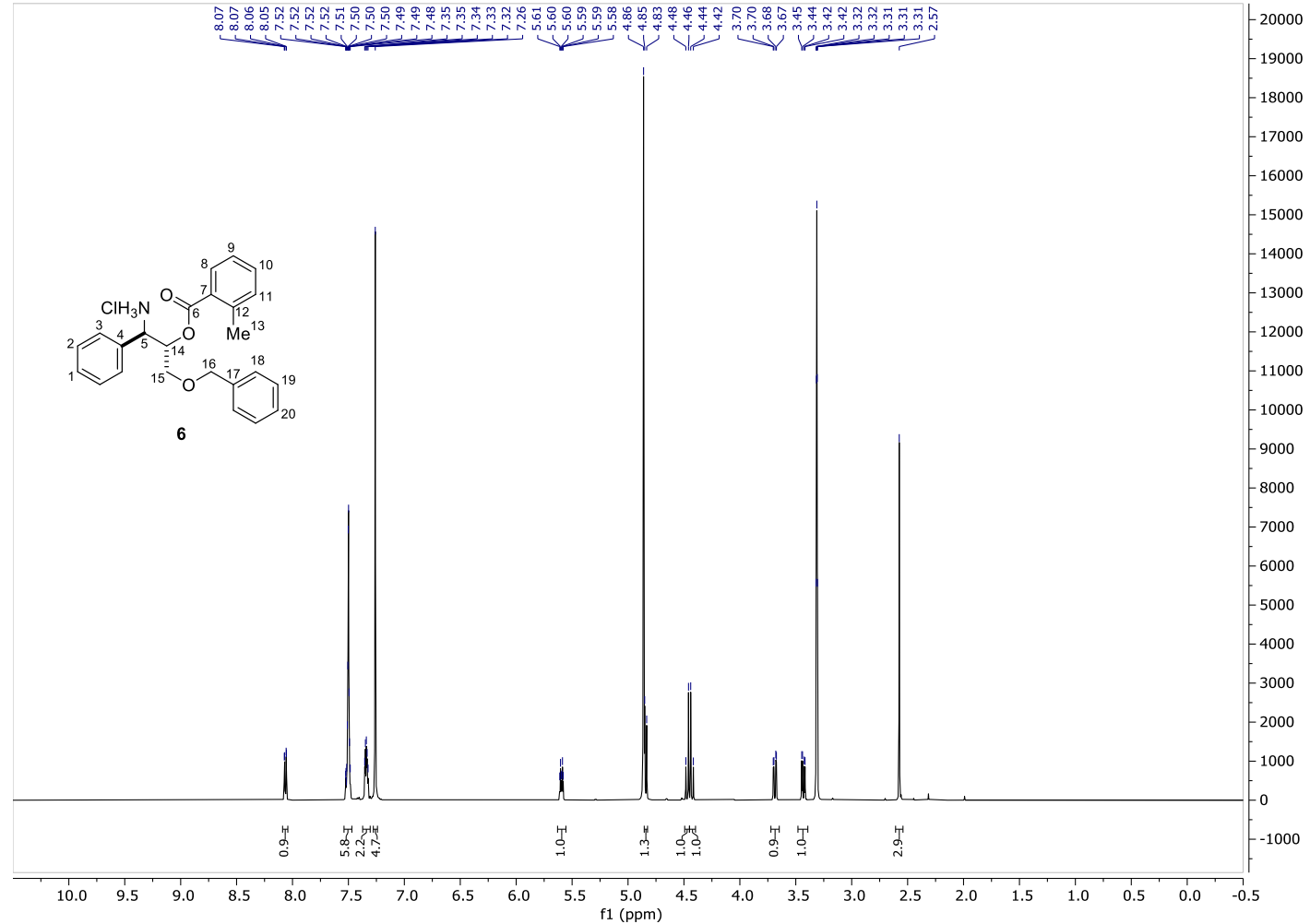

$6{ }^{13} \mathrm{C}$ NMR (126 MHz, MeOH-d 4 )

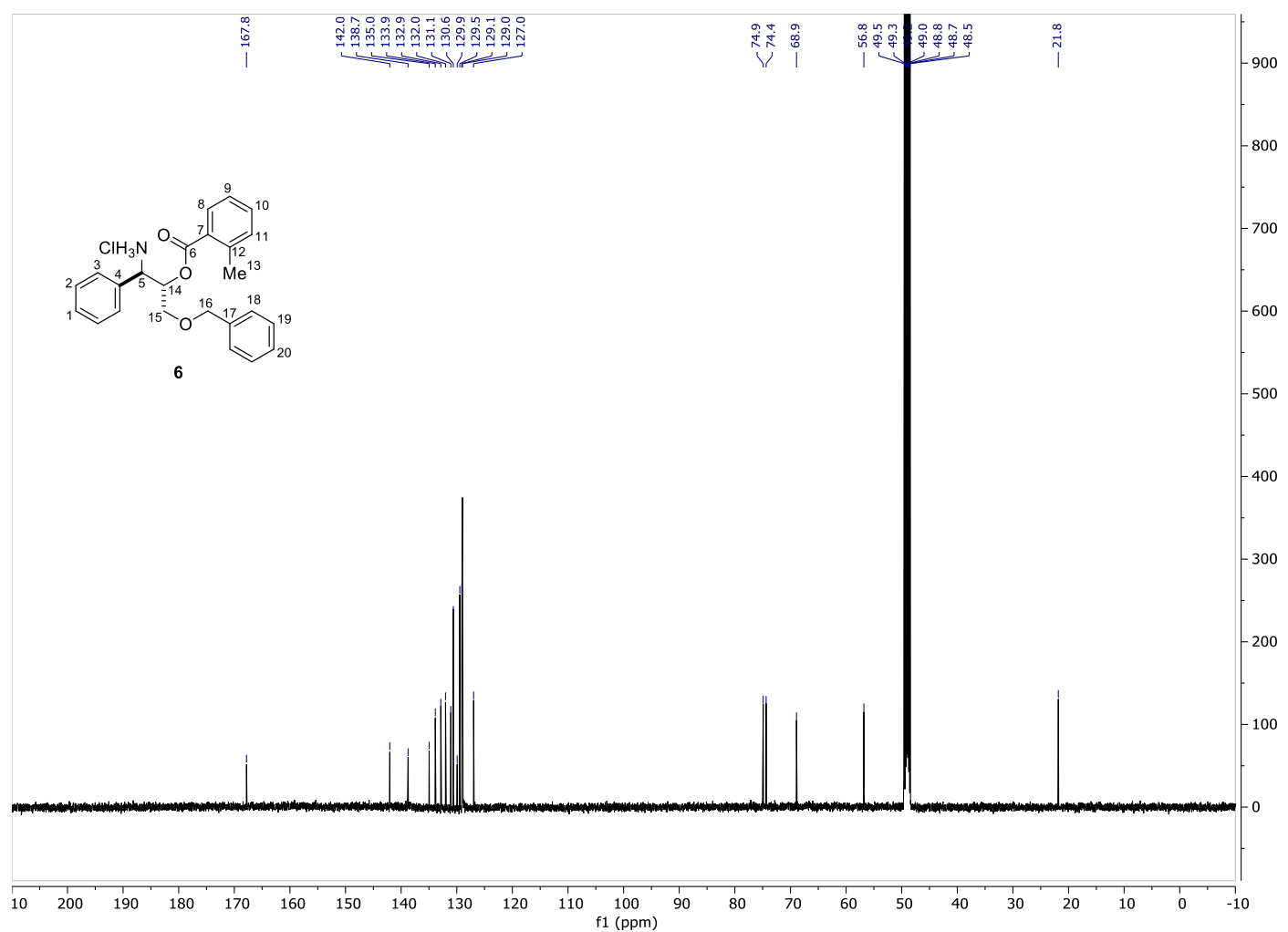


7-i ${ }^{1} \mathrm{H}$ NMR $\left(500 \mathrm{MHz}, \mathrm{CDCl}_{3}\right)$

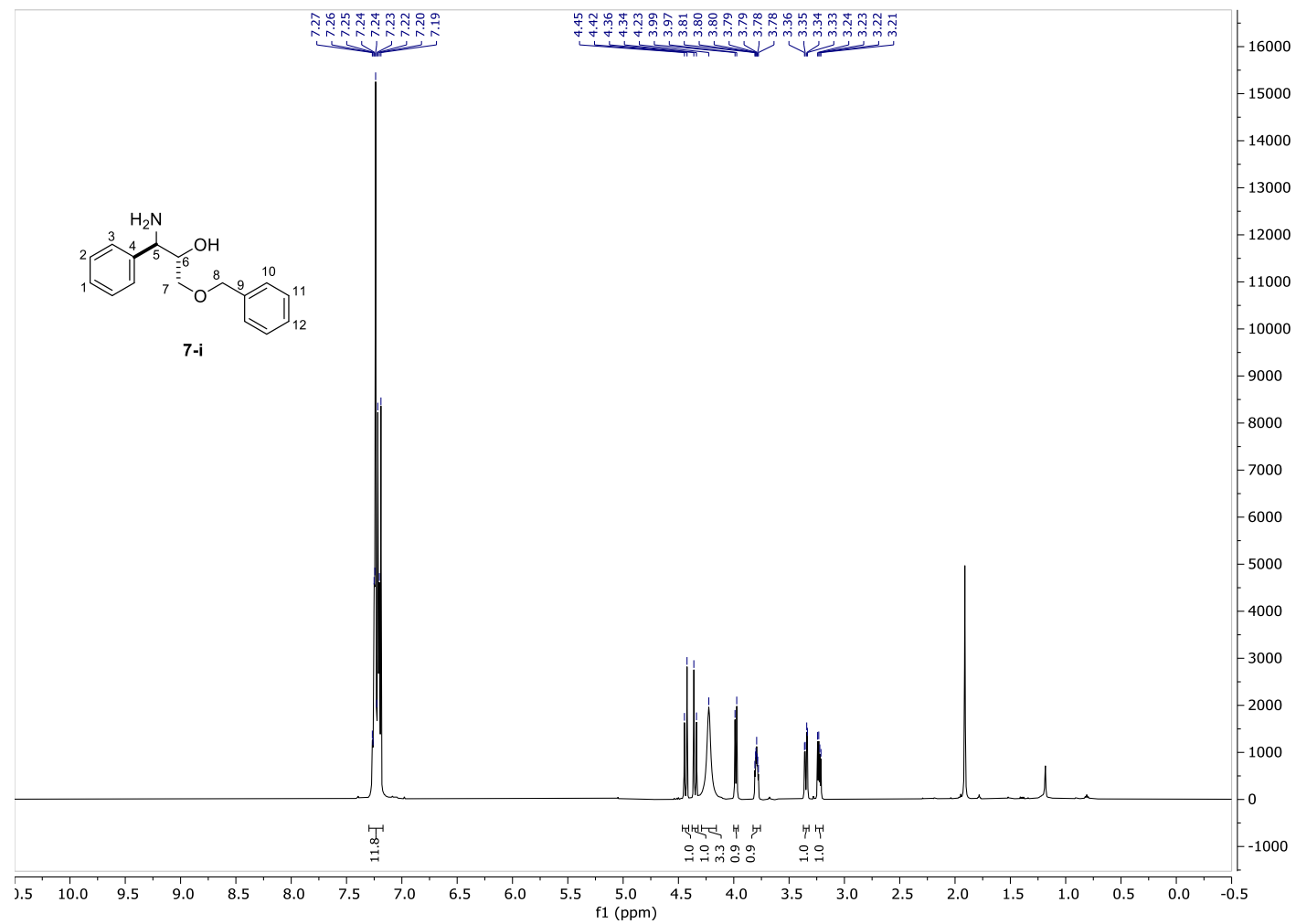

7-i ${ }^{13} \mathrm{C} \mathrm{NMR}\left(126 \mathrm{MHz}, \mathrm{CDCl}_{3}\right)$

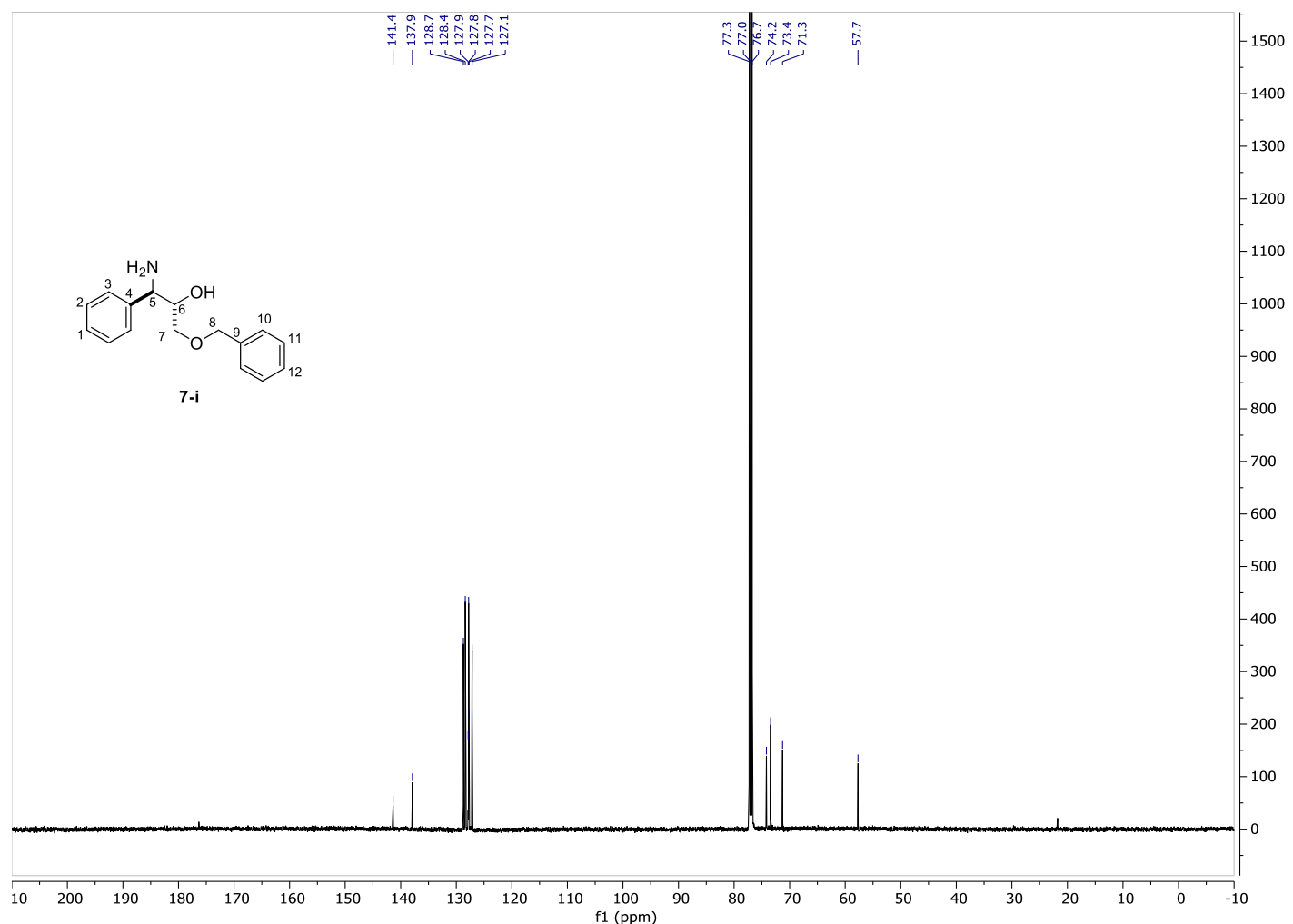


$7{ }^{1} \mathrm{H}$ NMR (500 MHz, MeOH-d4)

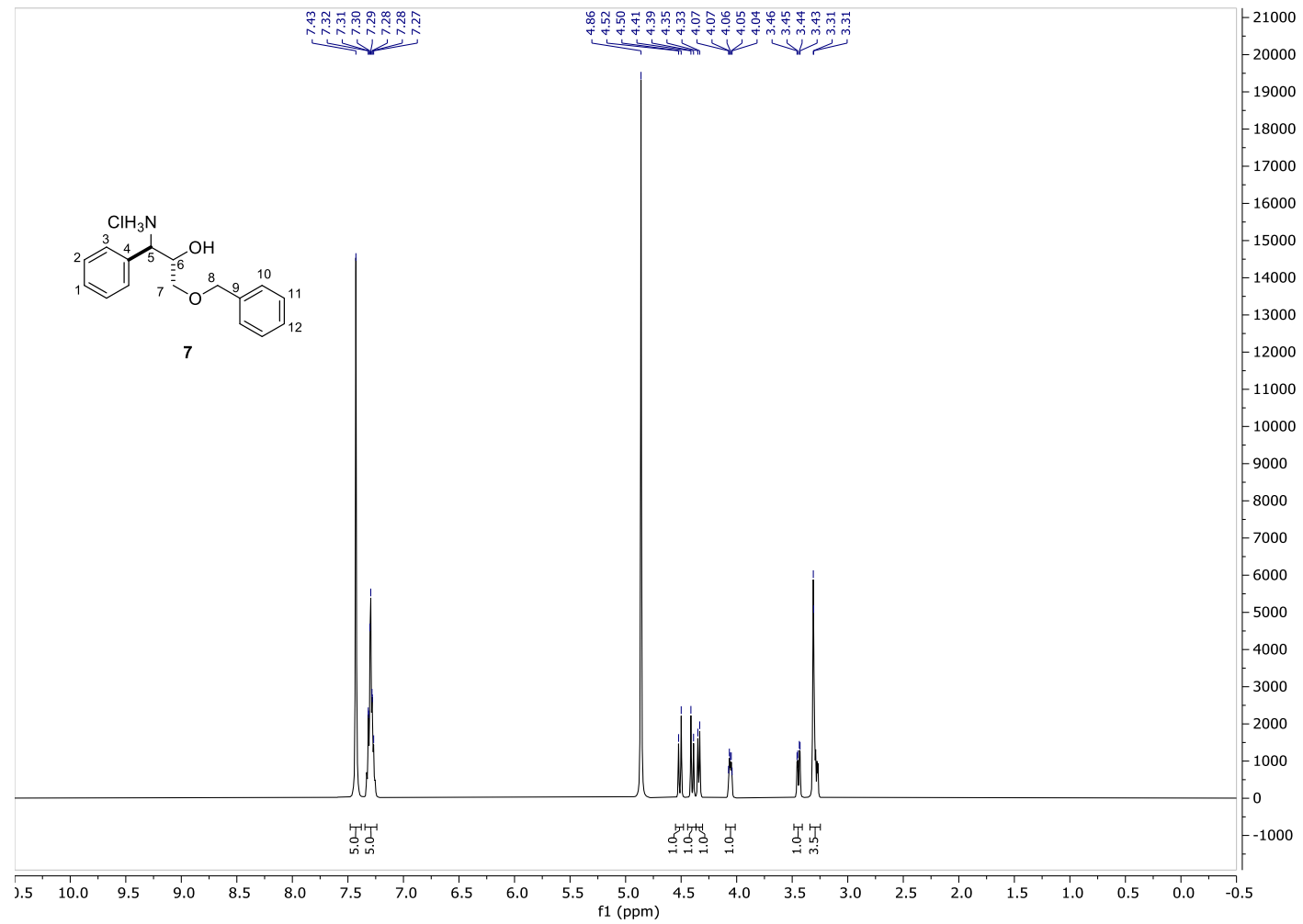

$7{ }^{13} \mathrm{C}$ NMR (126 MHz, MeOH-d 4 )

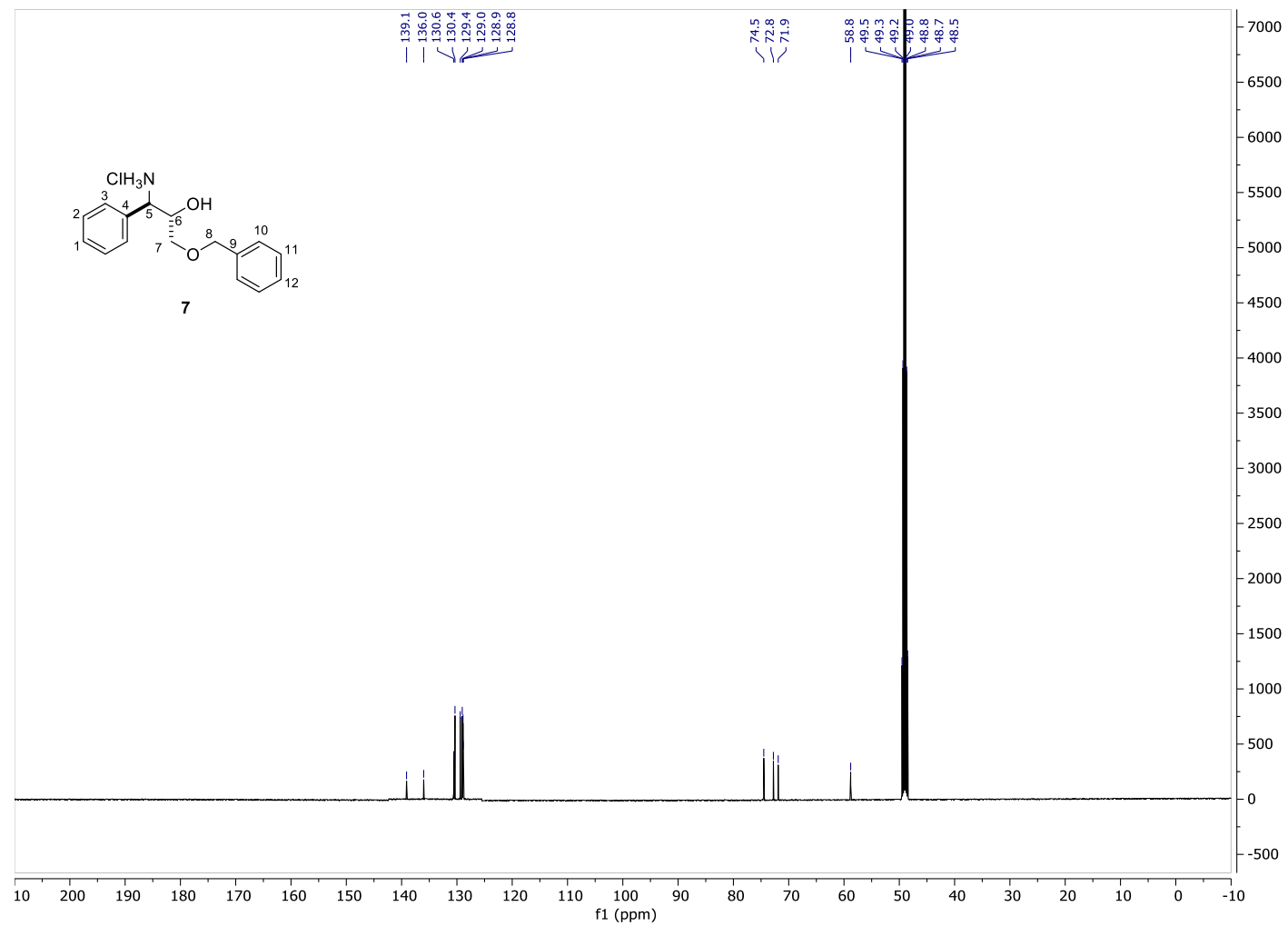


6-racemic (via acylation of 7)

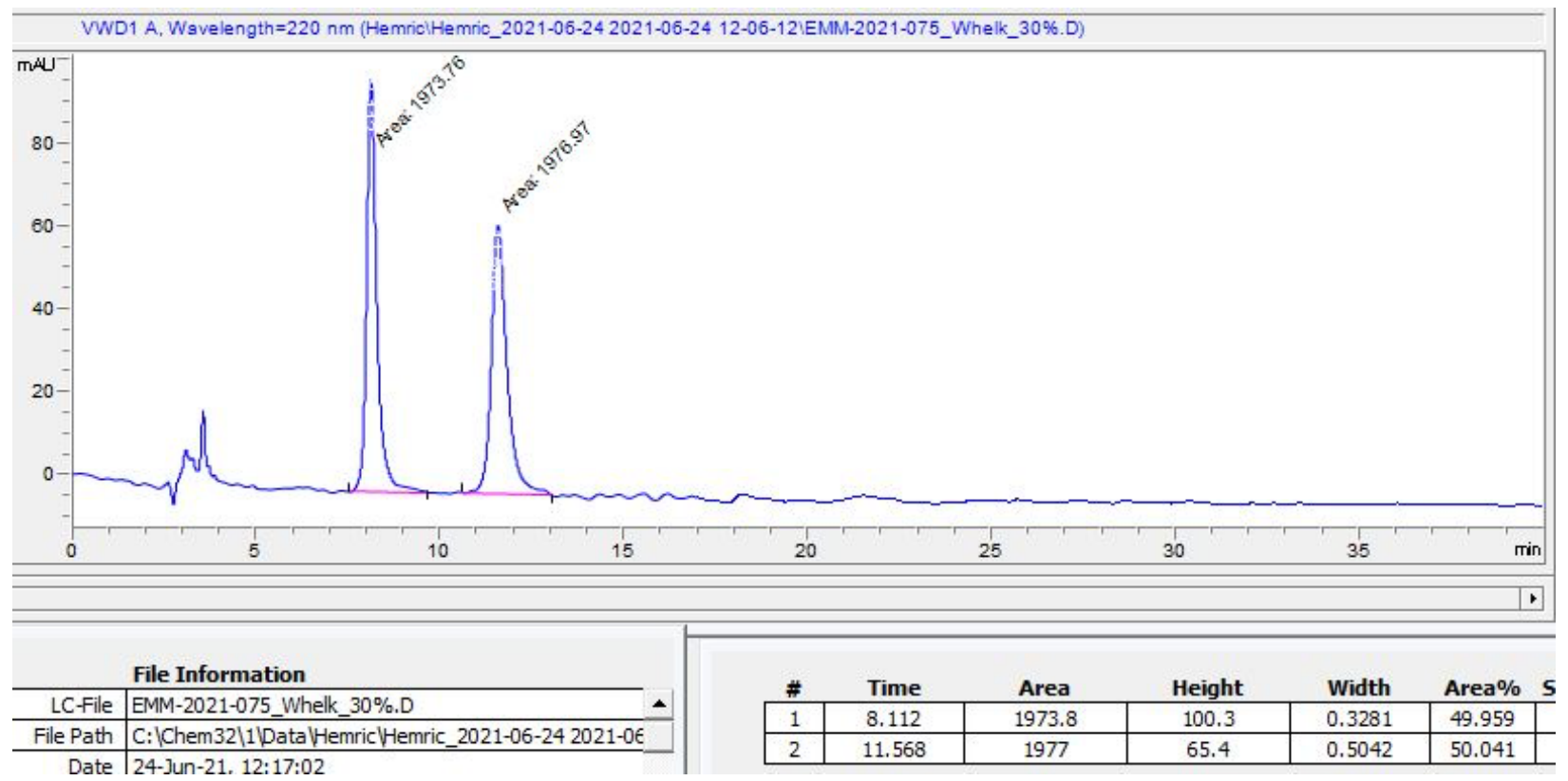

6-( $(R, S)$ (via acylation of 7$)$

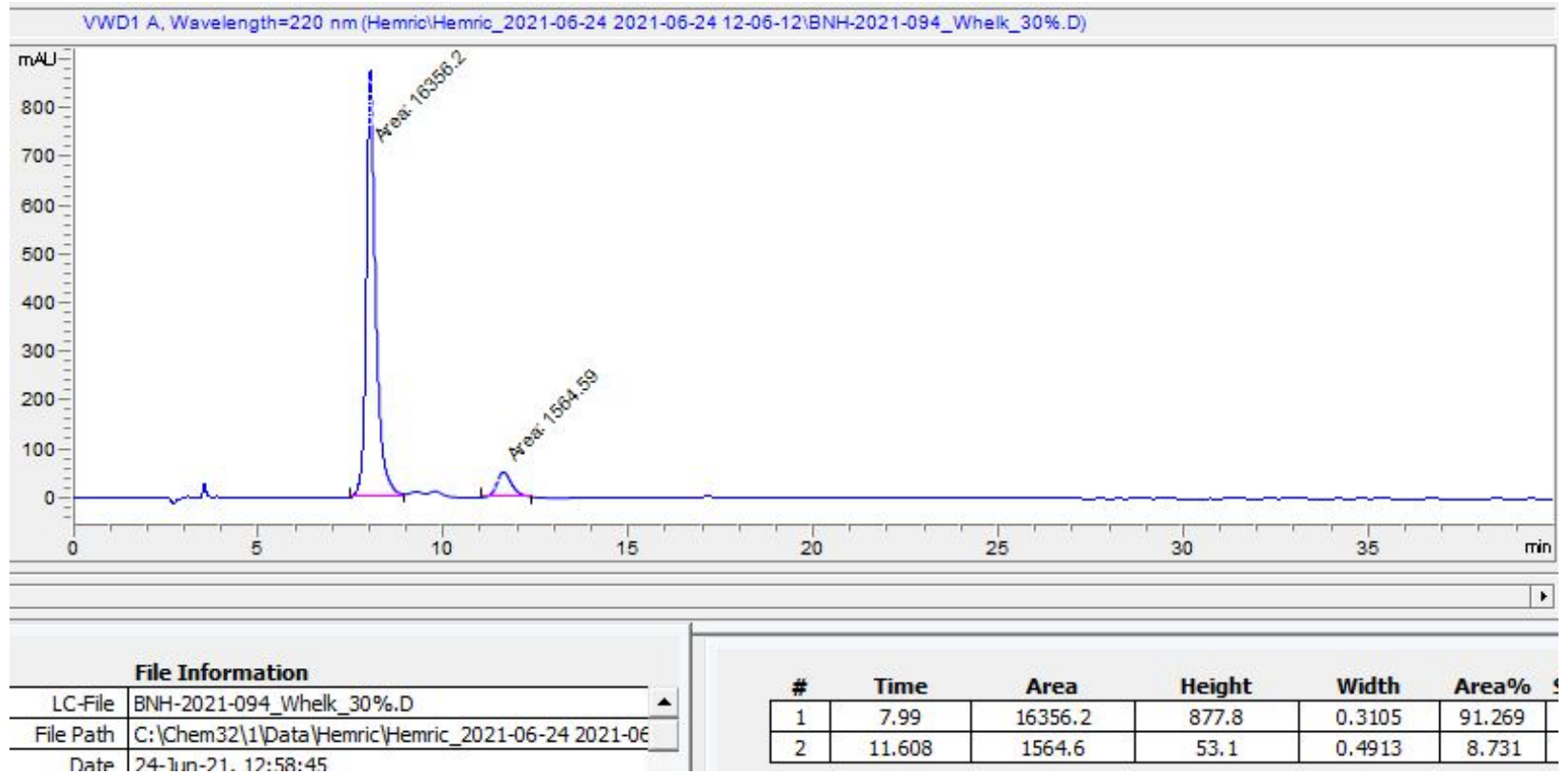


$9{ }^{1} \mathrm{H}$ NMR $\left(500 \mathrm{MHz}, \mathrm{CDCl}_{3}\right)$

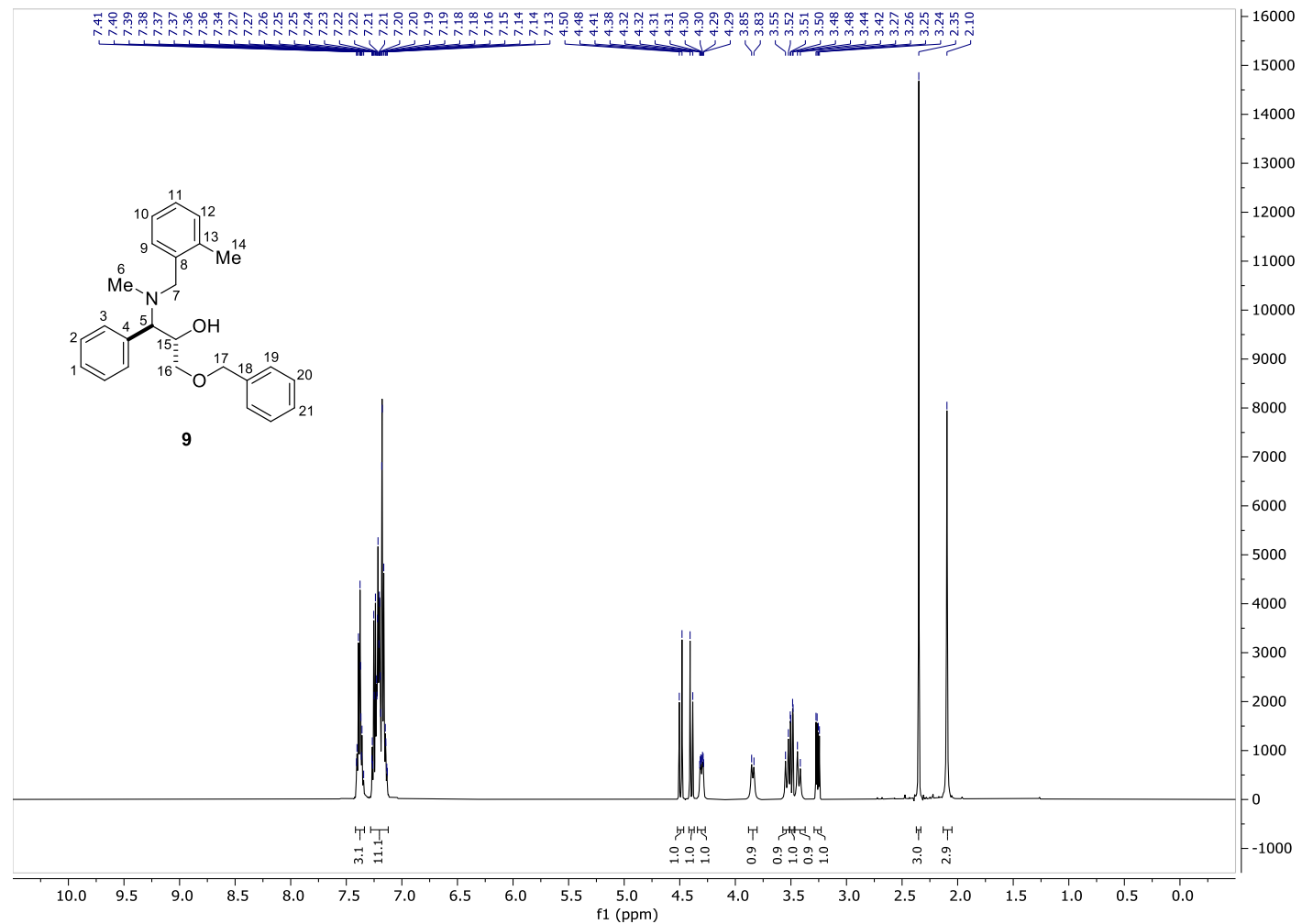

$\left.9{ }^{13} \mathrm{C} \mathrm{NMR} \mathrm{(126} \mathrm{MHz,} \mathrm{CDCl}_{3}\right)$

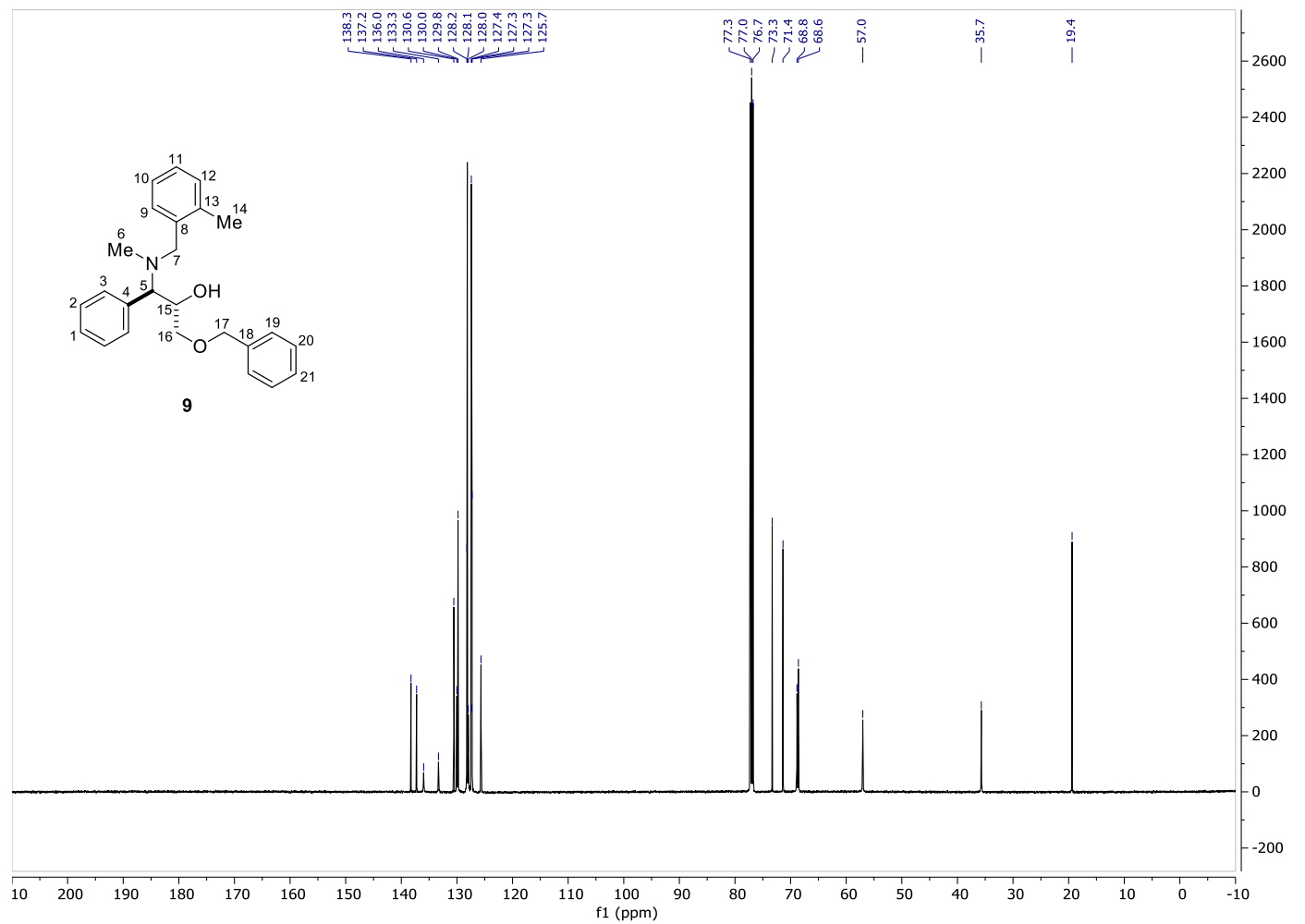




\section{9-racemic}

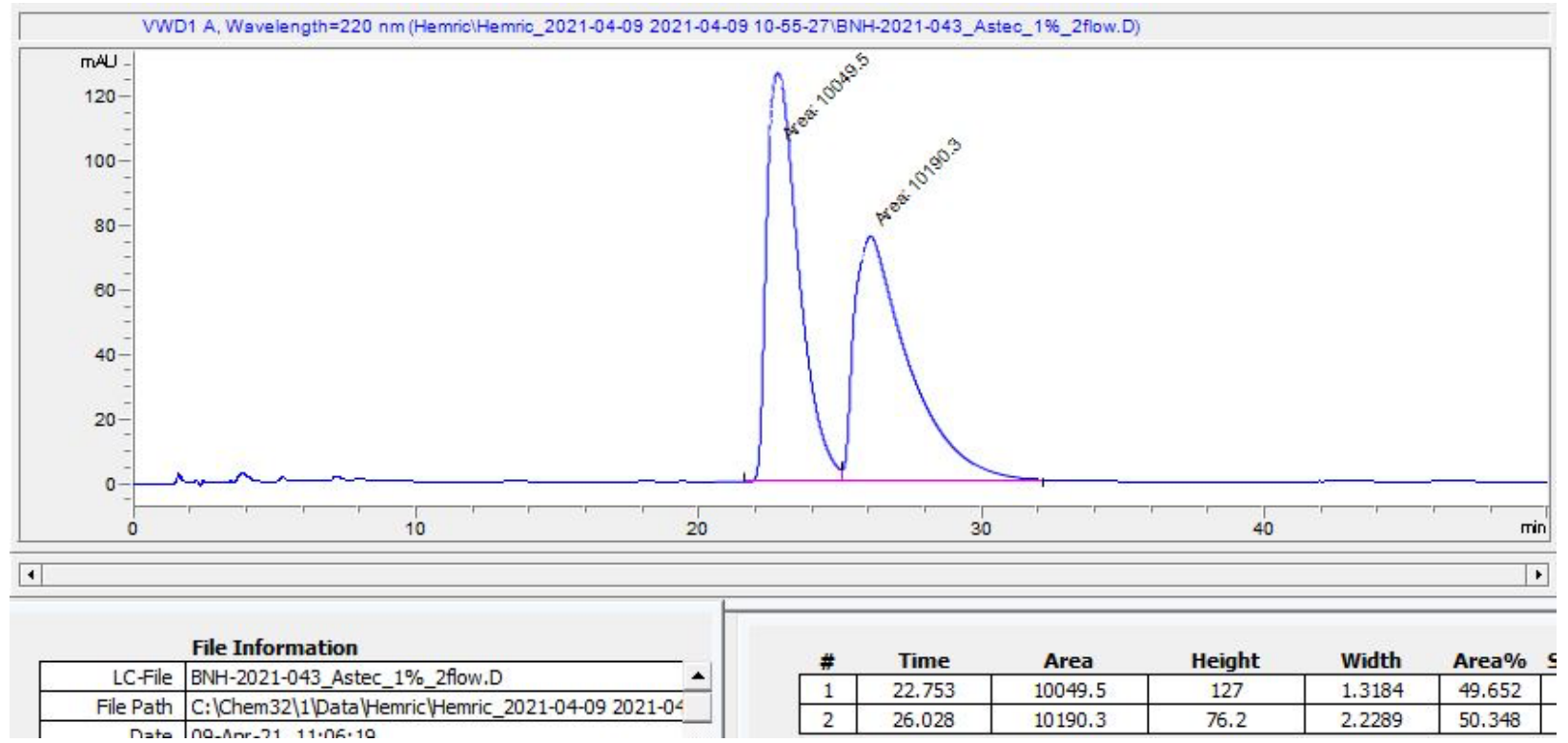

\section{9- $(R, R)$}

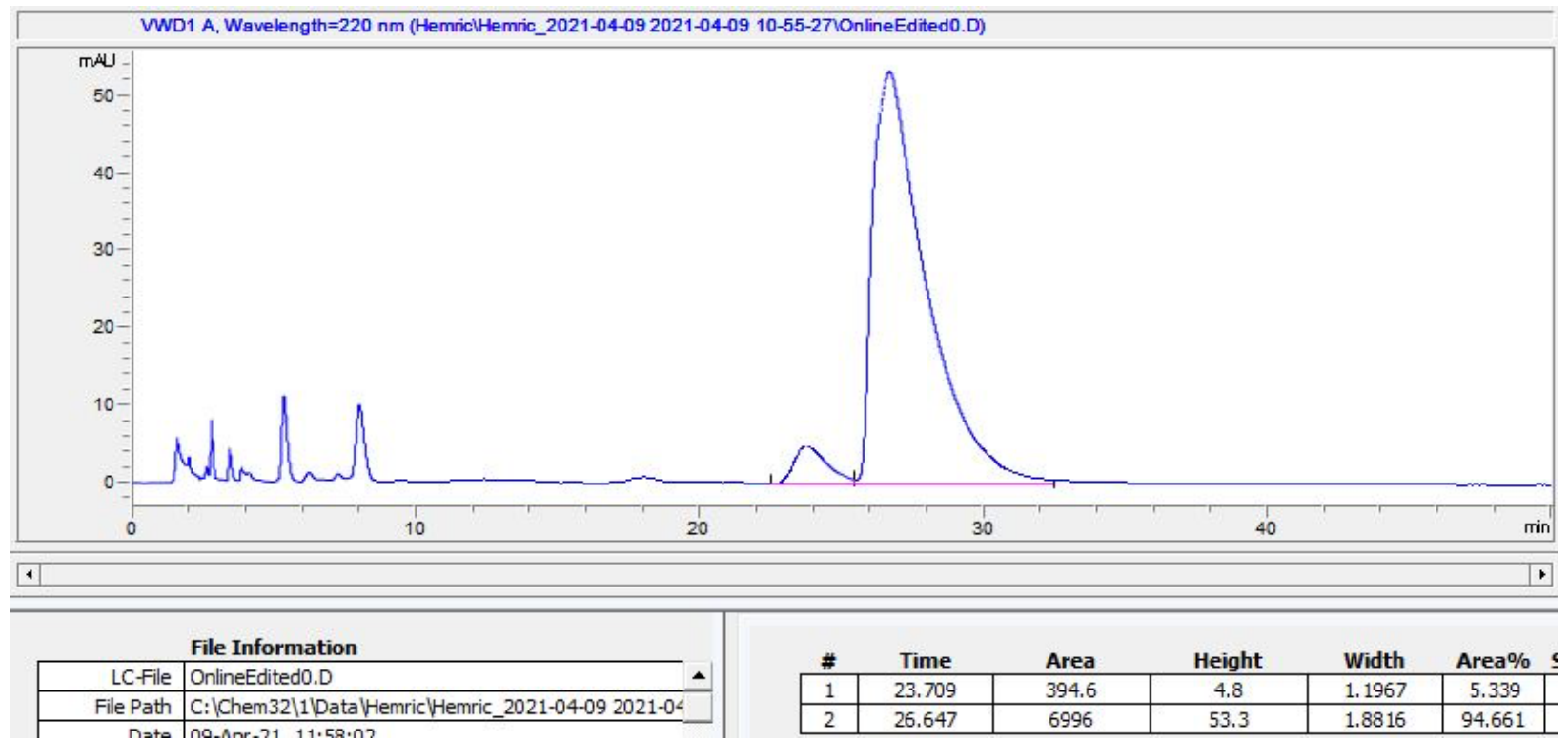


$10{ }^{1} \mathrm{H}$ NMR $\left(500 \mathrm{MHz}, \mathrm{CDCl}_{3}, 50{ }^{\circ} \mathrm{C}\right)$

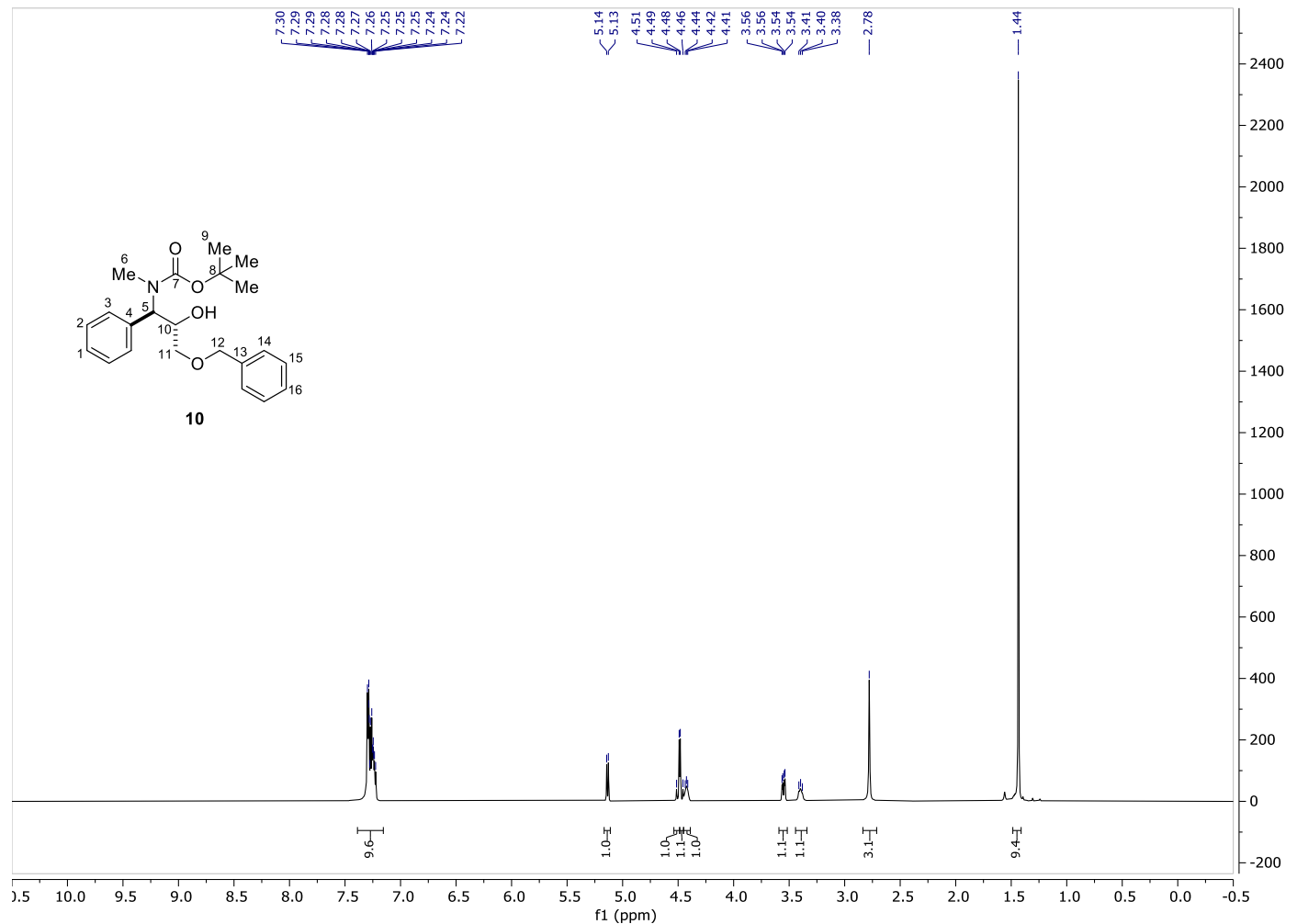

$10{ }^{13} \mathrm{C}$ NMR (126 MHz, $\left.\mathrm{CDCl}_{3}\right)$

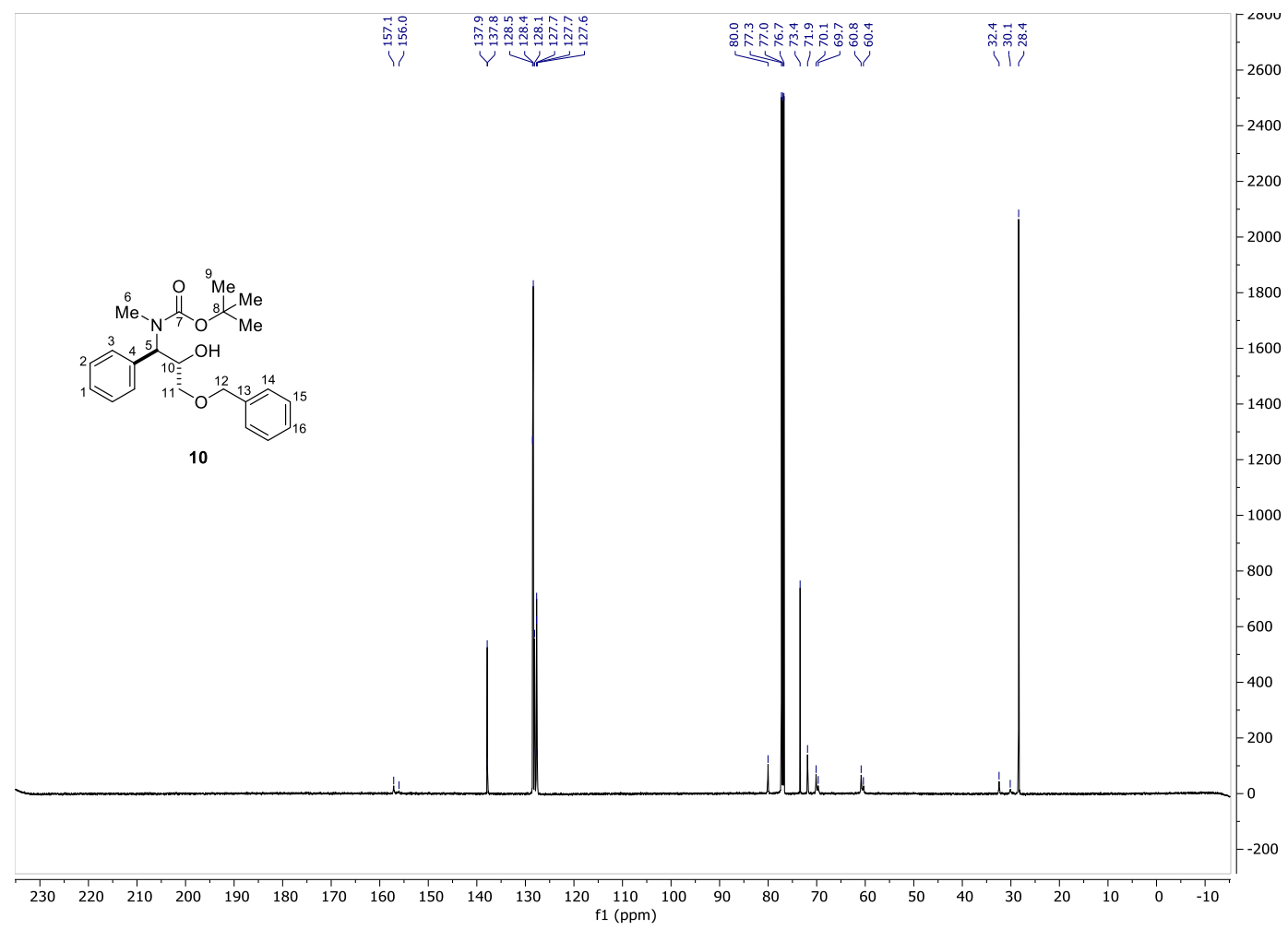


$10{ }^{13} \mathrm{C}$ NMR $\left(126 \mathrm{MHz}, \mathrm{CDCl}_{3}, 50{ }^{\circ} \mathrm{C}\right)$

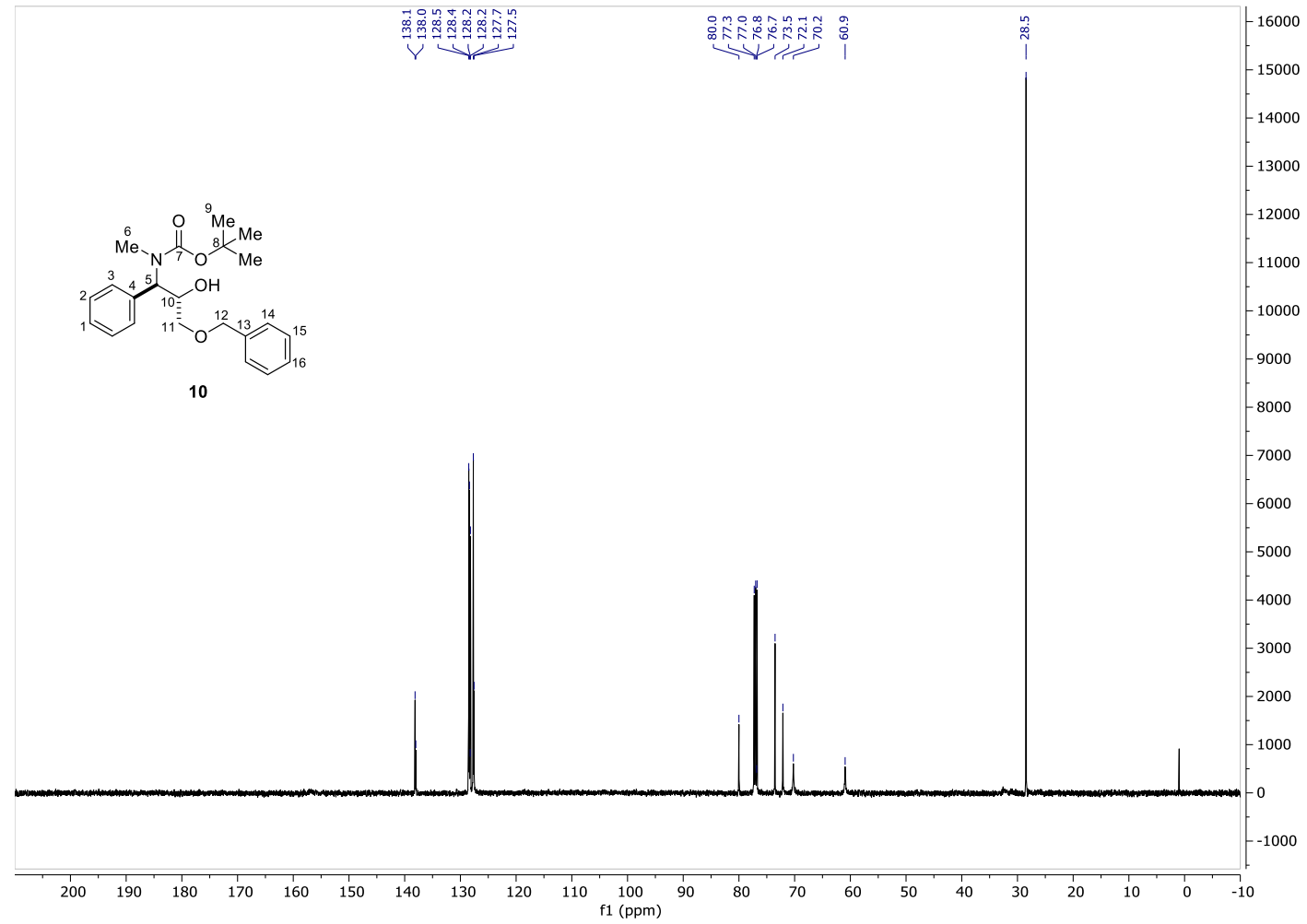




\section{0-racemic}

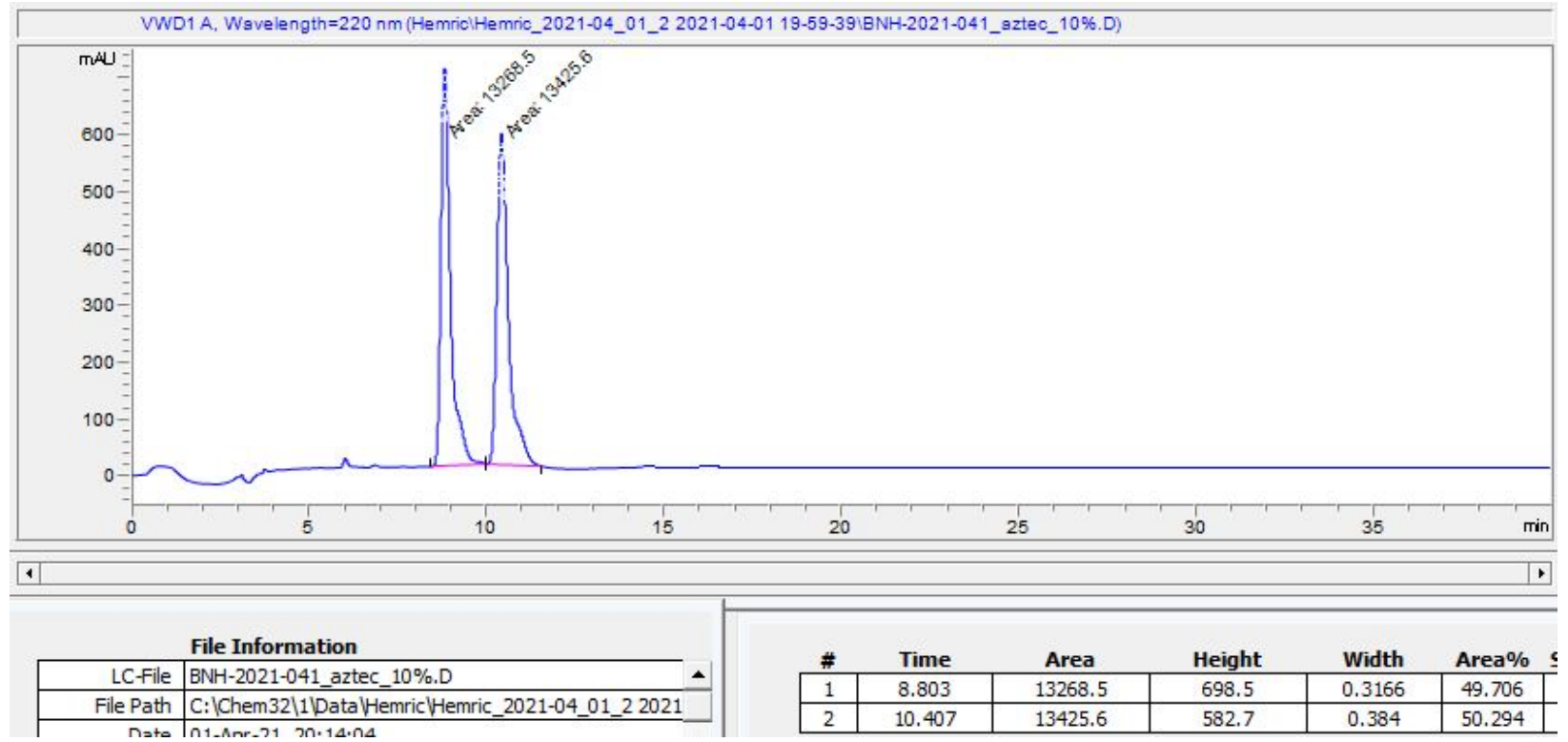

\section{0- $(R, S)$}

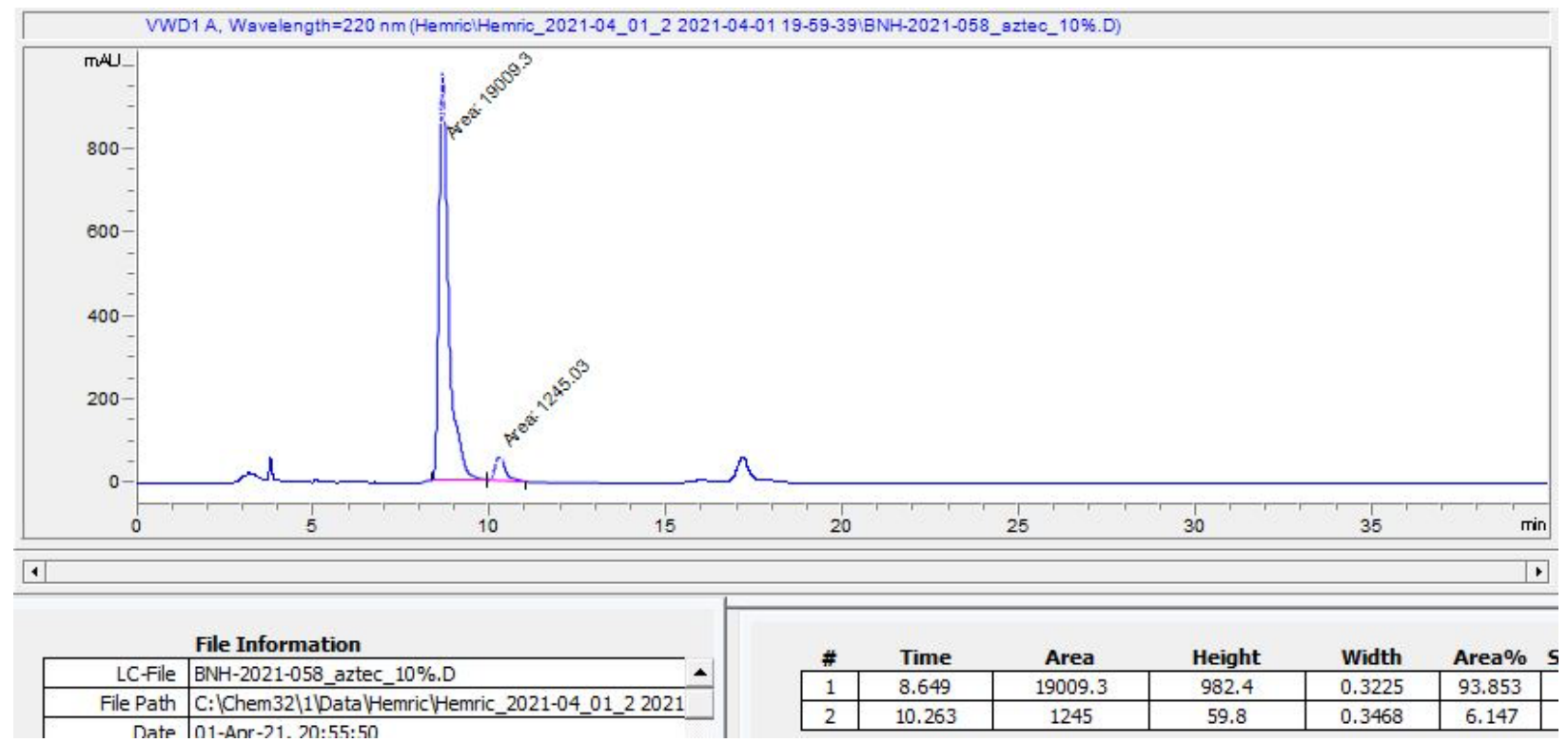


4u ${ }^{1} \mathrm{H}$ NMR (500 MHz, $\mathrm{CDCl}_{3}$ )

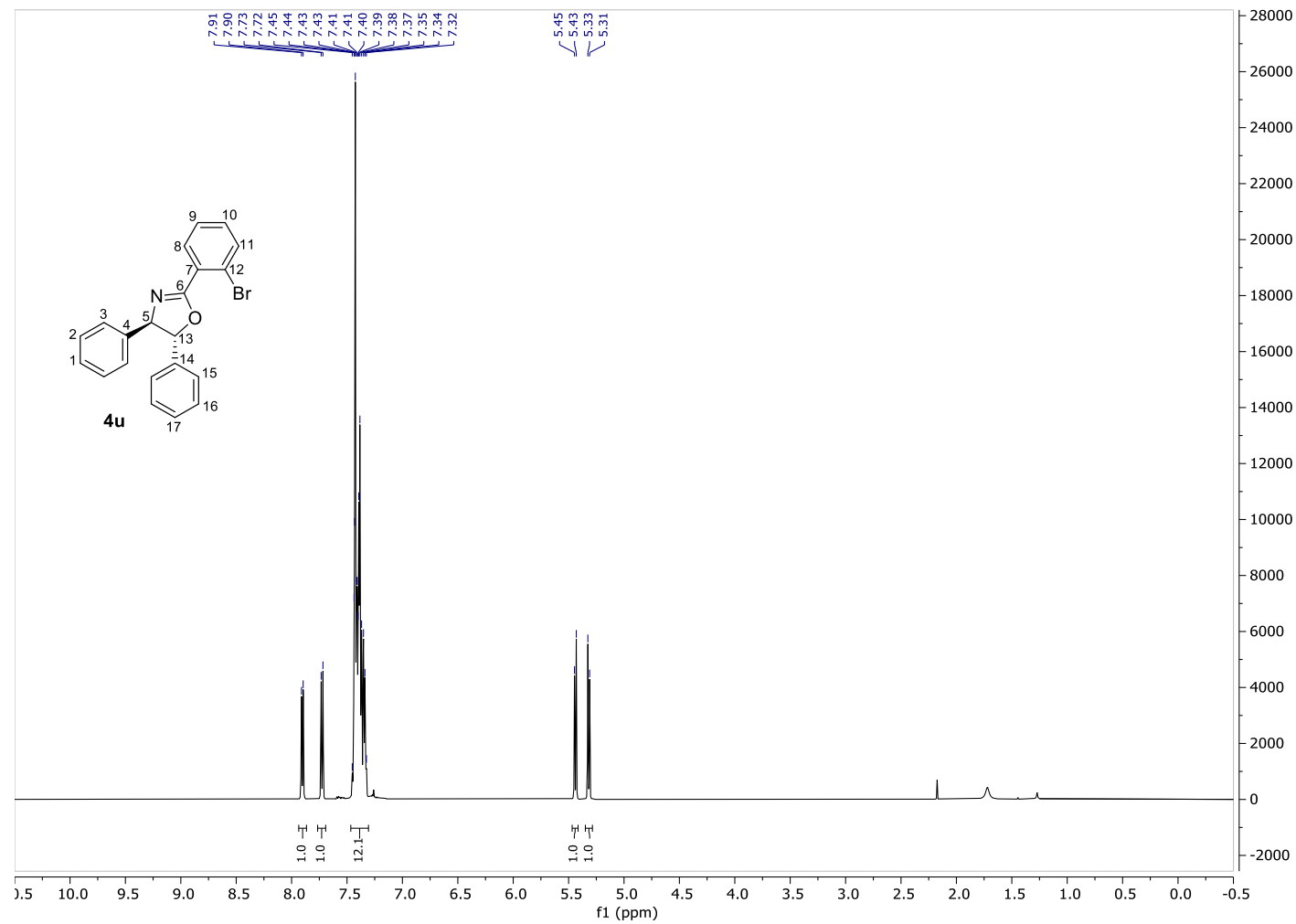

4u ${ }^{13} \mathrm{C}$ NMR (126 MHz, $\mathrm{CDCl}_{3}$ )

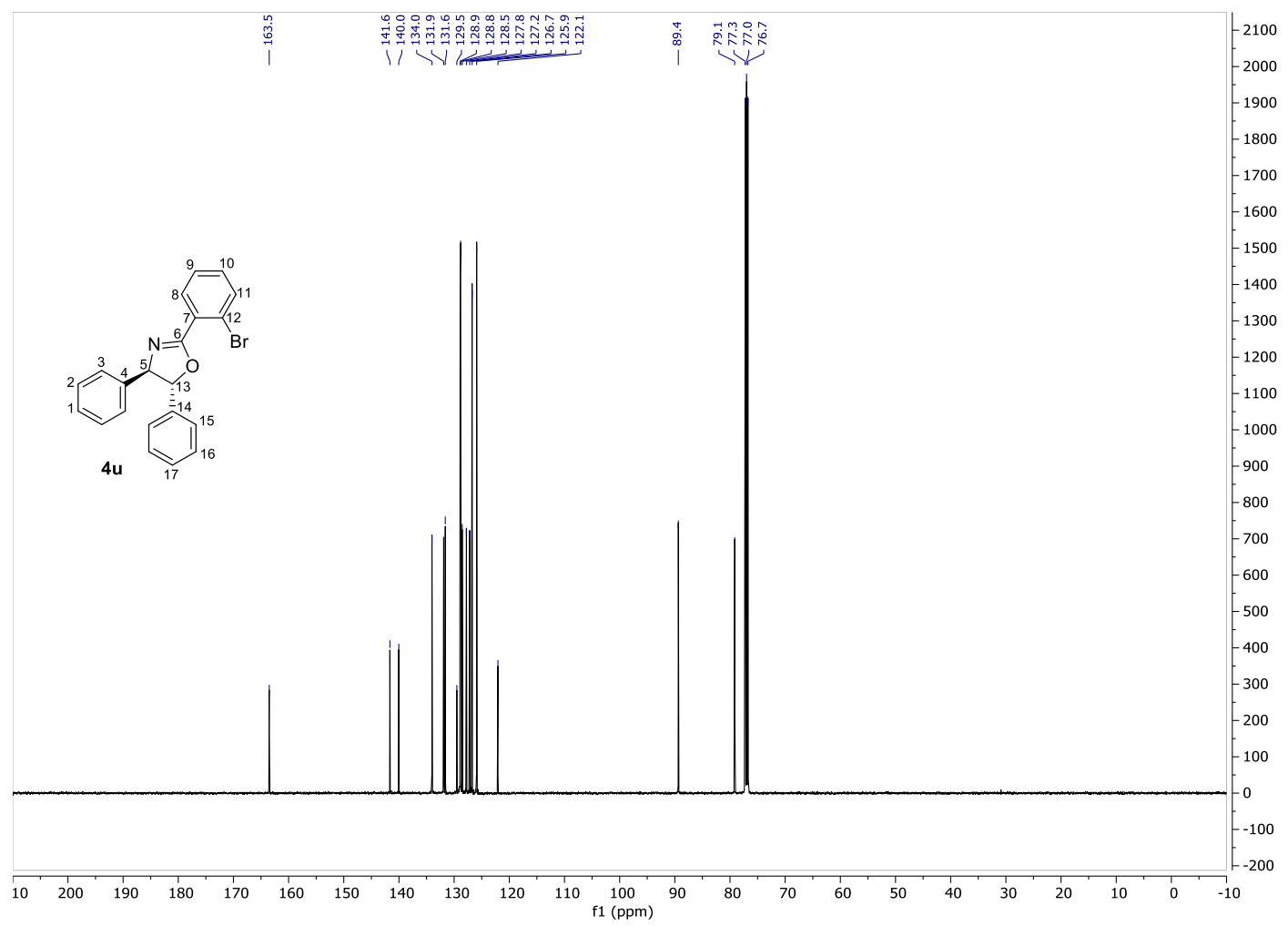




\section{4u-racemic}

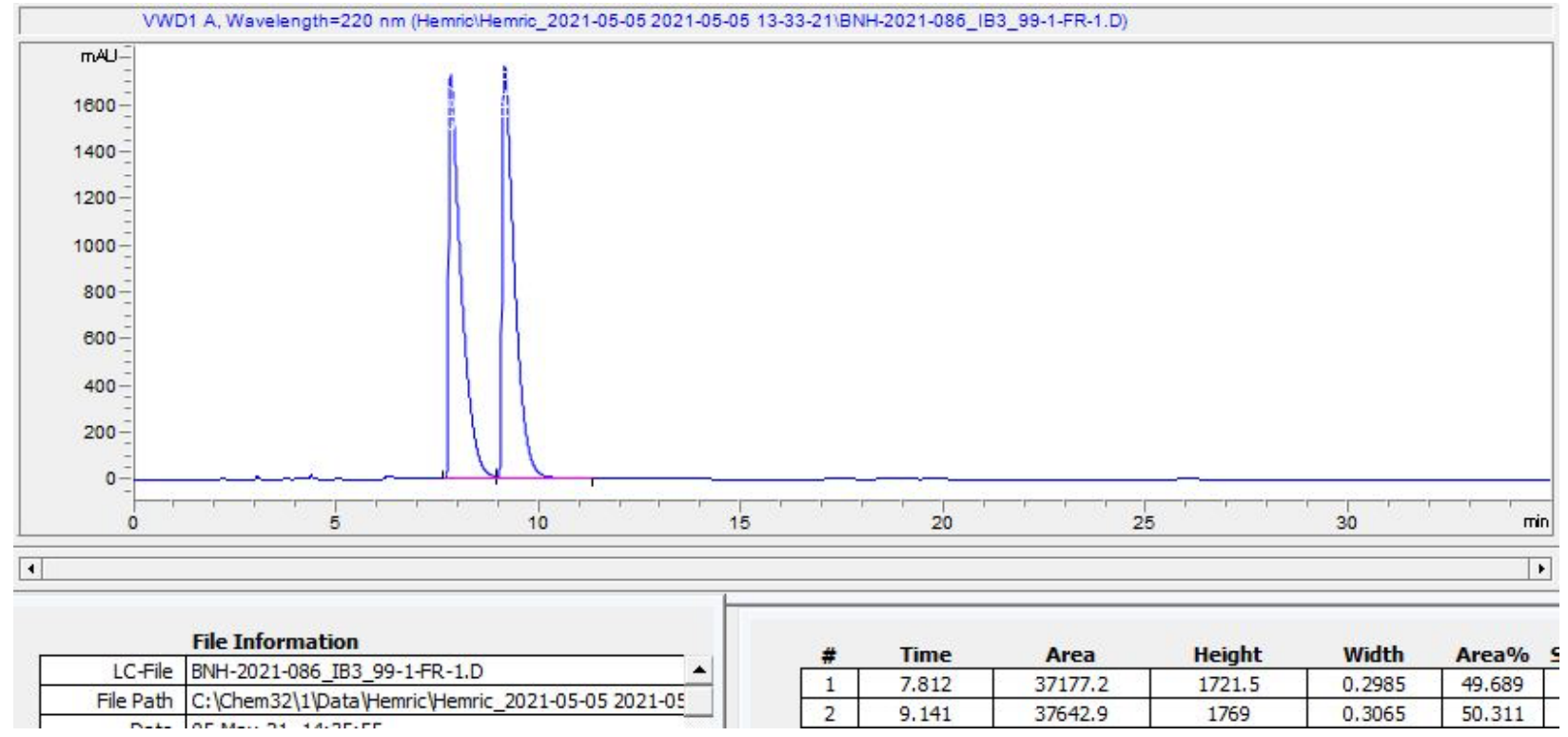

\section{$\mathbf{4 u}-(R, R)$}

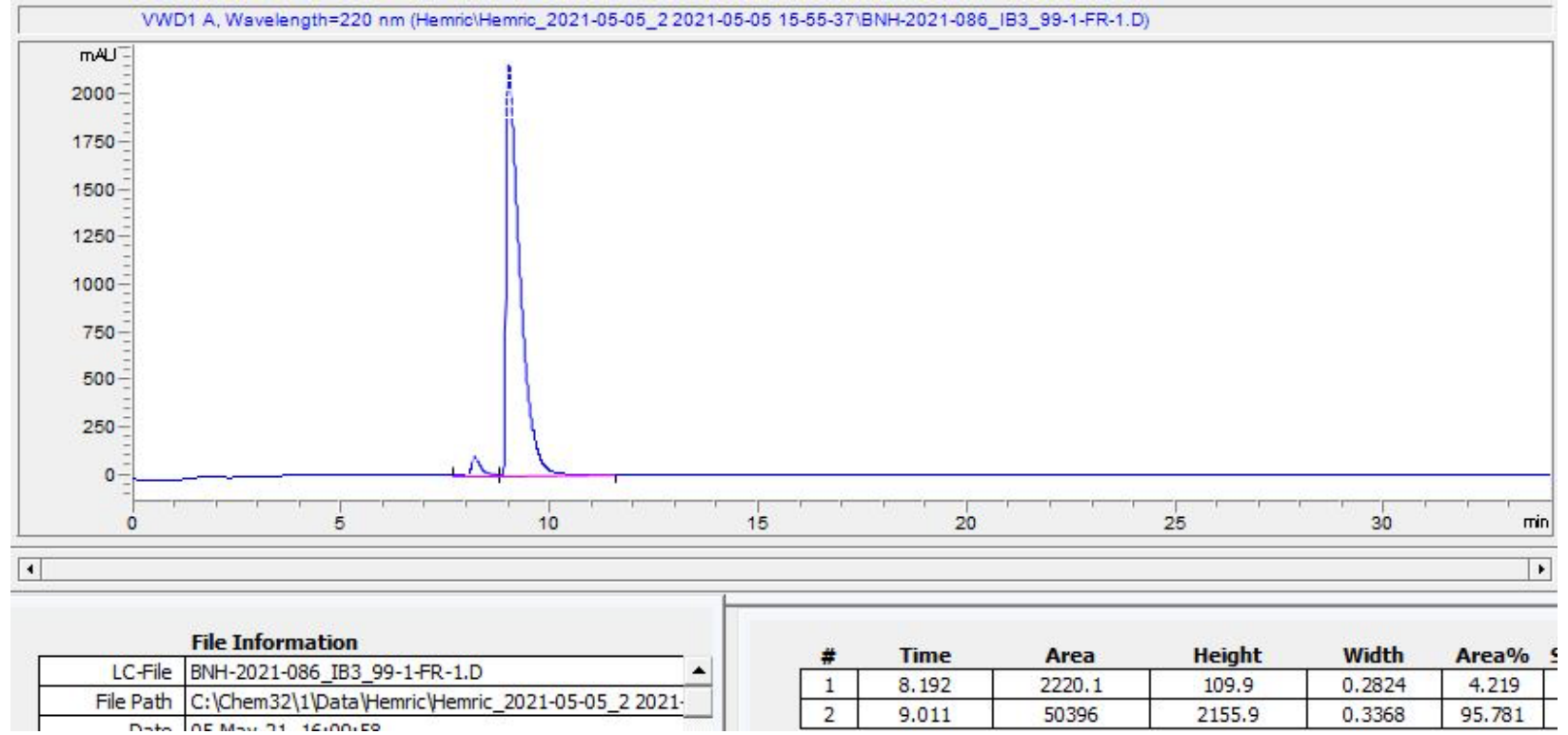




\section{4v ${ }^{1} \mathrm{H}$ NMR $\left(500 \mathrm{MHz}, \mathrm{CDCl}_{3}\right)$}

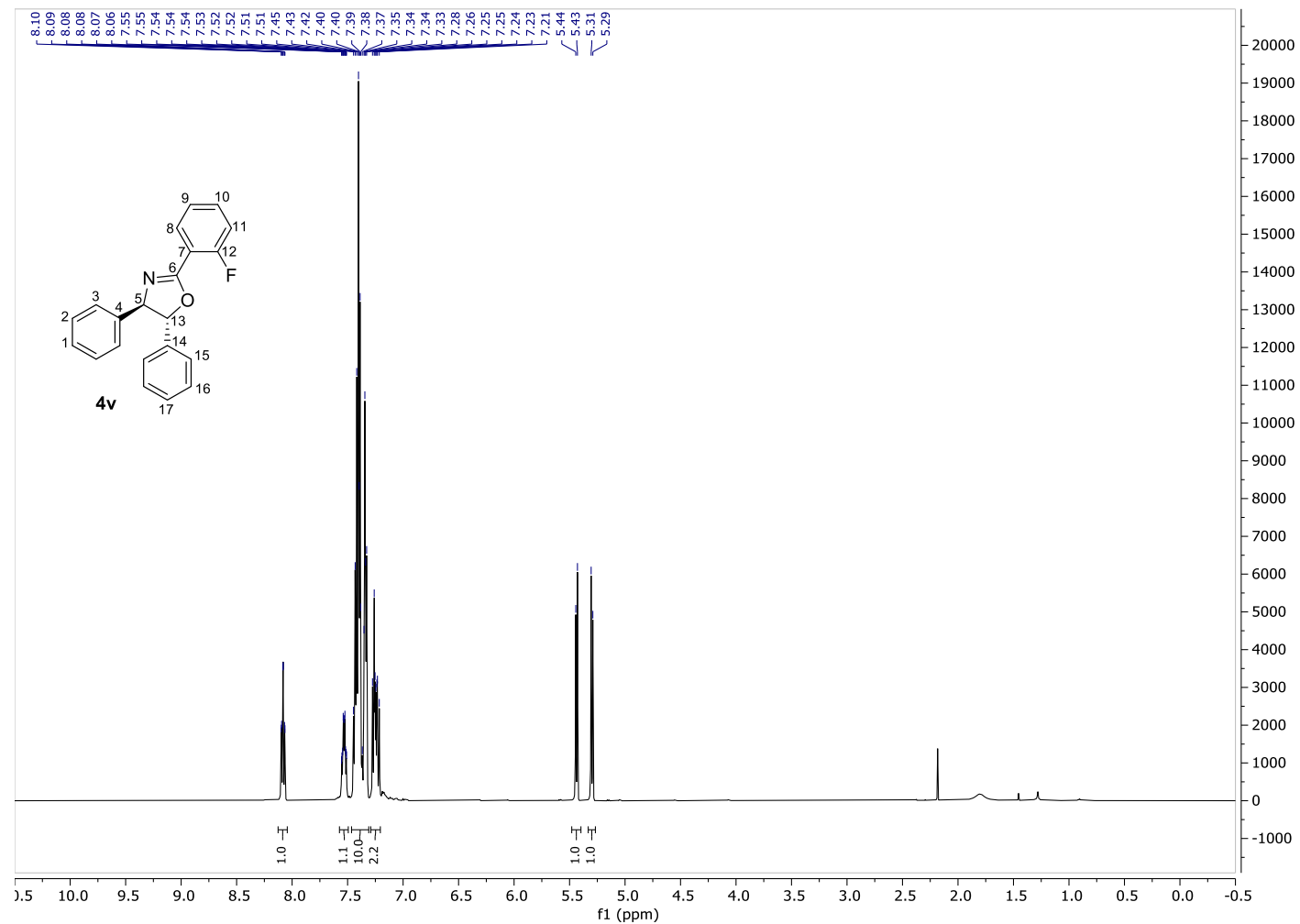

4v ${ }^{13} \mathrm{C}$ NMR (126 MHz, $\left.\mathrm{CDCl}_{3}\right)$

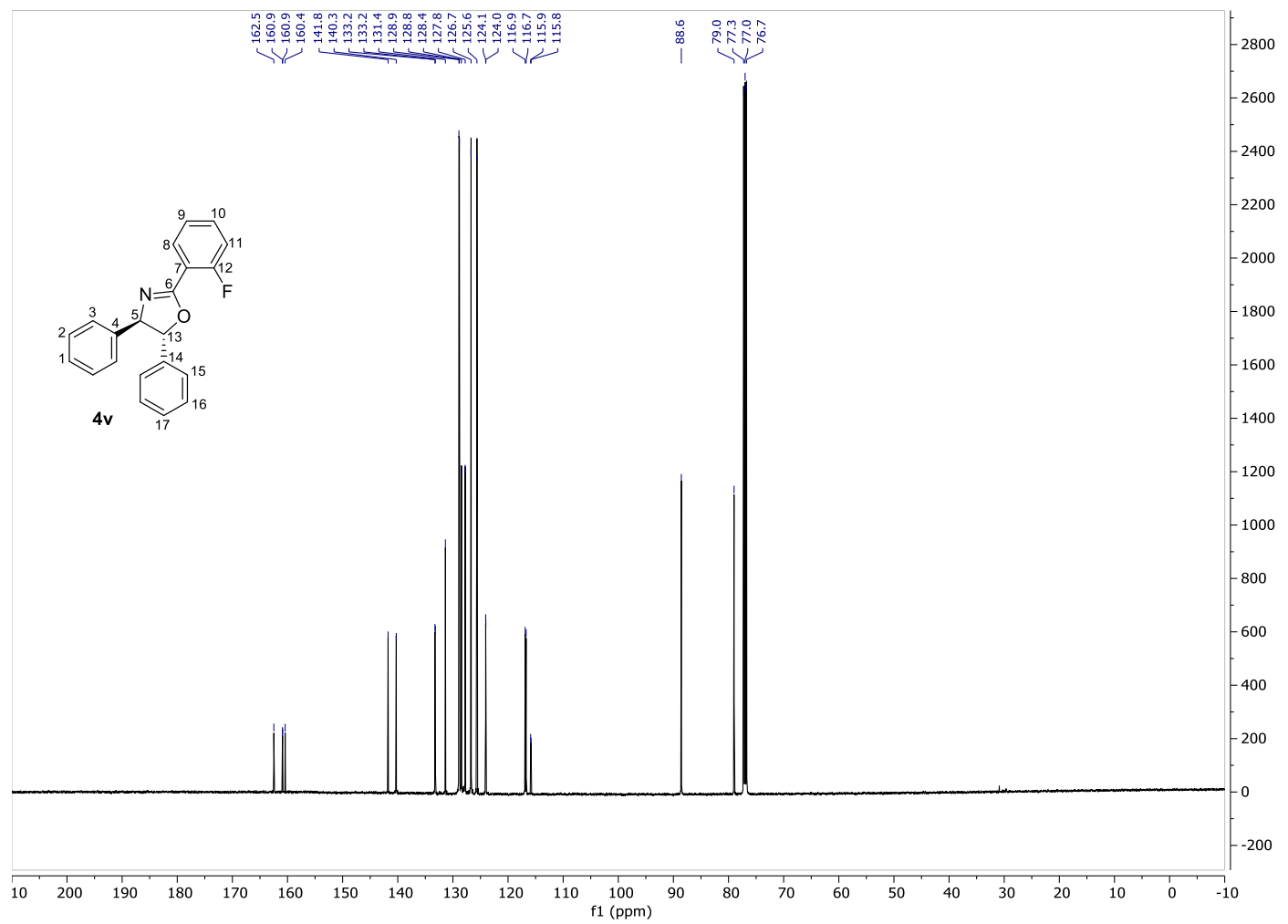


4v ${ }^{19} \mathrm{~F}$ NMR (471 MHz, $\mathrm{CDCl}_{3}$ with $\mathrm{C}_{6} \mathrm{~F}_{5}$ as standard)

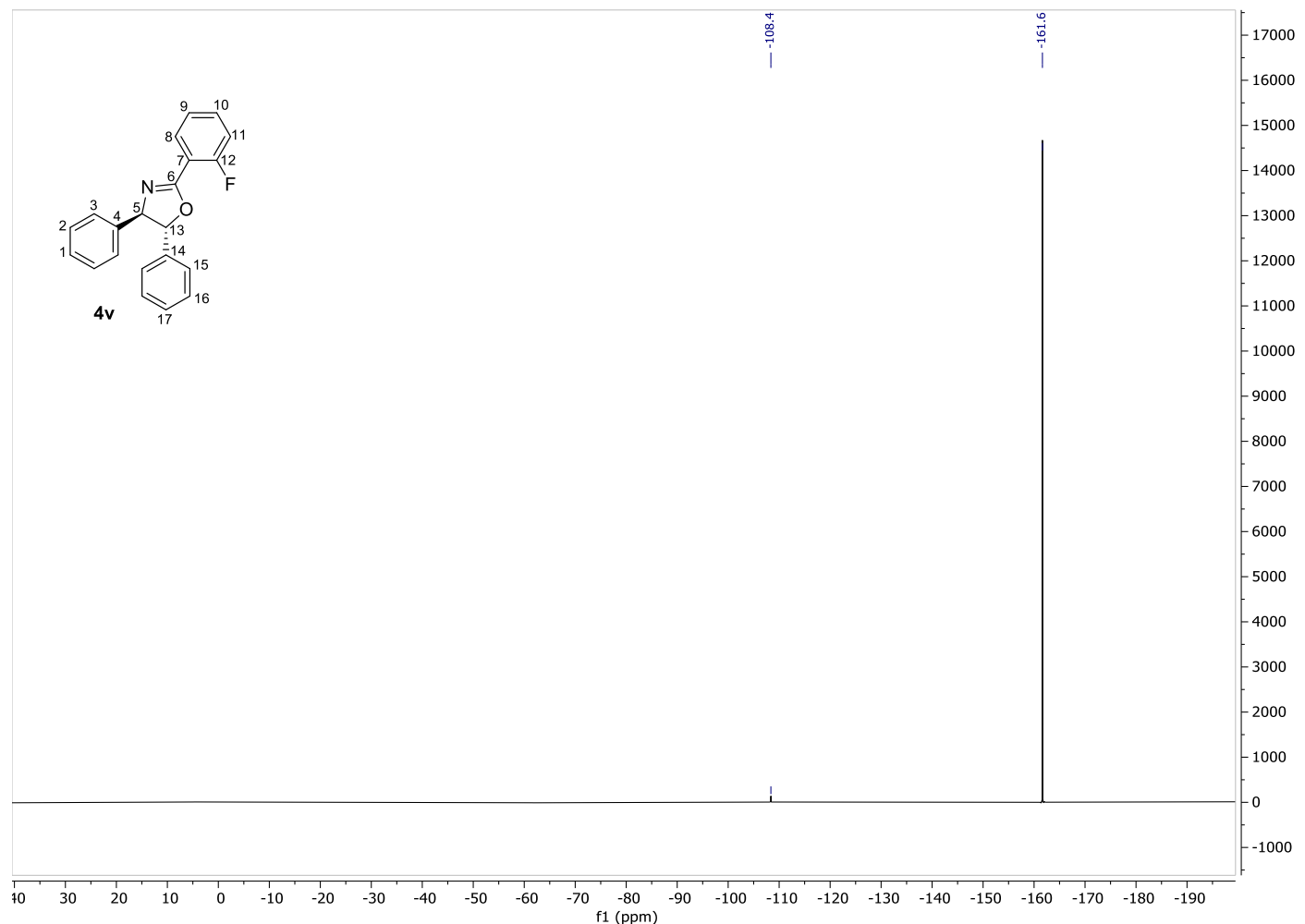




\section{4v-racemic}

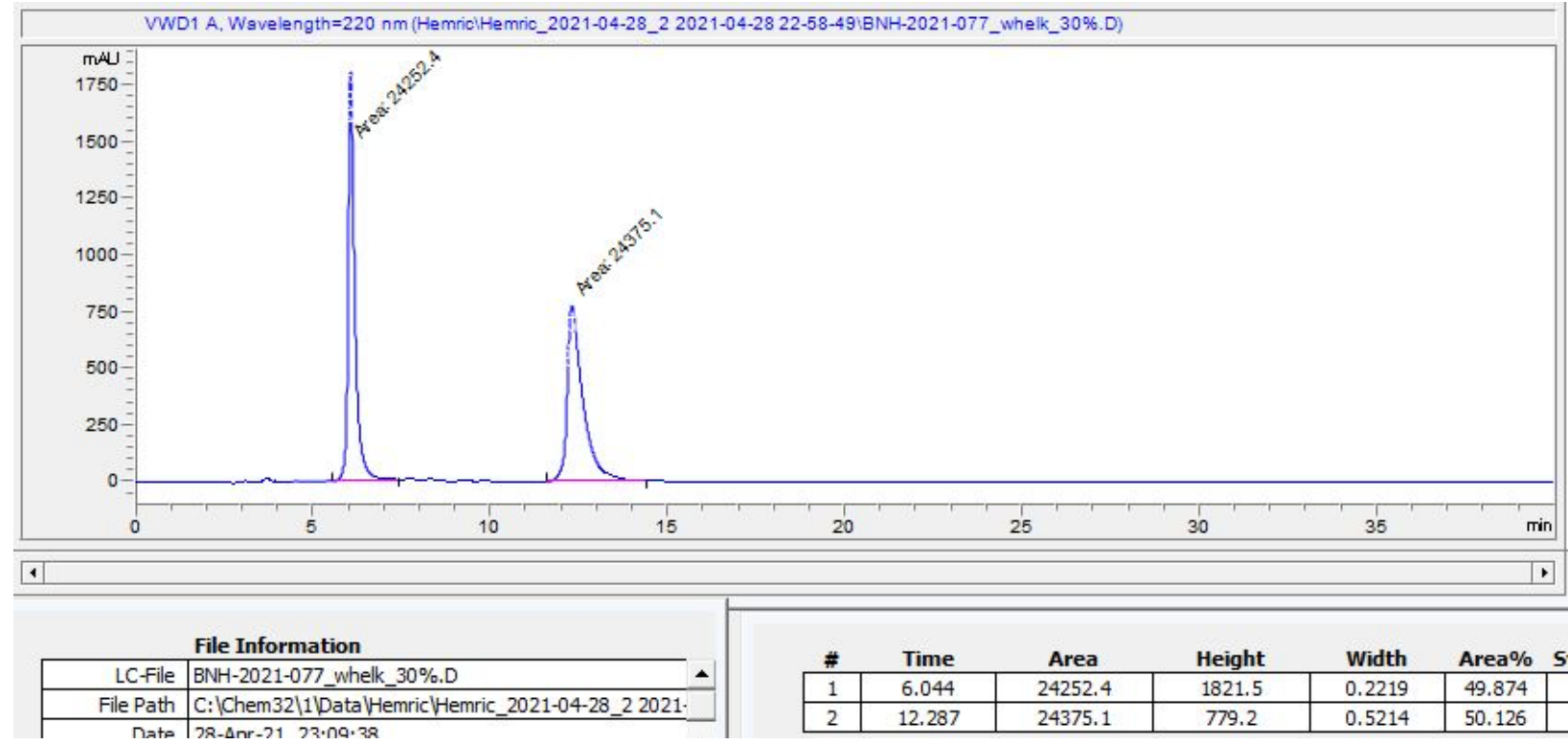

$4 \mathbf{v}-(R, R)$

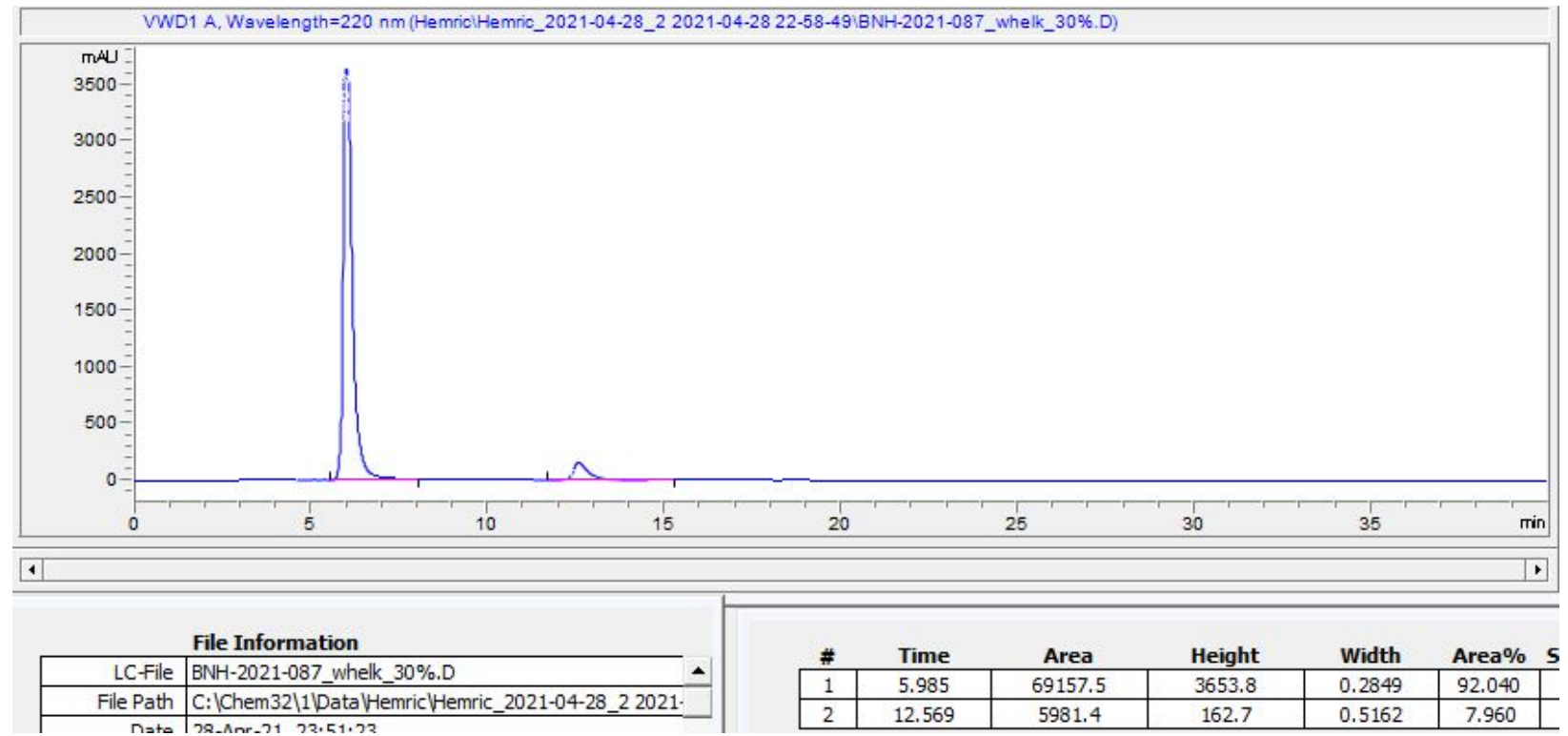


$11{ }^{1} \mathrm{H}$ NMR (500 MHz, $\mathrm{CDCl}_{3}$ )

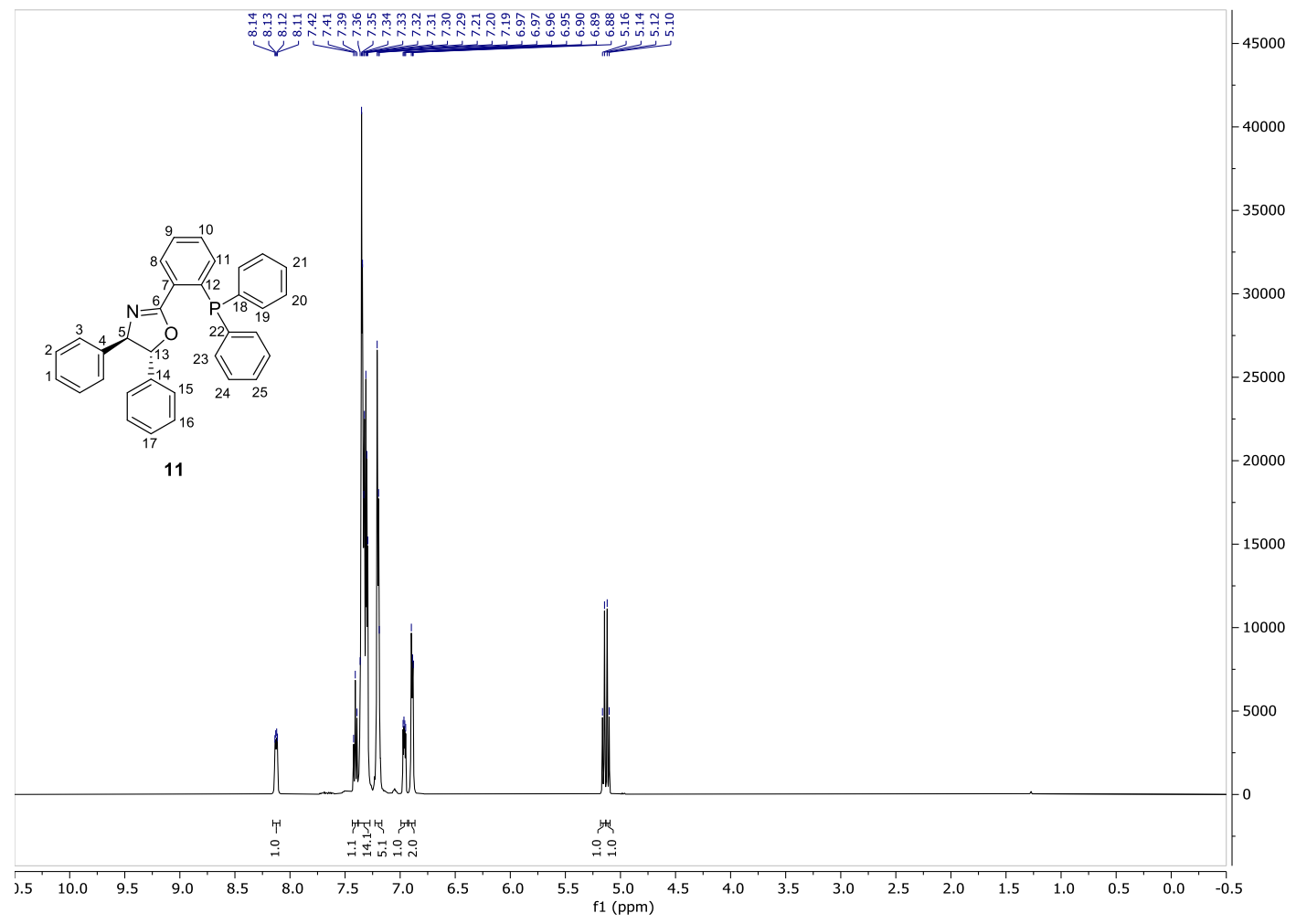

$11{ }^{13} \mathrm{C}$ NMR (126 MHz, $\mathrm{CDCl}_{3}$ )

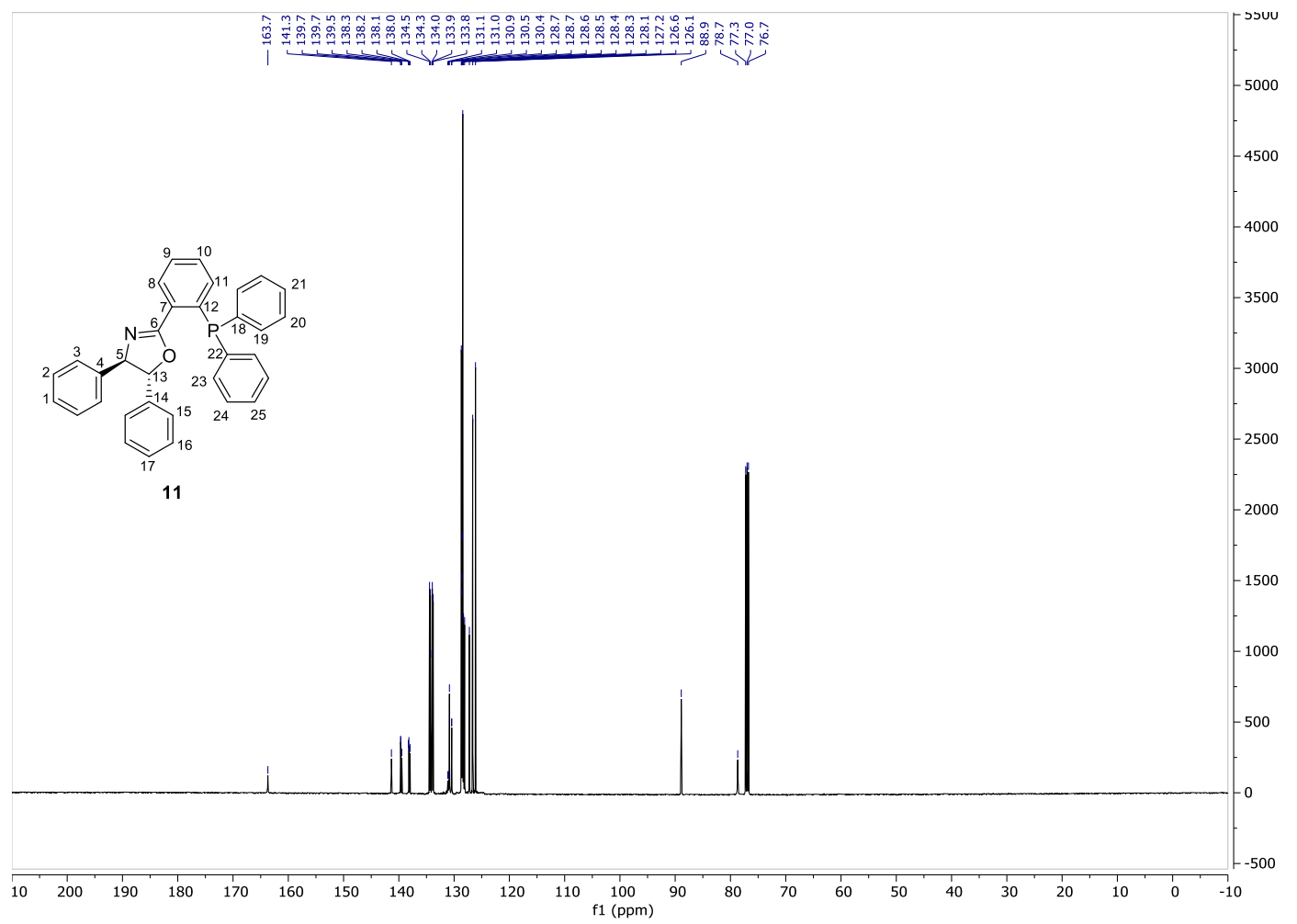


$11{ }^{31} \mathrm{P}$ NMR (202.5 MHz, $\mathrm{CDCl}_{3}$ with $\mathrm{Ph}_{3} \mathrm{PO}$ as standard)

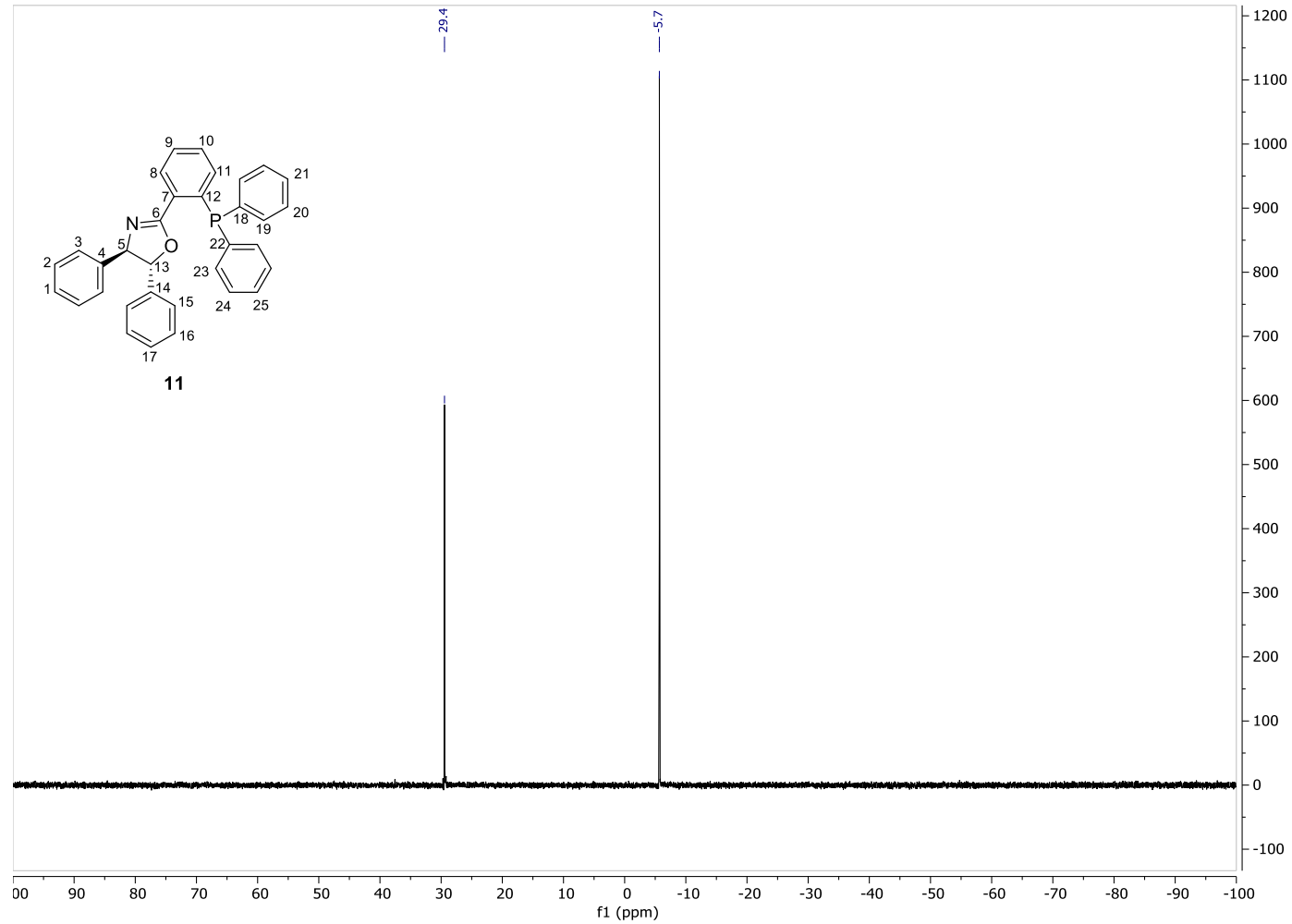

11-racemic

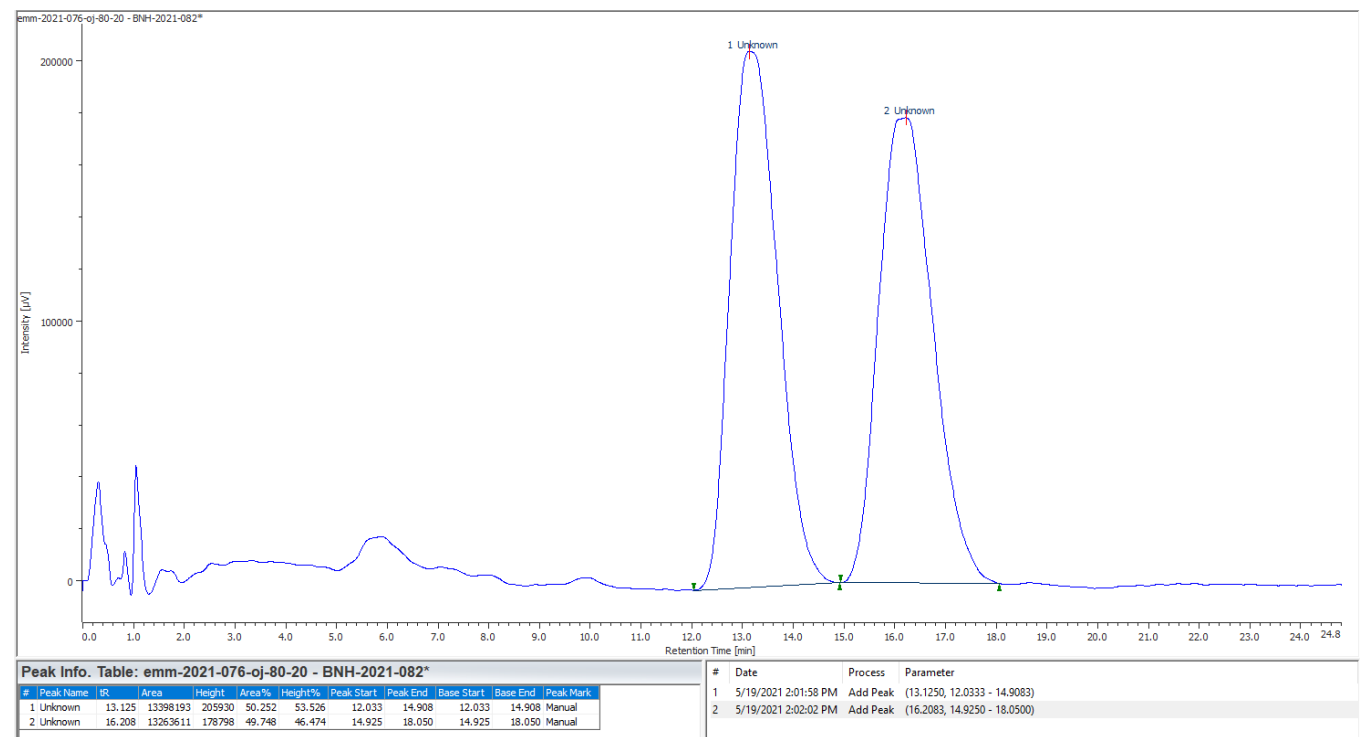


11- $(R, R)-\mathrm{CuI}$ method

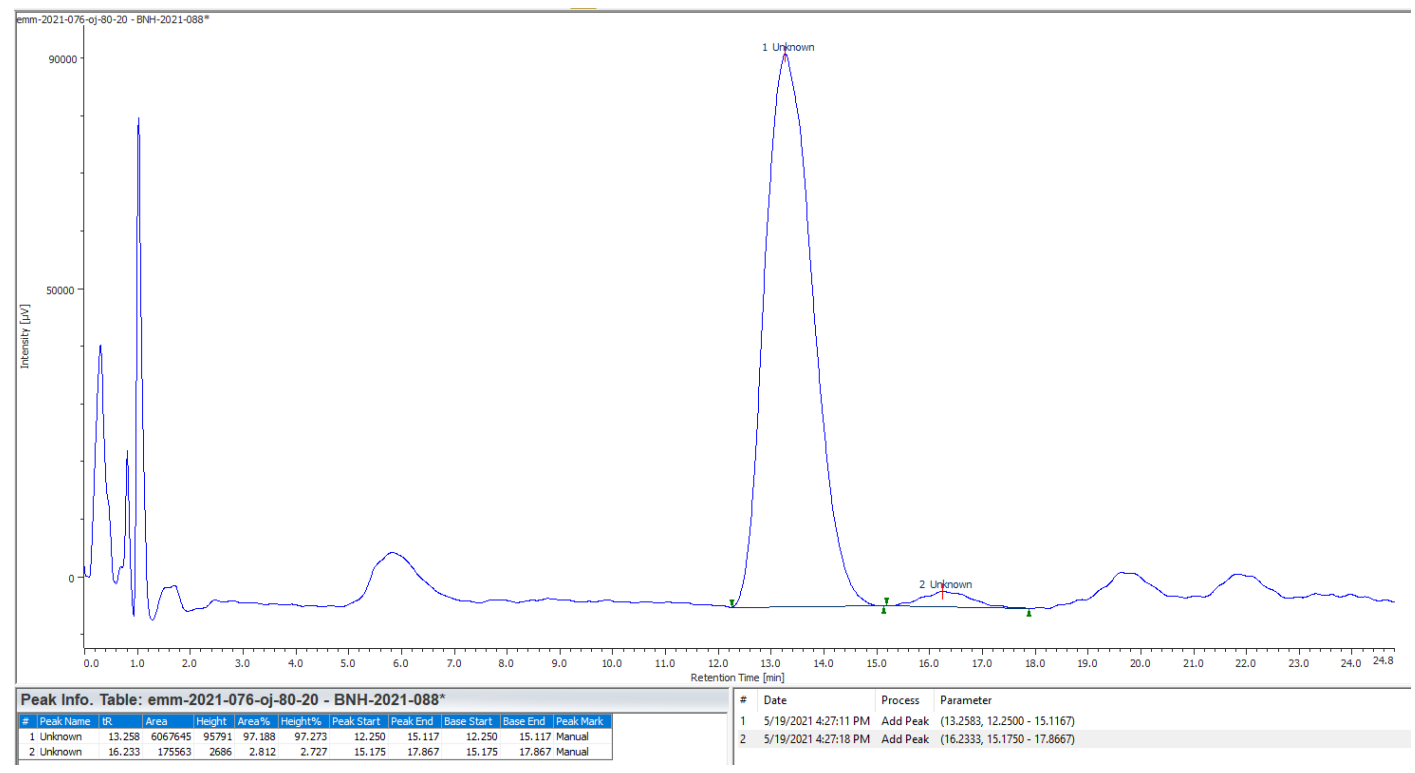

11- $(R, R)-\mathrm{KPPh}_{2}$ method

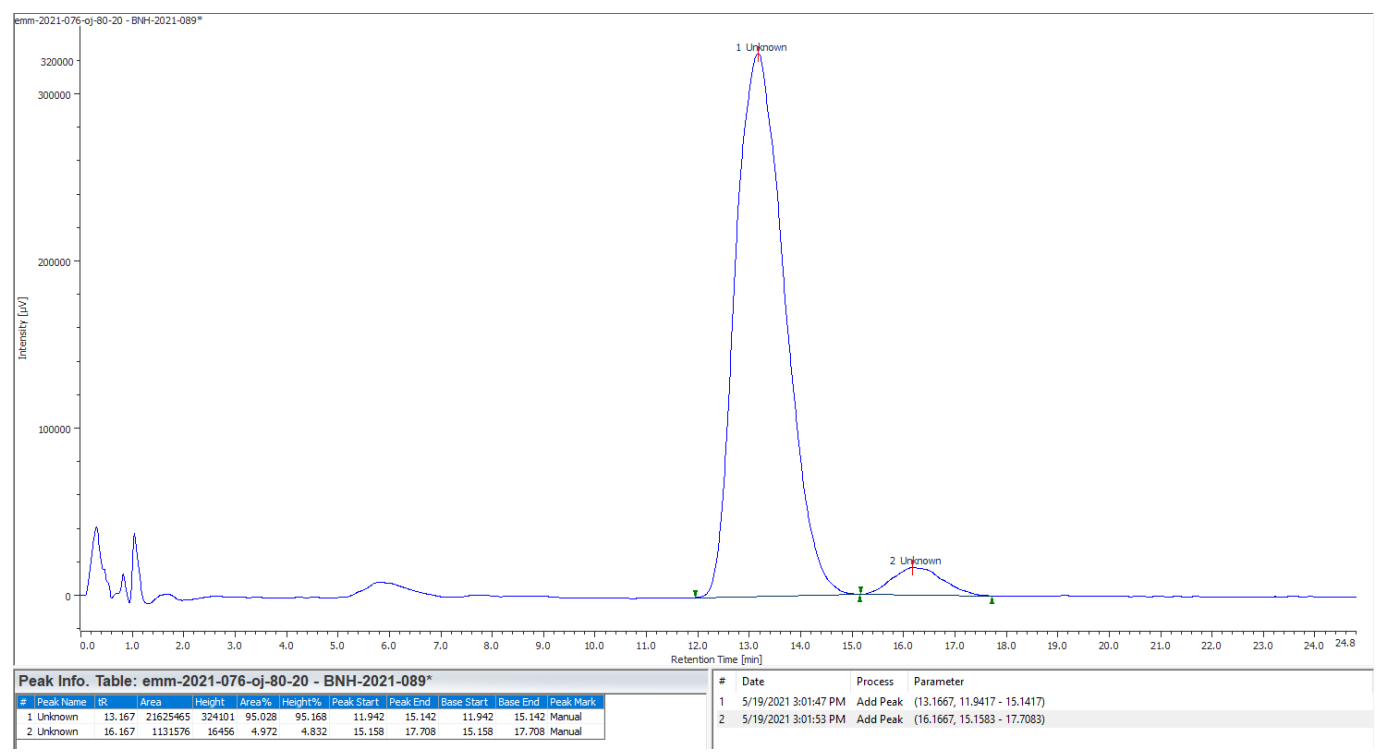

